\title{
FLOW OVER FINITE ISOLATED TOPOGRAPHY
}

by

\section{LuAnne Thompson}

B.S. University of California at Davis

(1983)

A.M. Harvard University

(1986)

Submitted in partial fulfillment of the requirements for the degree of

Doctor of Philosophy

at the

\section{MASSACHUSETTS INSTITUTE OF TECHNOLOGY \\ and the \\ WOODS HOLE OCEANOGRAPHIC INSTITUTION}

November 1990

(c) LuAnne Thompson 1990

The author hereby grants to MIT and to WHOI permission to reproduco.

MARINE BIOLOGICAL LABORATORY

LIBRARY

WOOSS HOLE, MASS.

W. H. O. I. and to distribute copies of this thesis document in whole or in part.

Signature of Author

Joint Program in Physical Oceanography Massachusetts Institute of Technology

Woods Hole Oceanographic Institution

November 26, 1990

Certified by

Accepted by

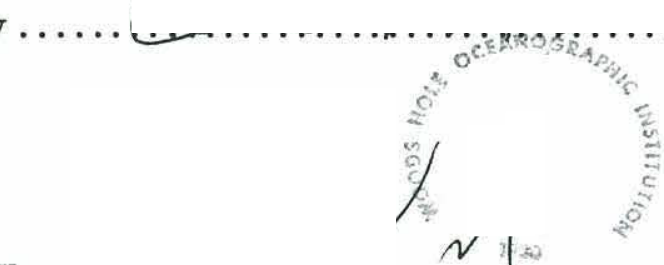

Glenn R. Flierl

Professor

Thesis Supervisor

Carl Wunsch

Chairman, Joint Committee for Physical Oceanography Massachusetts Institute of Technology

Woods Hole Oceanographic Institution 


\begin{tabular}{|c|}
\hline 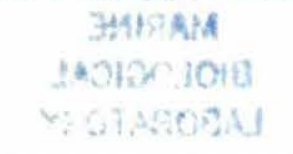 \\
\hline$=3.92511$ \\
\hline 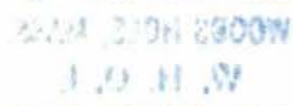 \\
\hline
\end{tabular}




\title{
FLOW OVER FINITE ISOLATED TOPOGRAPHY
}

by

LuAnne Thompson

Submitted in partial fulfillment of the requirements for the degree of Doctor of Philosophy at the Massachusetts Institute of Technology and the Woods Hole Oceanographic Institution

November 26, 1990

\begin{abstract}
One and two layer models are used to study flow over axisymmetric isolated topography. Inviscid or nearly inviscid flow in which non-linear effects have order one importance is considered, and both the effects of $\beta$ and finite topography are included.

A one-layer quasi-geostrophic model is used to find the shape of Taylor columns on both the $\mathrm{f}$-plane and the $\beta$-plane in the inviscid limit of the frictional problem. In this limit, the boundary of the Taylor column is a streamline, and the velocity in both directions vanishes on the boundary. The fluid within the Taylor column is stagnant, corresponding to the solution that Ingersoll (1969) found for flow over a right circular cylinder on the f-plane. In this case, the Taylor column is circular. An iterative boundary integral technique is used to find the solutions for flow over a cone on the f-plane. In this case the Taylor column has a tear drop shape. Solutions are also found for flow on the $\beta$-plane over a cylinder, and the Taylor column is approximately elliptical for westward flow with the major axis in the $x$ direction, while it is slightly elongated in the $y$ direction for eastward flow. The stagnation point of the Taylor column is located on the edge of the topography for all the solutions found. It was not possible to find solutions for smooth topographic shapes.

Steady solutions for flow over a right circular cylinder of finite height are studied when the quasi-geostrophic approximation no longer applies. The solution consists of two parts, one which is similar to the quasi-geostrophic solution and is driven by the potential vorticity anomaly over the topography and the other which is similar to the solution of potential flow around an cylinder and is driven by the matching conditions on the edge of the topography. When the effect of $\beta$ is large, the transport over the topography is enhanced as the streamlines follow lines of constant background potential vorticity. For eastward flow, the Rossby wave drag can be much larger than predicted from quasi-geostrophic theory.
\end{abstract}


A two-layer model over finite topography using the quasi-geostrophic approximation is developed. The topography is a right circular cylinder which goes all of the way through the lower layer and an order Rossby number amount into the upper layer, so that the quasi-geostrophic approximation can be applied consistently. This geometry allows description of flow in which an isopycnal intersects the topography. The model is valid for a different regime than existing models of steady flow over finite topography in a continuously stratified fluid in which the bottom boundary is an isopycnal surface. The solutions contain the two components that are found in the the barotropic model of flow over finite topography. The model breaks down when the interface goes above the topography which occurs more easily when the stratification is weak. Closed streamlines occur more readily over the topography when the stratification is weak, whereas in traditional quasi-geostrophic theory they occur more readily when the stratification is strong. Near the topography, the interface is depressed to the right and raised to the left (looking downstream).

A hierarchy of time-dependent models is used to examine the initial value problem of flow initiation over topography on the f-plane. A modified contour dynamics method is developed that extends the range of problems to which contour dynamics can be applied. The method allows boundary and matching conditions to be applied on a circular boundary. A one-layer quasi-geostrophic model is used to show that more fluid that originates over the topography remains there when the flow is turned on slowly than when it is turned on quickly. Flow over finite topography in a one-layer model shows a variety of different behaviors depending on the topographic height. When the topography has moderate height, two cyclonic eddies are created; when the topography fills up most of the water column, the fluid oscillates on and off the topography as it moves around the topography in a clockwise direction, and none of the fluid is shed downstream. Two quasi-geostrophic stratified models are considered, one in which the topography is small, and the other in which it is finite. In the small topography model, an eddy is shed which is cyclonic, warm-core, and bottom-trapped. In contrast, the shed eddy is cyclonic, cold-core, and surfaceintensified in the finite depth model using the geometry described above.

Thesis Supervisor:

Dr. Glenn R. Flierl, Professor

Massachusetts Institute of Technology 


\section{Acknowledgments}

I have had the good fortune to have worked with many different people during my time in the Joint Program. My advisor, Glenn Flierl, let me work on my own and make my own mistakes. He also taught me persistence. Joe Pedlosky helped me gain confidence in my ability and was generous with his time during Glenn's sabbatical year. Tom Kinder and Harry Bryden introduced me to oceanography were patient with teaching me how to look at data and They also gave me the opportunity to go to sea. Bill Young imparted some of his enthusiasm to me during my first two years when I had the opportunity to have him as an advisor. My advisory committee; Harry Bryden, Nelson Hogg, Joe Pedlosky and Mike McCartney, gave useful advice throughout this work.

I also thank Steve Meacham for being very generous in letting me use his contour dynamics programs and showing me the method that I used in Chapter 2. Dave Chapman was kind enough to discuss early results of his work with a primitive equation model. Mike Spall let me use his laser printer to print out the final copies of my thesis.

My fellow Joint Program students have helped me very much, most recently editing much of this thesis. Elise Ralph generously let me use her computer during the last hectic months of finishing the thesis. Last but not least I want to thank Greg Johnson for giving me all the support that I needed over the last five years.

This study was supported by the Office of Naval Research Program N0001490-J-1839. 


\section{Contents}

$\begin{array}{ll}\text { Abstract } & 3\end{array}$

$\begin{array}{ll}\text { Acknowledgments } & \mathbf{5}\end{array}$

1 Introduction $\quad 9$

1.1 Observations of flow over isolated topography in the ocean . . . . 11

1.2 Review of modeling results . . . . . . . . . . . . . . 14

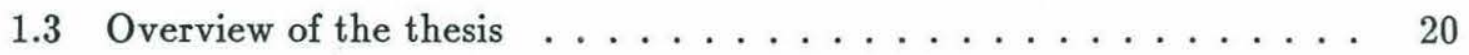

2 Frictional Taylor columns on the $\beta$-plane 25

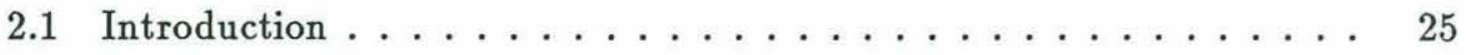

2.2 Model formulation $\ldots \ldots \ldots \ldots \ldots \ldots \ldots \ldots \ldots$

2.3 Numerical technique $\ldots \ldots \ldots \ldots \ldots$

2.4 Taylor columns on the $f-$ plane $\ldots \ldots \ldots \ldots \ldots$

2.5 Taylor columns in a westward flowing current on the $\beta$-plane . . . . 44

2.6 Taylor columns in an eastward flowing current . . . . . . . . 45

2.7 Summary and conclusions $\ldots \ldots \ldots \ldots \ldots \ldots$

3 Steady solutions for flow over finite topography 62

3.1 Introduction . . . . . . . . . . . . . . . 62 
3.2 Background ........................... 63

3.3 Flow over finite topography in one layer . . . . . . . . . 66

3.3.1 Model formulation . . . . . . . . . . . . . . 67

3.3.2 Calculation of quantities derived from $\psi \ldots \ldots 71$

3.3.3 Solutions on the $\mathrm{f}$-plane ............... 74

3.3.4 Solutions for westward flow on the $\beta$-plane . . . . . . . . 75

3.3.5 Solutions for eastward flow on the $\beta$-plane . . . . . . . . 88

3.3.6 Summary: barotropic flow over finite topography . . . . . 99

3.4 Flow over finite topography in a two-layer model . . . . . . . . 102

3.4.1 Model formulation ................. 106

3.4.2 Barotropic flow on the f-plane . . . . . . . . . . . 111

3.4.3 Flow on the f-plane with vertical shear . . . . . . . . . 123

3.4.4 Westward flow on the $\beta$-plane . . . . . . . . . . 125

3.4.5 Eastward flow on the $\beta$-plane . . . . . . . . . . 129

3.4.6 Summary: flow over finite topography in a two-layer model . . 131

4 Time dependent solutions $\quad 135$

4.1 Introduction and background ................ 135

4.2 Flow in one layer over small topography . . . . . . . . . . . 138

4.2 .1 Numerical method . . . . . . . . . . . . 139

4.2 .2 Numerical solutions . . . . . . . . . . . . . . . 143

4.3 Flow in one layer over finite topography . . . . . . . . . 153

4.3.1 Numerical method ................. 156

4.3 .2 Numerical solutions . . . . . . . . . . . . . . 161

4.4 Flow in two layers over small topography . . . . . . . . . . . 169 
4.4.1 Numerical Method ... . . . . . . . . . . . . 170

4.4 .2 Numerical solutions . . . . . . . . . . . . . . . . . 172

4.5 Flow over finite topography in two layers . . . . . . . . . . . . 177

4.5.1 Numerical Method ... . . . . . . . . . . . 181

4.5.2 Numerical solutions . . . . . . . . . . . . . . . . . . . 184

4.5.3 Summary: Flow over topography in a stratified model . . . . . 192

4.6 Summary and conclusions . . . . . . . . . . . . 196

5 Conclusions

199

5.1 A Summary of the thesis . . . . . . . . . . . . . . . 199

5.2 Advantages and limitations of the models . . . . . . . . . . . 205

5.3 Relevance of modeling results to the ocean . . . . . . . . . . . 207

5.4 Future work . . . . . . . . . . . . . . . . 209

$\begin{array}{ll}\text { References } & \mathbf{2 1 0}\end{array}$

$\begin{array}{lr}\text { Appendix A } & 214\end{array}$

$\begin{array}{lr}\text { Appendix B } & 216\end{array}$

$\begin{array}{lr}\text { Appendix C } & 218\end{array}$ 


\section{Chapter 1}

\section{Introduction}

The influence of bottom topography on oceanic flow is widespread. It can alter the path of large scale currents such as the Gulf Stream or deep western boundary currents and can play an important role in the vertical distribution of momentum in places such as the Antarctic circumpolar current and possibly in the large scale circulation elsewhere. Isolated topography can greatly affect the flow pattern at the synoptic scale. It can cause the generation of eddies, deflection of the flow field and the creation of pockets of trapped fluid such as Taylor columns. These phenomena can influence large scale flow and small scale features in both the dynamical patterns of the current and density fields and the structure of passive fields, such as distribution of flora and fauna. Topographic features can also affect the energy balance for large scale currents by exerting drag on the flow. There are additional features which are exhibited at shorter time and space scales than synoptic, including topographic rectification of tidal motions, internal wave trains, enhanced boundary mixing, and the generation of trapped topographic waves.

Isolated topography can thus influence ocean circulation over a wide range of time and space scales. It is important to understand its effect since much of our oceanographic intuition has been derived from the study of large and small scale motions in the absence of topographic variations. One would like to be able to 
parameterize the effect of the many topographic features in the ocean in larger circulation models. The ocean bathymetry is made up of ridges, isolated seamounts and seamount chains and fracture zones, and these features should all be understood separately before an understanding of their effect on the general circulation can be included in existing large scale models.

Although numerous papers have been written on the subject of flow over isolated topography in a rotating fluid, there are still gaps in our understanding of the interaction between flow and topography as applicable to the ocean. Taylor (1917) discovered that fluid which is homogeneous and rapidly rotating has flow that is nearly independent of the coordinate parallel to the axis of rotation. He observed in the laboratory that when there is a bump in a rapidly rotating fluid with uniform density, the flow tends to go around instead of over the topographic feature, leaving an undisturbed column over the topography (Taylor, 1923). This phenomenon was first called a Taylor column by Hide (1961). In a stratified fluid, a similar thing happens, although the Taylor column tends to be bottom trapped and has a depth dependent structure. Oceanographers have studied flow near topography to discover if Taylor columns can be observed in the ocean.

A brief discussion of flow over isolated topography in the atmosphere is useful for understanding how modeling of oceanic flow over isolated topography requires a different approach than the modeling of such flow in the atmosphere. The size of topographic variations are approximately the same in the ocean and the atmosphere, but the ocean is more weakly stratified than the atmosphere, and the vertical penetration scale relative to the total depth in the ocean is larger than in the atmosphere. As a result Taylor columns (or caps) are more likely to be observed in the ocean than in the atmosphere. The radius of deformation in the ocean is smaller hence synoptic scale motions interact with smaller topographic scales. In the ocean relative vorticity is smaller and potential vorticity anomalies $f_{0} h_{0} / H$ (where $f_{0}$ is the Coriolis 
parameter, $h_{0}$ is the height of the topography, and $H$ is the total depth) are relatively more important. In contrast to the atmosphere, isolated topographic features can take up a larger fraction of the water column in the ocean since seamounts often reach close to the surface, requiring a different approach in the modeling of flow over finite topography than has been taken in the atmosphere (for more of a discussion of the role of topography in the atmosphere and the ocean see Holloway and Muller, 1990). The modeling in this thesis has particular relevance to the ocean because it concentrates both on the Taylor column problem in a barotropic fluid and on order one topography in both a one and a two-layer model.

Because of the wide range of both time and space scales that isolated topography can influence and because of variability in the background flow field, dynamical interpretation of oceanic observations of the interaction of flow and isolated topography is difficult. In this thesis, we model phenomena that occur on the synoptic scale, so we concentrate on observations relevant to this scale of motion. Studies of the effect on flow as it passes isolated topography are limited to a few experiments.

Below we discuss some observations of flow over isolated topographic features in the ocean, concentrating on studies of flow over seamounts. Then we review some of the modeling that has been done on flow over isolated topography. Finally, we give a brief overview of the thesis.

\subsection{Observations of flow over isolated topography in the ocean}

Some of the first evidence of the local effect on mesoscale flow over a seamount was given by Vastano and Warren (1976). In this study they observed flow over the Atlantis II seamount, which rises to $1645 \mathrm{~m}$ from the surface of the ocean, over $3000 \mathrm{~m}$ 
from the bottom. With STD stations they produced maps of potential temperature at different depths. The Gulf Stream was not stationary during their study, so that the quasi-synoptic pictures were difficult to interpret. However, they were able to observe a warm-core eddy over a cold-core eddy in the lee of the seamount. In addition, below the top of the seamount, the lines of constant potential temperature (which we interpret loosely as streamlines) split to go around the seamount. It was not clear if there were closed streamlines over the topography because of the large spacing between stations and the time-variability in the region.

The interpretation of these observations in terms of well understood quasigeostrophic dynamics is difficult partly because the seamount that Vastano and Warren (1976) studied was so tall. The Abyssal Topography Experiment was designed to study flow over small topography where the dynamics were expected to be simpler. Over a small isolated hill (500 m high) on an abyssal plain, isotherms tended to be raised and the effects of the hill penetrated about 2.5 times its height into the water column (Gould et al, 1981). Current meter, float, and CTD observations were used to describe the flow over the hill. In addition to the raising of the isotherms, the flow velocity was enhanced over the left hand side (looking downstream) of the topographic feature, qualitatively consistent with quasi-geostrophic theory. However, the rise of the isotherms was not as high as would be expected from quasi-geostrophic theory, indicating that the finite height of the topography (although it was only 0.1 times the total depth of the water) caused the flow to go around the topography at the lowest levels. In this experiment it was shown that even topography as small as this $500 \mathrm{~m}$ hill can have a profound influence on the flow field.

Observations of the flow structure over a seamount in the recirculation system of the Gulf Stream indicated that, as quasi-geostrophic theory predicts, there was anticyclonic motion over the seamount and a bottom trapped perturbation that extended far up into the water column (Owens and Hogg, 1980). Measurements of 
both density and direct current allowed the calculation of relative vorticity above the seamount; it was negative and had the approximate magnitude predicted from a quasi-geostrophic theory. The seamount studied was fairly small, rising only $400 \mathrm{~m}$ from the sea floor.

Two freely drifting buoys that were originally in the Gulf Stream were observed to loop over of the Corner Rise seamounts and continue looping in their lee (Richardson, 1980). These seamounts rise up within $650 \mathrm{~m}$ of the sea surface, indicating that quasi-geostrophic theory is probably not relevant for the description of the flow field near them. The buoys were trapped within a warm-core anticyclonic eddy which was then shed downstream. The sense of the circulation is consistent with theoretically predicted circulation for what would appear over the seamount, but inconsistent with the sense of circulation of shed eddies in an inviscid theory. Instead, the shed eddies could have originated over the topography, and been subsequently shed downstream through frictional effects.

The Kuroshio crosses the Emperor Seamount Chain which reaches up within $500 \mathrm{~m}$ of the surface in places. Northward deflection (anticyclonic looping) of the current north of the seamounts has been observed (Roden and Taft, 1985). Spreading of the isotherms indicated that the current weakened over the topography. The position of the pycnocline uplift varied from seamount to seamount. A bottom-trapped Taylor-column-like feature was observed over the shortest seamount, but, over the other seamounts, current meanders or shed eddies were observed. The interpretation of the complicated density field seen in this experiment was not possible using simple models and indicated how much we still have to understand about oceanic flow over topography. A further review of flow over isolated seamounts including motions with smaller time and space scales is given by Roden (1987).

It should be clear from this review of some of the observations of flow over topography that there are several features that are not well understood. First, 
seamounts are often quite tall, so study of flow over finite topography is desirable. Second, the response tends to be bottom trapped, or at least to appear in only part of the water column (c.f. Vastano and Warren, 1976). This suggests the importance of considering flow in stratified models. The complicated nature of the background flow for any real oceanic situation makes the detailed modeling of the flow interaction difficult and time-dependent modeling necessary. In this thesis, simple flow situations are studied to try to isolate the important dynamics that could be at work in the ocean by including each of the effects mentioned above: finite topography, stratification, and time-dependence.

\subsection{Review of modeling results}

The structure of Taylor Columns and more detailed interaction between flow and topography have been extensively modelled since their initial discovery. With few exceptions, the models have been limited to flows with small Rossby numbers and small topography. Thus most of the models are quasi-geostrophic, which we suggest above may be inadequate for discussing many important features of flow over finite topography in the ocean. Past work has included the study of flow in homogeneous fluid, layered fluid, and continuously stratified fluid.

Initially, the approach to the study of flow over isolated topography was to examine it in the laboratory beginning with Taylor's (1923) seminal work. Early laboratory models (Hide and Ibbetson, 1966, and Hide et. al. 1968) confirm Hide's (1961) conjecture that the obstacle height must be greater than $\alpha h_{0} / \epsilon$ for a Taylor column to form, where $\alpha$ is some order one number, $h_{0}$ is the height of the topography relative to the total depth of the fluid and $\epsilon$ is the Rossby number. In the laboratory the flow shows a pronounced left-right asymmetry looking downstream. These initial studies were confined to f-plane homogeneous flows. 
To make analytical progress in the modeling of the stagnant Taylor column feature, Jacobs (1964) ignored inertial effects and considered the flow in the presence of Laplacian friction. When the flow is linear and viscous, the viscous effects are confined to Ekman boundary layers on solid surfaces and to a free shear-layer coincident with the vertical cylinder circumscribing the bottom obstacle. The flow above the cylinder is stagnant and outside the cylinder the flow is irrotational. Under this analysis, it is assumed that the Rossby number is small while $h_{0} / \epsilon$ is large. The Taylor column covers nearly all of the obstacle. The resulting flow field lacks the left-right asymmetry seen in the laboratory, and is symmetric upstream-downstream. The aspect ratio of the fluid is order one in Jacobs' (1964) model, corresponding to the laboratory situation. In the ocean, the aspect ratio is generally small, even for flow over very tall seamounts (i.e. $5 \mathrm{~km}$ vertical height verses $50 \mathrm{~km}$ horizontal width). In addition, non-linear terms are probably important, as can be seen by the asymmetry in the flow field in the laboratory. Thus, the validity of this solution for oceanographic situations is probably quite limited.

The effects of non-linearities without the influence of friction have been studied extensively. Clearly, some combination of frictional and inertial effects is needed to thoroughly understand the phenomenon of flow over topography in the ocean, but it is difficult mathematically to handle the general problem. The inviscid solution shows the same left-right asymmetry as is seen in the laboratory, although in an inviscid model it is difficult to understand how the Taylor column could become stagnant. The problem was initially considered in the quasi-geostrophic framework.

The inviscid problem has been solved in general by letting the potential vorticitystream function relationship be determined from upstream conditions. Once streamlines close, fluid is trapped and a Taylor column should form; at this point, the potential vorticity-stream function relationship derived from upstream is questionable within the trapped region. Within closed streamlines, the flow is not stagnant 
and has anticyclonic circulation. These solutions could be correct over a time scale which is much less than the spin up time of the fluid. Hogg (1973) found the solution for flow over a right circular cylinder in a continuously stratified fluid including the effect of background shear. The Taylor cap is conical and decays with distance above the seamount. The effect of $\beta$ was considered by McCartney (1975) in a two-layer model. He found that for westward flow on the $\beta$-plane the flow looks qualitatively similar to that on the f-plane. For eastward flow however, stationary Rossby waves exist downstream of the topography. There have been several studies of flow which include both the effects of continuous stratification and $\beta$ (Janowitz, 1975; Johnson, 1977 and Johnson, 1979). All of these models have used the quasi-geostrophic approximation.

In each model, closed streamlines form once the topography is above a certain height as predicted by Hide (1961). These closed streamlines must spin down ultimately giving a frictional solution over time scales comparable to the spin up time. Huppert (1975) considered the problem of finding the critical height for which closed streamlines form for flow on the f-plane for a general axisymmetric obstacle including the effect of stratification. He found that for barotropic flow, the obstacle shape has little effect on the results with a small change in the parameter $\alpha$. For stratified flow, if the obstacle has any vertical faces, a Taylor column forms no matter how small the bump is.

In the inviscid solutions, fluid can be trapped within closed streamlines which occur outside of the support of the topography. When closed streamlines occur, fluid that is trapped within the closed streamlines originates upstream. This is not consistent with what occurs in the laboratory. There have been several approaches for determining the solution for the Taylor column shape once closed streamlines have formed. Ingersoll (1969) provided a way to close the problem by allowing a different potential vorticity-stream function relationship within closed streamlines; the fluid is 
stagnant and the stream function is constant. The flow within closed streamlines is found by considering how bottom drag would act on the fluid in the limit of vanishing viscosity. He found analytic solutions under special circumstances. It is not clear how this requirement generalizes to models which contain stratification.

Another approach was given by Johnson (1983) who interpreted the Taylor column as a region of fluid which is retained over the topography after the flow is turned on in an initial value problem. He looked for solutions in which the maximum amount of fluid is retained over the topography. He surmised that this solution is set up over the advection time scale. It is identical to an Ingersoll type frictional solution for uniform flow and flow with negative linear shear. When the background shear is positive, the two forms of solution differ. The boundary of the Taylor column for the maximum retention solution is an ellipse which is contained within closed streamlines. When the effect of the shear is large, Johnson finds the Ingersoll type solution. In this solution, the Taylor column has finite extent in $y$ and is infinitely long in the $x$ direction. The Ingersoll type frictional solution is set up over a spin-up time and would ultimately be the steady state solution.

The more oceanographically realistic situation of topography of finite height has also been considered in the inviscid model, although the study has been limited primarily to situations in which the bottom boundary remains an isopycnal surface. For the ocean this approximation is questionable because of the large fractional height (the height of the topography relative to the total depth of the fluid) of many topographic features. Merkine and Kalnay-Rivas (1976) studied the problem using the geostrophic momentum approximation. They found that near the topography the flow is baroclinic, while far away it remains barotropic, and there is a trapped vortex over the topography which has flow which is independent of the incoming velocity once the flow has been set up. This is just a consequence of conservation of potential vorticity in the fluid that originated upstream and ends up over the topography. The 
flow field is a combination of the bound vortex and the background flow field with the perturbation pressure field. In quasi-geostrophic solutions the flow field is simply a linear combination of the bound vortex solution and the background flow field. Once again, when closed streamlines form the potential vorticity-stream function relationship in the trapped region is determined from upstream conditions, and the solution is valid over a time scale less than the spin up time.

Buzzi and Speranza (1979) defined two classes of quasi-geostrophic solution and found steady solutions for stratified flow with two obstacle shapes. First they considered flow over a hemispherical obstacle. For this geometry, they required that the bottom surface has constant specific entropy, or potential temperature, as did Merkine and Kalnay-Rivas (1976). The solution looks similar to other quasi-geostrophic solutions over similar obstacles. They then considered flow past a half disk standing vertically in the fluid. In this case, the streamfunction at the wall depends only on the vertical coordinate and is specified arbitrarily. They chose to require that the stratification is constant on the wall. Since the flow cannot penetrate through the wall at the lowest level, the flow has to go around the obstacle, defining a different class of quasi-geostrophic solutions. One might expect that in a general stratified model with flow over topography of finite vertical and horizontal cross sections, the solutions would contain elements of the two types of solutions that they discussed.

Schar and Davies (1988) further explored this problem by considering the solution in isopycnal coordinates. They required the bottom to be an isopycnal surface. The assumptions of the model break down when the stratification is too strong or the height of the topography is too large such that isopycnals intersect the topography. Likewise, closed streamlines form when the stratification becomes strong or the topography becomes large.

Steady solutions give useful insight into important dynamical processes, but they are inadequate for two reasons. We have seen above that the oceanic flow is 
highly variable, so it is unlikely that steady state solutions could be maintained over long periods of time. Also, we would like to understand when the steady solutions that have been found are the final state of an appropriate initial value problem.

The initial value problem has been examined for flow over relatively small topography. The first such study was the most ambitious. Huppert and Bryan (1976) solved the initial value problem of flow over topography in a periodic domain using a primitive equation model with continuous stratification. Because the stratifcation is strong, the flow in the lowest levels goes around instead of over the topography. The topography that they used is fairly small, comparable to the topography studied in Gould et. al (1981). In their study they demonstrated two different flow regimes, one in which the fluid which originated over the topography is trapped there, and one in which it escapes downstream. The fluid which escapes is contained within a cyclonic eddy. They also developed a simple point vortex model to help describe some of the shed eddy's behavior. James (1980) realized that much of the dynamics that Huppert and Bryan (1976) found could be explained in a much simpler quasigeostrophic context. He considered the time-dependent forces on the topography in a barotropic quasi-geostrophic model.

Verron and LeProvost (1985) studied the initial value problem on the $\beta$-plane. Rossby waves are generated downstream of the topography for eastward flow. These solutions were limited somewhat because the model is confined to a channel in $y$ (the north-south direction) and is periodic in $x$ (the east-west direction). Their model runs never reached steady state. Finally, Verron (1986) considered oscillating flow and reproduced anticyclonic shed eddies due to the effect of bottom drag, similar to those observed and reported by Richardson (1980). 


\subsection{Overview of the thesis}

The modeling of flow over seamounts in the ocean is difficult because there is a wide range of space and time scales that need to be understood in order to fully explain what is seen in the ocean. We choose to ignore effects of the flow that occur on scales other than synoptic and concentrate instead on simple models that may have qualitative relevance to flow in the ocean. We extend the work that has been done on the modeling of flow over topography by developing models which include both the effects of $\beta$ and finite topography, and a simple model which allows the intersection of an isopycnal with the topography. We consider both steady solutions and the initial value problem. We use simple models that allow analytic or semi-analytic solutions. The model geometries are introduced in Figure 1.1.

We look at solutions to flow over topography on three different time scales. We extend Ingersoll's (1969) work and study the steady-state solutions on the $\beta$-plane to find Taylor column solutions that are valid for time scales comparable to the spin up time. We then extend the work done on steady inviscid solutions. When closed streamlines form, these solutions are valid for time scales short compared to the spin up time. Finally, we study solutions to the initial value problem and model the flow in the first few advection times. Throughout the thesis we concentrate on flow over tall topography in which we expect there to be fluid trapped near the topography. Flow over small bumps is studied to compare and contrast to the finite topography work. The structure of the region of trapped fluid is different in each model as is described below.

In Chapter 2 we use Ingersoll's (1969) conditions on the flow at the boundary of the Taylor column and a boundary integral technique to find solutions when closed streamlines have formed and the action of bottom drag causes the fluid within the closed streamlines to be stagnant. The model geometry that we use in Chapter 2 


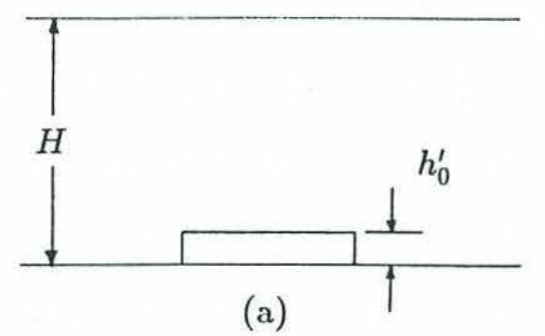

(a)

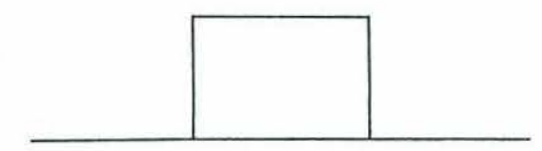

(b)

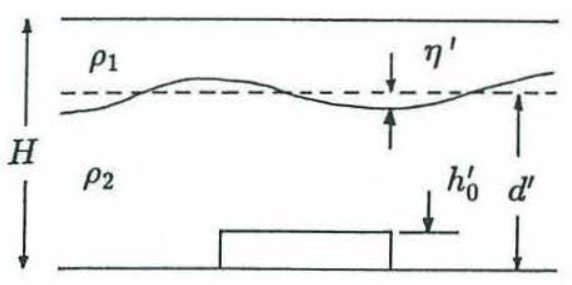

(c)

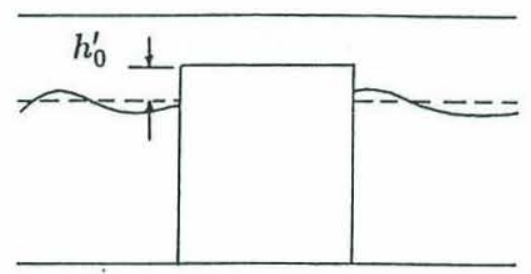

(d)

Figure 1.1: Side view of the four models that are considered in this chapter. In each case there is uniform barotropic incoming flow of size $U$ and each has a rigid lid. The topography has radius $L$. a) One layer model with quasi-geostrophic topography. The topography has height that is order Rossby number with respect to the total layer depth. b) One layer model with finite topography. Both the topography and the Rossby number are finite. c) Two layer model with small topography. The lower layer depth is $d^{\prime}$ while the density of the upper and lower layers is $\rho_{1}$ and $\rho_{2}$ respectively. d) Two layer model with finite topography. The topography extends an order Rossby number amount into the upper layer and the interface intersects the topography. The prime quantities are dimensional. 
is shown schematically in Figure 1.1a. We extend Ingersoll's (1969) solutions to flow on the $\beta$-plane and over a right circular cone on the $\mathrm{f}$-plane, allowing further understanding of Taylor columns in a barotropic quasi-geostrophic fluid. In these solutions, the region of trapped fluid is stagnant and fluid is only trapped within a region which is contained within the support of the topography.

The solution for westward far field flow consists of a Taylor column on the northern side of the topography. As the horizontal extent of the topography is increased and the effect of $\beta$ becomes more important, the Taylor column becomes more elliptical with the major axis in the east-west direction. When the far field flow is eastward, stationary Rossby waves are generated downstream of the topography. The size of the Taylor column is then only weakly dependent on the horizontal extent of the topography, but its location changes as the Rossby wave pattern changes downstream. Additional closed streamlines appear downstream of the topography when the effect of $\beta$ is large. These closed streamlines do not contain stagnant fluid; thus the solution is no longer consistent with Ingersoll's formulation.

In Chapter 3, we allow the topography to be finite and quasi-geostrophic theory is no longer valid as shown in Figure 1.1b. This model allows both the effects of $\beta$ and finite Rossby number and also allows horizontal divergence of fluid around the obstacle. Because we study flow over the right circular cylinder, an analytic solution is possible. The stream function consists of two parts, one which is odd in $y$ and is similar to the solution of flow around an island, and one which is even in $y$ and is similar to the quasi-geostrophic solution of flow over topography. As in the inviscid models that we reviewed earlier, when closed streamlines form these solutions are valid for a time scale short compared to the spin-up time. From this model, we can see that there can be larger effects of the topography on the background flow field than predicted from quasi-geostrophic theory. This is shown by calculating the lift and drag on the obstacle, as well as the circulation induced over the obstacle. 
When the flow is eastward, a set of lee wave functions is constructed that satisfies the correct upstream boundary conditions, and this set is used to find the solution. When the $\beta$-effect is strong, the largest difference between the quasi-geostrophic and finite depth solutions is seen. In this regime, there is enhanced transport over the topography for westward flow, and the wave drag for eastward flow is much larger than that predicted by quasi-geostrophic theory.

Since the effect of stratification is important in the ocean, we develop an inviscid model that shows effects resulting from the combination of finite topography and stratification. This work extends the work of Buzzi and Speranza (1979). We use quasi-geostrophic theory to describe the interaction of flow with an obstacle that has finite cross section in the vertical and the horizontal. We consider a two-layer model in which the topography penetrates all of the way through the lower layer and an order Rossby number amount into the upper layer as shown in Figure 1.1d. The topography is a right circular cylinder, and an analytic solution is found using the same mathematical techniques we use in the finite depth one-layer solution. In this way, the flow around a non-quasi-geostrophic topographic feature is described using quasi-geostrophic theory. As in the barotropic solution, the stream function consists of two parts with differing symmetries. The breakdown of the model occurs when the interface rises above the topography, or when closed streamlines occur so that the inviscid solution becomes suspect over long time scales.

Once the steady state solutions are understood, the initial value problem can be examined. We only consider the first few advective time scales to look particularly at the shedding of fluid off of the topography. We construct a hierarchy of models that allows the simulation of the set up process in a variety of situations. The relevance of the inviscid steady state solutions is explored as well as the dynamics that are important when time-dependence is included. We choose to use a modified contour dynamics model which requires that our numerical experiments be done on the f- 
plane. This allows us to isolate how stratification and finite topography affect the eddy shedding process without resorting to simulations with a primitive equation model.

We begin by studying a one-layer quasi-geostrophic model (Figure 1.1a) which captures much of the important dynamics in the flow initiation process. Flow over finite topography in a barotropic fluid is then considered (Figure 1.1b). This requires a modification of traditional contour dynamics so that we can apply appropriate matching conditions on the circular boundary of the topography. Next, two-layer simulations of flow over a small (quasi-geostrophic) topographic feature are made (Figure 1.1c). Finally, a quasi-geostrophic two-layer model of flow over a topographic feature of finite height with the geometry described above is considered (Figure 1.1d), where we again apply our modification to the contour dynamics method. The steady solution in Chapter 3 helps in our understanding the results of the time-dependent simulations.

In summary, in Chapter 2, we extend Ingersoll's (1969) model to flow on the $\beta$-plane. In Chapter 3, we consider two steady models of flow over finite topography on the $\beta$-plane, a barotropic model and a two-layer model. In Chapter 4, we develop a hierarchy of models of the initial value problem of flow over topography. In Chapter 5 , we summarize the results. 


\section{Chapter 2}

\section{Frictional Taylor columns on the $\beta$-plane}

\subsection{Introduction}

Taylor (1917) showed that flow of a slow, steady, inviscid fluid in a rapidly rotating tank is independent of the coordinate parallel to the axis of rotation; the fluid moves about in columns whose axes are parallel to the rotation vector. Therefore, when a fluid is obstructed by an isolated obstacle that has height less than the depth of the fluid, it tends to go around instead of over the obstacle. This phenomenon was observed in the laboratory by Taylor (1923) and was later dubbed a Taylor column (Hide, 1961). In laboratory experiments, the trapped fluid is stagnant, suggesting that friction is important; hence, the fluid is spun down within the Taylor column. Once closed streamlines form, the potential vorticity-streamfunction relationship is no longer constrained by upstream conditions. In purely inviscid theory, it is generally assumed that the conditions within the closed streamlines are the same as those upstream; we call these solutions the inertial solutions. Ingersoll (1969) showed that in the presence of even the smallest friction, the solution should approach a situation in which the fluid is stagnant within closed streamlines, and frictional and inertial effects both have order one importance. He constructed analytic solutions for flow over a flat-topped obstacle in the limit of vanishing viscosity under the quasi-geostrophic 
approximation. He discussed solutions in which fluid is trapped over the topography in a Taylor column and is spun down through the action of bottom drag. The flow outside of the Taylor column is exactly inviscid. The solutions are set up over the viscous spin-up time, which is much longer than the advection time for oceanic flow regimes. The resulting solutions qualitatively agree with laboratory experiments.

Johnson (1983), on the other hand, studied solutions in which trapped fluid is composed of fluid that originates over the topography before the flow has been turned on. He assumed that the steady state is set up over an advective time-scale. This process is discussed further in Chapter 4. Johnson (1983) showed that for flow over a right circular cylinder his solutions are identical to Ingersoll's solutions. If his assumptions are correct, then the solution to the frictional problem can be set up over a time scale much shorter than the spin up time, and still satisfy the conditions necessary for the fluid within the Taylor column to be stagnant.

Both Ingersoll (1969) and Johnson (1983) suggested ways in which to complete the problem of inviscid flow over topography when closed streamlines have formed by constraining the stream function-potential vorticity relationship within closed streamlines to be other than what exists upstream. In Johnson's (1983) solutions, the closure for the inviscid problems comes from finding the solution to the initial value problem such that the maximum amount of fluid that originated over the topography is retained there. Johnson (1983) showed that the maximum retention solution is the same as the solution found in the inviscid limit of the frictional problem [Ingersoll's (1969) type of solution] when the background flow is uniform or has negative linear shear. For negative shear the Taylor column is elongated along the $y$ axis. For positive background shear, the two solutions differ. The maximum retention solution is by construction wholly over the topography and the trapped fluid is contained within an ellipse with major axis parallel to the $x$ axis, while the Taylor column in the Ingersoll type solution extends to infinity along the $x$ axis. For all 
the cases that Johnson (1983) considered, for the Ingersoll type solutions the stagnation points of the flow are either at infinite $x$ or on the boundary of the topography. For smooth topography the maximum retention solutions do not exist because all fluid elements originate over the topography which is everywhere. In this chapter, an integral equation is found that can be solved to find the general solution of the inviscid limit of the frictional problem, Ingersoll's type of solution, and it is applied numerically. We extend Ingersoll's (1969) solution by including the $\beta$-effect.

We use the inviscid solutions that McCartney (1975) found on the $\beta$-plane as a starting point for our analysis. Like Johnson's (1983) solutions for positive background shear, the solutions that we find on the $\beta$-plane for westward flow are elongated in the $x$ direction, while for eastward flow they are slightly elongated in the $y$ direction, similar to Johnson's (1983) negative shear solutions.

To summarize what is included in this chapter: the inviscid limit of the frictional problem is constructed for flow over several shapes of bumps, and for flow over right circular cylinders with the inclusion of the $\beta$-effect, extending Ingersoll's (1969) solutions by numerically solving an integral equation. First the formulation of the problem is reviewed. Then the numerical technique is developed which is used to find the boundary of the stagnant region of fluid over the bump. Solutions of flow on the $f$-plane for several different topographic shapes are discussed. Solutions when $\beta$ is non-zero are discussed for both eastward and westward flow over a right circular cylinder. Finally, a discussion of the structure of the solutions is given as well as speculations on properties of the solutions under more general circumstances. 


\subsection{Model formulation}

Under the quasi-geostrophic approximation, the governing equations of the flow in steady state in a barotropic fluid are

$$
J(\psi, q)=\frac{1}{\tau} \nabla^{2} \psi
$$

where

$$
q=\frac{f_{0} h(x, y)}{H}+\nabla^{2} \psi+\beta y .
$$

Here, $\psi$ is the streamfunction of the flow where $\psi_{x}=v$ and $\psi_{y}=-u, h$ is the bottom topography, $f_{0}$ is the Coriolis parameter, $\beta=f_{y}$ the change in the Coriolis parameter with latitude, $H$ is the total depth of the fluid, and $\tau$ is the spin up time, measuring the effect of bottom drag on the flow. When $\tau \rightarrow \infty$ there are two possible solutions, $\psi=$ constant or $q=Q(\psi)$ where the relationship between the two fields is determined by upstream conditions. The solution with $q=$ constant corresponds to Rhines and Young's (1982) solution where the potential vorticity mixing acts within closed streamlines to make the potential vorticity constant; this solution is not included here because we are interested in situations in which bottom drag dominates. When closed streamlines appear in the flow, Ingersoll shows that $\psi=$ constant is required within closed streamlines when there is the smallest amount of bottom drag. This condition is equivalent to Rhines and Young's (1982) solution $q=$ constant when the topography has constant height and the flow is on the f-plane. Outside of closed streamlines the potential vorticity-stream function relationship is determined from upstream conditions.

Ingersoll's (1969) argument is based on the assumption that the closure for the inviscid problem comes from finding the inviscid solution by taking the inviscid limit of the frictional solution. This argument is examined below. When $\tau$ is finite, 
integrating 2.1 along a closed streamline results in

$$
\oint_{\psi=\text { constant }} \nabla \psi \cdot \hat{n} d l=0
$$

The circulation around closed streamlines must be zero. Since the velocity along the streamline does not change sign, the velocity must be zero within the closed streamline. Ingersoll (1969) argued that it can be seen from 2.1 that the vorticity $\nabla^{2} \psi$ must be order one everywhere, although there may be layers across which it changes abruptly. Thus there cannot be any shear layers, and the velocity must be continuous, with a correction proportional to a power of $1 / \tau$. Ingersoll (1969) showed that this holds when Laplacian friction is also included. We are thus left with the condition that the velocity on the closed streamline must be zero. He suggested that this can be understood by considering the Bernoulli function $B$. To first order, the Bernoulli function is given by the pressure and it must be zero at the stagnation point. Thus, along the boundary of the Taylor column, $B=\frac{1}{2}|\nabla P|^{2}=0$ where $P$ is the first order pressure (in a Rossby number expansion). This implies that velocity must be zero along the closed streamline. Therefore, not only must the streamfunction be constant, but the tangential velocity must be zero at every point on the boundary of the spun down region. For a consistent solution, this boundary must be the outermost closed streamline.

When $\tau>>1$ (the inviscid limit) and there is uniform flow upstream, the potential vorticity upstream of the obstacle will be given by

$$
q=\beta y
$$

The potential vorticity-streamfunction relationship is fixed by this upstream condition, and

$$
q=-\beta \psi / U
$$


where $U$ is the upstream velocity. This relationship is valid for all fluid parcels that originate upstream but is not necessarily valid for fluid parcels that are contained within closed streamlines.

To summarize, for $\tau>>1$, under the quasi-geostrophic approximation, outside of closed streamlines, we must solve 2.3 while satisfying the condition of no flow on any closed streamlines.

\subsection{Numerical technique}

The solution outlined above can be found numerically as an integral equation. The method is similar to the method that Meacham (1988) used to find a steadily translating vortex pair on the $\beta$-plane. It has been modified here for application to this physically different problem. When the flow is inviscid, We let $\partial D$ be the boundary of the Taylor column, then 2.3 becomes

$$
\nabla^{2} \psi+\frac{b}{V} \psi=-\frac{h}{\epsilon}+y
$$

where

$$
\begin{gathered}
\psi=-V y \quad|\mathbf{x}| \rightarrow \pm \infty \\
\psi=C \quad|\mathbf{x}| \in \partial D
\end{gathered}
$$

and

$$
\frac{\partial \psi}{\partial n}=0 \quad|\mathbf{x}| \in \partial D
$$

where $\hat{n}$ is the direction normal to the boundary. We have nondimensionalized 2.2 such that $x, y$ are scaled by $L$, the length scale of the topography, $\psi$ is scaled by $|U| L$, and $h$ is a non-dimensional function scaled by $H$, the total depth of the fluid. We let $h$ be order $\epsilon$, where $\epsilon=|U| / f_{0} L$ is the Rossby number, and the flow is quasigeostrophic. There are three parameters in the problem, $b=\beta L^{2} /|U|$, the ratio of 
the long Rossby wave speed with the background flow velocity, $h_{0} / \epsilon$, the height of the topography relative to the Rossby number, and $V$, the direction of the flow; $V=1$ for eastward flow and $V=-1$ for westward flow. The constant $C$ determines the value of the streamfunction on $\partial D$ and where the streamline that comes into the stagnation point from the exterior originates upstream. $\partial D$ is not contained within any closed streamlines and the boundary $\partial D$ must be determined in the course of the solution.

The numerical technique used is a boundary integral technique related to contour dynamics, reducing the problem to solving a one-dimensional problem on the boundary of the Taylor column $\partial D$, and then iteratively solving for $\partial D$. The procedure can be summarized as follows: first the values of $b$ and $h_{0}$ as well as the shape of the topography and the direction of the flow are chosen. Next, an initial guess for the shape of $\partial D$ and for the constant $C$ are made. Then 2.4 is solved for the stream function on the boundary $\partial D$ subject to the boundary conditions 2.5 and 2.7. Finally, using the variations of $\psi$ on $\partial D$, the boundary is adjusted such that the variation in $\psi$ is reduced on the boundary to satisfy 2.6 . With this new estimate, the procedure is iterated until $(\psi-C)^{2}$ averaged over $\partial D$ is within a certain tolerance. The resulting boundary is not necessarily the correct one, because the boundary conditions can be satisfied on $\partial D$ while $\partial D$ is contained within a closed streamline. $C$ must be adjusted until there are no other closed streamlines in the flow besides $\partial D$. Determining if the solution contains any other closed streamlines is non-trivial, but we assume that the correct solution is that which has the largest area and that it is uniquely determined. This assumption is based on experience with the numerical solutions.

The solutions for which 2.4, 2.5, 2.6, and 2.7 are satisfied for an intermediate value of $C$ are solutions to an initial value problem of fluid which begins at rest over the topography which is then set into motion by a background flow field being turned on at $t=0$ where some of the fluid which originated over the topography is swept downstream while some remains over the topography. We name these intermediate 
retention solutions. Johnson's (1983) maximum retention solution corresponds to the situation in which all of the fluid remains over the topography. The intermediate retention solutions contain closed streamlines outside $\partial D$ and we show in Chapter 4 that these different solutions can be approximately realized in the initial value problem. They are valid solution to the problem of flow over topography for times short compared to the viscous spin up time $\tau$ are so are not relevant to the discussion here.

In order to derive the integral equation, we need the Green's function for the problem which is defined by the solution to the following partial differential equation:

$$
\nabla^{2} G\left(\mathbf{x}, \mathbf{x}^{\prime}\right)+\frac{b}{V} G\left(\mathbf{x}, \mathbf{x}^{\prime}\right)=\delta\left(\mathbf{x}-\mathbf{x}^{\prime}\right)
$$

The Green's function depends on the value of $b$ and $V$. When $b$ is zero,

$$
G\left(\mathbf{x}, \mathbf{x}^{\prime}\right)=\frac{1}{2 \pi} \ln \left(\left|\mathbf{x}-\mathbf{x}^{\prime}\right|\right)
$$

When $V=-1$ (westward flow), the Green's function once again depends on $\left|\mathbf{x}-\mathbf{x}^{\prime}\right|$ and is given by

$$
G\left(\mathbf{x}, \mathbf{x}^{\prime}\right)=-\frac{1}{2 \pi} K_{0}\left(b^{\frac{1}{2}}\left|\mathbf{x}-\mathbf{x}^{\prime}\right|\right)
$$

where $K_{0}$ is the modified bessel function of the second kind and zero order. When $V=+1$ (eastward flow), 2.8 is a wave-like Helmholtz equation and stationary Rossby waves with wave number $b^{1 / 2}$ exist. Johnson (1977) derived the Green's function in this case. It has the property that there is no upstream energy propagation and no longer depends simply on $\mathbf{x}-\mathbf{x}^{\prime}$ but is given by

$$
G\left(\mathbf{x}, \mathbf{x}^{\prime}\right)=\frac{1}{4} Y_{0}\left(b^{1 / 2} R\right)+\frac{1}{\pi} S\left(b^{1 / 2} R, \Theta\right)
$$

where

$$
S(s, \alpha)=\sum_{k=0}^{\infty} \frac{\cos [(2 k+1) \alpha] J_{2 k+1}(s)}{2 k+1}
$$

and

$$
\left(x-x^{\prime}, y-y^{\prime}\right)=(R \cos \Theta, R \sin \Theta) .
$$


In practice, the series is truncated at a finite value of $k$.

A particular solution $\psi^{(a)}$ of 2.4 is found subject to 2.5. This solution is defined as the inertial solution, independent of friction, which is the complete solution when closed streamlines have not formed. This solution was found by McCartney (1975) for the right circular cylinder, for both positive and negative $V$. In general it can be found by applying Green's theorem

$$
\psi^{(a)}=-V r \sin \theta+\int G\left(\mathbf{x}, \mathbf{x}^{\prime}\right) h\left(\mathbf{x}^{\prime}\right) d x d y .
$$

The homogeneous solution $\phi$ that allows $\psi$ to satisfy the boundary conditions 2.6 and 2.7 must be determined where

$$
\psi=\psi^{(a)}+\phi
$$

Applying Green's theorem to 2.4 gives the following integral equation

$$
\oint_{\partial D} \phi\left(\mathbf{x}^{\prime}\right) \frac{\partial G\left(\mathbf{x}, \mathbf{x}^{\prime}\right)}{\partial n^{\prime}} d s^{\prime}-\oint_{\partial D} G\left(\mathbf{x}, \mathbf{x}^{\prime}\right) \frac{\partial \phi}{\partial n^{\prime}}\left(\mathbf{x}^{\prime}\right) d s^{\prime}+\phi(\mathbf{x})=0
$$

for any point $\mathbf{x}$ not on the boundary $\partial D$. Thus if we knew both $\partial D$ and $\phi$ on $\partial D$, then $\phi$ would be known everywhere. On the boundary, there is a singularity when $\mathbf{x}^{\prime}=\mathbf{x}$, and the integral does not exist. We use the principal value of the integrals in 2.10 which is a reasonable limit of the integrals as $\mathbf{x}$ approaches $\partial D$. Because $x$ is on $\partial D$, there is a factor of $1 / 2$ introduced due to the fact that the integral is completed by doing a half circle (instead of a full circle) around the singular point $x$. We use the principal value of the contour integrals in 2.10 as the limit as $\mathbf{x}$ approaches $\mathbf{x}^{\prime}$ on $\partial D$. Therefore, when $x$ is on $\partial D 2.10$ is modified:

$$
\oint_{\partial D} \phi\left(\mathbf{x}^{\prime}\right) \frac{\partial G\left(\mathbf{x}, \mathbf{x}^{\prime}\right)}{\partial n^{\prime}} d s^{\prime}-\oint_{\partial D} G\left(\mathbf{x}, \mathbf{x}^{\prime}\right) \frac{\partial \phi}{\partial n^{\prime}}\left(\mathbf{x}^{\prime}\right) d s^{\prime}+\frac{1}{2} \phi(\mathbf{x})=0
$$

for points on the boundary. The normal derivatives are taken outward from the boundary of the Taylor column, and $d s$ is the element of arc along the boundary. We use the boundary condition 2.7 and substitute $\partial \phi / \partial n=-\partial \psi^{(a)} / \partial n$ so that 2.11 is a linear inhomogeneous integral equation for $\phi$ on $\partial D$ as a function of arc length. 
This integral equation 2.11 is similar to the approximate equation that Johnson (1983) used to find the boundary of a Taylor column in the limit of large positive shear. In Section 2.4 we show that the choice of using the principal value of the integral is correct by applying 2.11 to the analytic solution that Ingersoll (1969) found.

Once $\phi$ is known on the boundary, the total streamfunction can be calculated everywhere using 2.10. In order to implement the procedure numerically, the boundary is represented by $N+1$ points $\{\mathbf{x}: 0 \leq i \leq N\}$ lying on a single closed curve. To find $\phi$ on $\partial D,(2.11)$ can be discretized and put in the form of a system of simultaneous linear algebraic equations for the $N+1$ unknowns $\phi\left(x_{i}\right), 0 \leq i \leq N$, which can then be solved numerically. This procedure is described in Appendix A. The constant $C$ must be determined as described above. Once a solution is found for a given topographic shape and height, the $C$ for the desired solution can be found by changing $C$ to make $\partial D$ larger until for a given value of $C$, a solution for $\partial D$ cannot be found. Once a solution for a given topographic height is found, then the height can be increased slightly, and a solution can be found for the new topographic height. Likewise, $b$ can be increased slowly and each successive solution found by using the last known solution as an initial guess. The solution found analytically by Ingersoll for flow over a right circular cylinder on the $f$-plane is used as a starting point for the procedure.

The method as presented up to this point could be used for any shape of topography as long as $\psi^{(a)}$ could be determined. In fact, one could construct a grid of $\psi^{(a)}$ over the topography so that it would not have to be determined at each iteration step. For axisymmetric topography, and for flow on the f-plane or westward flow on the $\beta$-plane,

$$
\psi^{(a)}=H(r)-V y
$$

where $H(r)$ is a function depending only on $r$. This simplifies the calculation of the inertial stream function $\psi^{(a)}$ even further. 
This numerical procedure works well when something is known about the location of the boundary of the Taylor column. However, when there is no such information, it has not been possible to find a solution. There are also some indications that the solutions can only be found when the topography is of compact support (it is non-zero in a finite region).

From the numerical solutions found, it appears that the stagnation point of the Taylor column is located on the line where the topography vanishes. On the f-plane, Ingersoll (1969) pointed out that for his solutions, the Taylor column cannot exist in a place outside the support of the topography because the solution to Laplace's equation is the trivial solution when both the function and its derivative vanish on a finite line. One can see this most easily by finding the solution via a Taylor series expansion beginning along that line. It is not obvious however, why why the stagnation point is located at the edge of the topography, or where the location of the stagnation point would be for smooth topography.

One way to try to answer this question is to construct the stream function locally about the stagnation point through a Taylor series expansion, satisfying 2.4 in the process. We consider flow on the f-plane. For axisymmetric topography, the stagnation point is located at $x=0$ and the flow is symmetric about the $y$ axis. The line defined by $\psi=C$ is composed of two parts: the Taylor column boundary and the line originating at the stagnation point and ending at $\pm \infty$. To construct the Taylor series expansion about the stagnation point, we need to make some assumptions about the shape of these lines locally about the stagnation point. For this discussion we reference $x$ and $y$ to the location of the stagnation point. The simplest situation is to let $x=a y$ for the line that originates at $\pm \infty$. This implies that the flow field has normal stagnation point behavior. We assume that the Taylor column boundary is shaped like a parabola $\left(x=c y^{2}\right)$ locally, using intuition developed from Ingersoll's solution. With these two assumptions and the requirement that the velocity must 
vanish on the Taylor column boundary, a consistent solution can only be found when the topography vanishes at the stagnation point, allowing a different Taylor series expansion over and away from the boundary of the topography. These ideas can be extended to the $\beta$-plane, although the condition for the existence of the solution is somewhat more stringent than for the f-plane. The above argument suggests that the topography must be finite in extent for the solution to exist, but it is not conclusive both because the Taylor series expansion requires an a priori guess for the shape of the streamline, and the solution is not found globally, but only locally about the stagnation point.

\subsection{Taylor columns on the $\mathrm{f}$-plane}

The simplest topographic configuration is the one considered by Ingersoll (1969), the right circular cylinder (Figure 1.1a) where the topography $h$ is given by

$$
h= \begin{cases}0 & r>1 \\ h_{0} & r<1 .\end{cases}
$$

Although this topography is quite special, some physically important aspects of the flow can be discovered by considering this topographic configuration.

On the $\mathrm{f}$-plane $(b=0)$, this problem can be solved analytically. As Ingersoll (1969) found, when there are no closed streamlines in the flow, the inertial solution is given by

$$
\psi^{(a)}= \begin{cases}-\frac{h_{0}}{2 \epsilon} \ln r+r \sin \theta & r>1 \\ -\frac{h_{0}}{4 \epsilon}\left(r^{2}-1\right)+r \sin \theta & r<1 .\end{cases}
$$

where When the topographic height $h_{0}>2 \epsilon$ closed streamlines appear in the flow and the solution does not satisfy the additional boundary conditions. Ingersoll (1969) 
finds the solution of 2.4 subject to $2.5,2.6$, and 2.7 to be

$$
\psi= \begin{cases}-\frac{h_{0}}{2 \epsilon} \ln r+\frac{K}{2 \pi} \ln \left(\frac{r^{\prime}}{r_{c}}\right)+r \sin \theta & r>1 \\ -\frac{h_{0}}{4 \epsilon}\left(r^{2}-1\right)+\frac{K}{2 \pi} \ln \left(\frac{r^{\prime}}{r_{c}}\right)+r \sin \theta & r<1 \\ \text { constant } & r^{\prime}<1\end{cases}
$$

Here $r_{c}=1-2 \epsilon / h_{0}, K=\pi h_{0} r_{c}^{2} / \epsilon$, and $r^{\prime}$ is the radius referenced to $x=0, y=2 \epsilon / h_{0}$. The Taylor column is a circle with radius $r_{c}$ centered at $x=0$ and $y=2 \epsilon / h_{0}$. The boundary of the Taylor column coincides with a streamline of the inviscid solution (Figure 2.1), and the flow is westward. When the topography becomes tall, the Taylor column completely covers the topography approaching a circle with radius 1 . The limit of this solution for large values of $h_{0} / \epsilon$ is the solution of irrotational flow around a cylinder with exactly the circulation $(4 \pi)$ required to give a single stagnation point on the column boundary (e.g. Batchelor, 1967, Figure 6.6.1 (c)), and not irrotational flow around a cylinder with zero circulation. There is a pronounced left-right asymmetry (looking downstream) of the flow, which is characteristic of flow patterns found in laboratory experiments.

We now proceed to apply 2.11 to the solution in 2.12 for points on $\partial D$. Since $\psi^{(a)}$ is constant there, $\phi$ must also be constant, and can be taken outside of the integral. Also, $\partial \psi^{(a)} / \partial n=h_{0} r_{c} / 2 \epsilon$, and the total derivative must vanish on the boundary of the Taylor column so that $\partial \phi / \partial n=-\partial \psi^{(a)} / \partial n$ there. We do the integrals around the circular boundary of radius $r_{c}$ and we find that

$$
\oint_{\partial D} \frac{\partial G}{\partial n^{\prime}} d s^{\prime}=\frac{1}{2}
$$

and

$$
\oint_{\partial D} G d s^{\prime}=\frac{1}{2 \pi} \ln r_{c}
$$

Finally, solving 2.11 for $\phi$ on $\partial D$ we find

$$
\phi=\frac{h_{0} r_{c}^{2}}{2 \epsilon} \ln r_{c}
$$




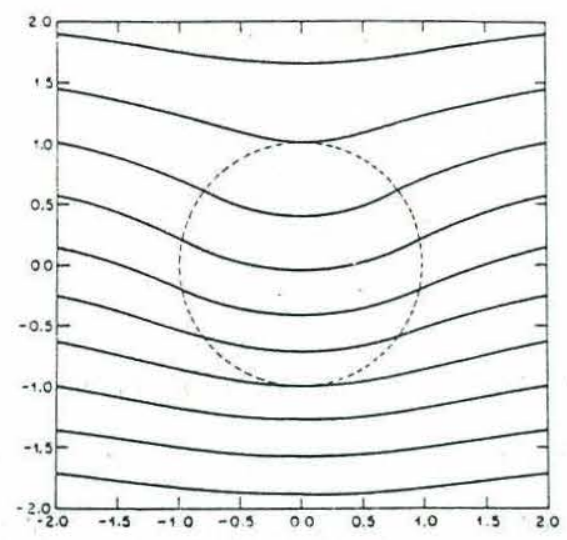

(a)

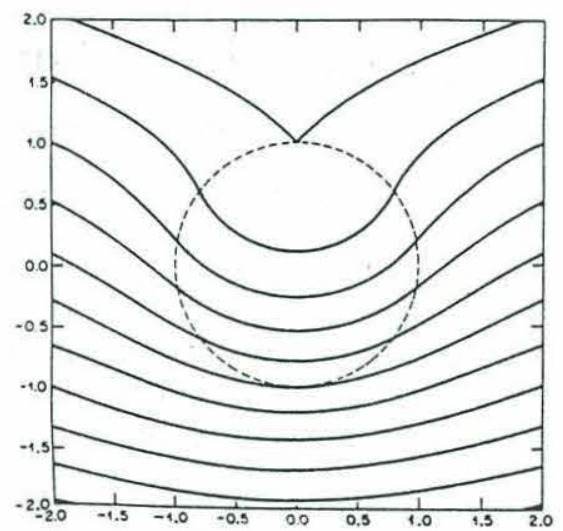

(b)

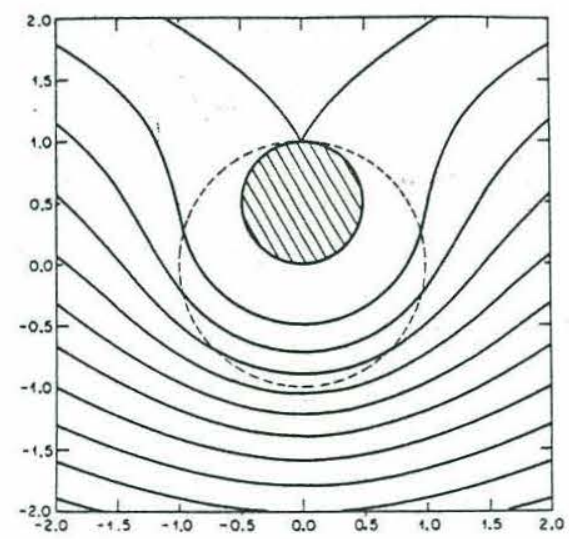

(c)

Figure 2.1: Streamlines for flow over a right circular cylinder of radius unity (dotted). Flow is going from right to left. The streamline contour interval is 0.2 . (a) No Taylor column, $h_{0} / \epsilon=1$. (b) No Taylor column at the critical height $h_{0} / \epsilon=2$. (c) $h_{0} / \epsilon=4$, Taylor column is contained within the circle and is hatched, after Ingersoll (1969). 
This is the correct answer for $\phi$ on $\partial D$ given from $2.12\left(\psi^{(a)}\right.$ is zero there). When we apply the numerical technique we find that $C=h_{0} r_{c}^{2} \ln r_{c} / 2 \epsilon$ as expected and the numerically determined Taylor column boundary is identical to Ingersoll's (1969). This result strengthens our justification of using the principal value of the integral in finding 2.11. The solution for this topographic configuration is found numerically to check the procedure, and it is identical to Ingersoll's (1969) solution.

The next most complicated topographic configuration is the right circular cone. Experience with the numerical solutions suggest that the stagnation point of the Taylor column is located on the edge of the topography. The topography is given by $h(r)=h_{0}(1-r) H(1-r)$ where $H(r)$ is the step function. In this case, the inertial solution is given by

$$
\psi^{(a)}= \begin{cases}-\frac{h_{0}}{\epsilon}\left(\frac{r^{2}}{4}-\frac{r^{3}}{9}\right)-r \sin \theta & r>1 \\ -\frac{h_{0}}{\epsilon}\left(\frac{1}{4}-\frac{1}{9}+\frac{1}{6} \ln r\right)-r \sin \theta & r<1 .\end{cases}
$$

When $h_{0} / \epsilon>16 / 3$ closed streamlines appear in the flow. The location of the first stagnation point is $r=3 / 4$. Solutions are found for values of $h_{0} / \epsilon$ for which the stagnation point of $\psi^{(a)}$ is located at a radius greater than one. As the topography becomes small, the Taylor column boundary becomes more and more elongated in the $y$ direction, giving a tear drop shape (Figure 2.2). The boundary of the Taylor column is not a streamline of the inviscid solution, unlike the solution found by Ingersoll (1969) which is given in 2.12 (Figure 2.3).

It is interesting to note that the slope in the topography reduces the magnitude of the circulation introduced over topography which has the same horizontal extent and overall height, but is flat-topped. Qualitatively we expect that the effect of slope in the topography is similar to the effect of introduction of positive circulation to Ingersoll's (1969) problem. As fluid parcels move up slope, their potential vorticity is decreasing, resulting in an increase in their negative relative vorticity to compensate for this. Thus the Taylor column is elongated perpendicular to the flow. This is 


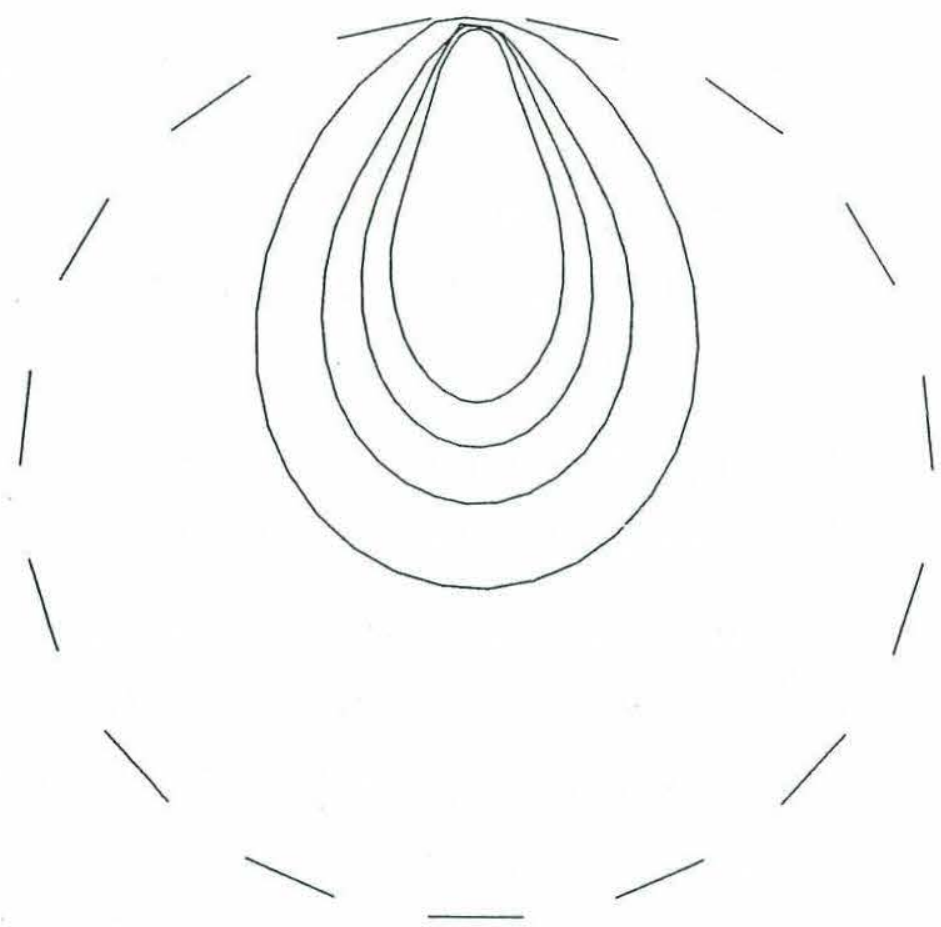

Figure 2.2: Boundary of the Taylor column for flow over a cone of radius unity (dashed) with flow going from right to left. $h_{0} / \epsilon=7,8,10,15$. The smallest boundary corresponds to the smallest topographic height. The Taylor column is tangent to the edge of the topography. 


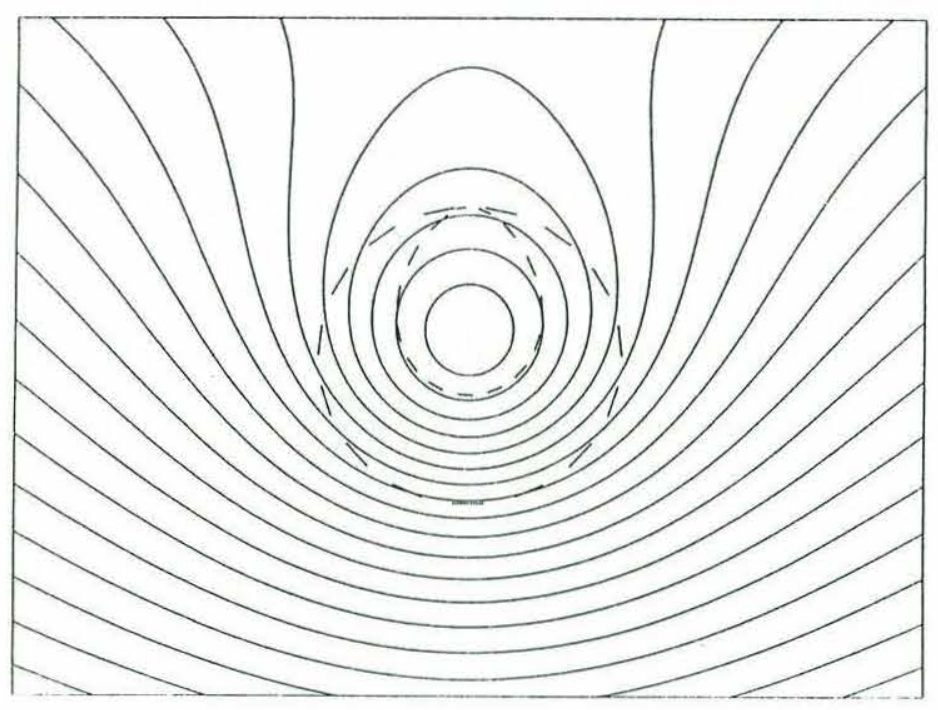

(a)

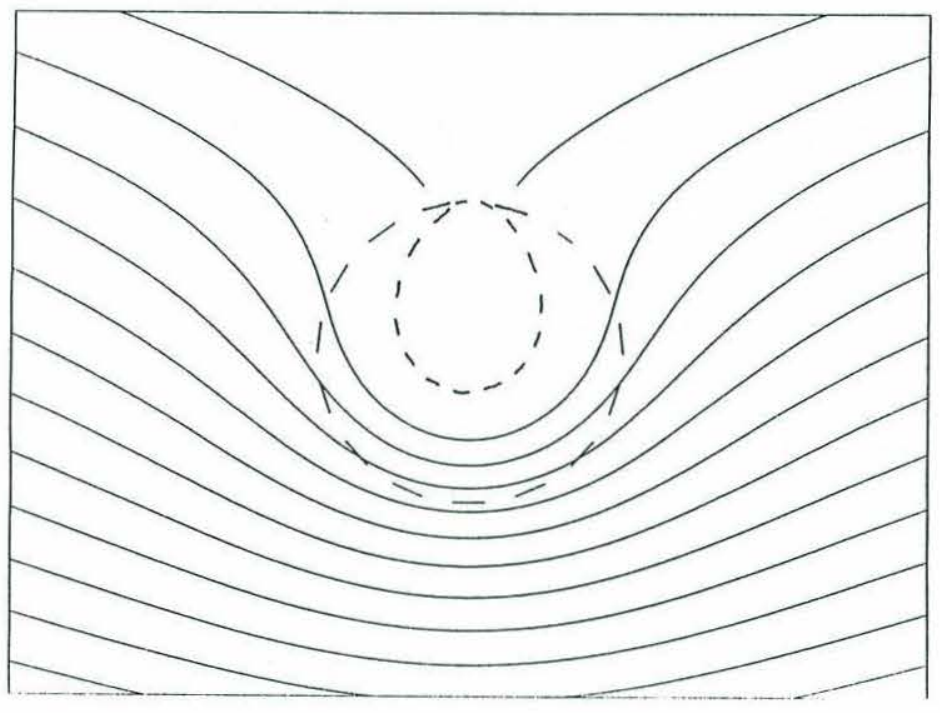

(b)

Figure 2.3: Streamlines for flow over a cone of radius unity (dashed) with flow going from right to left. $h_{0} / \epsilon=15$. The streamline contour interval is 0.4 . (a) The inertial solution $\psi^{(a)}$; the Taylor column boundary is dashed and is not a streamline of the inertial solution, and (b) the full solution. 
observed in the numerical solutions, and the solutions are similar to Johnson's (1983) solutions with negative shear.

It was not possible to find the solution for a value of topographic height for which the stagnation point of the inertial solution was located at $r<1$. This does not preclude the existence of the solution, but following the tendency of the solution to become narrower toward the stagnation point, one can imagine what would happen for smaller topographic heights than those shown in Figure 2.2. The Taylor column would become narrower, and possibly, the limit would be a line stretching from $r=1$ to some interior point. If this is true, it is not surprising that it was not possible to find solutions for smaller topographic heights. The solutions for flow over the cone are more difficult to find than for the right circular cylinder in the sense that a smaller value of $\alpha$, the relaxation parameter, is needed, as well as a better initial guess for the shape of $\partial D$.

Ingersoll (1969) found an approximate solution for flow over a cone when it is tall. Then the boundary of the Taylor column is given approximately by

$$
r_{c}(\theta)=1-\left[4 \epsilon / h_{0}(1-\sin \theta)\right]^{1 / 2} .
$$

In the limit of large topographic height, the shape of the Taylor column from 2.13 agrees more and more closely with the numerical solutions (Figure 2.4). One might expect qualitative agreement between the shape of the Taylor column shown for this example with more general topographic shapes that have a monotonic decrease in height from $r=0$ and are compact. The sense of circulation introduced by the changing topography would be to first order the same for a cone.

James (1980) found a similar solution to the ones seen in Figure 2.2 as the steady state solution from an initial value problem of flow over topography that was compact with a $\cos ^{2} r$ shape. The fluid was allowed enough time to spin down within the Taylor column. The structure of the flow is what would be expected from the 


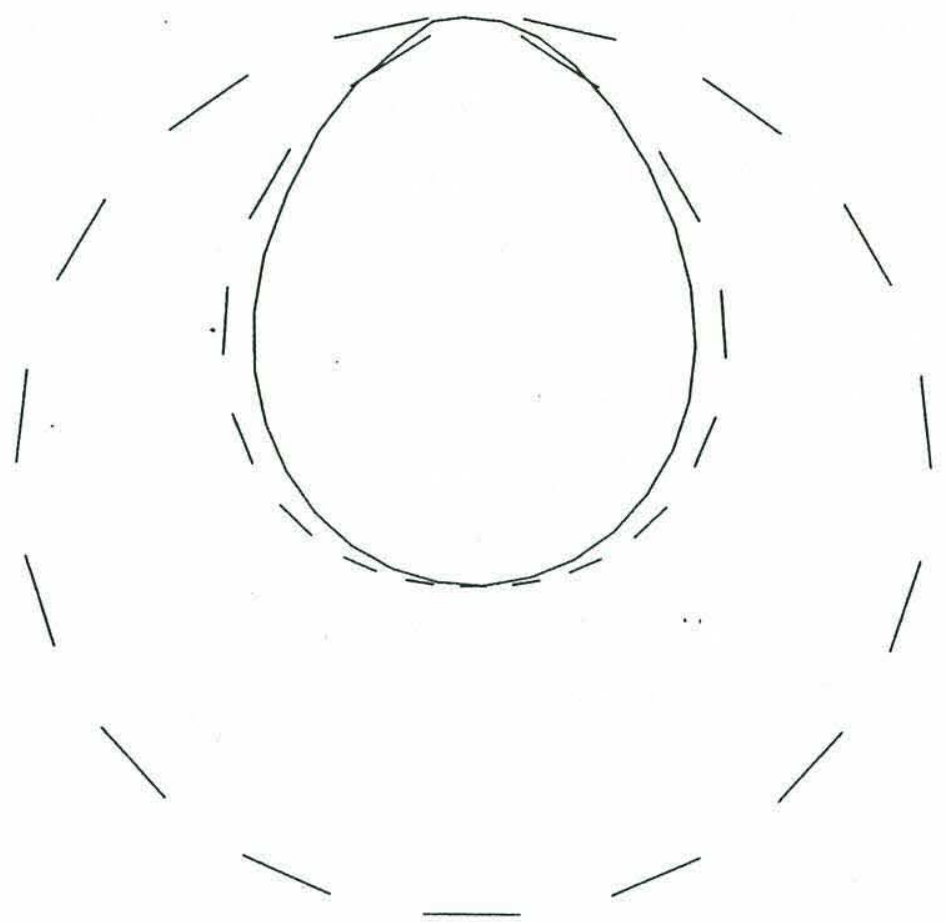

Figure 2.4: Boundary of the Taylor column for flow over a cone with radius unity (cirular dashed contour) and flow going from right to left. The solid contour corresponds to the numerical solution with $h_{0} / \epsilon=15$, while the dashed contour corresponds to Ingersoll's (1969) approximate solution, 2.13. 
theory presented above. However, it is difficult to determine the exact boundary of the stagnant region from his work, and he used a finite value of friction.

An attempt was made to find the solution when the topography falls off exponentially at large $r$. It was possible to find intermediate retention solutions in which the boundary conditions were satisfied, but closed streamlines appeared outside of $\partial D$. When $C$ was adjusted to allow $\partial D$ to become larger, the Taylor column became larger at each iteration step, and the algorithm did not appear to be converging toward a solution. This failure, together with the failure to find solutions for flow over a cone when the stagnation point of the inertial stream function is located at $r<1$ and the evidence from the Taylor series expansion about the stagnation point, all suggest that the topography must go to zero at a finite $r$ for a well-behaved solution to exist. It seems that the problem that Ingersoll (1969) posed for the boundary of the Taylor column in the inviscid limit of the frictional problem will not converge on smooth topography; perhaps the friction enters in a more complex way than simply an additional constraint on the inviscid solution.

\subsection{Taylor columns in a westward flowing current on the $\beta$-plane}

Westward flow $(V=-1)$ on the $\beta$-plane is characterized by the length scale $1 / b^{1 / 2}$, the inertial boundary current scale. For westward flow, the stationary Rossby waves are evanescent, so the effect of the topography is localized. Over a right circular cylinder, the inertial solution was given by McCartney (1975) as

$$
\psi^{(a)}=-r \sin \theta+\frac{h_{0}}{\epsilon b^{1 / 2}} \begin{cases}I_{1}\left(b^{1 / 2}\right) K_{0}\left(b^{1 / 2} r\right) & r>1 \\ -K_{1}\left(b^{1 / 2}\right) I_{0}\left(b^{1 / 2} r\right)+\frac{1}{b^{1 / 2}} & r<1 .\end{cases}
$$


The critical height above which closed streamlines form was found by McCartney (1975) and is given by

$$
h_{c}=\left[I_{1}\left(b^{1 / 2}\right) K_{1}\left(b^{1 / 2}\right)\right]^{-1},
$$

and is shown in Figure 2.5. For small $b$ the solution reduces to the f-plane result as would be expected, while for large $b$, the critical height becomes large. As the effect of $\beta$ increases, the perturbation to the background flow is more confined to the northernmost extent of the topography, requiring a larger bump for closed streamlines to form. Figure 2.6 shows a series of Taylor column boundaries for a fixed value of topographic height, while $b$ is varied. The aspect ratio of the Taylor column becomes smaller as $b$ becomes larger. The limited extent of the Taylor column is seen as $b$ becomes large. A comparison between the inertial solution and the frictional solution is seen in Figure 2.7. The difference is only seen out to a distance $1 / b^{1 / 2}$ away from the Taylor column, and in Figure 2.8 we show that the boundary of the Taylor column is not a streamline of the inviscid solution.

The circulation induced by the $\beta$-effect is in the opposite sense of the circulation induced by the topography. As a fluid parcel moves south because of the negative circulation induced by the topography, it gains positive relative vorticity to compensate for the change in latitude. The two effects work to compensate each other so that the Taylor column has limited north-south extent and is confined to the northern-most part of the topography. The Johnson (1983) solutions for positive shear are also elongated in the $x$ direction.

\subsection{Taylor columns in an eastward flowing current}

In an eastward flowing current, stationary Rossby waves are generated at the topography. These waves cause upstream-downstream asymmetry in the flow. Because of this, there is an associated drag on the topography, and the Taylor column is no longer 


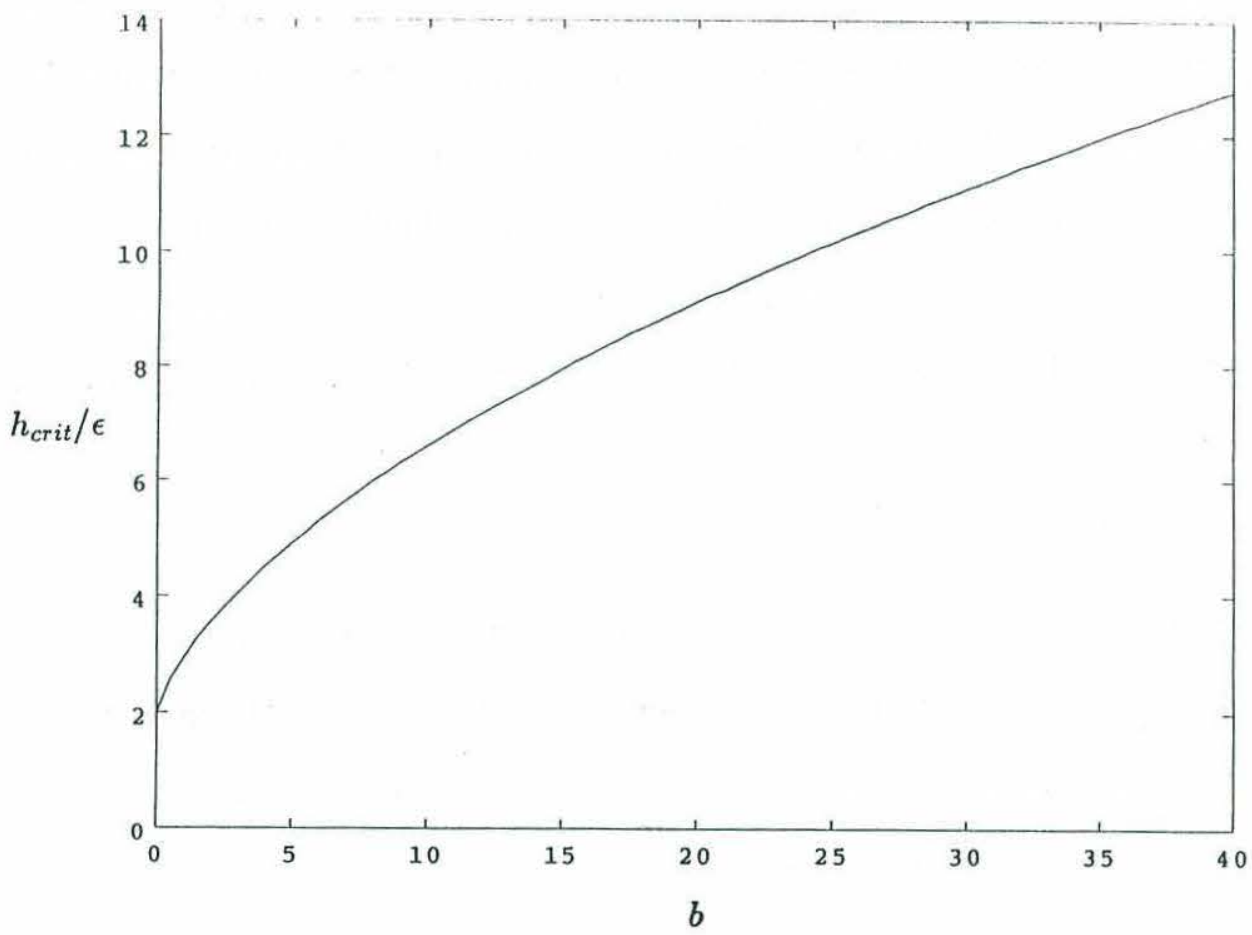

Figure 2.5: Critical height for closed streamlines to form for westward flow over a right circular cylinder on the $\beta$-plane as a function of $b$. In the limit of $b \rightarrow \infty$ the critical height goes to $\infty$, while in the f-plane limit, $(b=0)$ the critical height is 2 , after McCartney (1975). 


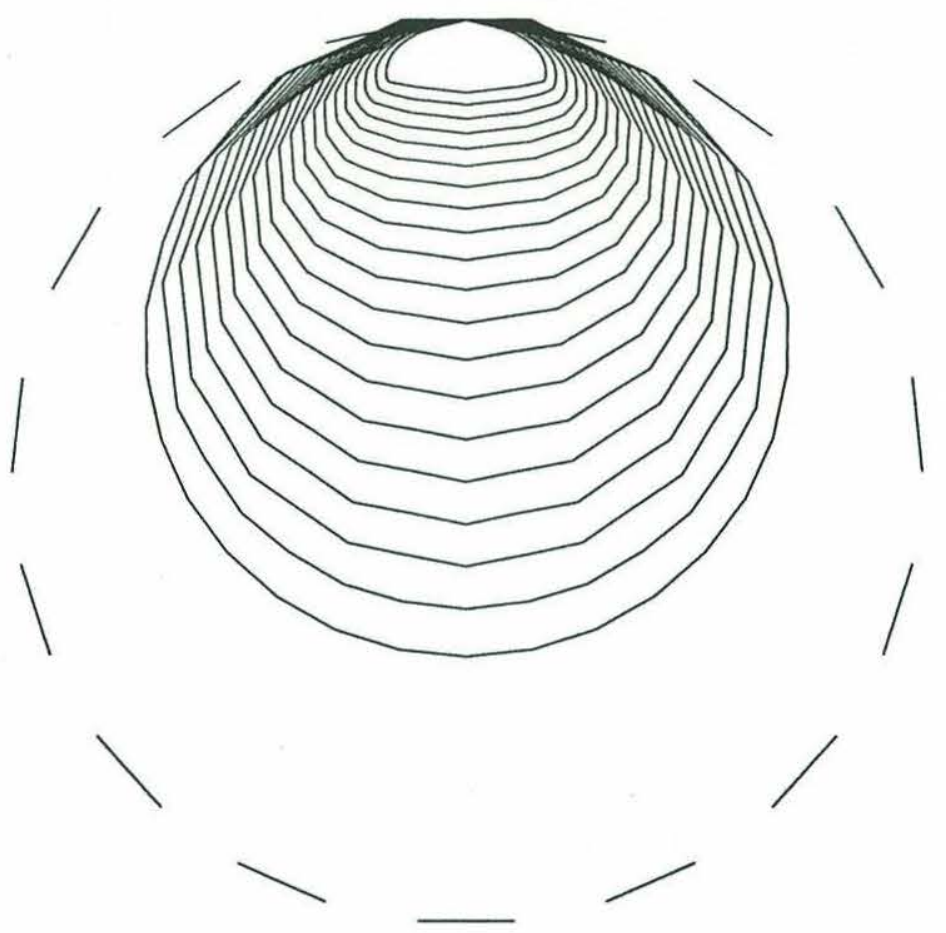

Figure 2.6: Boundary of the Taylor column when $h_{0} / \epsilon=8$ and $b$ varies from .5 up to 10 in increments of 0.5 . Flow is going westward, from right to left. The dashed circle is the boundary of the topography. The largest Taylor column corresponds to the smallest value of $b$ while the smallest Taylor column correspond to the largest. The Taylor column is tangent to the edge of the topography, and becomes elongated in $x$ for increasing $b$. 


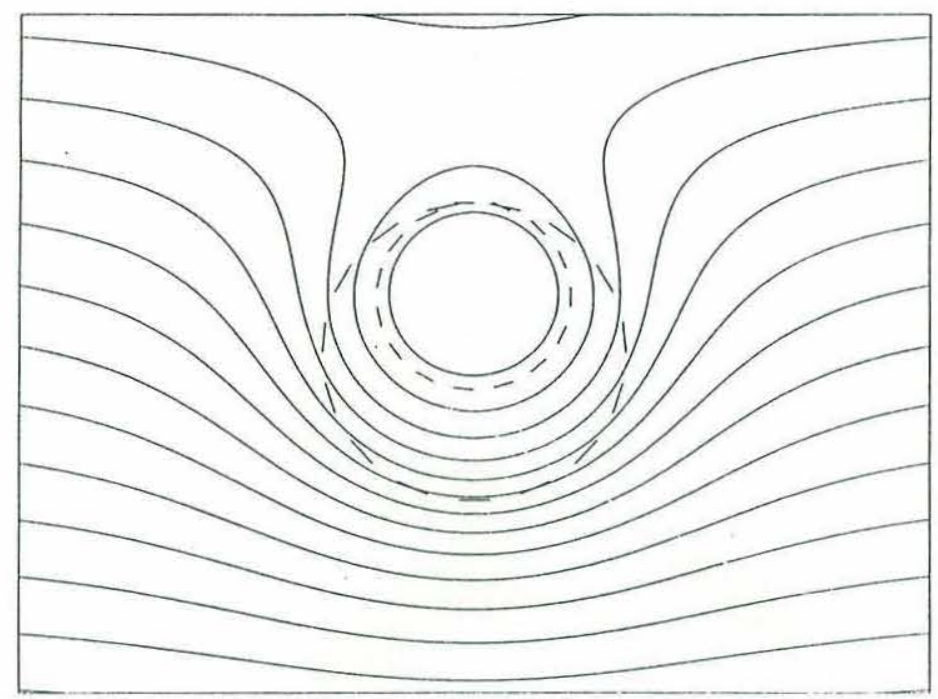

(a)

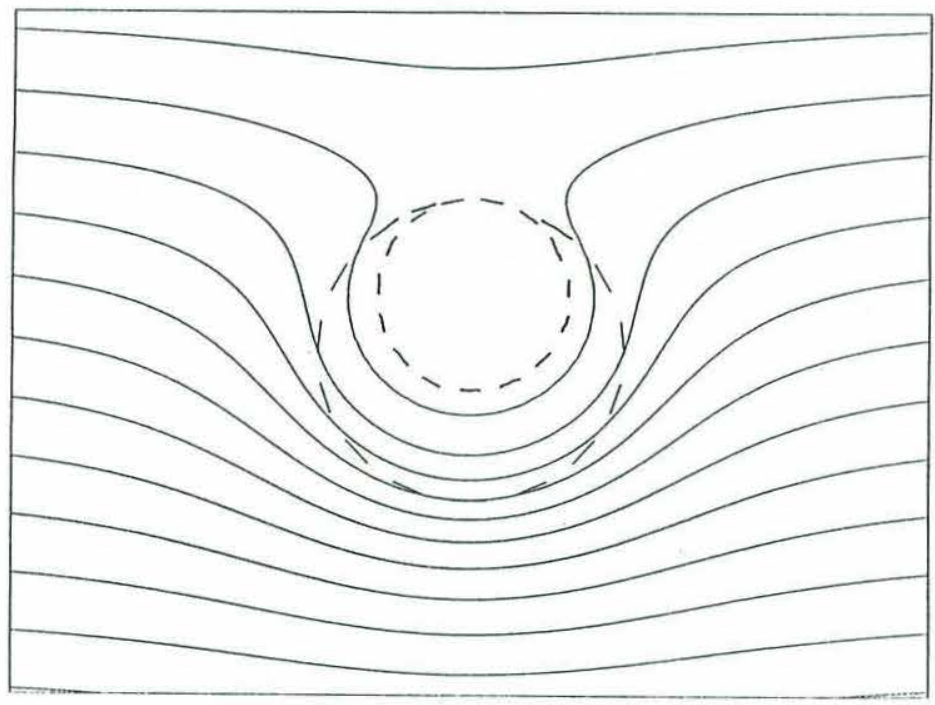

(b)

Figure 2.7: Streamlines for westward flow over a right circular cylinder of radius unity (dashed), $h_{0} / \epsilon=8$, and $b=1$. The streamline contour interval is 0.4 . (a) The inertial solution $\psi^{(a)}$; the Taylor column boundary is dashed. (b) The full solution. The effect of the Taylor column is confined near the topography. 


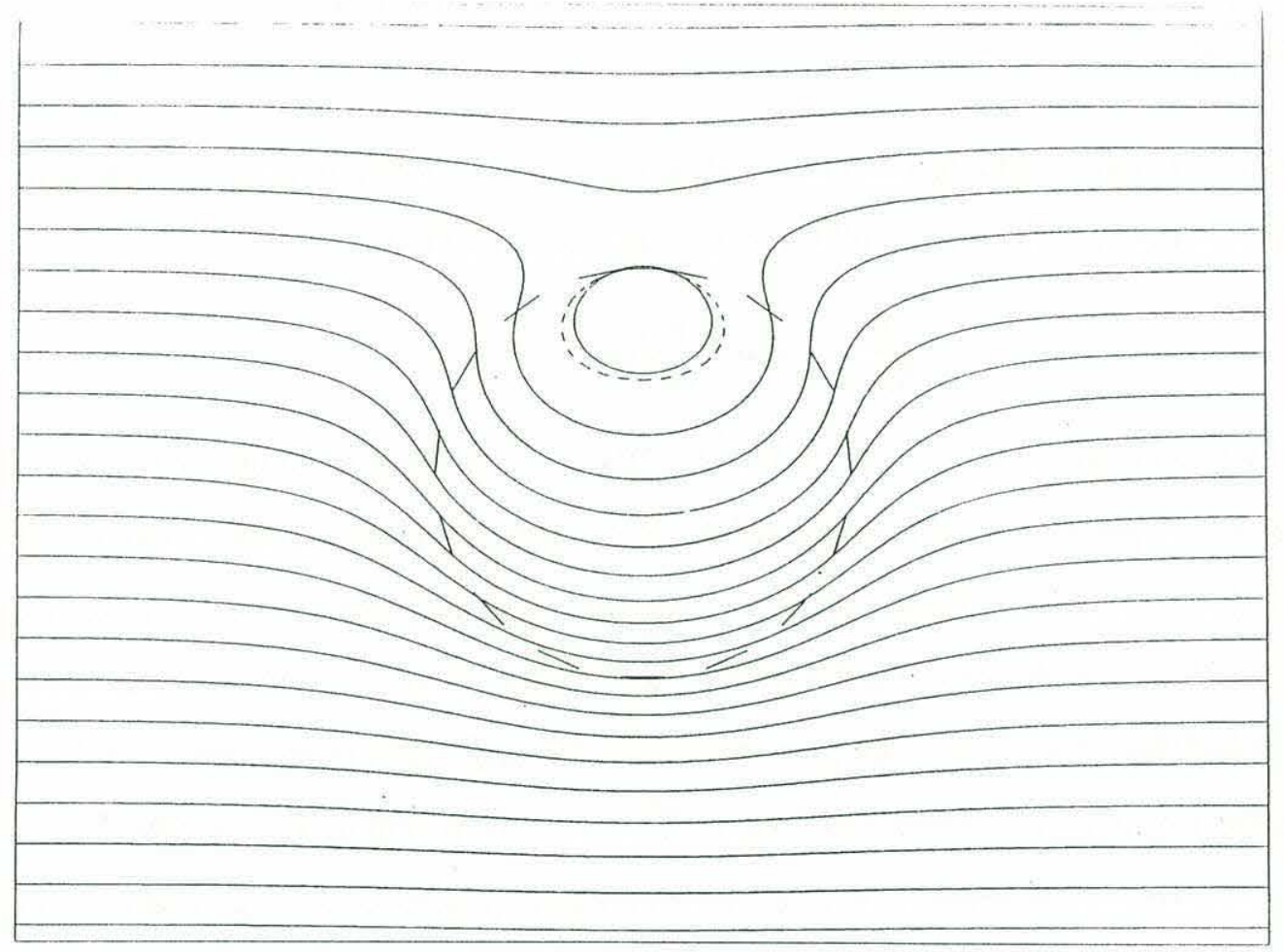

Figure 2.8: Streamlines for westward flow over a right circular cylinder of radius unity (dashed), $h_{0} / \epsilon=8$, and $b=3$. The streamline contour interval is 0.4 . The inertial solution $\psi^{(a)}$, the Taylor column boundary is dashed and is not a streamline of the inertial solution. 
located symmetrically about the $y$ axis. With these considerations in mind, solutions are found for eastward flow over a right circular cylinder. The inertial solution was found by McCartney (1975) and is given by

$$
\psi^{(a)}=-r \sin \theta-2 \frac{h_{0}}{\epsilon b^{1 / 2}} J_{1}\left(b^{1 / 2}\right) S(r, \theta)-\frac{h_{0}}{\epsilon b^{1 / 2}} \begin{cases}\frac{\pi}{2} J_{1}\left(b^{1 / 2}\right) Y_{0}\left(b^{1 / 2} r\right) & r>1 \\ \frac{\pi}{2} Y_{1}\left(b^{1 / 2}\right) J_{0}\left(b^{1 / 2} r\right)+\frac{1}{b^{1 / 2}} & r<1\end{cases}
$$

where $S(r, \theta)$ is given in 2.9 .

The critical height when closed streamlines first form is given in general by finding the critierion such that $u^{(a)}$ and $v^{(a)}$ are zero or that

$$
\frac{\epsilon}{h_{\text {crit }}}=\max \left[\frac{\epsilon}{h_{0}}\left(\psi_{y}^{(a)}+1\right)\right]
$$

subject to the constraint $\psi_{x}=0$ (Johnson, 1977). The critical height can be found easily on the $f$-plane and for westward flow on the $\beta$-plane as described by Huppert (1975) for an axisymmetric obstacle, because 2.14 reduces to a one-dimensional constraint. However, for eastward flow on the $\beta$-plane, this non-linearly constrained maximum is difficult to find. The search for the critical height is equivalent to looking for a saddle point in the streamfunction. Part of the problem lies in the fact that there can be regions where the total east-west velocity goes to zero in the stationary Rossby waves that exist downstream of the topography, but the north-south velocity does not.

Figure 2.9 shows the critical height as a function of $b$ when closed streamlines first appear at $r=1$. For small enough $b$, this critical height is the global critical height, however, when $b^{1 / 2}$ becomes close to the first zero of $J_{1}$, the resonant solution discussed by McCartney (1975) are valid. The resonant solution first occurs at when $h_{0} / \epsilon=2.65$ and $b=14.86$. In addition, closed streamlines can appear downstream of the topography before they appear over the topography. The critical height that we calculate increases with increasing $b$, and goes to infinity at the resonance point $b=14.86$. The location of the critical stagnation point moves from $\theta=3 \pi / 2$ clockwise 
along the boundary of the topography, until the first resonance is reached (Figure 2.10). The global critical height for closed streamlines to first appear downstream cannot be found easily because the critical stagnation point cannot be constrained.

Examples of consistent frictional solutions where closed streamlines have not formed downstream of the topography can be found. The size of the Taylor column decreases with increasing $b$. It becomes slightly elongated in the north-south direction (Figure 2.11) because the effect of the topography and the planetary vorticity on the circulation is in the same sense so that fluid parcels must travel further to the north, like Johnson's (1983) solution for negative shear. The stagnation point is located on the edge of the topography. The stream function can be calculated according to the integral equation 2.10. The Taylor column reduces the amplitude of the downstream waves, and the effect is felt an order one distance from the topography. The Rossby wave amplitude is smaller in the frictional solution than in the inertial solutions but remains a dominant feature (Figure 2.12). In the inertial solution, the flow is enhanced over the northeast portion of the topography. This flow is reduced in the frictional solution. Once again, the boundary of the Taylor column does not coincide with a streamline of the inviscid solution, although the stagnation point is located at $r=1$ which helps in the search for a solution. The location of the stagnation point of the frictional solution does not coincide with the location of the stagnation point of the stream function at the critical height for the same value of $b$.

The effect of the formation of the Taylor column on the downstream wave-field can be quantified by calculating the wave drag. The wave drag is given by an integral of the pressure around the topography;

$$
h_{0} \oint \psi \cos \theta d \theta \text {. }
$$

The drag for the solutions found in Figure 2.11 are shown in Figure 2.13. The drag is slightly larger than would have been calculated from the inertial solution alone. As $b$ gets larger, the Taylor column is smaller, so that the contribution from $\phi$ to 


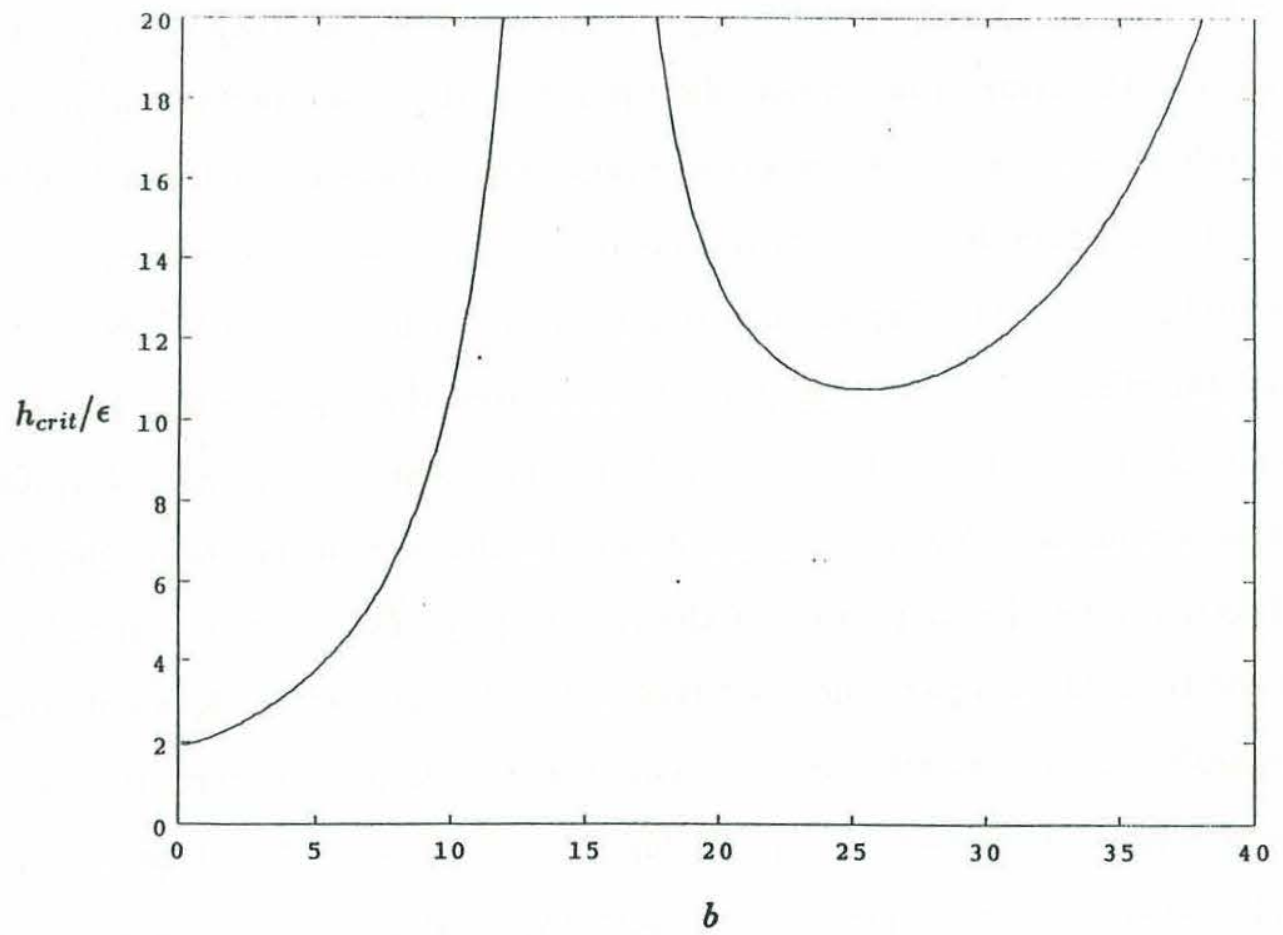

Figure 2.9: Critical height for closed streamlines to form over the topography (at $r=1$ ) for eastward flow over a right circular cylinder on the $\beta$-plane as a function of $b$. In in the $\mathrm{f}$-plane limit, $(b=0)$ the critical height is 2 . The critical height goes to infinity at resonance points of the solution when $b=14.86$ and $b=49.1$. 


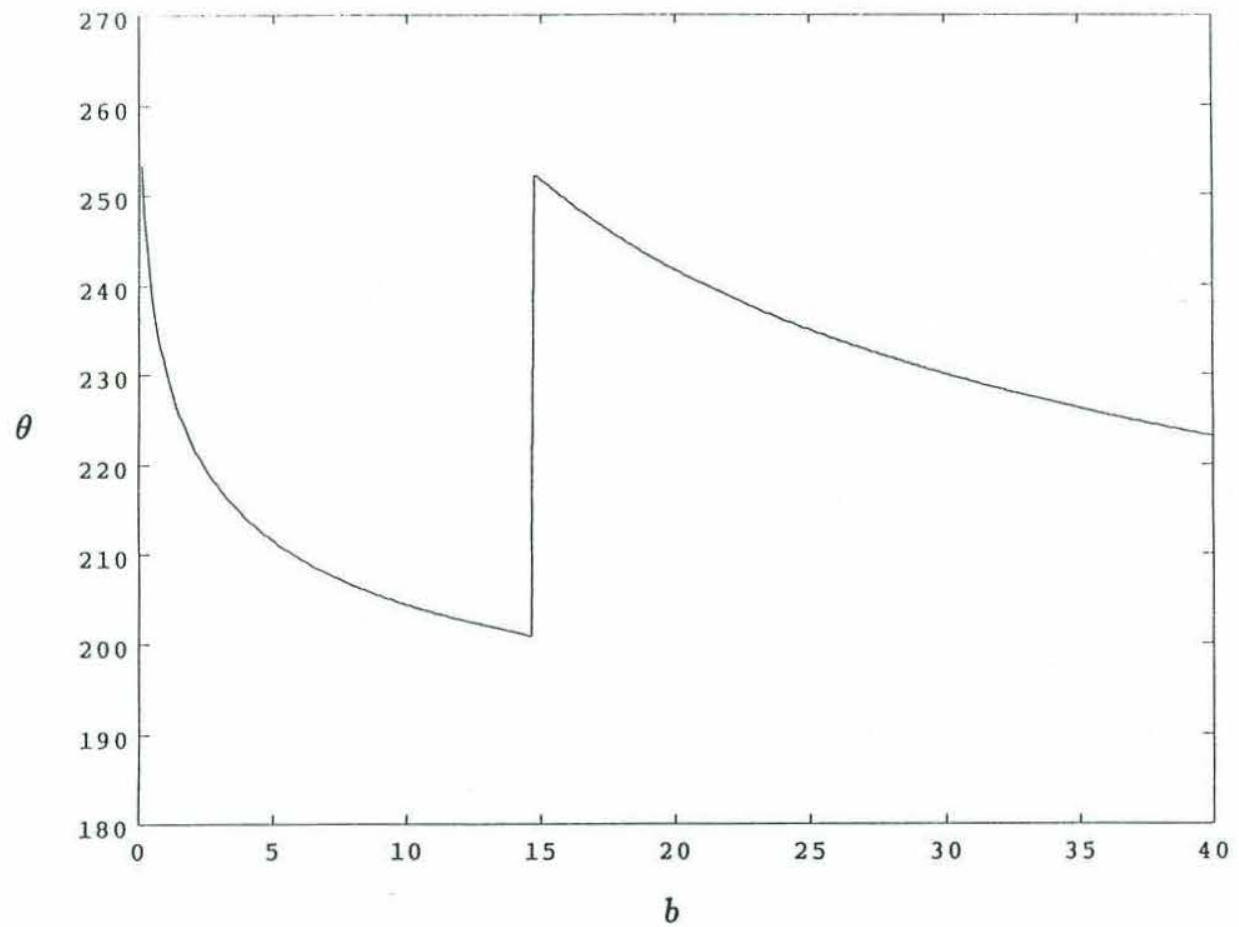

Figure 2.10: The location of the stagnation point in terms of the angle along the boundary of the topography (at $r=1$ ). The stagnation point migrates clockwise as $b$ increases, and then falls back to almost $\theta=3 \pi / 2$ (270 degrees) at the first resonance point. 


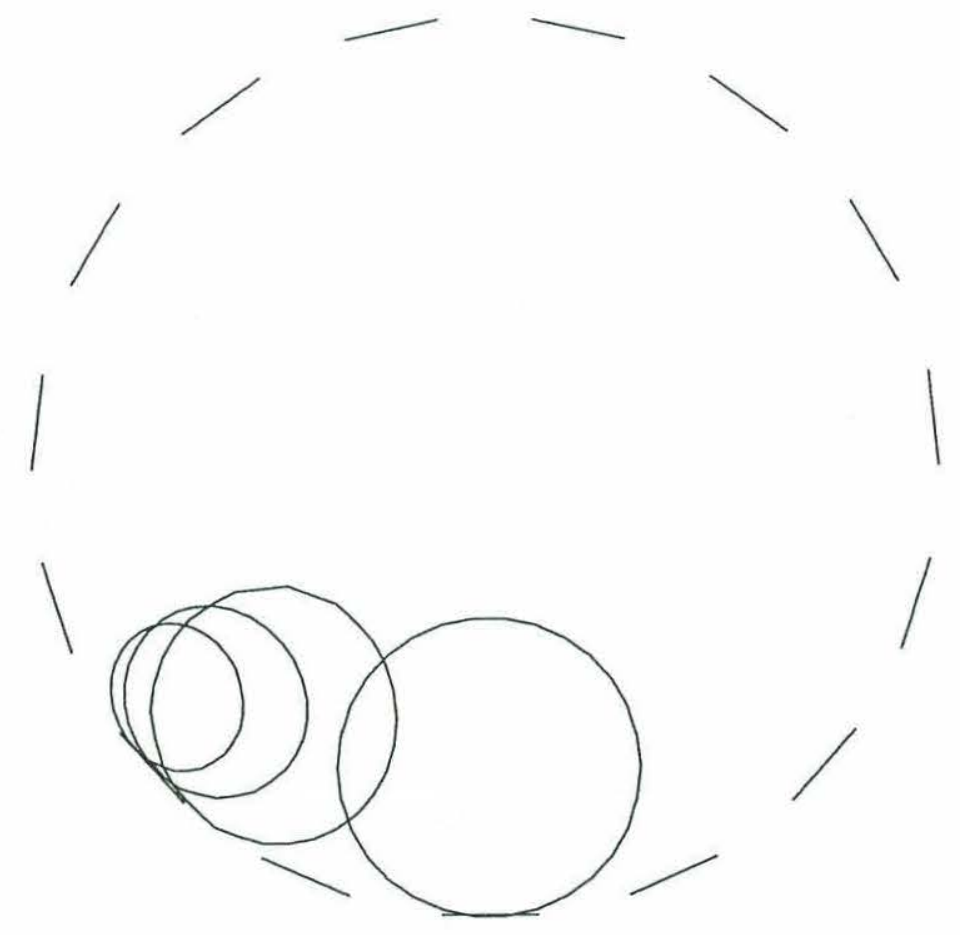

Figure 2.11: Boundary of the Taylor column when $h_{0} / \epsilon=3$ and $b$ is $0,0.5,1$, and 1.5 . Flow is eastward, from left to right. The largest Taylor column has $b=0$, while the smallest has $b=1.5$. The Taylor column is tangent to the edge of the topography, and becomes elongated in $y$ for increasing $b$. Its location is further to the northwest for increasing $b$. 


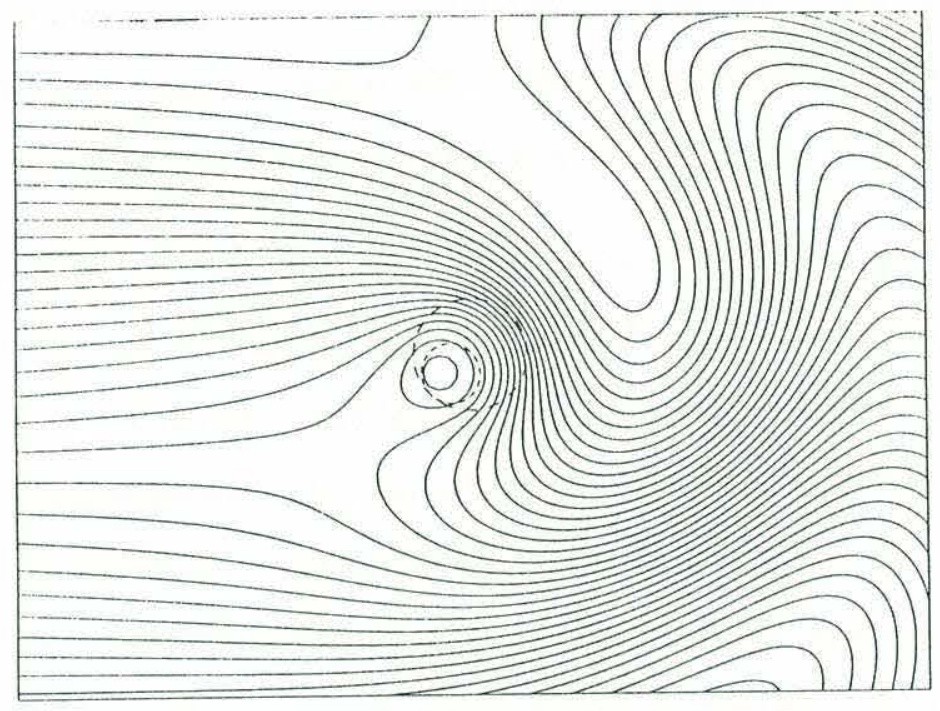

(a)

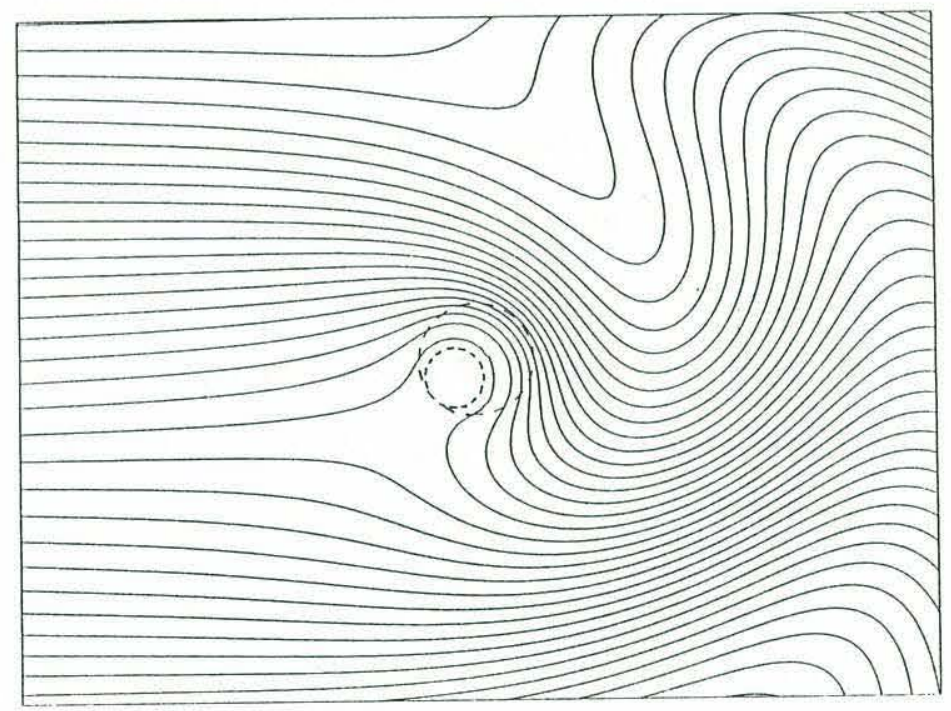

(b)

Figure 2.12: Streamlines for eastward flow over a right circular cylinder of radius unity (dashed), $h_{0} / \epsilon=5$ and $b=0.5$. The streamline contour interval is 0.4 , and the flow is from left to right. (a) The inertial solution $\psi^{(a)}$, the Taylor column boundary is dashed and is not a streamline of the inertial solution. (b) The full solution. The effect of the Taylor column is felt downstream of the topography, and there are no closed streamlines downstream of the topography. 
the drag becomes smaller. We have chosen to display only solutions in which closed streamlines have not formed downstream. This requires that the amplitude of the downstream wave field is small, resulting in less of a contribution to the drag from $\phi$.

When $b$ and $h_{0}$ are large enough, even though the fluid over the topography has been spun down, there are still closed streamlines down stream of the topography. In addition, the first closed streamlines do not always appear over the topography (Figure 2.14). Closed streamlines over the topography are less likely to form as $b$ increases. On the other hand, closed streamlines downstream are more likely to form for increasing $b$. Therefore, there is a finite range of parameters for which one expects to obtain Taylor column solutions in which trapped stagnant fluid is located only over the topography, and there are no other closed streamlines in the solution.

It is important to remember that just because it is possible to find a steadystate solution, it does not mean that it could be realized as a solution to an initial value problem, or that the solution is stable. It has been suggested that when these streamlines form, the flow becomes unstable via barotropic instability by the same mechanism that Lorenz (1972) considered in his study of the instability of interacting Rossby waves. An attempt was made to find a solution where the fluid within the downstream closed streamlines was spun down, but the solution did not converge. It is difficult to determine the appropriate boundary when none of the points of the boundary can be constrained beforehand (this is the same problem that we had when we looked for solutions for non-compact topography on the f-plane). However, this failure could also indicate that solutions of this type are not possible.

\subsection{Summary and conclusions}

The solutions of spun down Taylor columns over several different topographic shapes on the $\mathrm{f}$-plane and over a right circular cylinder on the $\beta$-plane are constructed using 


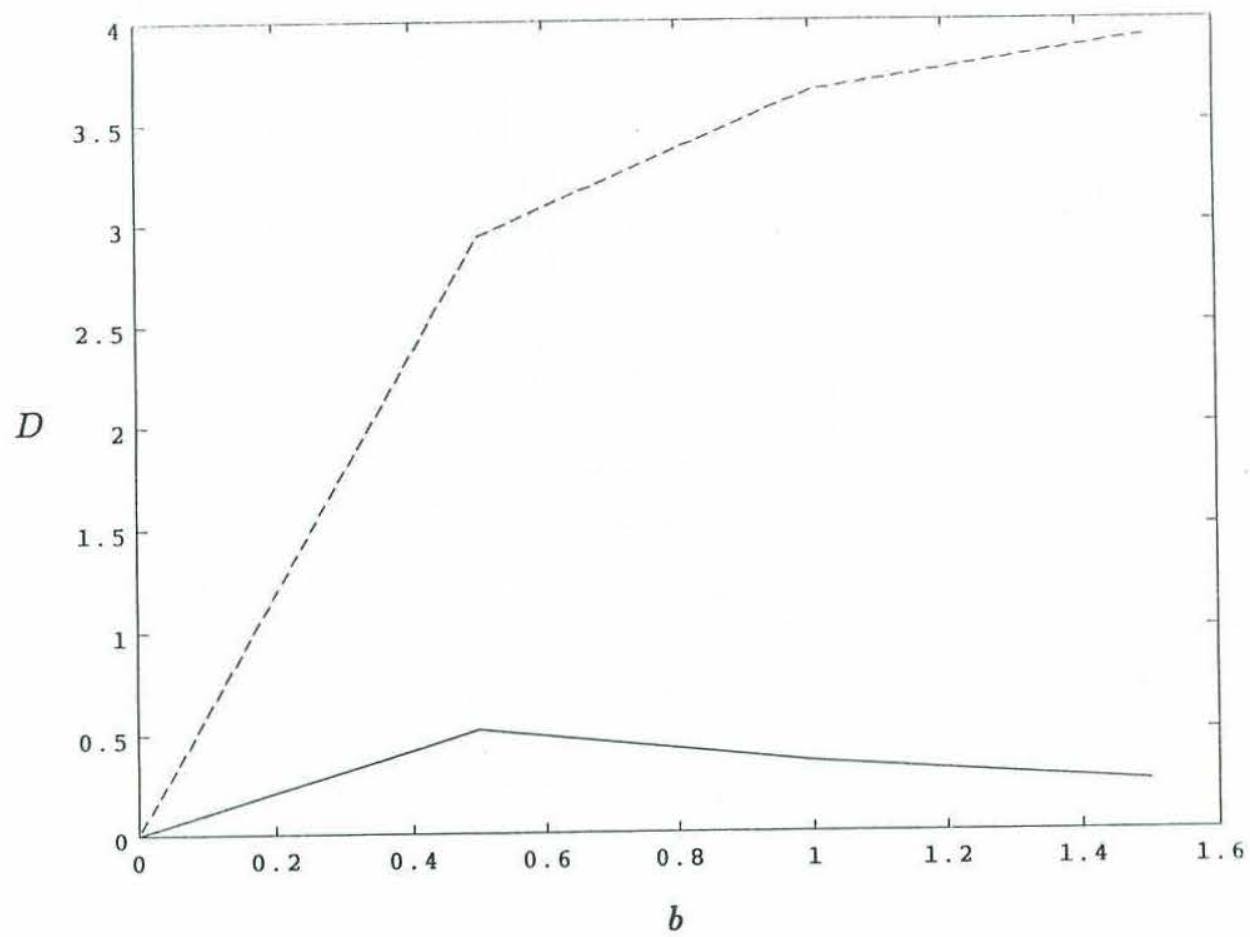

Figure 2.13: Wave drag for the Taylor column solutions found in Figure 2.11. The dashed line is the contribution from the inertial solution. The solid line is the contribution from $\phi$. 


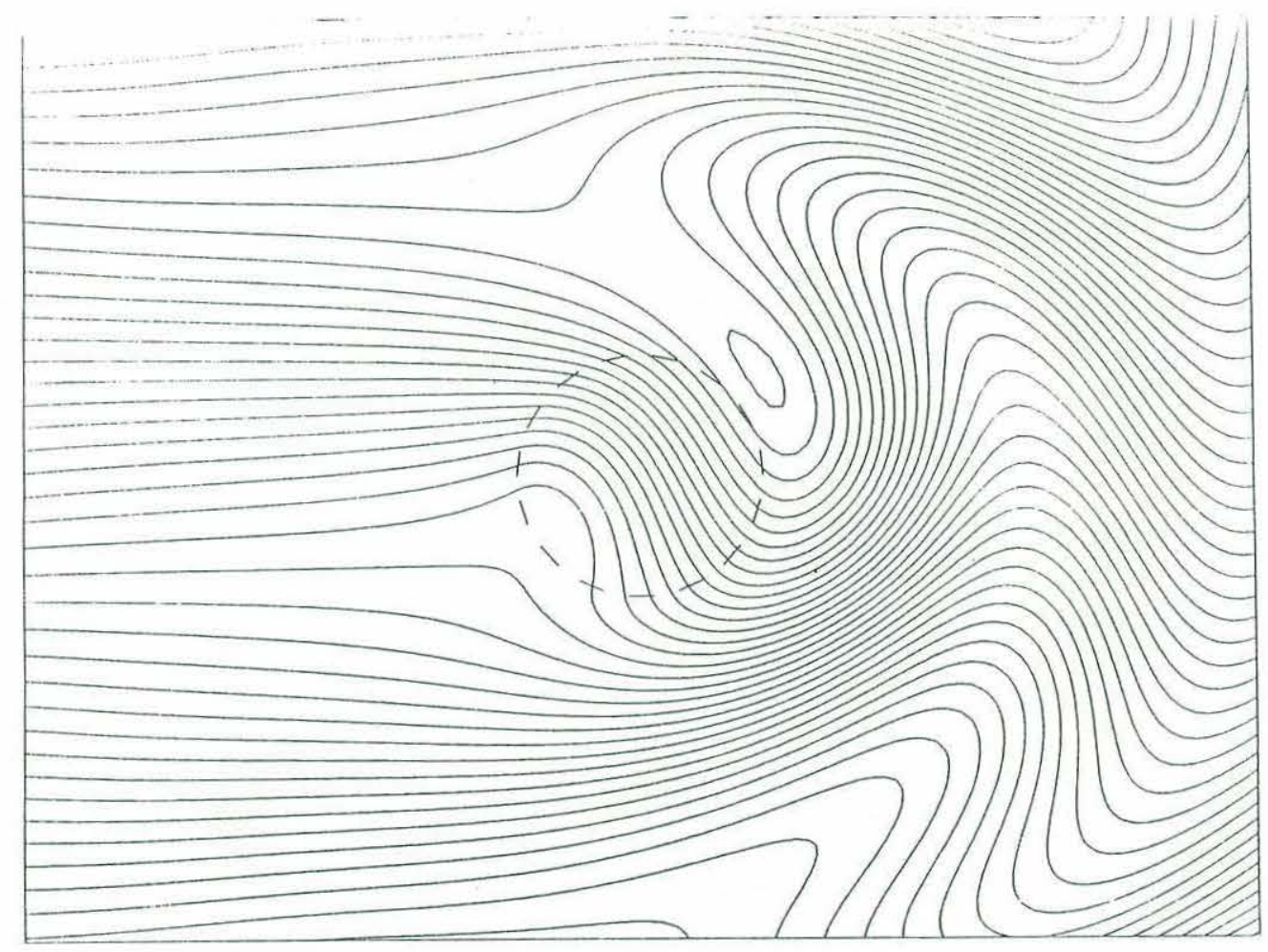

Figure 2.14: Streamlines for the inertial solution $\psi^{(a)}$ for eastward flow over a right circular cylinder of radius unity (dashed), $h_{0} \epsilon=3$, and $b=3$. The streamline contour interval is 0.4 , and the flow is going from left to right. There are closed streamlines downstream of the topography, but not over the topography. 
a boundary integral technique. This technique works well for finding the solutions as long as the initial guess for the correct solution is good and an a priori constraint on the location of the Taylor column can be obtained. For all the solutions found here, the stagnation point of the Taylor column is located on the boundary of the topography. Once the Taylor column boundary is known, the streamline pattern can be calculated. It is found that flow over a right circular cylinder on the $f$-plane is special because the boundary of the Taylor column is a streamline of the inertial solution. This allows an analytic solution to the problem, but is not true for more general topographic shapes or for flow on the $\beta$-plane. It is plausible that all of these Ingersoll type solutions are also maximum retention solutions, where the Taylor column contains fluid that originated over the topography in an initial value problem. In general, the solution to this problem for an intermediate value of $C$ is a valid solution on a time scale short compared with $\tau$, and in this solution $\partial D$ is contained within closed streamlines.

On the f-plane for flow over a cone the Taylor column is elongated in the $y$ direction, and has a tear drop shape. One may expect this general shape for a Taylor column over other topographic shapes with heights that monotonically decrease from $r=0$ and have compact support. It is possible for smooth topography that the friction has order one importance near the stagnation point, and its effect can no longer be considered to be small everywhere, and the formulation of the problem must be altered.

The Taylor column tends to be elongated in the $x$ direction for westward flows on the $\beta$-plane. For eastward flows, the Taylor columns remain more nearly circular, but are slightly extended in $y$ and are moved to the northwest portion of the topography. However, the solution is valid only for relatively small topography and small values of $b$; otherwise, closed streamlines appear downstream of the topography.

All of the solutions that we found in this chapter shared the characteristic that the stagnation point of the frictional solution was located at $r=1$, the boundary of 
the topography. We argue that because it is only possible to construct a solution near the stagnation point with Taylor series expansion when the topography goes to zero at the stagnation point, the problem that Ingersoll (1969) posed can only be solved for topography which vanishes at a finite $r$. However, the Taylor series argument is not a proof of this. In order to investigate the existence of the solution further, an attempt was made to find the solution numerically for flow over topography which falls off like $K_{0}(r)$ for $r>1$. This attempt failed. In addition, it was not possible to find the solution for flow over a cone when the stagnation point of the inertial solution was located at $r<1$. These two failures lead us to conclude that it is not possible to find a solutions for Ingersoll's (1969) problem when the topography is smooth (analytic) everywhere. However, this conclusion is unsatisfactory, because we know that solutions to flow over smooth topography exist when friction is finite. This leads us to conclude that the formulation of the problem must be incorrect when the topography is smooth. Because of this, we anticipate that friction must be of order one importance near the stagnation point, changing the boundary conditions at $\partial D$ and the formulation of the problem. To find out what the solution would look like for flow over smooth topgraphy, one would like to be able to solve for the flow field for flow over smooth topography (say a Guassian bump) and look at solutions in which the bottom friction is successively decreased. Presumeably, examination of the detailed dynamics in such an experiment would indicate appropriate approximations to explore the limit of weak friction. However, the numerical difficulties in calculating the flow for appropriately small frictions may be substantial.

The comparison of the solutions presented here with solutions of the initial value problem is limited and is explored further in Chapter 4. James (1980) and Vaziri and Boyer (1971) demonstrated that the steady solution with finite but small friction has qualitative similarities to Ingersoll's (1969) solution. In both of these studies, the topography has finite extent. Verron and LeProvost (1985) solved the initial value problem on the $\beta$-plane. However, they did not run their model long 
enough to reach a steady state. For eastward flow, it was particularly difficult to reach a steady state because their model was done in a channel and the Rossby waves quickly ran into the walls. Bannon (1980) also solved the initial value problem, but the values of friction that he used were quite high, and he does not show pictures of his solutions for flow on the $\beta$-plane. Unlike all of these solutions, our solution is valid over an infinite domain.

There is a general qualitative result that is seen in both the work presented in this chapter as well as the work of Johnson (1983). If we consider Ingersoll's (1969) solution as the basic solution, then the addition of background shear, $\beta$, or variable topography causes a modification in the shape of the circular Taylor column. The topography introduces positive circulation. If the additional effects have the same sign of circulation (i.e. positive shear) then the Taylor column becomes elongated parallel to the incoming flow; likewise, if the sense of the circulation is opposite to that of the topography (negative shear), then the Taylor column becomes elongated perpendicular to the incoming flow. These ideas also apply to flow on the $\beta$-plane. 


\section{Chapter 3}

\section{Steady solutions for flow over finite topography}

\subsection{Introduction}

Because quasi-geostrophic theory breaks down when the topography becomes large, in this chapter we look at solutions to the problem of inviscid flow over finite topography on the $\beta$-plane. Two models are considered. Barotropic flow over finite topography with a rigid lid is discussed first. In this idealized model, the combined effects of $\beta$, finite topography and finite Rossby number are allowed. Because the model is not quasi-geostrophic, ageostrophic effects exist. The differences between this model and the quasi-geostrophic model considered by McCartney (1975) are discussed in detail, including evaluation of the global effects of the topography on the flow. The model is extremely idealized, requiring no depth variations in the horizontal velocity, and the topography has the simple form of a right circular cylinder. The vertical velocity is limited to the region where topography is varying and is zero everywhere else. However, the physical limitations of the model do not restrict its usefulness for understanding the qualitative structure of flow over finite topography.

The limitations of the barotropic model lead us to consider a second model which allows both finite topography and depth variations in the velocity. We consider 
the simplest model that includes the effect of stratification: the two-layer model. Unfortunately, two-layer shallow-water models do not allow easy calculations of the flow field because the interface height non-linearly couples the two layers. Therefore, we consider a quasi-geostrophic model. In this two-layer model, the topography goes all of the way through the lower layer and extends partly into the upper layer so that quasi-geostrophic theory applies consistently in both layers and the fluid is always quasi-horizontal. This allows the consideration of finite depth variations in a stratified model within physically realizable parameter regimes and allows an analytical solution. This model is restricted in usefulness because of the quasi-geostrophic requirement that the topography extends only an order Rossby number amount into the upper layer and that the flow speeds be small. However, it allows analytic exploration of a new parameter regime in which isopycnals intersect topography, in contrast to existing simple steady models reviewed below. The idea of allowing the lower layer depth to be slightly less than the height of the topography may have wider oceanographic applications than the simple situation discussed here, and this model illustrates what some restrictions of its further use might be. When closed streamlines form the solutions that we discuss in this chapter can only be valid for times short compared to the spin up time $\tau$ and do not satisfy Ingersoll's (1969) critierion for closed streamlines.

\subsection{Background}

In this section, we briefly review models of flow over finite topography in both barotropic and stratified fluids. The literature is limited because of the difficulty of solving the problem analytically.

Johnson (1980) found the solution for flow over finite topography over a right circular cylinder on the f-plane and he showed that when the Rossby number is 
greater than $1 / 4$, closed streamlines will never form. Bannon (1980) considered flow in a barotropic fluid over a Gaussian obstacle. In this study, he investigated the effects of friction, the free-surface, finite Rossby number and non-axisymmetric obstacles. He also briefly discusses the effects of $\beta$. The presence of friction produces an upstream-downstream asymmetry in the flow, while the free-surface allows fluid to go more easily over the topography. Because each solution had to be found numerically, there was limited discussion on the parameter dependence of the solution and global quantities such as the amount of fluid blocked by the topography. He calculated the streamlines for flow on the $\beta$-plane but showed no pictures of the flow field.

There have been several studies of stratified flow over finite topography using both quasi-geostrophic theory and the geostrophic momentum approximation all on the f-plane. In most cases however, these studies have been limited to models in which the bottom is an isopycnal surface, not allowing an intersection of isopycnals with the topography.

Merkine and Kalnay-Rivas (1976) considered stratified flow over Gaussian topography using the geostrophic momentum approximation in a fluid with constant stratification. This approximation allows some ageostrophic effects. The model is inviscid, steady and on the f-plane. As in most steady inviscid models, fluid parcels which end up over the topography are assumed to have originated upstream, even when closed streamlines form. The solution when the background velocity is zero is a fundamental vortex solution, independent of the upstream velocity. The vortex is baroclinic in the near field and barotropic in the far field. Their solution is only valid as long as the bottom boundary remains a constant potential temperature surface, limiting the solution to weak stratification or small topography. However, they note that increasing the stratification results in more fluid parcels going around instead of over the obstacle. There is also an interaction between the topographic vortex and the background flow which is not present in quasi-geostrophic theory, resulting 
in asymmetry in the flow field and some of the fluid is allowed to be split by the topography.

Buzzi and Speranza (1979) discussed flow past finite amplitude obstacles in a stratified fluid using the quasi-geostrophic approximation. They consider both flow over a hemisphere with isentropic lower boundary condition, and flow over a half disk across the basic current with different boundary conditions. The results of the first case are similar to the Merkine and Kalnay-Rivas (1976) work. The results of Buzzi and Speranza's (1979) work showed that the amplitude of the disturbance is proportional to the volume of the axisymmetric obstacle as long as the bottom remains isentropic. In this case the flow falls off as $1 / r$ far away from the obstacle. This is also seen in a model by McCartney (1975) who considered a two-layer model over quasi-geostrophic topography and by Huppert (1975). In contrast, the second case Buzzi and Speranza (1979) considered was a obstacle of small volume but finite vertical cross section, since it was a vertical plate of infinitesimal width. They showed that the condition of impenetrability of a vertical wall reduces to the requirement that the streamfunction depend only on the vertical coordinate along the wall. This case allows flow to go around the obstacle, without actually forming closed streamlines. This flow component decays as $1 / r^{2}$, so that far from the obstacle, the dominant flow is the anticyclonic perturbation.

In order to further understand the effect of the bottom boundary condition on the flow, Schar and Davies (1988) discussed the effect of applying the boundary condition at $z=0$ instead of at $z=h$. Once again the model has an isentropic lower boundary condition. The two boundary conditions give different results. They quantitatively considered the breakdown of the model. When the stratification is strong enough the model breaks down because the lower boundary can no longer be an isopycnal surface. In addition, closed streamlines and a Taylor cap may form when the stratification is weak enough, but these two conditions are distinct. The 
dependence on the stratification for these two breakdown criterion is similar to what has been seen in other quasi-geostrophic models.

\subsection{Flow over finite topography in one layer}

The study of the interaction of finite topography and the effects of $\beta$ have been limited. We show in this section that, in some parameter regimes, this interaction can produce qualitative differences from solutions in which the two effects are considered separately.

Keeping in mind the limitations of theory in which no depth variations in the horizontal velocity are allowed, we consider a barotropic model over finite topography. This model is not quasi-geostrophic and includes both ageostrophic effects and the $\beta$-effect. We consider the flow over a right circular cylinder, allowing an analytic solution and a thorough exploration of the parameters. Since seamounts in the ocean are often tall and steep this study may be qualitatively relevant to the ocean. This model is similar to the model that Bannon (1980) used for his study of flow over gaussian topography. We do not include the free-surface, and this model is inviscid so that analytic progress can be made. The circulation induced by the topography, the force on the topography in both the meridional and zonal directions, the amount of fluid blocked by the topography, and the criterion for closed streamline formation are discussed. Because each of these quantities can also be calculated for the quasigeostrophic model studied by McCartney (1975), a study of the breakdown of the quasi-geostrophic approximation can be made. 


\subsubsection{Model formulation}

We consider inviscid flow in shallow water with a rigid lid on the $\beta$-plane. The momentum equation is given by

$$
\frac{d \mathbf{u}}{d t}+\left(f_{0}+\beta y\right)(\hat{\mathbf{z}} \times \mathbf{u})=\nabla P,
$$

and the continuity equation is

$$
\frac{\partial h}{\partial t}+\nabla \cdot h \mathbf{u}=0
$$

where

$$
\frac{d}{d t}=\frac{\partial}{\partial t}+\mathbf{u} \cdot \nabla
$$

Here, $\mathbf{u}$ is the horizontal velocity, $h$ is the depth of the fluid, $f_{0}$ is the Coriolis parameter, and $\beta$ the latitudinal derivative of the Coriolis parameter. The vertical variations of the horizontal velocity can be ignored as long as the aspect ratio of the fluid is small. We will return to this requirement later.

In steady state both the potential vorticity, $Q$, and the Bernoulli function, $B$, are conserved on streamlines where

$$
Q=\frac{v_{x}-u_{y}+f_{0}+\beta y}{h}
$$

and

$$
B=\frac{u^{2}}{2}+\frac{v^{2}}{2}+P
$$

Here, $P$ is the pressure, and the zonal and meridional components of the velocity are $u$ and $v$ respectively. A transport stream function is defined in steady state by

$$
h u=-\psi_{y},
$$

and

$$
h v=\psi_{x} .
$$


Thus $Q$ and $B$ are both functions of $\psi$ in the steady state.

Assuming that there is a uniform zonal flow of size $U$ upstream of the obstacle, the functional relationship between $Q$ and $\psi$ can be found. Upstream of the obstacle

$$
\psi=-U y H
$$

where $H$ is the constant depth of the fluid far away from the bump. Therefore,

$$
Q(\psi)=\frac{f_{0}}{H}-\frac{\beta \psi}{U H^{2}}
$$

describes the potential vorticity as long as the fluid parcels have originated upstream.

The stream function and $x$ and $y$ can be scaled to obtain a non-dimensional potential vorticity equation. If $\psi$ is scaled by $|U| H L$ where $L$ is the characteristic horizontal scale of the bump, and $x$ and $y$ are scaled by $L$, then using 3.2 and 3.4 the non-dimensional $\psi$ is governed by

$$
\nabla \cdot \frac{\nabla \psi}{h}+\frac{b}{V} h \psi=-\frac{1-h}{\epsilon}-b y
$$

where now $\psi, x, y$, and $h$ are non-dimensional. There are three non-dimensional parameters in this equation, $h$, the non-dimensional depth of the topography, $b$, defined by $\beta L^{2} /|U|$, and the Rossby number $\epsilon=f_{0} /|U| L . \quad V$ in these equations is either 1 or -1 depending on the direction of the flow.

Here, the relative vorticity not only depends on $\psi$ but also $h$, allowing horizontal divergence of the flow field. If $h$ is radially symmetric, then the solution to $\psi$ can be divided into an odd part in $y\left(\psi_{o}\right)$ and an even part in $y\left(\psi_{e}\right)$. The even part is proportional to $1 / \epsilon$ and is forced by the first term on the right hand side of 3.5 , while the odd part is independent of $\epsilon$ and forced by the second term on the right hand side of 3.5. For westward and f-plane flow, the stream function is also even in $x$. However, because of the existence of Rossby waves the upstream boundary conditions introduce upstream-downstream asymmetry for eastward flow as discussed below. 
For an isolated obstacle, the upstream flow remains undisturbed

$$
\psi \rightarrow-V y
$$

for westward and f-plane flow and

$$
r^{\frac{1}{2}}(\psi+y) \rightarrow 0 \quad \frac{\pi}{2}<\theta<\frac{3 \pi}{2}
$$

for eastward flow (McCartney, 1975). This second boundary condition comes from allowing no upstream energy propagation of Rossby waves.

In the quasi-geostrophic case, the $Q, \psi$ relationship is given by 3.4 , but 3.5 becomes

$$
\nabla^{2} \psi+\frac{b}{V} \psi=-\frac{1-h}{\epsilon}-b y .
$$

Notice that the size of the topography only appears on the right hand side, unlike in 3.5. This simpler problem was solved by McCartney (1975) for flow over a right circular cylinder. We assumed that the upstream boundary conditions remain unchanged, but the matching conditions are that $\psi$ and $\psi_{r}$ are continuous across the boundary of the topography. The solution can be written as

$$
\psi=-V y+\phi
$$

where $\phi$ is symmetric. The only antisymmetric part is $-V y$. The pressure is equal to $\psi$ which is continuous at $r=1$. The solution has no horizontal divergence.

The relationship between the solution of flow over tall topography and flow around an obstacle that reaches to the surface should be mentioned. White (1971), and McKee (1971), found the solution for flow around an island on the $\beta$-plane, but, the island problem is not uniquely specified by the formulation given above. The circulation around the island must be given to fully solve the problem. Normally, the circulation is taken to be zero around the island by the following argument; if the flow starts from rest, and circulation is never introduced into the system, then 
circulation will never develop. The stream function around the island as stated above is completely odd in $y\left(\psi_{e}=0\right)$. As will be seen, this choice of circulation is not the limit of a bump going to the surface. The two solutions are different because there is always vortex stretching when flow goes over a bump and circulation must be induced, while vorticity is never produced when inviscid flow interacts with an island.

To be able to solve the problem analytically, flow over a right circular cylindrical bump (Figure 1.1b) is investigated where

$$
h= \begin{cases}h_{0} & r<1 \\ 0 & r>1 .\end{cases}
$$

To solve for the stream function, two matching conditions are needed at $r=1$. First, by integrating the continuity equation 3.1 across $r=1$, it can be seen that the mass transport, $\psi$, must be continuous. We allow the tangential velocity, $\psi_{r} / h$, to be continuous also. This allows the vorticity to be finite at $r=1$. This condition requires that the pressure be discontinuous at $r=1$. The requirement that the pressure is discontinuous at the boundary of the topography is discussed further in Appendix B where we consider the solution of flow over linearly changing topography and show that in the limit of a finite step, the matching conditions reduce to those that we choose here.

The functional relationship between $B$ and $\psi$ can be found from the upstream boundary condition which gives

$$
B(\psi)=\psi-\frac{V \epsilon b \psi^{2}}{2}
$$

The first term is the geostrophic part of the pressure, which is the only contribution in quasi-geostrophy. The second term results from the inclusion of $\beta$ in the finite depth model and being proportional to $\epsilon$, would vanish in the quasi-geostrophic limit. Using 3.3 and 3.8 , the pressure is then

$$
P=\psi-V \epsilon \beta \frac{\psi^{2}}{2}-\frac{\epsilon}{2 h^{2}}\left({\psi_{r}}^{2}+\psi_{\theta}{ }^{2}\right) .
$$


This topography allows analytic solution of the problem. However, it requires violation of one of the requirements of the shallow water model, that is that the advection by the vertical velocity is small. In fact, under the shallow water equations, the vertical velocity is given by

$$
w=-z\left(u_{x}+v_{y}\right)
$$

From 3.10 the vertical velocity at $r=1$ is proportional to a delta function since the radial velocity changes discontinuously. In order to reconcile this we reinterpret the model as being an approximation to smooth topography, where the scale of the variation of the topography is small compared to the relevant length scale of the problem (i.e. the radius of the topography), but is still much greater than the depth of the fluid. This is the same condition that we require in Appendix B to reconcile having discontinuous pressure.

\subsubsection{Calculation of quantities derived from $\psi$}

In order to analyze the solutions to 3.5 , several quantities of physical interest are calculated, and the results for these calculations are compared to the results found using the quasi-geostrophic approximation. These results give insight as to when the quasi-geostrophic approximation can be used and what sort of errors result from its use. The flow can be influenced in several ways by the topography. Circulation is induced by the topography, the flow can be blocked by the topography, and the flow exerts a force on the topography. These quantities can all be calculated with knowledge of the stream function.

The flow structure is influenced by the circulation which is induced over the topography due to fluid parcels that originate upstream being moved over the topography. The magnitude of the circulation $\Gamma_{c}$ can be calculated in the usual way by 
doing a line integral about the topography.

$$
\Gamma_{c}=\frac{1}{h} \oint \psi_{r} d \theta
$$

Since only the symmetric part of the solution contributes to this integral, $\Gamma_{c}$ is proportional to $1 / \epsilon$ as Bannon (1980) also pointed out. This result holds for quasigeostrophic flow also.

The amount of fluid that goes over the topography relative to the undisturbed flow upstream of the topography is the blocking efficiency. This quantity is defined by

$$
T(y)=1-\frac{\psi(y)-\psi(-y)}{2 V y} .
$$

To understand this we consider each of the parts of 3.12 separately. $2 V y$ is the transport approaching the obstacle between $y$ and $-y$ at $x=0 . \psi(y)-\psi(-y)$ is the transport between $y$ and $-y$. So $1-T$ is the fraction of transport passing over the bump, and finally $T$ is the fraction of transport diverted around the bump. Because quasi-geostrophic theory does not allow horizontal divergence of the fluid, none of the fluid is diverted around the obstacle, and $T=0$. Note that $T(\infty)=0$. When $T>0$ the flow is blocked, and when $T<0$ the transport is enhanced. Also, $T$ depends only on $\psi_{o}$, the odd portion of the stream function, and is therefore independent of $\epsilon$. Bannon (1980) and Johnson (1978) pointed this out for f-plane flows, but it is also true for flows on the $\beta$-plane, when the topography is radially symmetric. The calculation of $T$ does not take into account closed streamlines, which would alter our notion of blocking efficiency. In the calculations made, we ignore this effect and evaluate $T$ at $y=1$.

The flow exerts a force on the topography, or conversely, the topography exerts a force on the flow. The force can be divided into the drag, $D$, the force in the zonal direction, and the lift $L$, the force in the meridional direction, which can be thought of as the Coriolis force acting on the topography. The forces on the bump are given 
by

$$
\mathbf{F}=\int P \nabla h d A .
$$

Here, $h$ is discontinuous, $h_{r}=h_{0} \delta(1-r)$, and the pressure is discontinuous. To evaluate this integral, we consider a smooth, steep topography, with $h_{r}$ symmetric about $r=1$, close to a delta function. As the topography becomes steeper, the integral of $P h_{r}$ over $r$ tends toward $h_{0} \frac{1}{2}\left(P\left(r=1^{+}\right)+P\left(r=1^{-}\right)\right)$. This makes sense if we consider the topography to be an approximation to smooth topography. Thus,

$$
D=\oint h_{0} \frac{1}{2}\left(P\left(r=1^{+}\right)+P\left(r=1^{-}\right)\right) \cos \theta d \theta
$$

and

$$
L=\oint h_{0} \frac{1}{2}\left(P\left(r=1^{+}\right)+P\left(r=1^{-}\right)\right) \sin \theta d \theta .
$$

The evaluation of the forces in the quasi-geostrophic model is simpler because the pressure is continuous and is proportional to the stream function. To be consistent, the pressure is evaluated by 3.9 including the quadratic terms for finite topography.

The pressure can be divided into its odd and even parts $P=P_{e}+P_{o}$ where

$$
P_{o}=\psi_{o}-V \epsilon \beta \psi_{e} \psi_{o}-\frac{\epsilon}{h^{2}}\left(\psi_{e_{r}} \psi_{o_{r}}+\psi_{e \theta} \psi_{o \theta}\right)
$$

and

$$
P_{e}=\psi_{e}-V \epsilon \beta \frac{\left(\psi_{e}^{2}+\psi_{o}^{2}\right)}{2}-\frac{\epsilon}{2 h^{2}}\left(\psi_{e_{r}}^{2}+\psi_{o_{r}}^{2}+\psi_{e \theta}^{2}+\psi_{o \theta}^{2}\right) .
$$

$P_{o}$ is independent of $\epsilon$, because $\psi_{e}$ is proportional to $1 / \epsilon$ and $\psi_{o}$ is independent of $\epsilon$. The lift only depends on $P_{o}$, so it too is independent of $\epsilon$. Meanwhile, $P_{e}$ has terms proportional to $\epsilon$ and $1 / \epsilon$ so the drag is a more complicated function of $\epsilon$. For westward flow and flow on the f-plane, the pressure is even about the $y$ axis, so that the drag is zero. 


\subsubsection{Solutions on the $f$-plane}

The results in this section are not new; however, they set a context for the $\beta$-plane results. Ingersoll (1969) found the f-plane solutions using the quasi-geostrophic approximation, while Johnson (1978) found the finite depth solution, which is given by

$$
\psi=-r \sin \theta-\frac{h_{0}}{2 \epsilon} \ln r+\frac{h_{0}}{2-h_{0}} \frac{\sin \theta}{r}
$$

for $r>1$, and

$$
\psi=-\frac{h_{0}}{4 \epsilon}\left(1-h_{0}\right)\left(r^{2}-1\right)-\frac{2-2 h_{0}}{2-h_{0}} r \sin \theta
$$

for $r<1$. The critical height for closed streamlines to form can be found by considering the point where $\psi_{\theta}$ and $\psi_{r}$ are zero. The stagnation point when the height is at the critical height occurs at $\theta=3 \pi / 2$ and $r=1$ for eastward flow. The critical height for closed streamlines to form is

$$
h_{\text {crit }}=1-\sqrt{1-4 \epsilon} .
$$

As Johnson(1978) pointed out, no closed streamlines occur when $\epsilon>1 / 4$ no matter how tall the bump is. When $h_{0} \rightarrow 1$, the solution for $r>1$ becomes

$$
\psi=-\frac{1}{2 \epsilon} \ln r-r \sin \theta+\frac{\sin \theta}{r} .
$$

The circulation is finite $\left(\Gamma_{c}=-\pi / \epsilon\right)$, but is not the value found by taking the limit of Ingersoll's (1969) frictional solution as $h_{0} / \epsilon \rightarrow \infty$ which is $-4 \pi$. The solution becomes the solution for irrotational flow around a cylinder as $\epsilon$ becomes large.

On the f-plane the quasi-geostrophic solution for $\psi$ is

$$
\psi=-r \sin \theta-\frac{h_{0}}{2 \epsilon} \ln r
$$

for $r>1$, and

$$
\psi=-\frac{h_{0}}{4 \epsilon}\left(r^{2}-1\right)-r \sin \theta
$$


for $r<1$. The critical height in quasi-geostrophy is simply the limit of $\epsilon \rightarrow 0$ in $3.15,2 \epsilon$. The quasi-geostrophic solution underestimates the strength of the flow over the topography, which results in an over-estimate of $h_{c r i t}$. The blocking efficiency for the finite depth solution $T$ is $h_{0} /\left(2-h_{0}\right)$ which is always greater than the quasigeostrophic blocking efficiency of zero. This results because the quasi-geostrophic approximation does not allow horizontal divergence of the flow caused by the finite height of the topography.

The circulation induced by the topography is

$$
\Gamma=-\frac{h_{0}}{\epsilon} \pi
$$

for both the QG and the shallow water solutions. The lift is $\pi$ in both formulations.

\subsubsection{Solutions for westward flow on the $\beta$-plane}

When the flow over the topography is to the west and the effects of $\beta$ are included the solution is composed of modified bessel functions $K_{n}$ and $I_{n}$. The introduction of $\beta$ traps the disturbance near the topography. For $r>1$

$$
\psi=r \sin \theta+a_{1} K_{0}\left(b^{1 / 2} r\right)+a_{2} K_{1}\left(b^{1 / 2} r\right) \sin \theta
$$

and for $r<1$

$$
\psi=\frac{r \sin \theta}{\left(1-h_{0}\right)}+b_{1} I_{0}(\kappa r)+b_{2} I_{1}(\kappa r) \sin \theta+F_{0}
$$

where

$$
F_{0}=\frac{h_{0}}{\epsilon b\left(1-h_{0}\right)}
$$

and

$$
\kappa^{2}=b\left(1-h_{0}\right)^{2} .
$$

The coefficients $a_{1}, a_{2}, b_{1}$, and $b_{2}$ are found from the matching conditions at $r=1$, and are not given here. The disturbance is trapped within a distance comparable to 
$1 / b^{1 / 2}$. The quasi-geostrophic approximation gives the solution (McCartney, 1975)

$$
\psi=r \sin \theta+\frac{h_{0}}{\epsilon b^{1 / 2}} \begin{cases}I_{1}\left(b^{1 / 2}\right) K_{0}\left(b^{1 / 2} r\right) & r>1 \\ -K_{1}\left(b^{1 / 2}\right) I_{0}\left(b^{1 / 2} r\right)+\frac{1}{b^{1 / 2}} & r<1 .\end{cases}
$$

We notice here that the quasi-geostrophic and the finite depth solutions are fundamentally different because the odd portion of the solution is not simply the background flow. This was true on the f-plane also.

To decide what the appropriate parameter values to consider, we consider the Coriolis parameter where

$$
f=f_{0}+\beta y^{\prime}=f_{0}(1+b \epsilon y)
$$

where $y^{\prime}$ is dimensional and $y$ is non-dimensional. For the topography to be located totally in the northern hemisphere, we must require that $b \epsilon<1$ so that $f>1$ everywhere. We restrict ourselves to cases in which this is true because we are primarily interested in the effects of mesoscale topography on the flow field, not large scale topography which straddles the equator. The oceanographic applications of this model are probably limited to situations in which $b \epsilon$ is fairly small. In the cases we consider below, we let $h_{0}=.6$ and $b \epsilon=.6$ so that the two effects are comparable and the differences between the quasi-geostrophic solution and the finite depth solution can be illustrated.

When the Rossby number is large, the finite depth solution allows fluid to be partially split by the obstacle so that some fluid goes around the obstacle north of its starting latitude, and the streamlines are spread over the topography. This feature is allowed by the horizontal divergence associated with the finite depth solution and results in blocking of the flow. Thus, the solution is more odd about the $x$ axis, unlike the quasi-geostrophic solution (Figure 3.1). The effect of $\beta$ is to trap the response close to the topography. When the Rossby number is smaller $(\epsilon=.2)$, the difference between the two solutions is not as remarkable, although the spreading of 
the streamlines over the topography is still apparent (Figure 3.2). The symmetry of the finite depth solution is noticeably different in Figure 3.2 than in 3.1.

When $b$ is large, the solution is qualitatively different. In this case, the streamlines tend to follow lines of constant background potential vorticity, and an approximate solution can be found. The relative vorticity in the potential vorticity equation 3.5 can be neglected except in boundary layers at $r=1$. The streamlines follow lines of constant $y$ everywhere, except in the boundary layers. The solution for $r>1$ is then

$$
\psi=r \sin \theta+\frac{h_{0}}{2\left(1-h_{0}\right)} e^{-\xi} \sin \theta+\frac{h_{0}}{2\left(1-h_{0}\right) \epsilon b} e^{-\xi}
$$

while for $r<1$

$$
\psi=\frac{r \sin \theta}{1-h_{0}}-\frac{h_{0}}{2\left(1-h_{0}\right)} e^{\xi\left(1-h_{0}\right)} \sin \theta-\frac{h_{0}}{2\left(1-h_{0}\right) \epsilon b} e^{\xi\left(1-h_{0}\right)}+\frac{h_{0}}{\left(1-h_{0}\right) \epsilon b},
$$

where $\xi=(r-1) b^{1 / 2}$ is the boundary layer variable. The solution is correct to order $1 / b^{1 / 2}$. We have let $b \epsilon$ be order one in this solution while $b$ is large. Thus for this solution, $\epsilon$ is small while $h_{0}$ can be finite. Consider a fluid parcel which originates upstream at a $y$ location $y_{e}$ and has potential vorticity $f_{0}+\beta y$. If we move this parcel over the topography to a $y$ location $y_{i}$, while conserving its potential vorticity, we find that

$$
f_{0}+\beta y_{e}=\frac{f+\beta y_{i}}{1-h_{0}}
$$

Therefore,

$$
y_{i}=y_{e}\left(1-h_{0}\right)-\frac{f_{0} h_{0}}{\beta} \text {. }
$$

Since $y_{e}>0$, fluid parcels always moves to the south over the topography in this limit. The southward movement is larger than would be predicted from quasi-geostrophic theory because of the finite topographic variations and the amount of fluid going over the topography can be enhanced relative to the background flow. In addition, the displacement is stronger in the northern part of the topography because $f$ is larger. 


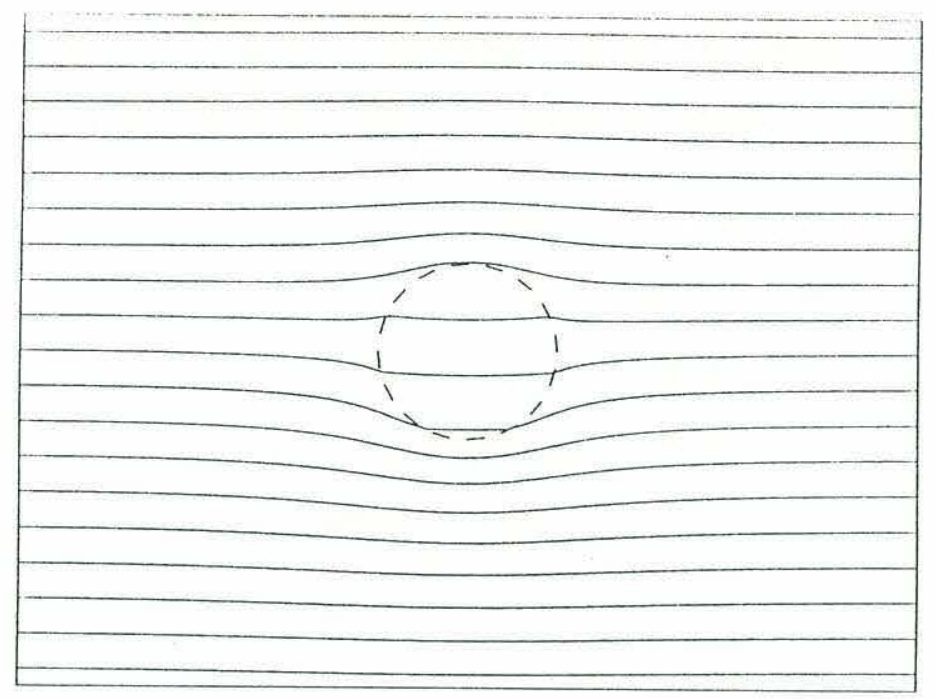

(a)

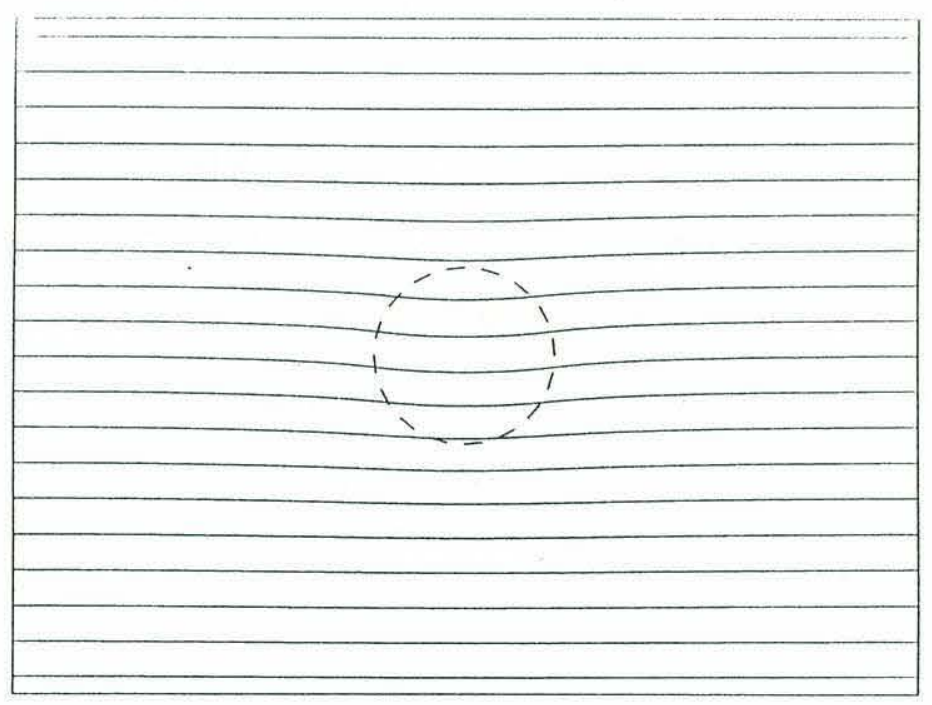

(b)

Figure 3.1: Stream function for westward flow when $b=.3, h_{0}=0.6$, and $\epsilon=2$. In this and all subsequent figures of contours of streamlines the contour interval is 0.4 , and the bump is represented by the circle (dashed) with radius 1 . (a) Finite depth solution, (b) quasi-geostrophic solution. 


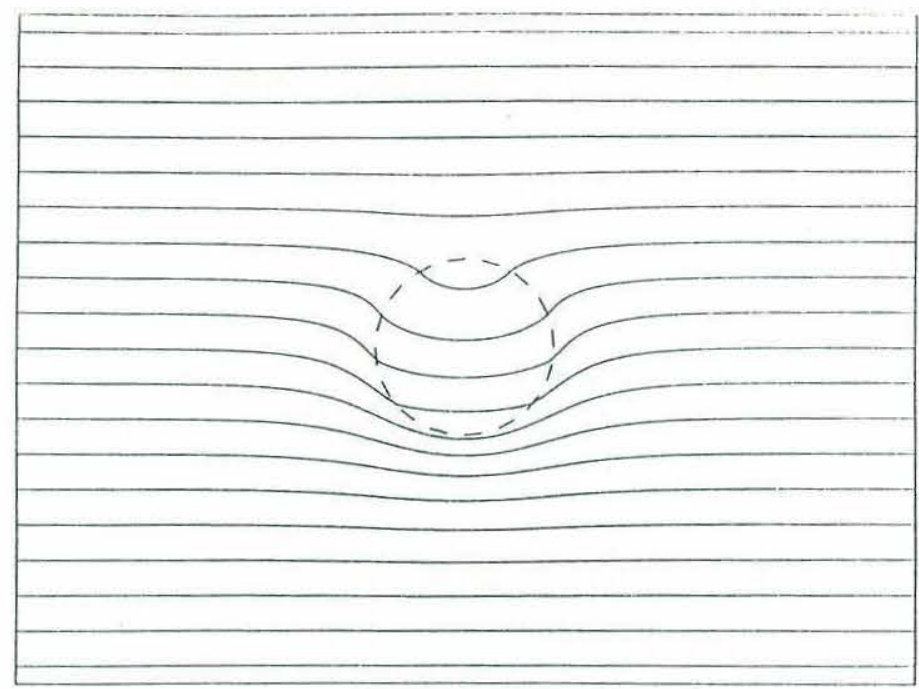

(a)

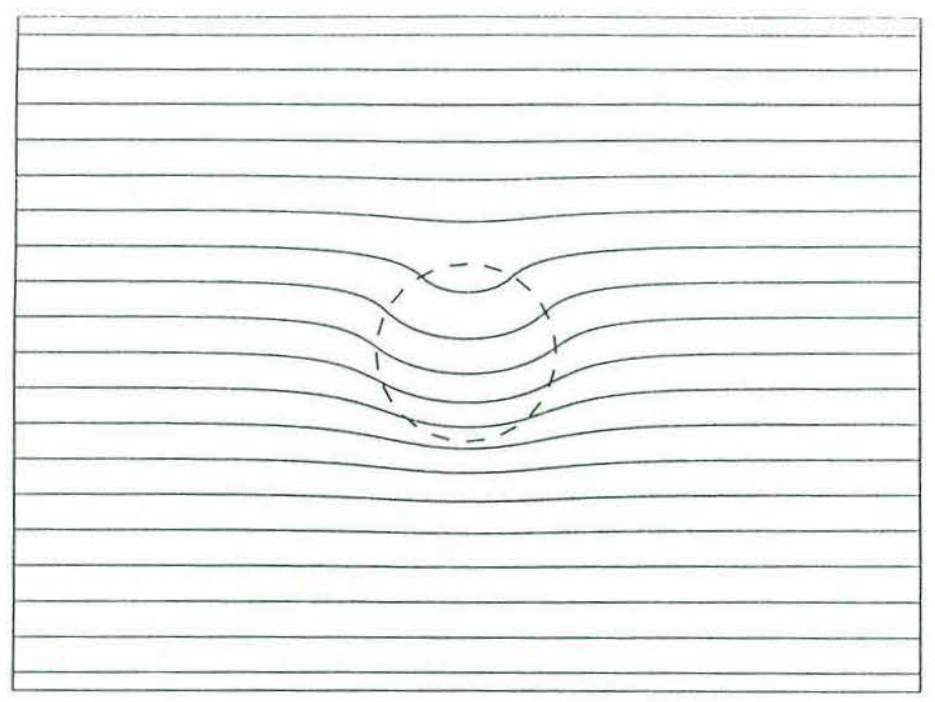

(b)

Figure 3.2: Stream function for westward flow when $b=3, h_{0}=0.6$, and $\epsilon=.2$. (a) Finite depth solution, (b) quasi-geostrophic solution. 
In this limit the quasi-geostrophic solution can be found by simply letting $h_{0}$ go to zero in 3.16 and 3.17 while keeping $h_{0} / \epsilon$ and $b \epsilon$ order one:

$$
\psi=r \sin \theta+\frac{h_{0}}{2 \epsilon b} e^{-\xi}
$$

for $r<1$, and

$$
\psi=r \sin \theta+\frac{h_{0}}{2 \epsilon b} e^{\xi}-\frac{h_{0}}{\epsilon b}
$$

for $r<1$.

From 3.16 and 3.17 , the circulation, lift, and blocking can be calculated when $b$ is large;

$$
\begin{gathered}
L=\pi \frac{\left(2-h_{0}\right)^{2}}{4\left(1-h_{0}\right)^{2}}, \\
\Gamma_{c}=-\frac{\pi h_{0}}{\epsilon b^{1 / 2}\left(1-h_{0}\right)}
\end{gathered}
$$

and

$$
T=-\frac{h_{0}}{2\left(1-h_{0}\right)} .
$$

Note that the large $b$ limit for the quasi-geostrophic solution gives

$$
\Gamma_{c}=-\frac{\pi h_{0}}{\epsilon b^{1 / 2}},
$$

as one would expect from taking the small $h_{0}$ limit of 3.18 . These quantities are all accurate to order $1 / b^{1 / 2}$. The interesting thing about this solution is that $T$ is negative because the flow is enhanced over the topography due to the conservation of total vorticity as described above. An example of the solution when $b$ is large is shown in Figure 3.3. In both the quasi-geostrophic solution and the finite depth solution, the streamlines are deflected to the south, and match to the exterior flow field through the boundary layers. The relative vorticity for the case shown in Figure 3.3 is important because the boundary layers take up almost half of the topography even for this large value of $b$. 


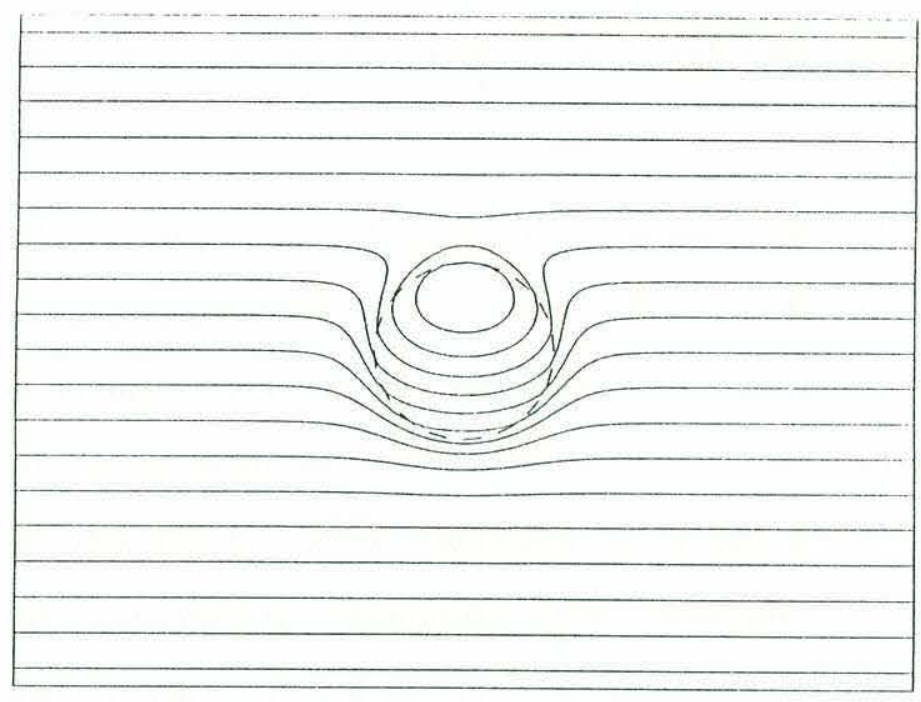

(a)

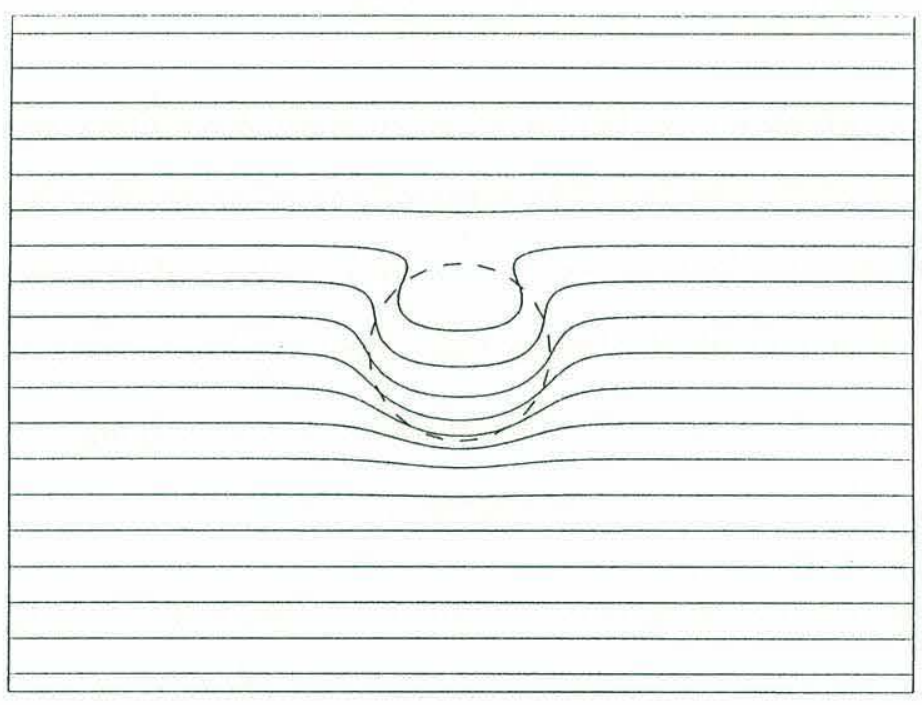

(b)

Figure 3.3: Stream function for westward flow when $b=20, h_{0}=0.6$, and $\epsilon=0.5$. (a) Finite depth solution, (b) quasi-geostrophic solution. 
Having explored the two limits of the $\beta$-plane solution, $\mathrm{f}$-plane flow and large $b$ flow, we return to the full solution and discuss the global quantities that can be calculated from $\psi$.

The quasi-geostrophic circulation and the shallow water circulation are both proportional to $\pi h_{0} / \epsilon$, so that the two solutions can be compared as a function of $b$ and $h_{0}$ only since the dependence on the Rossby number is the same (Figure 3.4). The quasi-geostrophic solution consistently underestimates the effect of the topography. The shallow water solution allows an additional effect on the circulation as $h_{0}$ is increased because the full solution allows horizontal divergence of the streamlines which increases with increasing $h_{0}$. The limit in 3.18 is reached slowly, since the boundary layer solution has corrections which are order $1 / b^{1 / 2}$. The circulation is always smaller than that obtained on the $\mathrm{f}$-plane $(b=0)$, since the $\beta$-effect works to compensate the vortex squashing over the topography.

The lift is always greater than that associated with the $\mathrm{f}$-plane solution $(b=0)$ (Figure 3.5). The effect of $\beta$ is to keep the streamlines more nearly aligned with the lines of constant $\left(f_{0}+\beta y\right) / h$. As $b$ increases, the odd portion of the stream function, $\psi_{o}$, dominates. This effect was illustrated in the boundary layer solution above. The quadratic terms in the pressure also contribute to the lift, rectifying the even part of $\psi$, and contributing to the force on the topography.

When $b$ is small, the blocking is larger for larger $h_{0}$, but is less than the value on the f-plane. Because the $\beta$-effect helps to compensate the vortex squashing caused by fluid parcels rising up on the topography, the fluid tends to go more easily over the topography than be diverted by the topography on the $\beta$-plane (Figure 3.6). However, when $b$ becomes large, the blocking efficiency is negative as we predicted from the boundary layer solution. In this case, the fluid parcels conserve background potential vorticity $\left[\left(f_{0}+\beta y\right) / h\right]$ and the streamlines become close together over the topography, resulting in enhanced transport. This effect can be seen in Figure 3.3. 


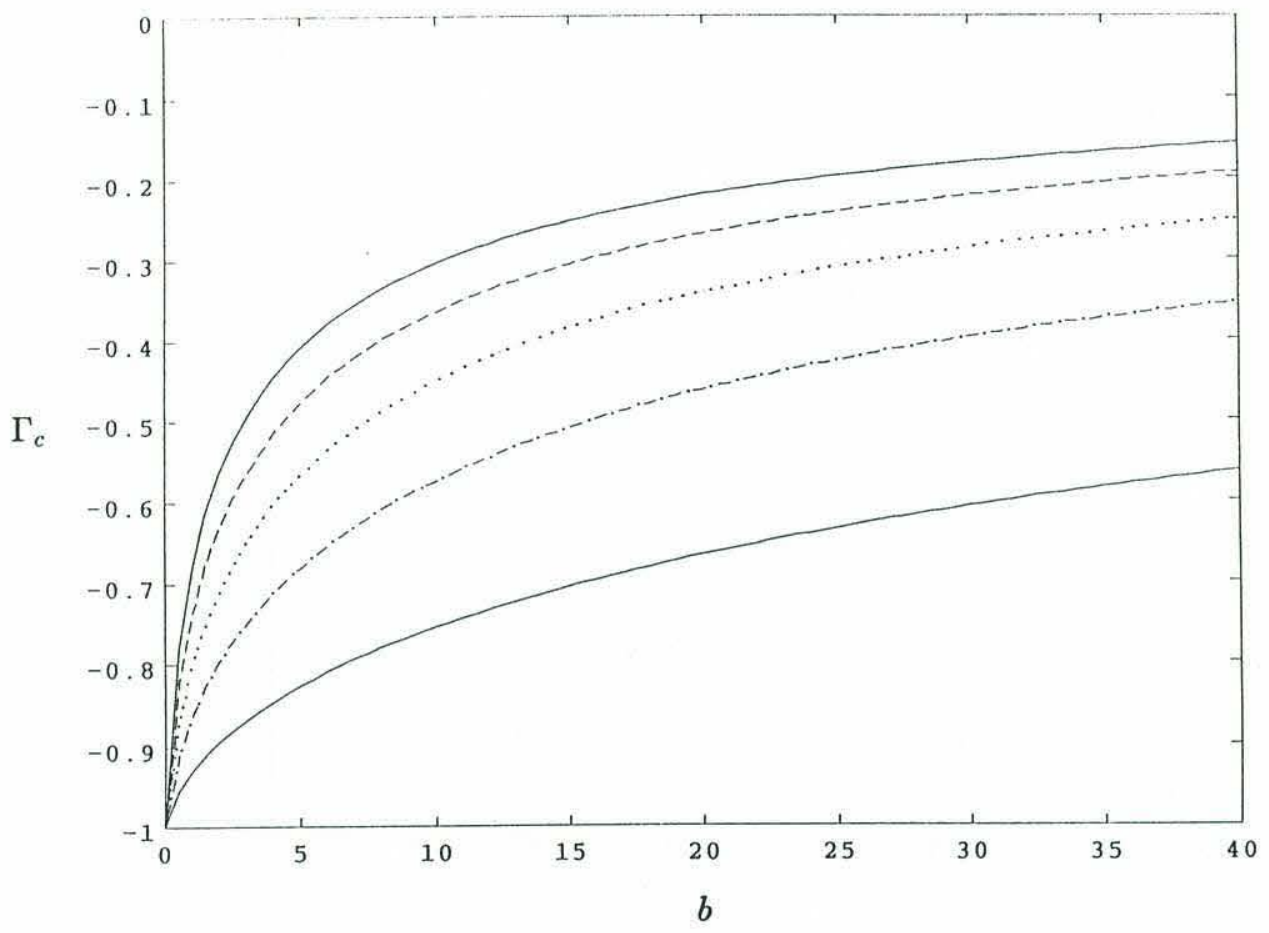

Figure 3.4: The circulation $\Gamma_{c}$ for westward flow. The quasi-geostrophic solution (lower solid line), the shallow water solution for $h_{0}$ of 0.2 (dashed line), 0.4 (dasheddotted line), 0.6 (dotted line) and 0.8 (solid line). It is shown in units of $h_{0} \pi / \epsilon$. The sign of the circulation is always negative (anticyclonic). 


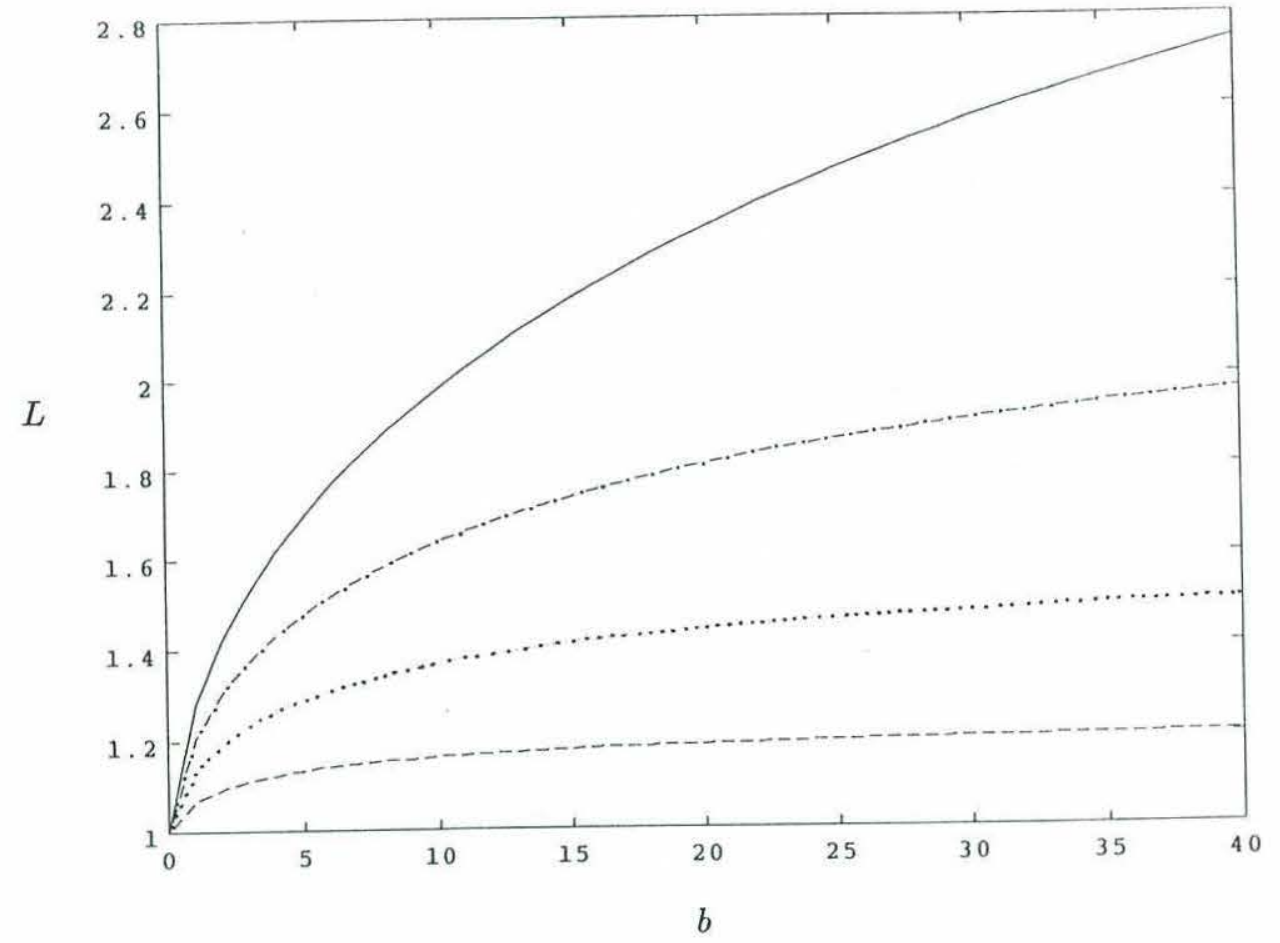

Figure 3.5: Lift $L$ for westward flow for the shallow water solution for $h_{0}$ of 0.2 (dashed line), 0.4 (dotted line), 0.6 (dotted-dashed line) and 0.8 (solid line). It is shown in units of $\pi$ so that the quasi-geostrophic lift has magnitude 1 . 


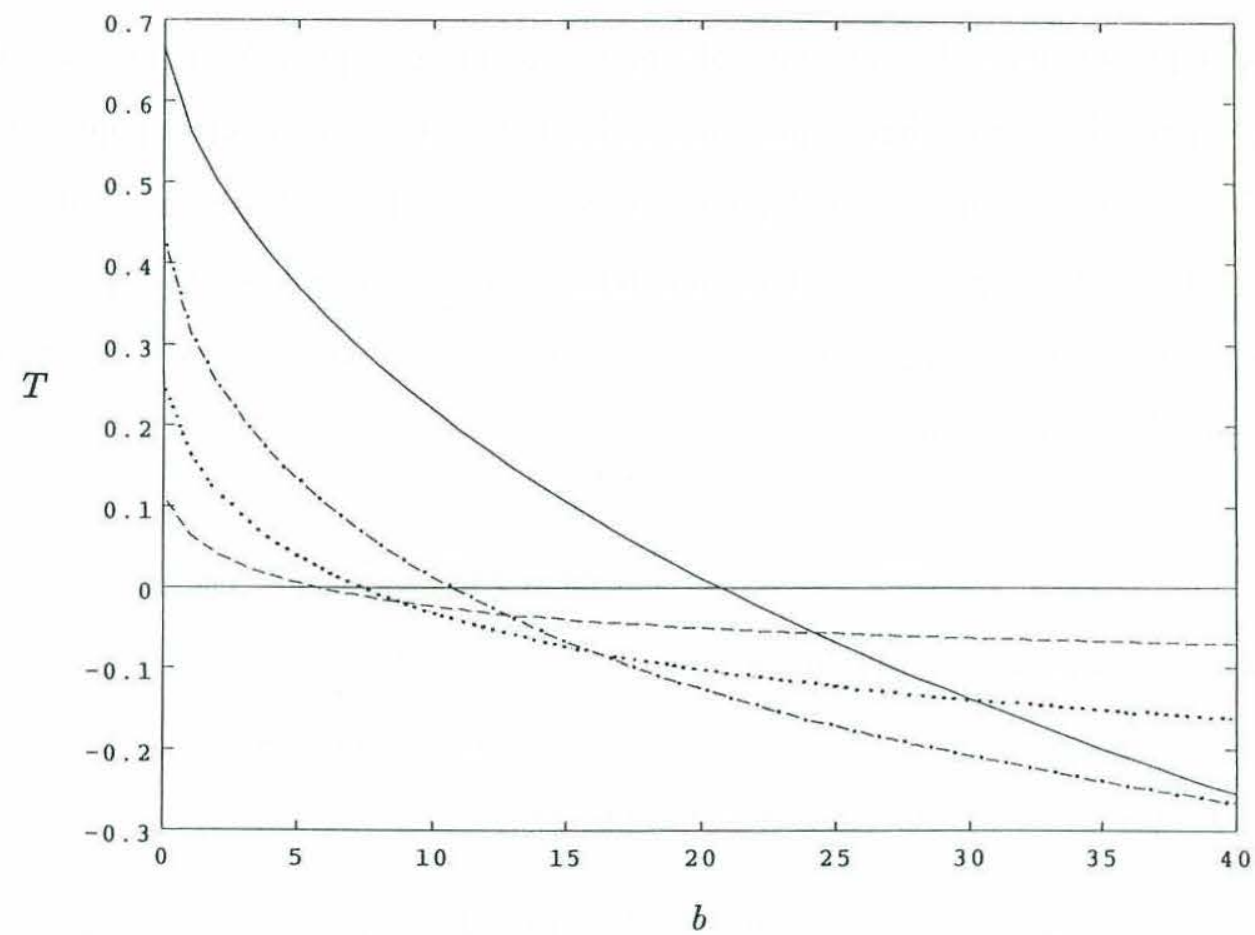

Figure 3.6: Blocking efficiency $T$ for westward flow for the shallow water solution for $h_{0}$ of 0.2 (dashed line), 0.4 (dotted line), 0.6 (dotted-dashed line) and 0.8 (solid line). Blocking efficiency for the quasi-geostrophic solution is 0 . 
Closed streamlines occur less easily for the shallow water model than for the quasi-geostrophic solution. The first stagnation point occurs on the boundary of the topography, as for the $\mathrm{f}-$ plane, and at $\theta=\pi / 2$. To find the critical height, $\psi_{r}$, at $\theta=\pi / 2, r=1$ is set to zero to get the transcendental equation

$$
\frac{1}{\left(1-h_{0}\right)}+b_{1} \kappa I_{0}^{\prime}(\kappa)+b_{2} \kappa I_{1}^{\prime}(\kappa)=0
$$

where $b_{1}$ is proportional to $1 / \epsilon$ and both $b_{1}$ and $b_{2}$ are complicated functions of $b$ and $h_{0}$. The critical height is then a function of both $b$ and $\epsilon$. However, a simple solution can be found for the critical Rossby number above which no closed streamlines exist. This is done by solving for $\epsilon$ in 3.19 and letting $h_{0}$ go to one as the upper limit on the height of the topography. Above this point, the critical height is greater than 1 , which is unphysical. Then

$$
\epsilon_{\max }=\frac{K_{1}\left(b^{1 / 2}\right)}{2\left(b^{1 / 2} K_{0}\left(b^{1 / 2}\right)+2 K_{1}\left(b^{1 / 2}\right)\right)},
$$

where we have substituted in the forms of $b_{1}$ and $b_{2}$. The $\mathrm{f}$-plane limit of $1 / 4$ can be found by letting $b$ go to zero. The critical Rossby number is a monotonically decreasing function of $b$ that approaches zero as $b \rightarrow \infty$.

For the quasi-geostrophic model, the critical height for closed streamlines to occur is given by

$$
h_{c r i t}=\frac{\epsilon}{I_{1}\left(b^{1 / 2}\right) K_{1}\left(b^{1 / 2}\right)} .
$$

A critical Rossby number can then be found for the quasi-geostrophic model by letting $h_{\text {crit }}=1$ in 3.20 (Figure 3.7). The quasi-geostrophic solution always gives a higher value than the shallow water model because the shallow water model allows the flow to be accelerated over the topography, so that it is harder for closed streamlines to form. For small Rossby numbers, it is unlikely that closed streamlines will develop for topographies with large horizontal extent in the barotropic model. 


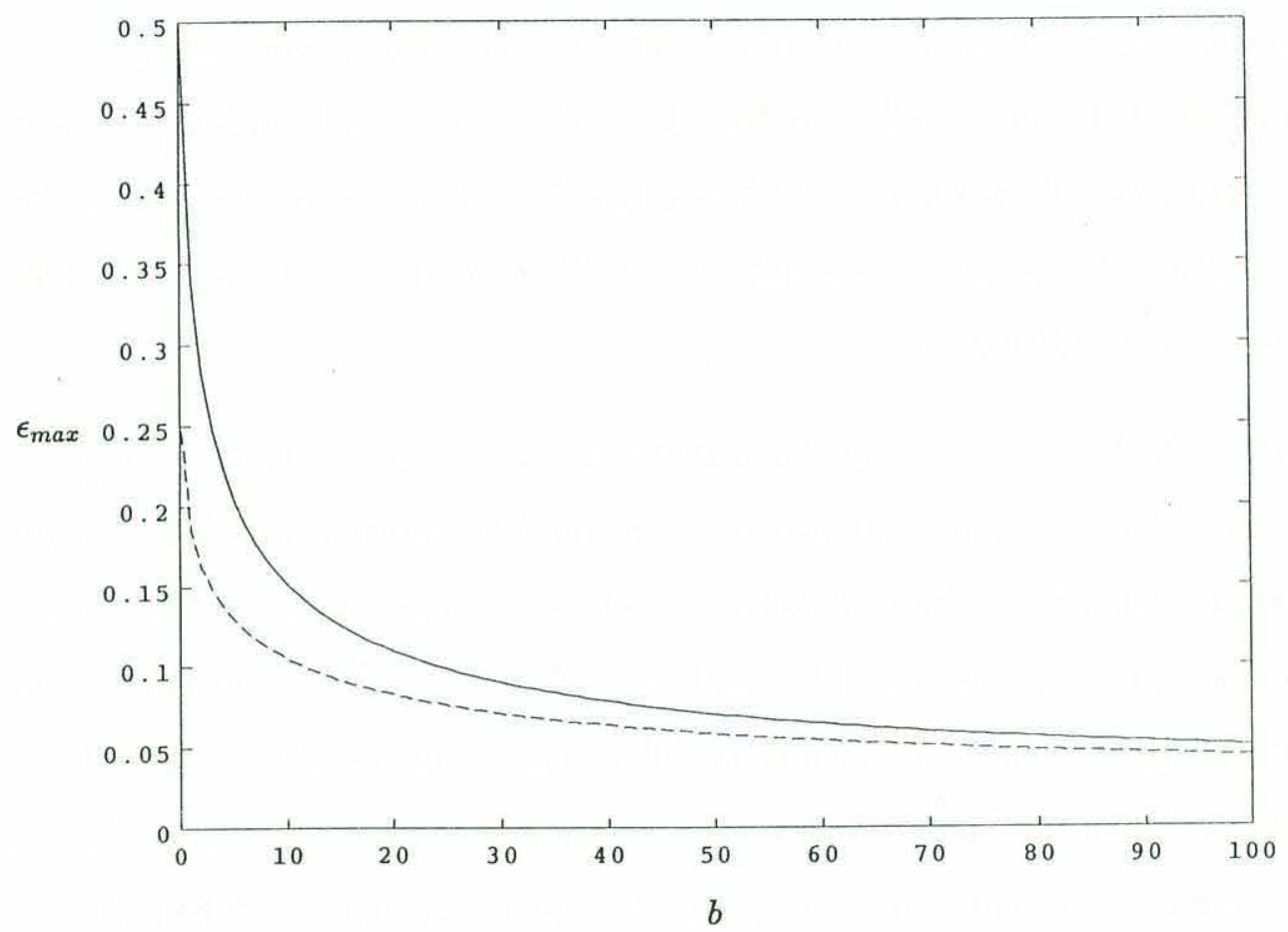

Figure 3.7: Maximum Rossby number for closed streamlines to occur for the quasigeostrophic (solid line) and the finite depth (dashed line) solutions for westward flow. 


\subsubsection{Solutions for eastward flow on the $\beta$-plane}

The behavior on the $\beta$ plane for eastward flow is very different from westward flow. For eastward flow, due to the anticyclonic vorticity over the topography, fluid parcels are forced to the north, this in turn increases the the potential vorticity due to the $\beta$ effect, and more anticyclonic relative vorticity is generated. Thus the $\beta$-effect and vortex squashing cannot balance each other. The excess relative vorticity induced causes the streamlines to move well away from their starting latitude and the Rossby restoring force generates downstream stationary Rossby waves, as can be seen in the wave-like solutions given below. Therefore, when the flow is toward the east, the additional boundary condition 3.6 must be applied.

In order to find the solution that satisfies the boundary condition we use the approach that Miles and Huppert (1968) took to find the solution in an analogous system of flow in a stratified fluid over a semi-circular obstacle. The resulting equation for the streamlines (in $z, x$ space) is identical to 3.5 . They constructed lee wave functions that satisfy the boundary condition individually, and then used these functions to find the solution. In fact, the solution that they found is exactly the solution for flow impinging on an island with zero circulation as described by McKee (1971). They required functions that were odd in $y$. For our problem both the even and the odd functions are needed.

The lee wave functions are constructed such that they each satisfy the boundary condition asymptotically and are given by

$$
\delta_{n}^{o}(r, \theta)=Y_{n}\left(b^{1 / 2} r\right) \sin n \theta+\phi_{n}^{o}(r, \theta)
$$

for the odd functions and

$$
\delta_{n}^{e}(r, \theta)=Y_{n}\left(b^{1 / 2} r\right) \cos n \theta+\phi_{n}^{e}(r, \theta)
$$


for the even functions where

$$
\phi_{n}^{o}(r, \theta)=\sum_{q=1}^{\infty} J_{q}\left(b^{1 / 2} r\right) \sin q \theta
$$

and

$$
\phi_{n}^{e}(r, \theta)=\sum_{q=1}^{\infty} J_{q}\left(b^{1 / 2} r\right) \cos q \theta
$$

The finite depth solution for $r>1$ can be written as a sum over the lee wave functions, and by changing the order of summation, we get that

$$
\psi=-r \sin \theta+\sum_{n=0}^{\infty}\left\{\left[\sum_{q=1}^{\infty} a_{q} Z_{n q}\left(b^{1 / 2} r\right)\right] \sin n \theta+\left[\sum_{q=0}^{\infty} c_{q} X_{n q}\left(b^{1 / 2} r\right)\right] \cos n \theta\right\}
$$

and

$$
\begin{aligned}
& Z_{n q}=\delta_{n q} Y_{n}\left(b^{1 / 2} r\right)+b_{q n} J_{n}\left(b^{1 / 2} r\right), \\
& X_{n q}=\delta_{n q} Y_{n}\left(b^{1 / 2} r\right)+d_{q n} J_{n}\left(b^{1 / 2} r\right)
\end{aligned}
$$

where

$$
b_{k l}= \begin{cases}\frac{4}{\pi} \frac{k}{l^{2}-k^{2}} & k \text { even, } l \text { odd } \\ \frac{4}{\pi} \frac{l}{l^{2}-k^{2}} & k \text { odd }, l \text { even } \\ 0 & k-l \text { even }\end{cases}
$$

and

$$
d_{k l}= \begin{cases}\frac{4}{\pi} \frac{l}{l^{2}-k^{2}} & k \text { even, } l \text { odd } \\ \frac{4}{\pi} \frac{k}{l^{2}-k^{2}} & k \text { odd, } l \text { even } \\ 0 & k-l \text { even } \\ \frac{4}{\pi} \frac{1}{l} & k=0, l \text { odd } \\ -\frac{2}{\pi} \frac{1}{k} & k \text { odd }, l=0 .\end{cases}
$$

Here $\delta_{i j}$ is the Kronecker delta. For $r<1$ the solution is

$$
\psi=-\frac{r \sin \theta}{1-h_{0}}-F_{0}+\sum_{n=0}^{\infty} J_{n}\left(k_{2} r\right)\left[b_{n} \sin n \theta+d_{n} \cos n \theta\right] .
$$

Applying the matching conditions gives an infinite set of coupled linear equations. They are comprised of two sets of equations for the $a_{q}$ 's and $b_{n}$ 's and two sets of 
equations for the $c_{q}$ 's and $d_{n}$ 's. In order to solve these, $n$ and $q$ are truncated at $N$, and then the set of equations is solved numerically. We let $N=10$, and it can be seen in the solutions constructed below that the upstream waves are small so that the upstream boundary condition has been satisfied.

The quasi-geostrophic solution is proportional to the first odd lee wave function and for $r>1$ can be written

$$
\left.\psi=r \sin \theta+\frac{2 h_{0}}{\epsilon b^{1 / 2}} J_{1}\left(b^{1 / 2}\right) S\left(b^{1 / 2}\right) r, \theta\right)-\frac{h_{0}}{\epsilon b^{1 / 2}}\left\{\frac{\pi}{2} S\left(b^{1 / 2} r, \theta\right) J_{1}\left(b^{1 / 2}\right) Y_{0}\left(b^{1 / 2} r\right)\right\}
$$

and for $r<1$

$$
\psi=r \sin \theta+\frac{2 h_{0}}{\epsilon b^{1 / 2}} J_{1}\left(b^{1 / 2}\right) S\left(b^{1 / 2} r, \theta\right)-\frac{h_{0}}{\epsilon b^{1 / 2}}\left\{\frac{\pi}{2} Y_{1}\left(b^{1 / 2}\right) J_{0}\left(b^{1 / 2} r\right)+\frac{1}{b^{1 / 2}}\right\}
$$

where

$$
S(\xi, \eta)=\sum_{n=1}^{\infty}\left[\frac{J_{2 n-1}(\xi) \cos ((2 n-1) \eta)}{2 n-1}\right]
$$

(McCartney, 1975). The circulation for the quasi-geostrophic solution is

$$
\Gamma=2 \pi \frac{h_{0}}{\epsilon} J_{1}\left(b^{1 / 2}\right) Y_{1}\left(b^{1 / 2}\right),
$$

reducing to the $\mathrm{f}$-plane result as $b$ approaches zero.

The drag from the quasi-geostrophic solution is given by

$$
D=2 \pi \epsilon\left(\frac{h_{0}}{\epsilon}\right)^{2} \frac{J_{1}^{2}\left(b^{1 / 2}\right)}{b^{1 / 2}} .
$$

The resulting drag has zeros in it, associated with resonant solutions as discussed by McCartney (1975). The envelope of the decay of the drag with increasing $b$ decreases algebraically as $1 / b$. As shown by Johnson (1977), the wave drag associated with eastward flow over topography depends critically on the form of the topography. Johnson (1977) shows that for smooth topography, $h=\left(1+r^{2}\right)^{-3 / 2}$ the wave drag falls off exponentially like $b^{1 / 2} e^{-b^{1 / 2}}$. Asymptotically, the drag for flow over Gaussian topography falls off like $b^{1 / 2} e^{-b}$. The abrupt topography discussed here is quite special 
because of the resonant solutions which result in the slow fall off of the drag with $b$, but the qualitative comparisons between the shallow water and quasi-geostrophic theory should still be valid for similar solutions of flow over smooth topography.

We apply the same restrictions on the parameters $(\epsilon b<1)$ as we did for the westward for cases. When $\epsilon$ is large, the solutions look quite similar to the westward flowing solutions (Figure 3.8). The streamlines move apart over the topography and the flow is partially blocked. There are very small amplitude waves downstream of the topography. For moderate $b$ and small $\epsilon$, the quasi-geostrophic solution and the shallow water solution look quite similar, with slightly larger amplitude Rossby waves and spreading of streamlines over the topography in the finite depth solution (Figure $3.9)$.

In the limit of large $b$, the shallow water and quasi-geostrophic solutions result in very different streamline patterns, as they did for westward flow (Figure 3.10). In this case, we cannot construct a boundary layer solution. Initially, parcels follow lines of constant background vorticity. This causes southward deflection of the streamlines. Because the deflection is larger in the finite depth solution due to the finite height of the topography, the stationary Rossby waves generated over the topography have larger amplitude as they compensate for the larger amount of relative vorticity generated. In the finite depth solution, both the even and the odd Rossby waves are forced, unlike the quasi-geostrophic solution. For the values of the parameters shown, the downstream waves have closed streamlines. These waves may not be stable, and would certainly be modified by frictional effects, but the solutions serve to show that large amplitude Rossby waves could be generated.

Remembering that the circulation is proportional to $1 / \epsilon$ we can directly compare the quasi-geostrophic solutions and the finite depth solutions (Figure 3.11). The magnitude of the circulation oscillates with a wavelength of approximately $b^{1 / 2}\left(1-h_{0}\right)$, the wave number of the variations over the topography. The circulation actually re- 


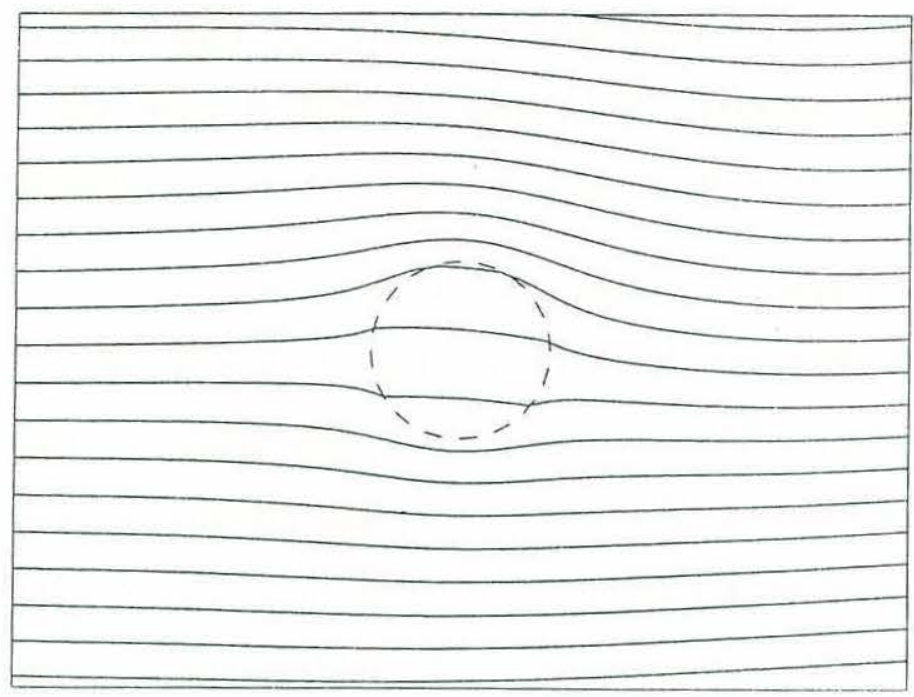

(a)

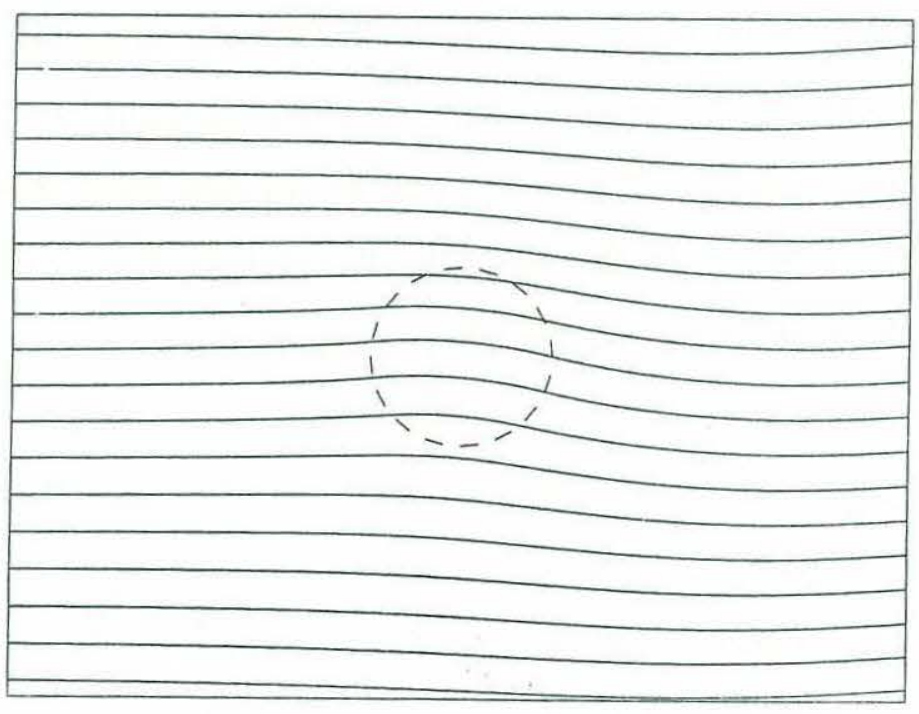

(b)

Figure 3.8: Stream function for eastward flow when $b=0.3, h_{0}=0.6$ and $\epsilon=2$. (a) Finite depth solution (b) quasi-geostrophic solution. These parameters are the same as shown in Figure 3.2 for westward flow. 


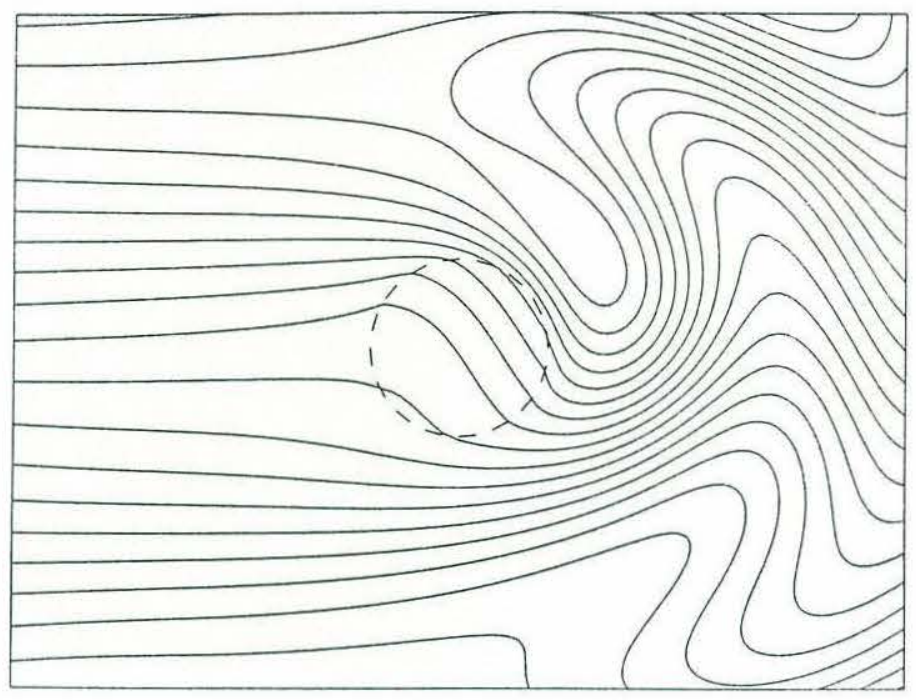

(a)

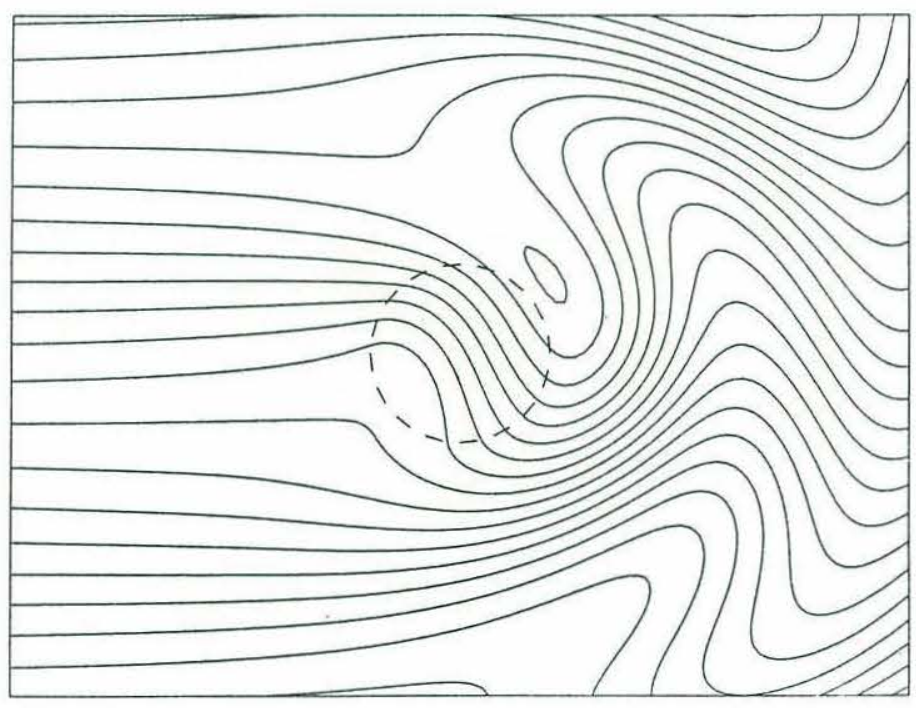

(b)

Figure 3.9: Stream function for eastward flow when $b=3, h_{0}=0.6$ and $\epsilon=0.2$. (a) Finite depth solution, (b) quasi-geostrophic solution. These parameters are the same as shown in Figure 3.1 for westward flow. 


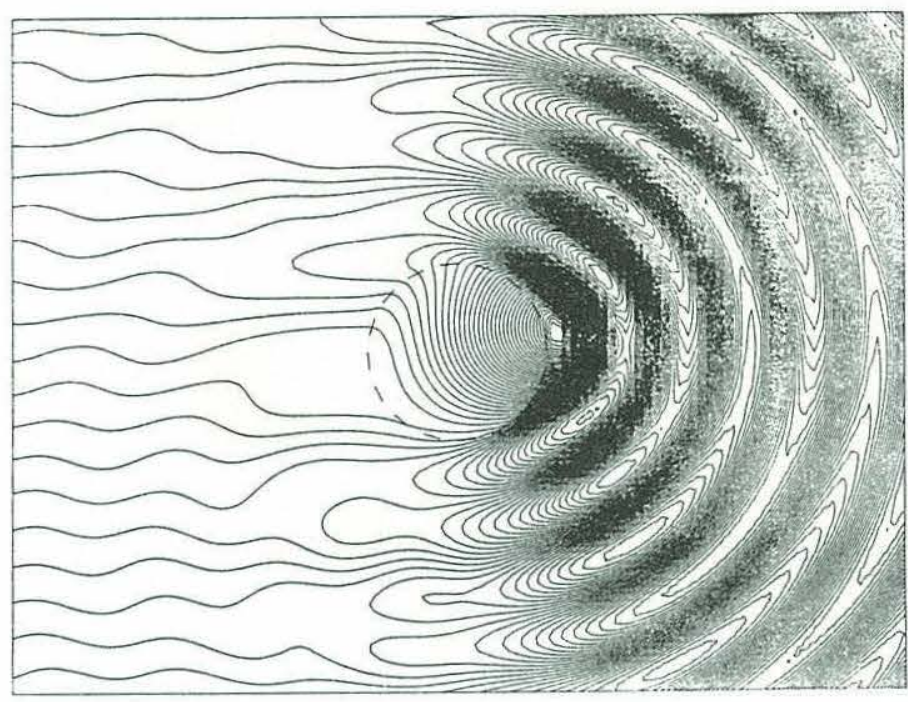

(a)

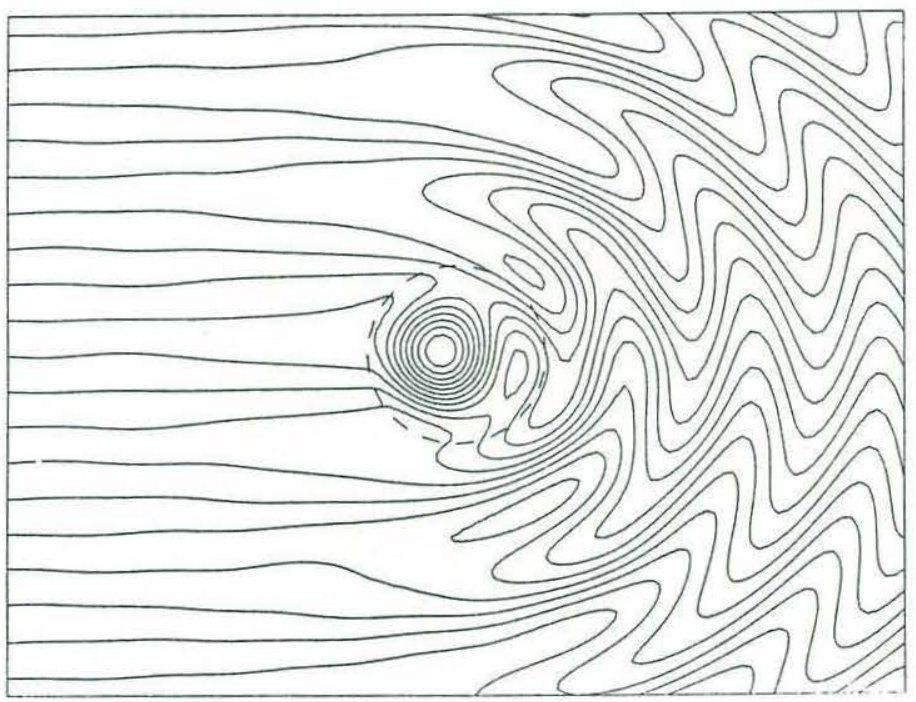

(b)

Figure 3.10: Stream function for eastward flow when $b=20, h_{0}=0.6$ and $\epsilon=0.5$. (a) Finite depth solution, (b) quasi-geostrophic solution. The stationary Rossby waves present upstream of the topography come from the error associated with the truncation of the solution at finite $N$ and are due to the fact that the downstream stationary Rossby waves have such large amplitude. 
verses sign due to local reversals of direction in the wave pattern as seen dramatically in Figure 3.10. It should be noted that it is very easy for closed streamlines to form in the wave field; this tendency calls into the question the validity of this form of steady solutions and also whether steady solutions can exist.

The blocking efficiency becomes negative for large $b$ as it did for the westward flow (Figure 3.12). This results once again from the tendency for the streamlines to follow lines of constant planetary vorticity when $b$ is large so that the flow is enhanced over the topography. However, this tendency does not increase indefinitely, because eventually the wave field has large enough amplitude and small enough wavelength to dramatically affect the flow locally over the topography (Figure 3.10). The blocking returns to zero when $b$ becomes large enough.

The lift increases with increasing $h_{0}$, and in all cases the lift for eastward flow on the $\beta$-plane is greater than the lift on the $\mathrm{f}-$ plane when $b=0$ (Figure 3.13). This effect was also seen for westward flow where the tendency for the odd portion of the wave function to dominate as $b$ increases causes larger values of the lift. The downstream stationary Rossby waves increase the lift, $L$, as a results of the rectification of the stream function associated with the quadratic terms in the pressure.

The drag is composed of two parts, one owing to the part of $P_{e}$ proportional to $1 / \epsilon$ and the other owing to the part proportional to $\epsilon$ (Figure 3.14). The first part of the drag has size comparable to the quasi-geostrophic drag, while the other part increases dramatically for increasing $h_{0}$. The second component does not contribute when $\epsilon \rightarrow 0$, but even for relatively small Rossby numbers (for instance $\epsilon=0.1$ ) it can be very large, due to the large amplitude Rossby waves generated downstream when both $b$ and $h_{0}$ are large. Thus drag calculated from the finite depth model has a very different character than that from the quasi-geostrophic model. The part of the pressure that contributes to the drag, $P_{e}$, has quadratic dependence on the stream 


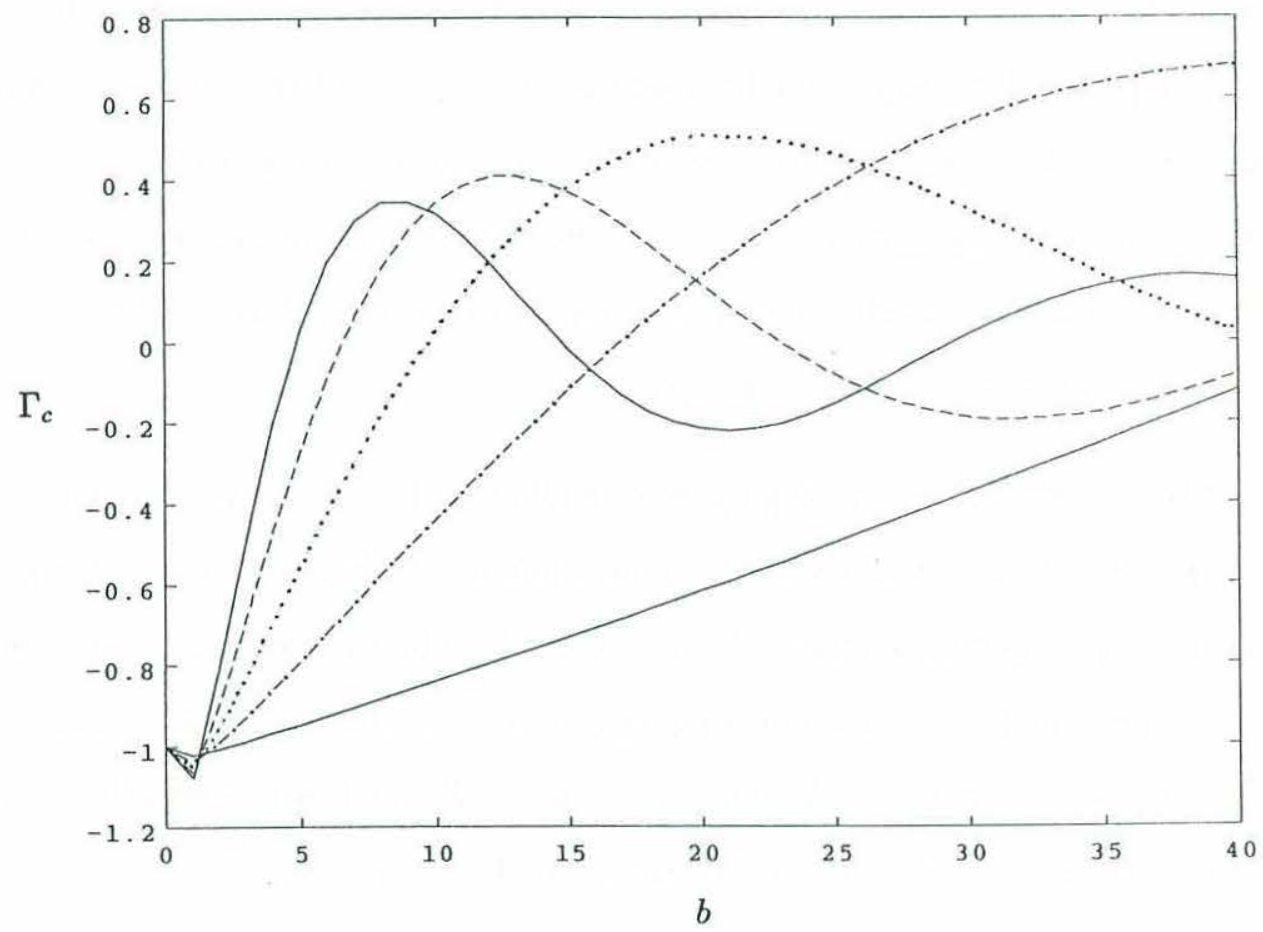

Figure 3.11: Circulation $\Gamma_{c}$ for eastward flow for the quasi-geostrophic solution (lower solid line), and for the shallow water solution for $h_{0}$ of 0.2 (dotted-dashed line), 0.4 (dotted line), 0.6 (dotted line), and 0.8 (lower solid line). It is shown in units of $h_{0} \pi / \epsilon$. 


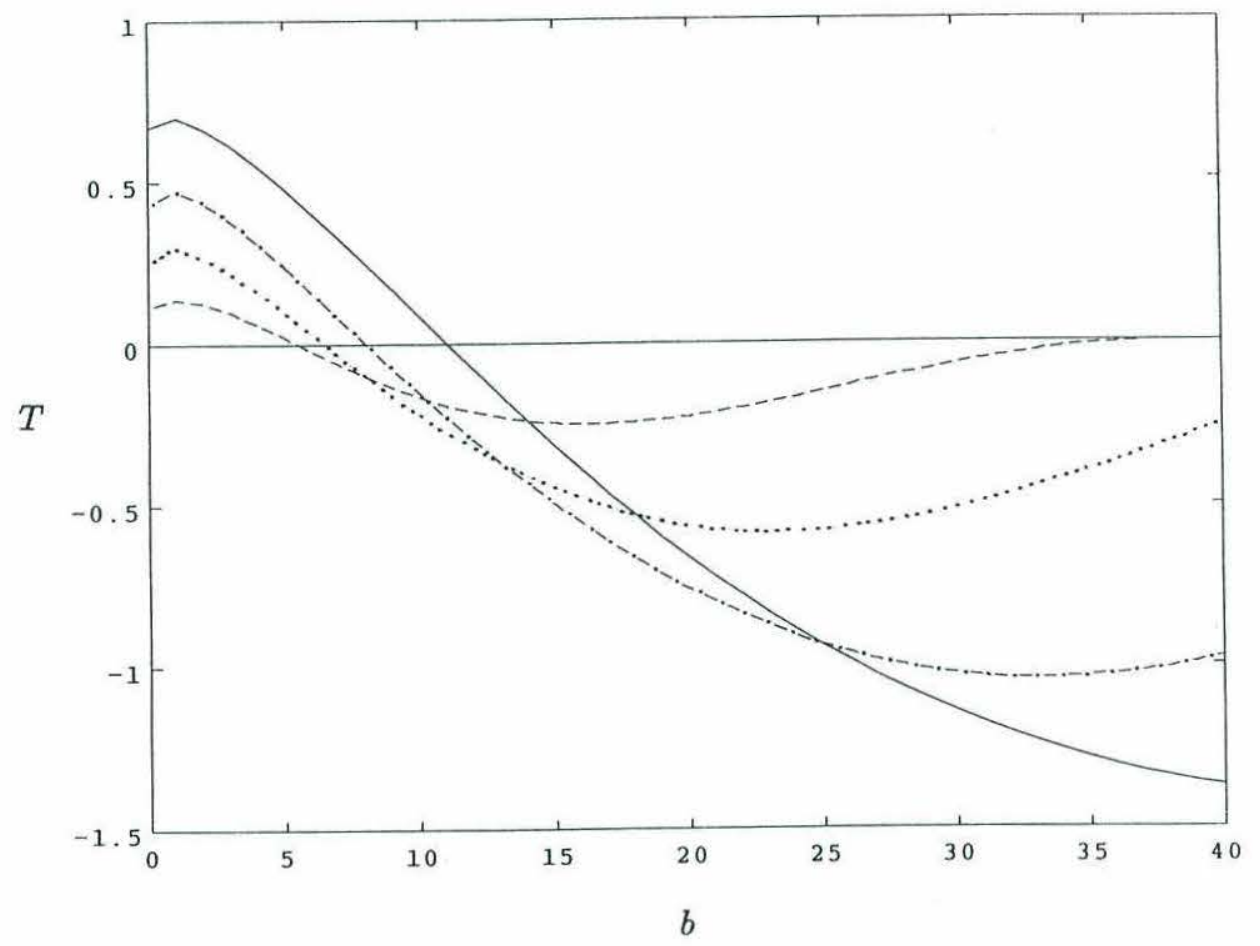

Figure 3.12: Blocking efficiency $T$ for eastward flow for the shallow water solution for $h_{0}$ of 0.2 (dashed line), 0.4 (dotted line), 0.6 (dotted-dashed line), and 0.8 (solid line). Blocking efficiency for the quasi-geostrophic solution is 0 . 


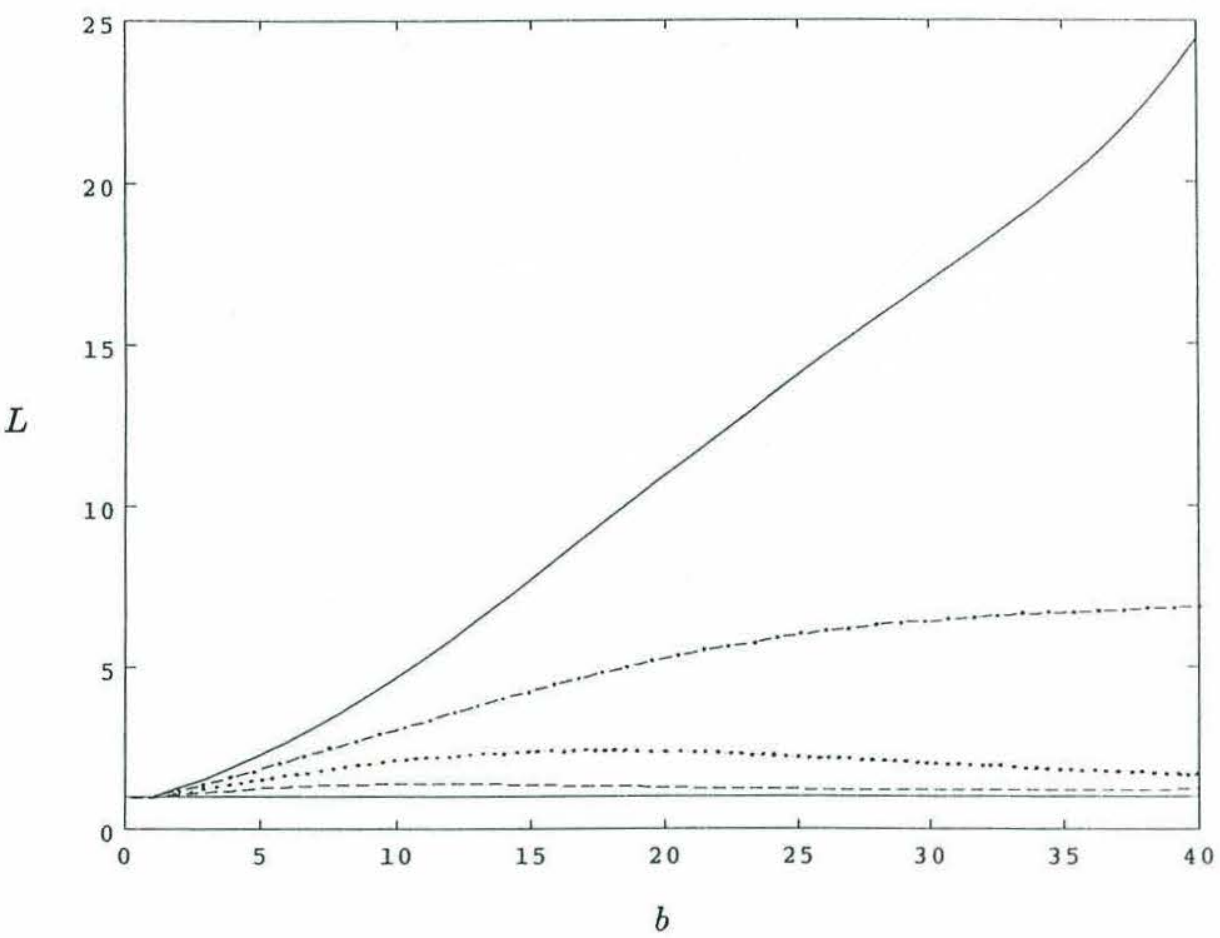

Figure 3.13: Lift $L$ for eastward flow for the quasi-geostrophic solution (lower solid line), and for the shallow water solution for $h_{0}$ of 0.2 (dotted-dashed line), 0.4 (dotted line), 0.6 (dotted line), and 0.8 (solid line). It is shown in units of $\pi$ so that the quasigeostrophic lift has magnitude 1. 
function, and the downstream wave field is rectified in this calculation. The second component (depending on $\epsilon$ ) is large because it depends on $\psi_{o}$.

\subsubsection{Summary: barotropic flow over finite topography}

For flow over finite topography in a barotropic fluid, quasi-geostrophic theory tends to underestimate the strength of the flow over the topography resulting in an underestimate of the circulation, lift, and drag of the flow. In addition, it causes the critical Rossby number above which closed streamlines cannot exist above the topography to be larger than found in the finite depth model.

The most dramatic differences between the two solutions can be seen at large values of $b$. For either westward or eastward flow, the blocking becomes negative, and the amount of fluid going over the topography is enhanced relative to the background flow. In this parameter regime, the fluid parcels approximately follow lines of constant background vorticity so that the streamlines are deflected to the south over the topography in both the quasi-geostrophic and finite depth models. However, the southward deflection is larger in the finite depth model.

The other difference is that due to the quadratic terms in the pressure. The forces on the topography are underestimated by quasi-geostrophic theory. In particular for eastward flow, the Rossby wave drag can potentially be much larger than the quasi-geostrophic estimate, particularly when both $b$ and $h_{0}$ are large.

A closing word on parameter values helps to put the above results in perspective. In the ocean, topographic features are often quite tall, so the dependence on $h_{0}$ is important. However, tall seamounts generally have rather small horizontal extent (for example, 50 to $100 \mathrm{~km}$ ). This results in a value of $b$ which is order one. The solutions with large $b$ discussed may be relevant for flow over the largest scales of 


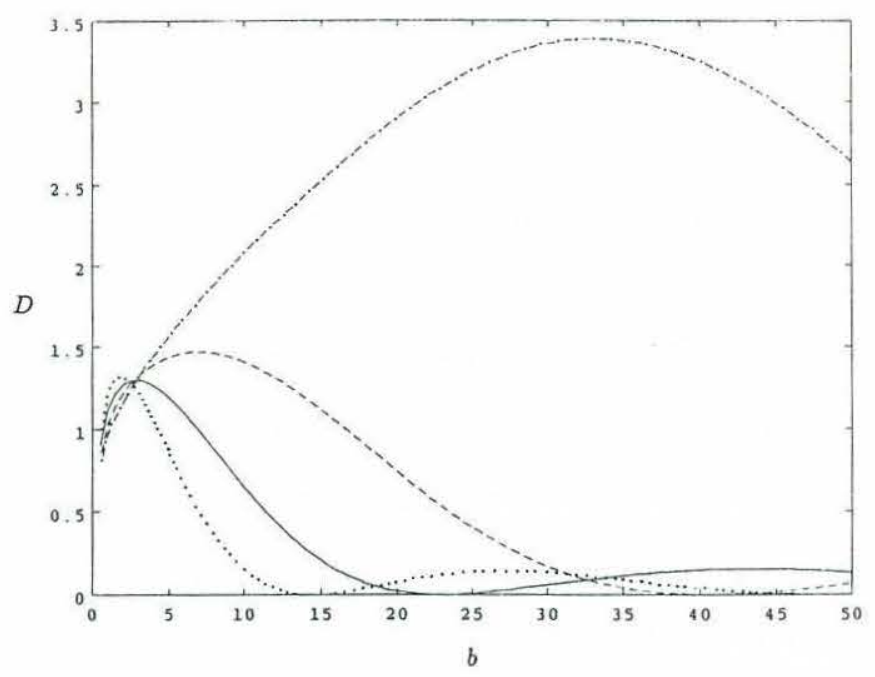

(a)

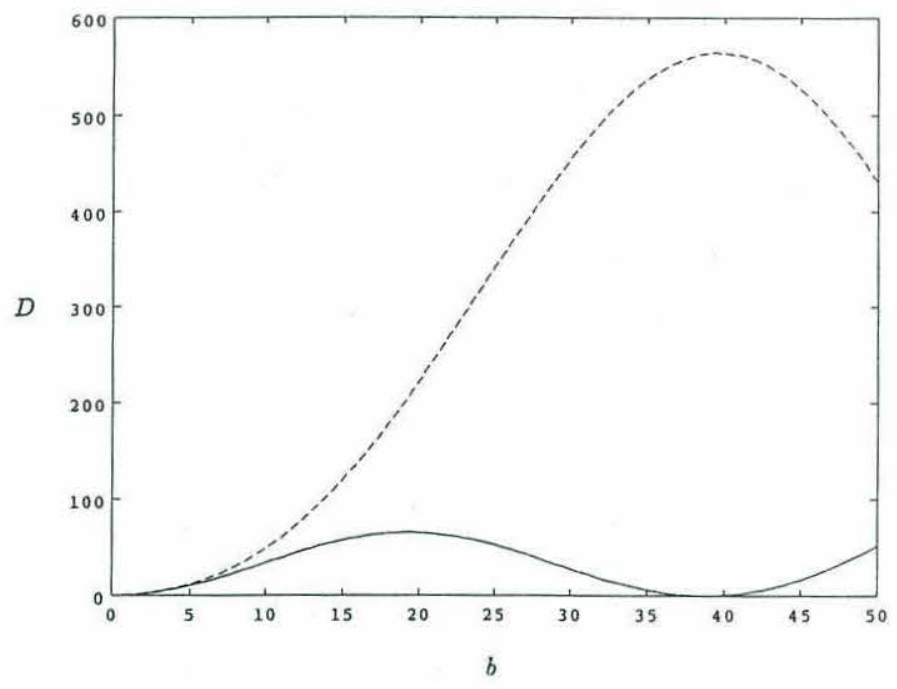

(b)

Figure 3.14: Drag $D$ for eastward flow. (a) The portion proportional to $1 / \epsilon$ for the quasi-geostrophic solution (dotted line), $h_{0}$ of 0.2 (solid line), 0.4 (dashed line), 0.6 (dotted-dashed line), (b) the portion proportional to $\epsilon$ for $h_{0}$ of 0.2 (solid line), 0.4 dashed line. The quasi-geostrophic contribution is zero. For both plots, the drag is in units of $h_{0}$. 
topography which interacts with the slow abyssal flow (e.g. Straub and Rhines, 1990, and Rhines, 1989), and it is in this regime for which we find the most differences between the quasi-geostrophic and finite depth solutions. In a quasi-geostrophic model of the circumpolar current, Treguier and McWilliams (1990) show that the largest scales of topography contribute most to the drag on the current. Our study suggests that this effect may be even more dramatic when non quasi-geostrophic effects are allowed. 


\subsection{Flow over finite topography in a two-layer model}

In Section 3.3 we explored what happens when the topographic feature is of finite height in a barotropic fluid. The solution is comparable to the quasi-geostrophic solution as long as both the topography and the flow are small enough. As soon as the topography becomes large, the quasi-geostrophic solution is inaccurate due both to the horizontal divergence of the flow allowed by the shallow water solution, and to the finite Rossby number effects on the pressure. An indication of this inaccuracy is given by the comparison of integral properties of the flow between the finite depth solution and the quasi-geostrophic solution. The next step in understanding flow over finite topography in the ocean is to include stratification.

Often, seamounts fill a large fraction of the water column, and one would like to know the effect of this on oceanic flow in a more realistic model. Unfortunately it is not possible to do a simple model with an analytic solution similar to the one described in Section 3.3 using the shallow water equations for either a layered fluid or a continuously stratified fluid. In a two layer fluid, the interface height non linearly couples the flow, and the flow is no longer simply given by the solution of linear partial differential equations. This complication also manifests itself in continuously stratified models. The problem of flow impinging on topography of finite height in a continuously stratified fluid has been solved numerically using the primitive equations; however the steady solution has only been found on the f-plane and the solution is found by allowing a time-dependent model to reach steady state (Chapman, personal communication). Needless to say this process is intensive in computer resources. We would like to develop a dynamically simple theory which will help in the study of primitive equation models by giving simple predictions as to what happens when a stratified fluid interacts with finite topography. 
The quasi-geostrophic steady solutions that have been studied to the present have concentrated on flow in which the lower boundary is an isopycnal surface, so that isopycnals do not intersect the topography and thus have limited applicability for oceanic flows over seamounts which reach nearly to the surface (i.e. Buzzi and Speranza, 1979, Schar and Davies, 1988). Here, we consider a fluid consisting of two layers in which the topography goes all of the way through the lower layer and extends only slightly into the upper layer so that the quasi-geostrophic approximation is valid. The problem can be solved analytically using the same mathematics as described in Section 3.3. In this way we are able to describe flow over finite topography in a stratified fluid analytically.

In order to make use of this model, we would like to make a connection between a model with this geometry and one with more realistic geometry such as smooth topography and continuous stratification so that comparisons and predictions can be done with it. Under the quasi-geostrophic approximation, the flow must remain nearly horizontal. When the flow reaches an obstacle, depending on the slope of the obstacle and the stratification, the flow can either go over the obstacle or around it. Buzzi and Speranza (1979) showed through a dimensional argument that when $h_{0} / \epsilon \lesssim 1 / S$, where $S$ is the Burger number, the flow can go up and over the obstacle, and the quasi-geostrophic solution can be found by applying the bottom boundary condition on the vertical velocity. When $h_{0} / \epsilon \gtrsim 1 / S$ the flow must go around instead of over the obstacle in order to keep the flow horizontal, and the boundary condition at the topography is applied on the horizontal velocity. Thus, quasi-geostrophic theory can be applied in these two situations. When the Rossby number is larger such that the flow goes up the topography, quasi-geostrophic theory will not be adequate for describing the flow. There is a distinction here between the flow going around the topography to remain quasi-horizontal, and closed streamlines appearing over the topography in a stratified fluid which result in the trapping of fluid. In a stratified model, both things can occur, and they happen at different values of the parameters 
as Schar and Davies (1988) showed. Quasi-geostrophic theory allows a wall with large enough slope to be approximated by a vertical face, so for a slowly moving fluid (small Rossby number), our geometry is reasonable.

Buzzi and Speranza (1979) solved for the flow around a vertical disk that obstructs the flow. This obstacle has a finite vertical cross section, but a small horizontal cross section. In their continuously stratified model, they show that at a vertical wall the stream function depends only on the vertical coordinate, and they choose, somewhat arbitrarily, that the stratification be constant on the wall. This is equivalent to requiring that the isopycnals are flat at the wall. In our model, we have finite cross sections both in the vertical and horizontal, extending Buzzi and Speranza's (1979) work, and we solve for the flow in the two-layer model. We must choose the circulation around the obstacle in the lower layer, and this choice is arbitrary. We choose for this chapter to have no circulation in the lower layer.

Two-layer models can be calibrated according to the real oceanographic stratification (Flierl, 1978), and this calibration depends on what phenomenon is of interest. It is not clear exactly how the model that we study here would be calibrated, but we guess that the interface location would approximately correspond to the location of the node in the first baroclinic mode. A more detailed calibration has not been done. The model is probably more accurate for a situation in which the stratification is localized in the vertical and above the region of strong stratification the fluid is relatively unstratified. A measure of this would be the scale at which motions are bottom trapped $\left(H_{s}=f_{0} L / N\right)$ verses the depth of the upper layer. If $H_{s}$ is much greater than the depth of the upper layer, we would expect that our model would be relevant.

For this two-layer model, we consider a variety of strengths of the stratification parameter. The relative layer depth is also an adjustable parameter. We choose to consider two cases: equal layer depths, so that a comparison can be made to a 
simpler two-layer model, and an upper layer depth equal to 0.2 times the lower layer depth. This choice is traditionally chosen for the interface height to correspond to the location of the thermocline in the ocean. There are several places in the ocean where seamounts penetrate nearly to the surface, where one would like to apply this model, such as flow of the Kuroshio over the Emperor Seamounts, flow of the Gulf Stream over the New England Seamounts, and flow over isolated guyots that reach nearly to the surface.

The model is physically consistent as long as two conditions hold. When closed streamlines occur, the inviscid theory no longer applies on time scales comparable to the viscous spin up time $\tau$. As shown in Chapter 2 for a one-layer fluid, a new solution can be found such that under the influence of Ekman friction, the fluid within closed streamlines becomes stagnant. This breakdown criterion tells when we expect fluid to be trapped over the topography. If the interface goes above the topography, the solution no longer applies. This breakdown criterion gives an indication of how a more general stratified model would behave. This is approximately the same as the breakdown criterion of the continuously stratified quasi-geostrophic models reviewed earlier in which the breakdown criterion is associated with the application of the bottom boundary condition (Schar and Davies 1988, Buzzi and Speranza 1979, Merkine and Kalnay-Rivas 1976). In continuously stratified models, the requirement is that the bottom boundary remains isopycnal.

In the sections that follow, the model formulations are presented. First, we consider a barotropic incoming flow on the f-plane, then flow on the f-plane with shear, and finally examples are given of both westward and eastward flow on the $\beta$-plane. 


\subsubsection{Model formulation}

The formulation of the problem follows closely that of McCartney (1975) for his solution of two-layer flow over small topography on the $\beta$-plane. We use the scaling that he developed in his paper. The only difference is that we let the upper layer be indexed as layer 1 and the lower layer as layer 2, opposite to his notation but consistent with the notation in the rest of this thesis and other more recent work done with the two layer model. The basic geometry of this model as well as McCartney's (1975) model as shown in Figure 1.1.

As McCartney shows, the parameters in this inviscid theory are

$$
\begin{gathered}
\epsilon=\frac{U_{0}}{f_{0} L}, \\
b=\frac{\beta L^{2}}{U_{0}}, \\
h_{0}=\frac{h_{0}^{\prime}-d^{\prime}}{H}, \\
S=\frac{g H \Delta \rho}{f_{0}^{2} \rho_{0} L^{2}}, \\
d=\frac{d^{\prime}}{H}
\end{gathered}
$$

and

$$
U_{0}^{2}=(1-d) \mathcal{U}_{1}^{2}+d \mathcal{U}_{d}^{2}
$$

where $\mathcal{U}_{1}$ and $\mathcal{U}_{2}$ are the velocities in the upper and lower layer, respectively.

As in Section $3.3 \epsilon$ is the Rossby number, but here using the depth averaged root mean square velocity, $b$ is a measure of the importance of $\beta$, the ratio of the long Rossby wave speed to the background flow speed, $h_{0}$ is a measure of the topographic height variation in the upper layer, $S$ is a measure of the importance of the stratification and is equivalent to the Burger number for this two-layer model, and $d$ is the scaled depth of the lower layer. When $S$ is large, the stratification is strong and the 
two layers are relatively uncoupled; when $S$ is small, the stratification is weak and the two layers are strongly coupled. Under the quasi-geostrophic approximation, $S$ is assumed to be order one and the Rossby number is small. In addition, $h_{0}$ is of order Rossby number. This last requirement allows the dynamics to be internally consistent so that the layer depth changes only an order Rossby number amount, the flow remains quasi-horizontal, and the two layers are coupled linearly.

Under the quasi-geostrophic approximation, the two-layer model is governed by the two-layer potential vorticity equations. The horizontal velocities are scaled by $U_{0}$ and the horizontal length is scaled by $L$, the length scale of the topography. The two-layer equations become

$$
\partial_{t} q_{n}+J\left(\psi_{n}, q_{n}\right)=0
$$

where $q_{n}$ indicates the quasi-geostrophic potential vorticity in the $\mathrm{n}$-th layer, where away from the topography

$$
q_{1}=\nabla^{2} \psi_{1}+b y+\frac{\psi_{2}-\psi_{1}}{S(1-d)}
$$

and

$$
q_{2}=\nabla^{2} \psi_{2}+b y+\frac{\psi_{1}-\psi_{2}}{d S} .
$$

The interface height difference away from its mean depth is given by

$$
\eta=\epsilon \frac{\psi_{2}-\psi_{1}}{S}
$$

and is an order Rossby number quantity.

In steady state, 3.22 allows a solution such that $q_{n}$ is a function of $\psi_{n}$ for $n=1,2$. The functional relationship between the potential vorticity and the stream function in each layer is determined by the upstream conditions. Upstream, $\psi_{n}=$ $-U_{n} y$. Thus using 3.23

$$
q_{1}=-\frac{\left(b+\frac{U_{2}-U_{1}}{S(1-d)}\right)}{U_{1}} \psi_{1},
$$


and likewise using 3.24

$$
q_{2}=-\frac{\left(b+\frac{U_{1}-U_{2}}{S d}\right)}{U_{2}} \psi_{2} .
$$

With uniform flow upstream of the topography in each layer we can let $\psi_{n}=-U_{n} y+$ $\phi_{n}$. Away from the topography we have from 3.26 and 3.27

$$
\nabla^{2} \phi_{2}-\phi_{2}\left[-\frac{b}{U_{2}}+\frac{U_{1}}{U_{2} d S}\right]=-\frac{\phi_{1}}{d S}
$$

and

$$
\nabla^{2} \phi_{1}-\phi_{1}\left[-\frac{b}{U_{1}}+\frac{U_{2}}{U_{1}(1-d) S}\right]=-\frac{\phi_{2}}{(1-d) S} .
$$

This is the same set of equations that McCartney (1975) uses to describe steady flow over topography of small (order Rossby number) height.

However, with this geometry, over the topography the fluid obeys a one layer equation. In general, the governing equation is given by

$$
\partial_{t} q_{1}+J\left(\psi_{1}, q_{1}\right)=0
$$

where

$$
q_{1}=\nabla^{2} \psi_{1}+\frac{h-d}{\epsilon(1-d)} .
$$

The same functional relationship as in 3.26 should hold between $q_{1}$ and $\psi_{1}$. Thus for $r<1$ we let

$$
\psi_{1}=-\frac{y U_{1} b}{b+\frac{U_{1}-U_{2}}{S(1-d)}}+\phi_{2}
$$

so that

$$
\nabla^{2} \phi+\phi_{2} k_{3}^{2}=-\frac{h_{0}-d}{\epsilon(1-d)}
$$

where

$$
k_{3}^{2}=\frac{b+\frac{U_{1}-U_{2}}{S(1-d)}}{U_{1}} .
$$

The sign of $k_{3}^{2}$ depends on the direction of the flow. For the cases that we consider $\left(U_{1}>U_{2}\right)$ for eastward flow and f-plane flow, $k_{3}^{2}>0$ indicating a trapped wave response. However, for westward flow, $k_{3}^{2}$ can have either sign. 
McCartney (1975) considered for simplicity the flow over a right circular cylinder whose depth is small compared to the total depth, and is only in the lower layer. In order to solve the problem, he found an equation for $\psi_{1}$ and required that $\psi_{1}$ and its first three derivatives in $r$ be continuous at $r=1$, equivalent to the velocity being continuous at $r=1$ in both layers. Here, the requirements are that the velocity must be continuous in both directions in the upper layer, and the velocity normal to the cylinder must vanish in the lower layer $\left(\psi_{2}=\right.$ constant at $\left.r=1\right)$. However, there is still an undetermined constant which is chosen by specifying the circulation around the topography in the lower layer. We choose the circulation to be zero in the lower layer at $r=1$. This last condition makes sense because this solution corresponds to the steady state solution of an initial value problem whereby the velocity is brought to the final value from rest. If initially there is no circulation, and if the fluid remains inviscid throughout its evolution, then no circulation develops. This is the same choice that has been made for the solution to the problem of flow impinging on an island (White, 1971; and McKee, 1971). The lower layer is coupled to the upper layer, and so it is not simply the solution that White (1971) and McKee (1971) found. The circulation around the cylinder is also related to the lift and drag on the cylinder in the lower layer. When the circulation is zero, there is no lift or drag in the lower layer.

The solution can be separated into an island component (odd in $y$ ) and a topographic component (even in $y$ ). For the problem that McCartney (1975) studied, the odd portion of the solution is composed of the background flow only. This separation is similar to what we find for the solution of flow of a barotropic fluid over finite topography. For the two-layer model, the odd component is forced by the boundary conditions in the lower layer, while the even component is forced by the topographic contribution to the potential vorticity anomaly. We are thus capturing in this simple geometry one of the elements that quasi-geostrophic theory of flow in a stratified fluid in which the bottom always remains an isopycnal lacks: that the flow over an 
axisymmetric obstacle of finite height is composed of two components with different symmetry in $y$, and splitting of the flow around the topography occurs. Buzzi and Speranza's (1979) solution of flow impinging on a vertical disk lacks the symmetric component. We are capturing both components in the solutions that we show below.

For $r>1$ the fluid responds much in the way described by McCartney (1975). The governing equation for the flow in the upper layer is then for $r>1$

$$
\left(\nabla^{2}+k_{1}^{2}\right)\left(\nabla^{2}+k_{2}^{2}\right) \psi_{1}=0
$$

where $k_{1}$ and $k_{2}$ are given by

$$
k_{1,2}^{2}=-\frac{1}{2}\left(N_{1}+N_{2}\right) \pm \frac{1}{2}\left(\left(N_{1}-N_{2}\right)^{2}+4 N_{3}\right)^{1 / 2}
$$

where

$$
\begin{gathered}
N_{1}=-\frac{b}{U_{2}}+\frac{U_{1}}{U_{2} d S}, \\
N_{2}=-\frac{b}{U_{1}}+\frac{U_{2}}{U_{1}(1-d) S}
\end{gathered}
$$

and

$$
N_{3}=\frac{1}{d(1-d) S^{2}}=\Gamma^{2}
$$

When $k_{1}^{2}$ or $k_{2}^{2}$ are negative we define

$$
\kappa_{1}^{2}=-k_{1}^{2}
$$

and

$$
\kappa_{2}^{2}=-k_{2}^{2}
$$

The fundamental length scales of the problem are set by $k_{1,2}$. When the background velocity is barotropic and $b=0$ then the wavenumbers are 0 and $\Gamma$, the ratio of $L$ to the baroclinic Rossby radius of deformation. McCartney (1975) showed that depending on the choice of the parameters $k_{1}^{2}$ and $k_{2}^{2}$ can either be zero, positive, or negative. When $k_{n}^{2}$ is negative, the stream function falls off exponentially as 
evanescent Rossby waves or as a trapped baroclinic response; when $k_{n}^{2}$ is positive, a stationary Rossby wave solution is allowed. Fandry et al. (1983) considered solutions when the shear is reversed. In this study, we restrict the solutions to the case of larger velocity in the upper layer than the lower layer. As in Section 3.3.5, we construct the solution out of lee-wave functions, applying the matching conditions as outlined above. It should be noted that as the condition for the background flow to be stable we must have

$$
U_{1}-U_{2}<b d S \text {. }
$$

For some of the cases that we are considering, baroclinic instability of the background flow is a possibility. In these circumstances the steady solution may not be realizable.

To help understand the solutions we also study critical heights of the problem. We first note however, that the wave-drag will be similar to the quasi-geostrophic model, but the lift calculations will be fundamentally different, since there are antisymmetric components of the flow besides the background flow. The lift and drag are only non-zero in the upper layer due to the requirement that there is no circulation in the lower layer.

\subsubsection{Barotropic flow on the $f$-plane}

The solution when the incoming flow is barotropic, and the topography is relatively small in horizontal extent so that $\beta$ can be ignored is discussed in this section.

In this case, $k_{1}$ is zero (the barotropic mode) and $\kappa_{2}=\Gamma$ where for $r>1$ we have

$$
\psi_{1}=a_{1} \ln r+a_{2} K_{0}(\Gamma r)+a_{3} \sin \theta / r+a_{4} K_{1}(\Gamma r) \sin \theta-r \sin \theta
$$

and for $r<1$

$$
\psi_{1}=b_{1}-\frac{h_{0}-d}{4 \epsilon(1-d)} r^{2}+b_{2} r \sin \theta
$$


while

$$
\psi_{2}=a_{1} \ln r-\delta a_{2} K_{0}(\Gamma r)+a_{3} \sin \theta / r-\delta a_{4} K_{1}(\Gamma r) \sin \theta-r \sin \theta
$$

where $\delta=H_{1} / H_{2}=(1-d) / d$. Applying the matching conditions we find that

$$
\begin{gathered}
a_{1}=-\frac{q_{a} \delta}{2(1+\delta)}, \\
a_{2}=\frac{q_{a} \delta K_{0}(\Gamma)}{2(1+\delta) K_{1}(\Gamma)+K_{0}(\Gamma)}, \\
a_{3}=\frac{2 K_{1}(\Gamma)+K_{0}(\Gamma)}{2(1+\delta) K_{1}(\Gamma)+K_{0}(\Gamma)}, \\
b_{1}=q_{a}\left(\frac{K_{0}(\Gamma)}{2 \Gamma K_{1}(\Gamma)}+\frac{1}{4}\right)
\end{gathered}
$$

and

$$
b_{2}=\frac{2(1+\delta) K_{1}(\Gamma)}{2(1+\delta) K_{1}(\Gamma)+K_{0}(\Gamma)} .
$$

We have defined

$$
q_{a}=\frac{h_{0}}{\epsilon(1-d)},
$$

which is the magnitude of the potential vorticity anomaly in the upper layer. Notice that $a_{3}$ and $b_{2}$ are independent of $q_{a}$.

When the stratification is moderate, the solution contains both bump-like components (those independent of $\theta$ ) and island-like components (those proportional to $\sin \theta)$ (Figure 3.15 ). The additional angle dependent components result in an interface which is asymmetric and is higher in the north than in the south, while the fluid is split by the topography in the lower layer. The asymmetric response of the interface is consistent with what happens in the primitive equation models (Chapman, personal communication); however, in this model, the interface near the topography in the south is depressed, whereas in quasi-geostrophic theory in which the isopycnals do not intersect the topography, the isopycnals rise over the topography. The interface is depressed because it is responding to the anticyclonic perturbation in the upper 
layer and no circulation in the lower layer. If we let $q_{a}=0$ then the interface would be tilted symmetrically north-south, since all of the radially symmetric terms in the solution would vanish. The solutions can be compared to McCartney's (1975) solution, which contains the bump like components plus the background flow (Figure 3.16). In this case, the interface is raised uniformly over the topography as a radially symmetric perturbation.

To understand the solutions further, we can calculate the vertical velocity at the interface which is given in the two-layer model by

$$
w_{1}=\frac{d \eta}{d t}=\frac{\epsilon}{S} J\left(\psi_{1}, \psi_{2}-\psi_{1}\right)
$$

The vertical velocity is shown in Figure 3.17 for our model and for the small topography model. The vertical velocity is positive upstream of the obstacle, and negative downstream as the fluid goes up and over the obstacle. There is an asymmetric response (north-south asymmetry) in the finite depth model.

When $\delta=0.2$ the solution looks different. Since the lower layer is deeper, the flow more nearly looks like the solution of irrotational flow about a cylinder. There are more closed streamlines in the upper layer (Figure 3.18).

From the solution given by $3.32,3.33$, and 3.34 , two critical heights of the problem can be found. First we find the topographic height such that the maximum of the interface height $\eta$ from 3.25 and the topographic height are equal. This is given by

$$
\frac{h_{\eta}}{\epsilon}=\frac{4 \Gamma^{2} K_{1}(\Gamma)^{2} \delta \Gamma}{\left(2 \sqrt{\delta} K_{1}(\Gamma)+K_{0}(\Gamma)\right)\left(2 K_{1}(\Gamma)(1+\delta)+K_{0}(\Gamma) \Gamma\right)} .
$$

The maximum interface height always occurs at $\theta=\pi / 2$ (in the north for eastward flow) and $r=1$ for eastward flow on the f-plane. For a physically consistent solution we must require that $h_{0}$ be greater than $h_{\eta}$. The behavior of the solution can be understood by looking at $h_{\eta}$ as a function of stratification. When the stratification is large ( $\Gamma$ small), $h_{\eta}$ approaches zero. In this limit, the interface is stiff and its vertical 


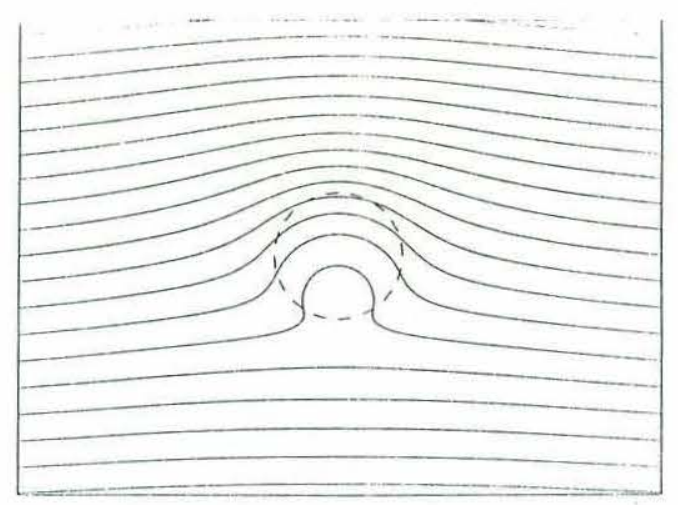

(a)

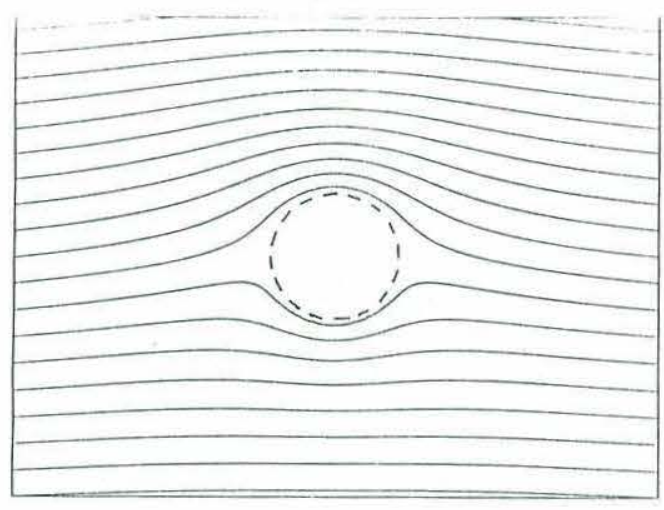

(b)

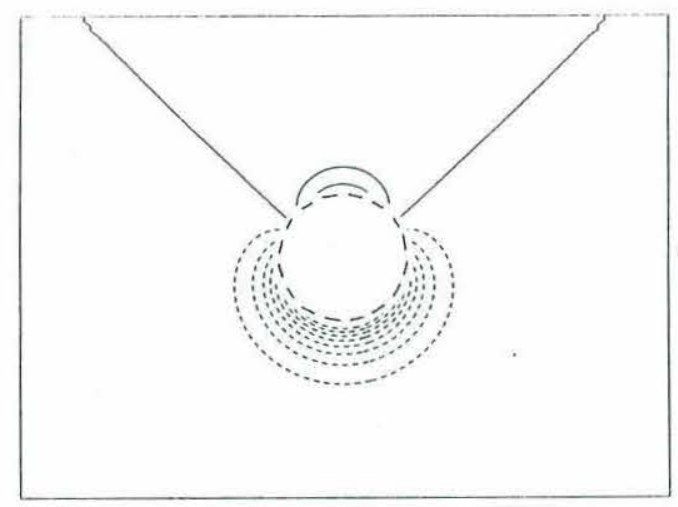

(c)

Figure 3.15: Solution for flow over finite topography with no upstream vertical shear when $h_{0}=1, S=1(\Gamma=2)$, and $d=0.5(\delta=1)$. (a) Upper layer streamlines, (b) lower layer streamlines, and (c) interface height. In this and all the subsequent streamline pictures, the contour interval is 0.4 for the streamlines and 0.1 for the interface height and the flow is from the left to the right. 


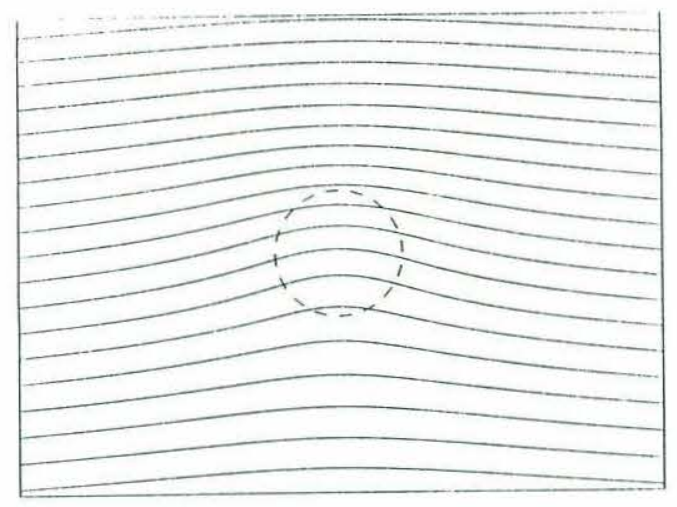

(a)

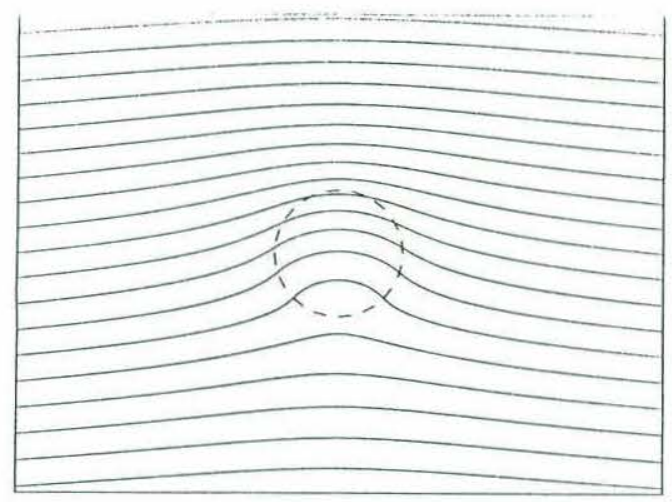

(b)

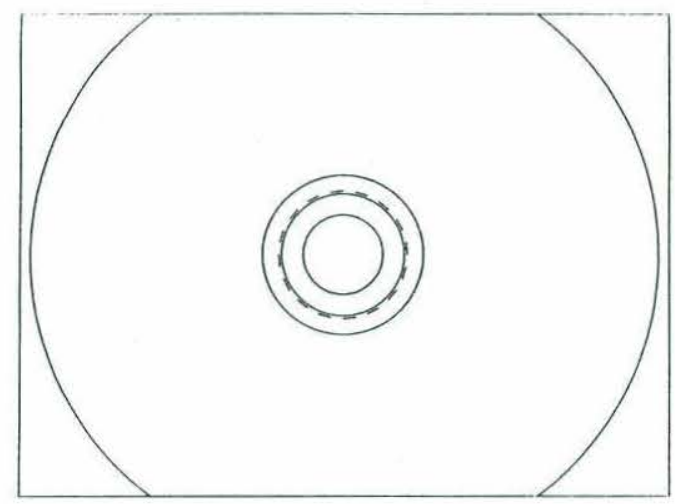

(c)

Figure 3.16: Solution for flow over small topography with no upstream vertical shear using the same parameters as Figure 3.15. (a) Upper layer streamlines, (b) lower layer streamlines, and (c) interface height. 


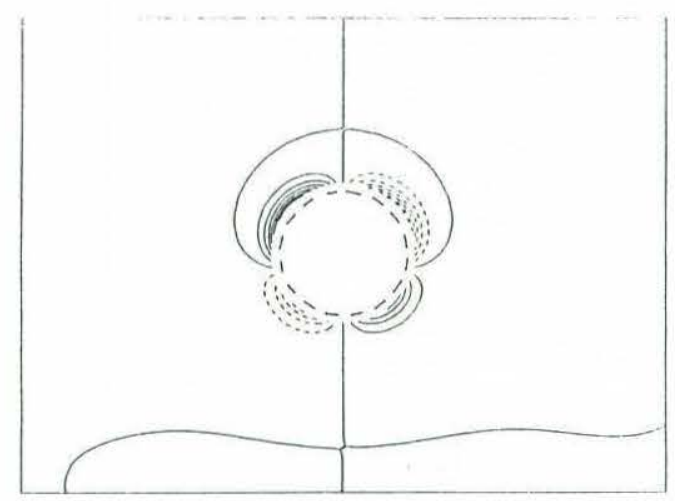

(a)

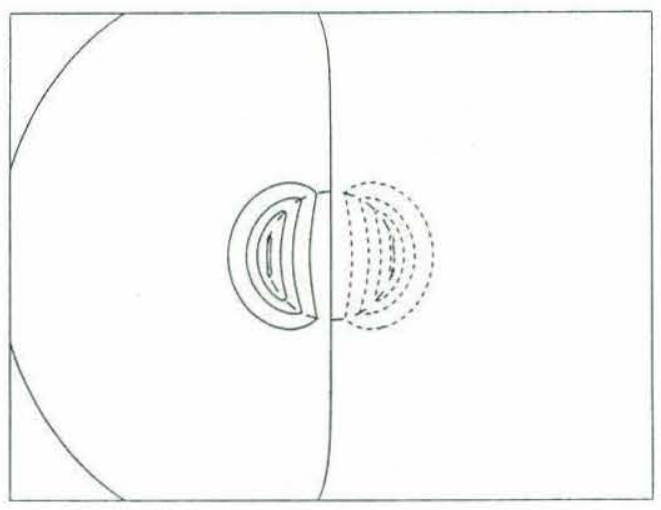

(b)

Figure 3.17: Vertical velocity at the interface in the upper layer using the same parameters as Figure 3.15. (a) Finite depth model, (b) small topography model. 


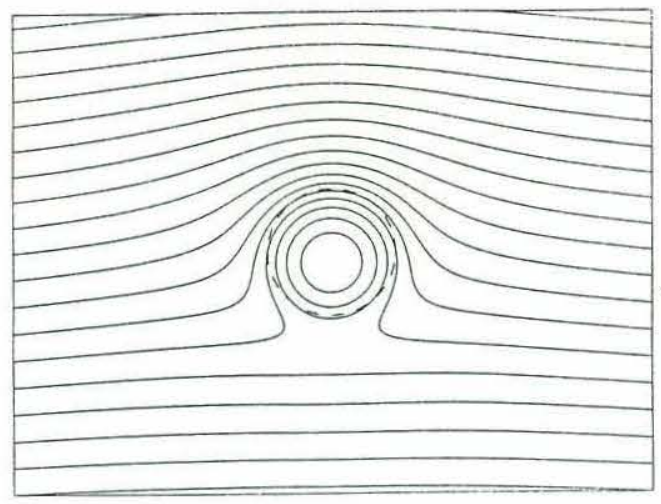

(a)

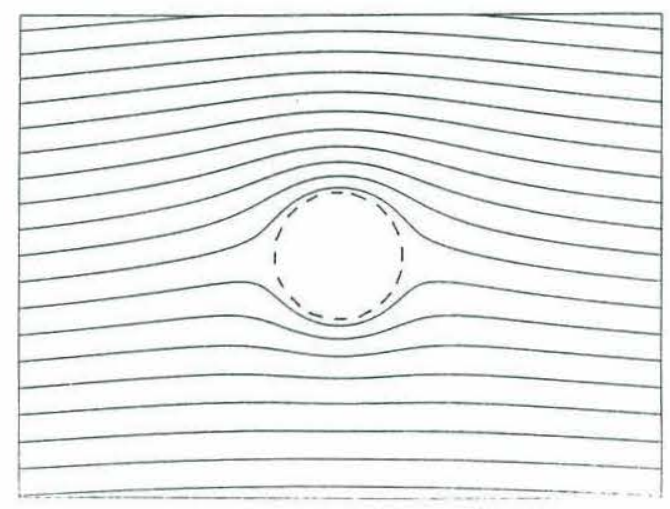

(b)

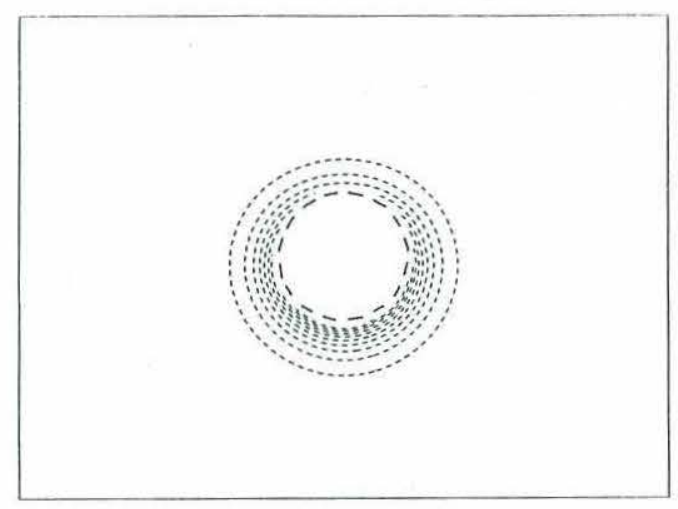

(c)

Figure 3.18: Solution for flow over finite topography with no upstream vertical shear when $h_{0}=1, S=1(\Gamma=2)$, and $d=0.833(\delta=0.2)$. (a) Upper layer streamlines, (b) lower layer streamlines, and (c) interface height. 
deflection is limited. When the stratification is weak ( $\Gamma$ large), the fluid responds barotropically and $h_{\eta} / \epsilon$ approaches $4 \delta /(1+2 \sqrt{\delta})$. The critical height is a monotonically increasing function of stratification, and it is in general larger for smaller values of $d$ (Figure 3.19). We see here that the in the limit of weak stratification $h_{\eta}$ approaches a finite number which depends on $\delta$, or equivalently the upper layer depth. This is consistent with the notion that the validity of the model depends critically on how deep the upper layer is relative to the bottom trapping scale in the upper layer. When the solution violates the critierion, we must use a different model to describe the flow.

There is a critical topographic height above which closed streamlines in the upper layer occur. This critical height gives an indication at what point trapped fluid is expected over the topography and closed streamlines form. This can be found from the solution $3.32,3.33$ and 3.34 , and is given by

$$
\frac{h_{c r i t}}{\epsilon}=\frac{4(1-d)}{\left(2+d \Gamma K_{0}(\Gamma) K_{1}(\Gamma)\right)} .
$$

For strong stratification, $h_{c r i t}$ approaches $2 \epsilon(1-d)$. This is the same as the critical height for a one-layer fluid with relative depth $(1-d)$. When the stratification is strong, the two layers are uncoupled, and the upper layer acts as a one layer fluid independent of the lower layer; the interface is effectively a rigid bottom. When the stratification is weak, the critical height goes to zero; the two layers are strongly coupled, and the lower layer fluid is required to go around the topography. The fluid is responding barotropically, and some of the upper layer also goes around rather than over the topography, which results in a lower critical height.

We can compare the $h_{c r i t}$ to the the critical height above which closed streamlines occur in the two-layer model for flow over small topography. We find the critical height in the lower layer using McCartney's (1975) solution and it is given by

$$
\frac{h_{s 2}}{\epsilon}=\frac{d}{\frac{1}{2} d+(1-d) I_{1}(\Gamma) K_{1}(\Gamma)} .
$$




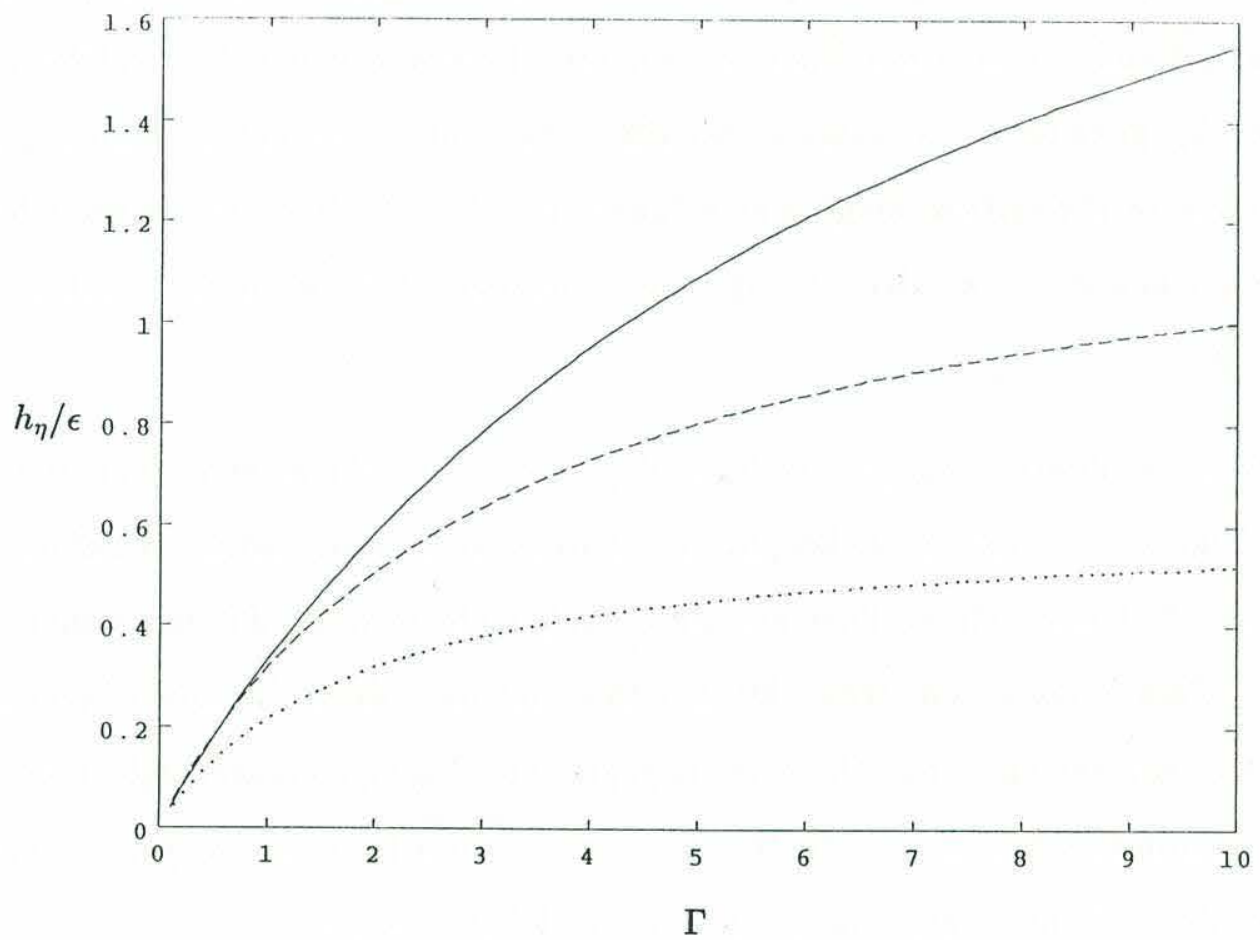

Figure 3.19: Critical height $h_{\eta} / \epsilon$ as a function of $\Gamma$, for $d=0.25$ (solid line), $d=0.5$ (dashed line), and $d=0.75$ (dotted line). 
We find the critical height in the upper layer $h_{s 1}$ numerically.

For strong stratification, $h_{s 2}$ goes to $2 \epsilon d$ which is the same as a one-layer flow with depth $d$, and the interface is effectively a rigid lid. When the stratification is weak, $h_{s 2}$ approaches $2 \epsilon$, which is the one-layer critical height with depth 1 . Thus $h_{s 2}$ decreases with decreasing stratification, opposite to the behavior of $h_{c r i t}$. However, the dependence of $h_{s 1}$ on $\Gamma$ is in the same sense as $h_{c r i t}$. When $\Gamma$ is small, $h_{s 1}$ becomes infinite. The upper layer flow is unperturbed by the topography since the two-layers are uncoupled and closed streamlines never occur. However, when the stratification is weak, then $h_{s 1}$ goes to $2 \epsilon$, the same as $h_{s 2}$ since the fluid is responding barotropically. A comparison of the critical heights as a function of the stratification is done for the two models when $\delta=1$ so that the symmetry between the two models can be seen (Figure 3.20).

The dependence of $h_{\text {crit }}$ of the finite depth model on the relative layer depths is shown in Figures 3.19 and 3.21, keeping the baroclinic Rossby radius of deformation $\left(\Gamma^{-1}\right)$ constant. Closed streamlines are more likely to form when $d$ is large than when $d$ is small. This is consistent with the fact that not only does the lower layer depth increase with increasing $d$, but the total topographic height increases with increasing $d$. $h_{\text {crit }}$ is minimal when $d=1$. In this limit, the bump becomes very tall since the lower layer depth is maximum, and more trapped fluid results.

Huppert and Bryan (1976) calculated a similar critical height in a point vortex model in which they predicted when the fluid that originated over the topography in an initial value problem would remain trapped over the topography and when it would be shed downstream. In their calculation, as the stratification increases, the fluid which originates over the topography is less likely to be trapped. Since their model is a quasi-geostrophic model, their critical height is similar to the critical height $h_{s 2}$ in McCartney's (1975) model. 


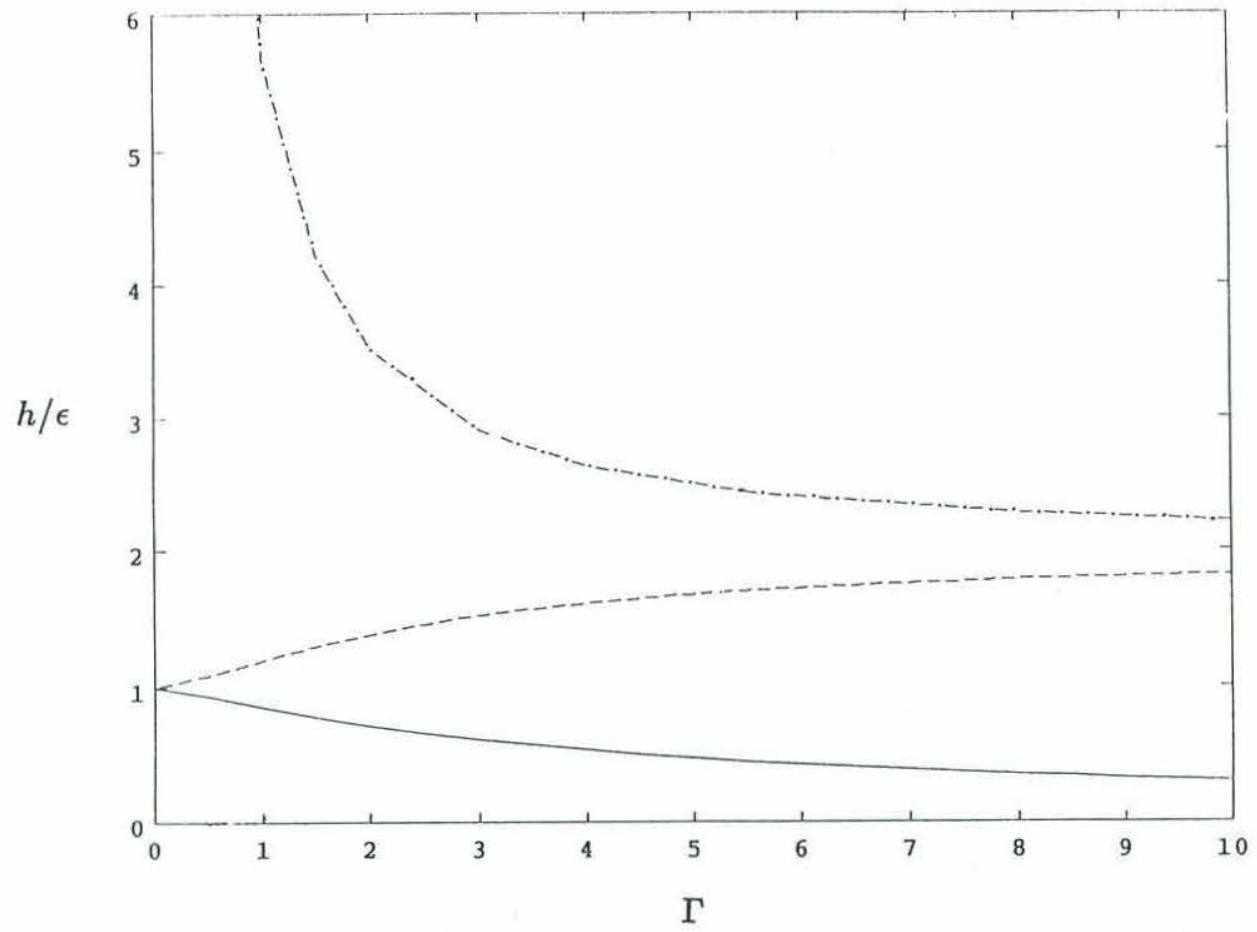

Figure 3.20: Critical heights when $\delta=1$ for both the finite depth topography model and the small topography model. The solid line is $h_{\text {crit }} / \epsilon$, the critical height for closed streamlines to form in the finite depth model. The dashed line is $h_{s 2} / \epsilon$ the critical height for closed streamlines to form in the small topography model in the lower layer. The dotted-dashed line is $h_{s 1} / \epsilon$ the critical height for closed streamlines to form in the upper layer of the small topography model. 


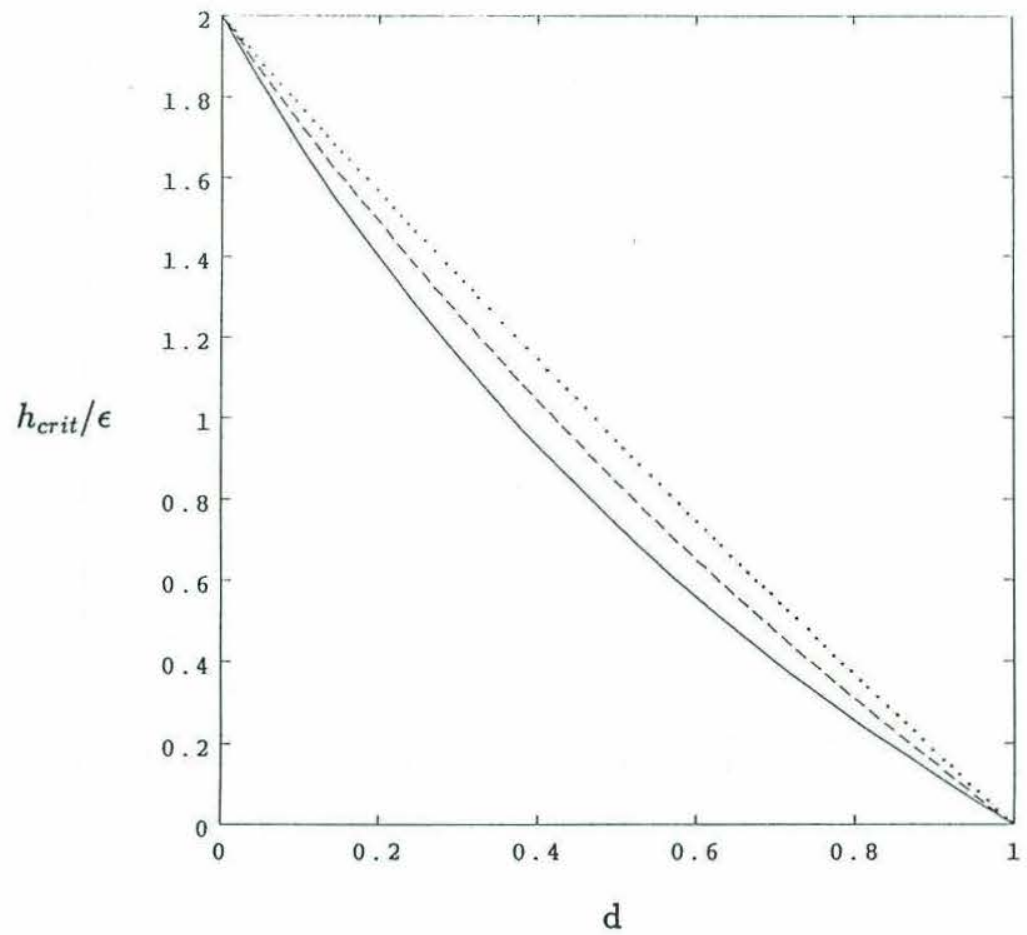

Figure 3.21: Critical height $h_{\text {crit }} / \epsilon$ as a function of $d$ for $\Gamma=1$ (solid line), $\Gamma=0.5$ (dashed line), and $\Gamma=0.25$ (dotted line). 
We have shown in Figures 3.19 and 3.20 that the two critical heights $h_{\eta}$ and $h_{\text {crit }}$ are distinct. This shows that the point at which the model geometry breaks down and fluid parcels go over instead of around the topography in the lower layer is different than the point at which closed streamlines occur. Thus, in a stratified fluid, fluid parcels can go around the topography without a Taylor column being formed.

\subsubsection{Flow on the $f$-plane with vertical shear}

The next most complicated situation to consider is flow on the f-plane with vertical shear. This model provides the first, although simplified, look at the effect of background shear on flow over finite topography. In this case $k_{1}=0$ and $k_{2}^{2}=-\kappa_{2}^{2}=$ $-\Gamma^{2} / U_{1} U_{2}$. Notice however that $k_{3}^{2}=\left(U_{1}-U_{2}\right) / S(1-d) U_{1}$ indicating a wave-like solution when $U_{1}>U_{2}$. Then the solution can be easily written down. For $r>1$ we have

$$
\psi_{1}=a_{1} \ln r+a_{2} K_{0}\left(\kappa_{2} r\right)+a_{3} \frac{\sin \theta}{r}+a_{4} K_{1}\left(\kappa_{2} r\right) \sin \theta-r \sin \theta
$$

and

$$
\psi_{2}=a_{1} \ln r-\delta a_{2} K_{0}\left(\kappa_{2} r\right)+a_{3} \frac{\sin \theta}{r}-\delta a_{4} K_{1}\left(\kappa_{2} r\right) \sin \theta-r \sin \theta
$$

For $r<1$

$$
\psi_{1}=\frac{h_{0}-d}{\epsilon(1-d){k_{3}}^{2}}+b_{1} J_{0}\left(k_{3} r\right)+b_{2} J_{1}\left(k_{3} r\right) \sin \theta .
$$

The stationary waves over the topography come from the effective potential vorticity gradient across streamlines supported by the background vertical shear which maintains a potential vorticity gradient in the exterior. The response in the exterior does not support stationary waves; it is trapped to the topography as evenescent waves since $U_{1}$ and $U_{2}$ have the same sign. The solution over the topography must match to the exterior solution, but only barotropic motions are allowed, and a stationary wave-like solution is forced on the background potential vorticity gradient. Because 
of the wave like character over the topography, the flow is possibly barotropically unstable (e.g. Lorenz, 1972).

To further simplify the problem we look at the limit of the largest vertical shear allowed in the model; no flow in the lower layer. For a consistent solution $\psi_{2}=0$ everywhere. In this case, $k_{3}^{2}=1 / S /(1-d)$ and $U_{1}=\sqrt{1 /(1-d)}$. The exterior solution is written

$$
\psi_{1}=a_{1} \ln r+a_{3} \sin \theta / r-U_{1} r \sin \theta
$$

The coefficients are found by matching across $r=1$ and are given by

$$
\begin{gathered}
a_{1}=-\frac{J_{1}\left(k_{3}\right) q_{a}}{k_{3} J_{0}\left(k_{3}\right)} \\
a_{3}=U_{1} \frac{k_{3} J_{0}\left(k_{3}\right)-2 J_{1}\left(k_{3}\right)}{k_{3} J_{0}\left(k_{3}\right)} \\
b_{1}=\frac{q_{a}}{k_{3}^{2} J_{0}\left(k_{3}\right)}
\end{gathered}
$$

and

$$
b_{3}=-\frac{2 U_{2}}{k_{3} J_{0}\left(k_{3}\right)}
$$

The critical heights can be found as for the barotropic problem. The critical height for closed streamlines to appear is given by

$$
\frac{h_{c r i t}}{\epsilon}=\frac{2(1-d) U_{1}}{J_{1}\left(k_{3}\right)\left[k_{3} J_{0}\left(k_{3}\right)-J_{1}\left(k_{3}\right)\right]} .
$$

At zeros of $J_{1}$ the critical height becomes infinite. At this point, a resonant solution is valid, similar to the one described by McCartney (1975) for eastward flow on the $\beta$ plane. The resonant solution may be an artifact of the abrupt topography, although the wave-like character is not.

The maximum value of $\eta$ is independent of the topographic height $h_{0}$, depending on the background shear and stratification and is

$$
\frac{\eta_{\max }}{\epsilon}=\left|\frac{2 U_{1} J_{1}\left(k_{3}\right)}{k_{3} J_{0}\left(k_{3}\right) S}\right| .
$$


Once again the resonance can be seen. When $k_{3}$ is a zero of $J_{1}$ then this critical height goes to zero. When it is a zero of $J_{0}$, the critical height becomes infinite. When the critical height for closed streamlines $\left(h_{c r i t}\right)$ is a maximum, the maximum value of $\eta$ is zero.

This same kind of behavior can be seen for the more general case of flow with shear. We consider only the oceanographic relevant parameter range where $\left|U_{1}\right|>\left|U_{2}\right|$. Thus, $1<U_{1}^{2}<1 /(1-d)$. Figure 3.22 shows $h_{\eta}$ as a function of $U_{1}$ for a given value of $d$ and various values of $\Gamma$. When $\Gamma$ is small, the critical height becomes infinite for some value of $U_{1}$, so it is not shown. In general, the critical height decreases with increasing $\Gamma$ as was seen in the barotropic problem. The critical height increases with increasing shear. The interface tends to be high in the north as in the barotropic problem. The addition of vertical shear adds additional north-south tilt to the interface from the background flow. What results is that the flow with vertical shear is more likely to go over the topography than flow without (Figure 3.23).

\subsubsection{Westward flow on the $\beta$-plane}

As outlined by McCartney (1975), when the background velocity in both layers is to the west, both of the Rossby waves are evanescent, and $\beta$-plane solutions are qualitatively similar to the f-plane solution. The solution is trapped closer to the topography, just as in the one-layer solution on the $\beta$-plane for westward flow discussed in Chapter 2. The flow with a finite value of $b$ is shown (Figure 3.24) for the same value of the parameters as in Figure 3.15. Note that for barotropic flow, the response over the topography is not wave-like, since the background potential vorticity gradient is simply $\beta y$. Also, less fluid is trapped over the topography than flow on the f-plane. 


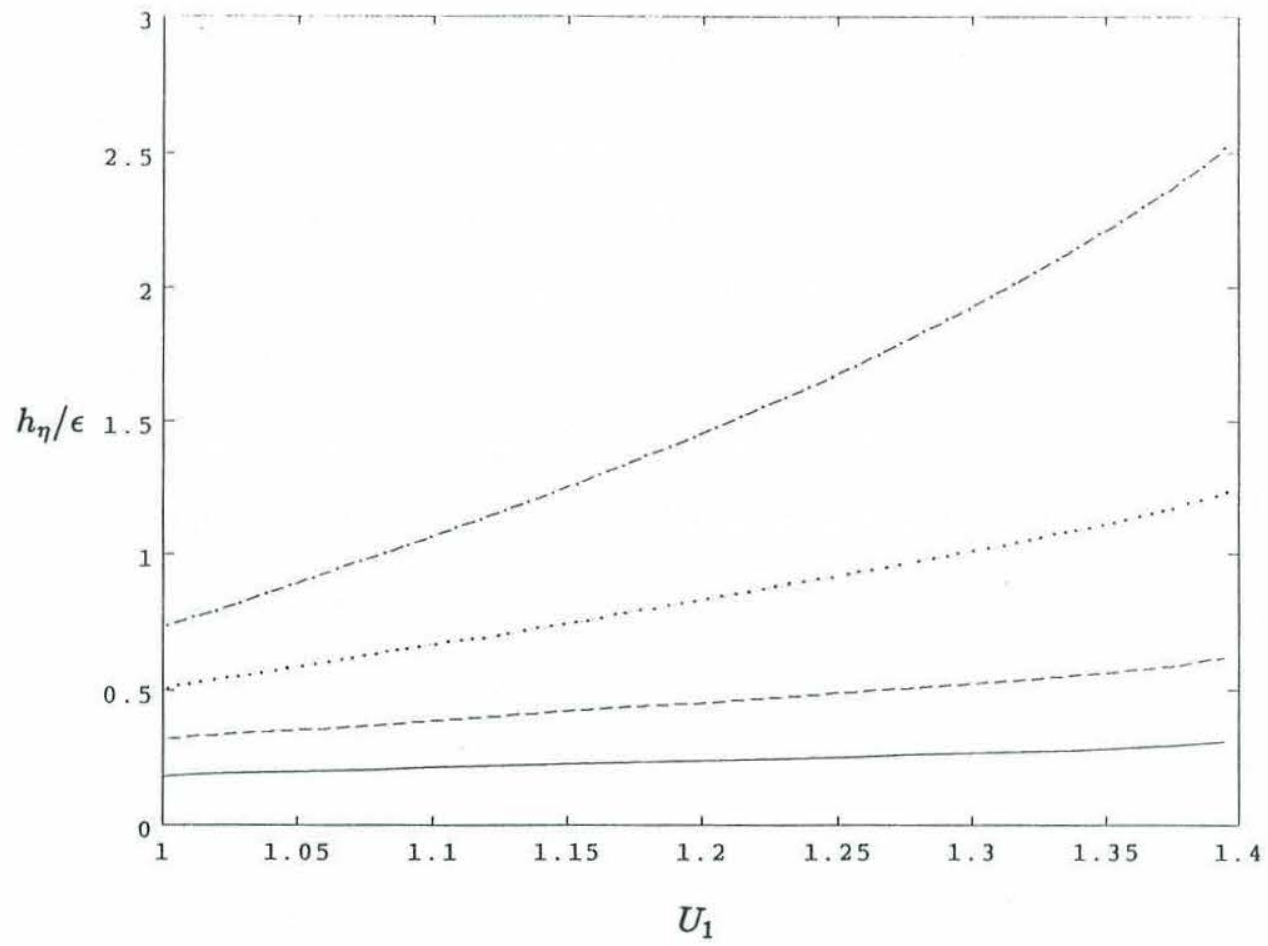

Figure 3.22: Critical height $h_{\eta}$ as a function of $U_{1}$ for flow with shear for $\Gamma=4$ (solid line),$\Gamma=2$ (dashed line), $\Gamma=1$ (dotted line), and $\Gamma=0.5$ (dotted-dashed line). 


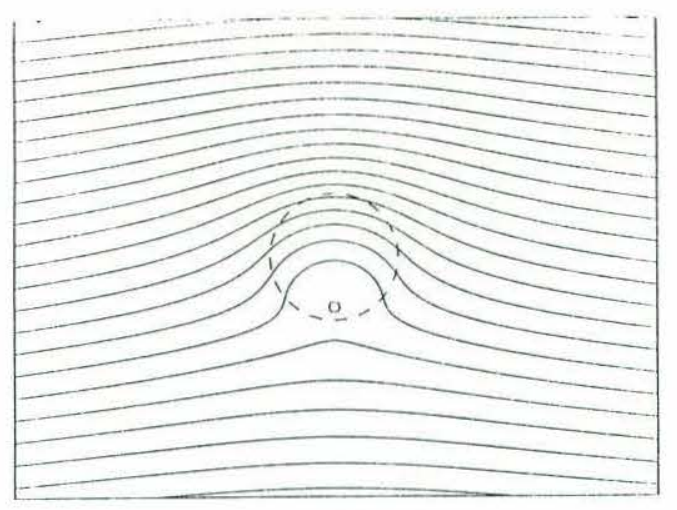

(a)

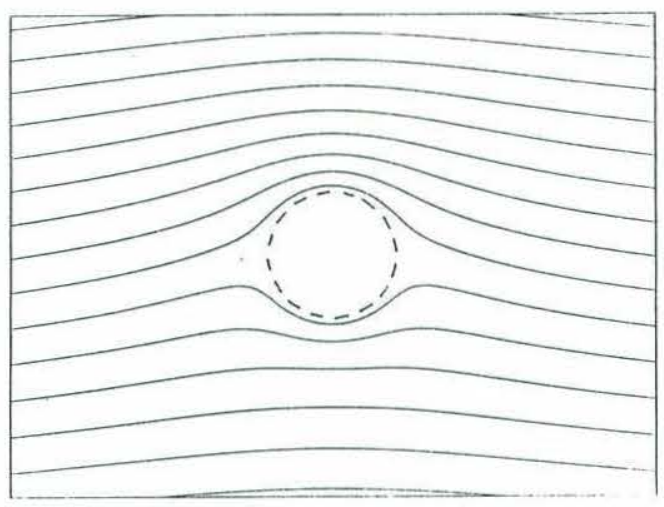

(b)

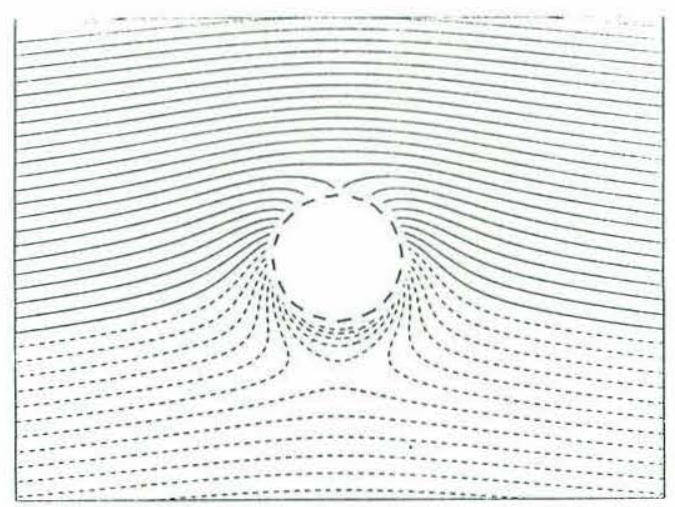

(c)

Figure 3.23: Solution for flow over finite topography with upstream vertical shear, $U_{1}=1.2$, when $h_{0}=1, S=1$ and $d=0.5$. (a) Upper layer streamlines, (b) lower layer streamlines, and (c) interface height. 


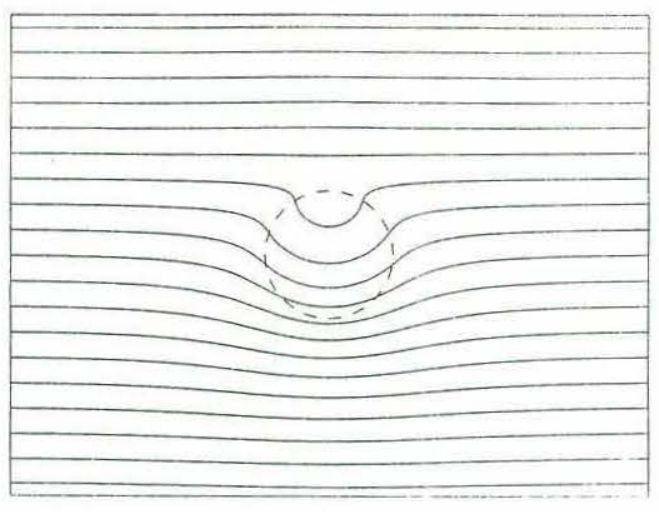

(a)

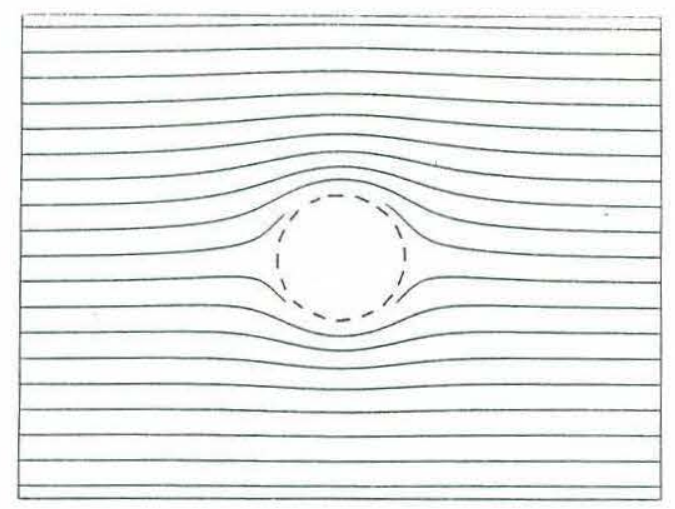

(b)

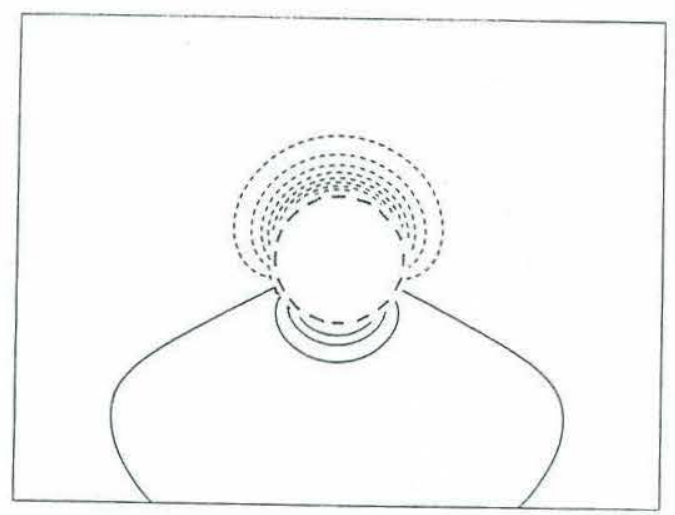

(c)

Figure 3.24: Solution for westward flow over finite topography on the $\beta$-plane when $h_{0}=1, S=1$ and $d=0.5$ and $b=0.5$. (a) Upper layer streamlines, (b) lower layer streamlines, and (c) interface height. The flow is from the right to the left. 
The difference in the two solutions of flow over small topography and finite topography is once again given by the difference in the symmetries of the problem. In particular, for flow over small topography, the deviation from the background flow tends to be isolated on the northern portion of the topography for moderate values of $b$, whereas for finite topography this effect is moderated by the coupling to the lower layer in which the flow is forced to go around the topography. The effect of $\beta$ is to require larger topography for closed streamlines to form, as was true in the one layer model discussed in Section 3.3. Because the effect of the topography is moderated by $\beta$, it takes more coupling between the layers to make the interface go over instead of into the topography.

\subsubsection{Eastward flow on the $\beta$-plane}

McCartney (1975) showed that depending on the relative velocity of the flow in the upper and in the lower layers there can exist either two evanescent waves, one evanescent wave and one radiating wave, or two radiating waves. Because we have already discussed the effects of shear, we concentrate on barotropic eastward flow. The solution in which there is one radiating and one evanescent wave is calculated, which can be compared with the solutions found by McCartney (1975) for flow over small topography. The flow in the upper layer actually looks similar to the solution calculated for the flow in shallow water; the symmetry requires both symmetric and asymmetric stationary waves to be formed which are forced by the application of the boundary conditions in the lower layer (Figure 3.25). More fluid is trapped over the topography than in the westward flowing solution.

There are still situations when the waves resonate at the zeros of the appropriate Bessel functions. These will not be discussed further, but the reader is referred to McCartney (1975) for discussion of the resonant solution in one layer. 


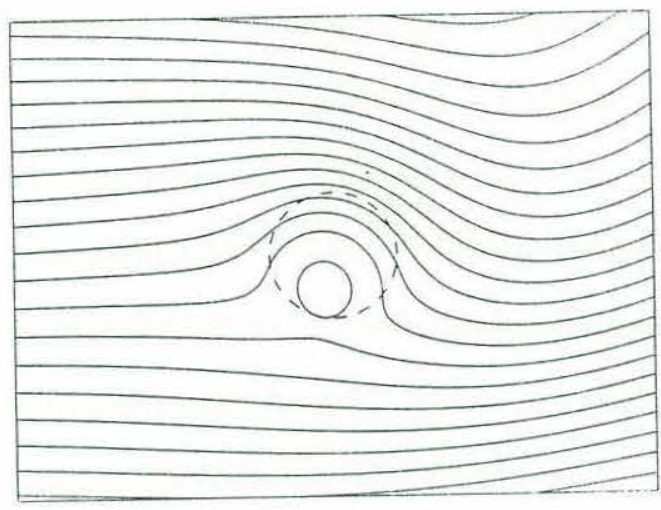

(a)

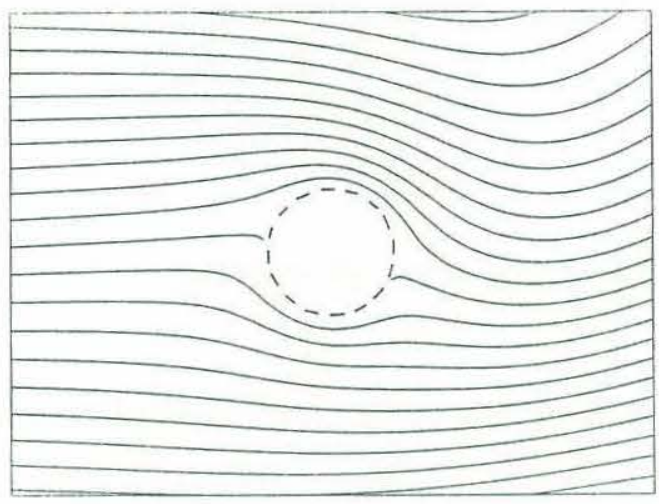

(b)

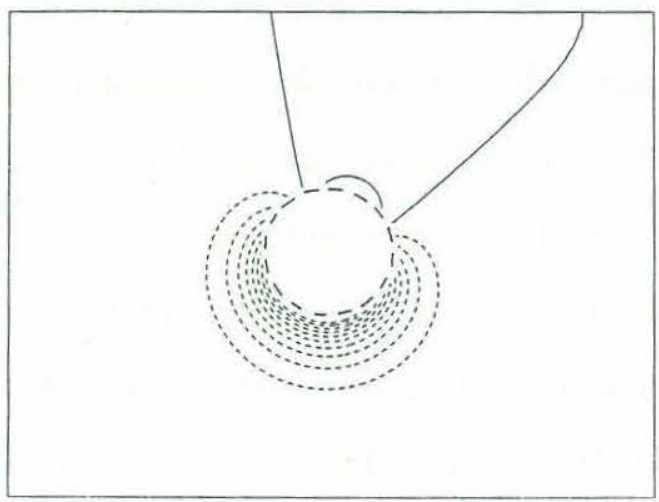

(c)

Figure 3.25: Solution for eastward flow over finite topography on the $\beta$-plane using the same parameters as Figure 3.24. (a) Upper layer streamlines, (b) lower layer streamlines, and (c) interface height. The flow is from the left to the right. 


\subsubsection{Summary: flow over finite topography in a two-layer model}

We have developed a model in which we are able to study flow in a two layer fluid when the interface intersects a boundary. In particular, under the quasi-geostrophic approximation the steady non-linear problem reduces to a linear set of partial differential equations since the interface displacement is simply proportional to the difference between the upper layer and lower layer stream functions. Since the layer depths are constant within an order Rossby number amount, the relative simplicity of the resulting system allows the combined effects of stratification and flow over finite topography to be considered in detail.

We calculated two types of critical heights from this model. The critical height above which closed streamline form $\left(h_{\text {crit }}\right)$ was calculated. Above this height, fluid is trapped over the topography. More fluid is trapped within closed streamlines when the stratification is weak than when it is strong. When closed streamlines form, the solutions found are valid for time scales short compared to the spin up time $\tau$. We also calculated a critical height below which the interface is higher than the topography and the model is no longer physically consistent. This critical height is large when the stratification is weak.

The limit that the topography must be taller than the interface height $\left(h_{\eta}\right)$ is interesting because past solutions of quasi-geostrophic flow in a stratified fluid over finite topography have broken down as soon as the isopycnals intersected the topography. The model presented in this chapter breaks down when the isopycnal no longer intersects the topography; therefore we have extended our understanding of stratified flow over finite topography by considering a new physical regime. We show that as the stratification decreases, fluid tends to go more easily over the topography, the same result as Schar and Davies (1988). The model also predicts that the interface 
is depressed on the southern portion of the topography, instead of uniformly raised over the topography as traditional quasi-geostrophic models predict. There is an anticyclonic perturbation in the upper layer which coupled with the requirement that there is no circulation in the lower layer causes the interface to be low near the topography; the north-south asymmetry is due to the change in symmetry of the solution resulting from application of the boundary conditions in the lower layer. Because of the simplicity of this model relative to the continuously stratified models, the effects of vertical shear in the background flow as well as the effects of $\beta$ can be seen. The interface goes more easily over the topography as the shear is increased.

Although we do not expect to be able to make detailed predictions from this two-layer model, it may help in the interpretation of primitive equation models of stratified flow over topography. Since the model is quasi-geostrophic, it does require that the flow speed be quite small for the solutions to be relevant. Most importantly, we have shown how a quasi-geostrophic model in which the boundary conditions at the topography are applied on the horizontal velocity is fundamentally different than a model in which the boundary condition is applied on the vertical velocity. Quasi-geostrophic theory is equally valid for this model since the flow remains quasihorizontal. The solutions are more relevant than that found by Buzzi and Speranza (1979) where they considered an obstacle with finite vertical cross section, but small horizontal cross section. They also apply an arbitrary condition on the vertical structure of the stream function at the wall. In this model, we combine the effects by considering an obstacle with both finite vertical and horizontal cross sections.

There is one issue which has not been resolved; we showed that in the island limit, the barotropic finite depth model has finite circulation around the topography, but we require that the flow in the lower layer of the two-layer model has no circulation. There are several ways to try and resolve this difference. First, friction can be invoked to argue that in the barotropic model, the circulation would be dissipated 
given enough time. A connection however, would have to be made to solutions of the form discussed in Chapter 2. In the limit of finite topography, Ingersoll's (1969) solution has finite circulation required because the velocity vanishes pointwise on the boundary of the Taylor column which is slightly inside the edge of the topography in the limit that it becomes tall. But there is a strong shear layer from the small crescent of fluid forced onto the topography and acquiring strong anticyclonic vorticity. Thus there is finite negative circulation. This result may be different in a non-quasi-geostrophic model. In the presence of bottom friction, we do not expect that the tangential velocity should vanish at the boundary of the topography in the two-layer model. We can also consider the problem as the steady state of an initial value problem in which there is no circulation initially and the flow develops inviscidly so that no circulation is ever induced (as long as the lower layer does not overflow the topography). In the next chapter, we show an example of the initial value problem in the finite depth barotropic model in which all of the fluid which originated over the topography remains trapped near the topography. This suggests that when the topography is quite tall in the finite depth model, no circulation would be induced in the inviscid initial value problem.

The consequence of the requirement that the circulation be zero in the lower layer in the two-layer model is that the displacement of the interface tends to be negative near the topography. If the circulation were non-zero in the lower layer, then the interface deflection would be different and given strong enough circulation in the lower layer, the interface deflection could change sign. In continuously stratified primitive equation models, isopycnals tend to rise near the topography. This discrepancy could be because in our model the density change is limited to one place in the vertical, because the flow remains nearly horizontal due to the quasi-geostrophic requirement or because the topography is a right circular cylinder and has vertical faces. In a primitive equation model over smoothly varying topography, vorticity is generated near the topography through vortex stretching whenever fluid parcels move 
up the slope. Thus, in a more realistic model, there is probably not a lower layer in which the circulation is exactly zero. We maintain that our choice of circulation is the most physical choice to make for understanding flow over finite topography in the simple model that we constructed here.

Both of the models described in this chapter are more relevant to the ocean than the atmosphere because the topography considered is tall and can be approximated by a fluid with a rigid lid. In the ocean however, the tallest seamounts do not have very large horizontal extent so in general we expect that the effect of $\beta$ would be somewhat limited. The second model of flow over finite topography in a two-layer fluid is limited by the restrictive geometry. We can think of this just as a first step in understanding of flow over finite topography in a stratified fluid. Because the size of the topography and the relative layer depths are directly related, the most general situations cannot be considered here. 


\title{
Chapter 4
}

\section{Time dependent solutions}

\subsection{Introduction and background}

\begin{abstract}
Although steady solutions are useful for isolating dynamical processes at work in the ocean, oceanic flow has a large time-dependent component especially at the synoptic scale. The models described in the previous chapters have shown some solutions of flow over topography which has reached a steady state. In this chapter we explore several time-dependent models of flow over isolated topography on the f-plane by considering the start up problem of flow impinging on topography and looking at the generation of eddies through the movement of fluid on and off the topography. Circulation is generated in the eddies by topographic stretching. We consider only flow on the $\mathrm{f}$-plane. In Chapter 3 we considered situations in which the $\beta$-effect is important. Here we take the approach of considering the simplest flow situation by only considering solutions on the f-plane. By doing this, we are isolating the process of generation of vorticity through vortex stretching, and ignore the effect of planetary potential vorticity gradients. If we were to include the $\beta$ effect, Rossby waves could be generated at the topography, and the shed eddy could be influenced by the radiation of Rossby waves (Flierl, 1984).
\end{abstract}


By restricting ourselves to flow on the f-plane, we can use the method of contour dynamics. We focus on a new parameter regime for simple time-dependent numerical models, flow over finite topography and in the process develop a modification to contour dynamics so that the matching conditions at the boundary of the topography can be handled. As for the steady models that are discussed in Chapter 3, there are order one differences between traditional quasi-geostrophic models and models of flow over finite topography.

A hierarchy of time-dependent models on the $\mathrm{f}$-plane is considered. First we review and extend the work of Kozlov (1983), who also used the method of contour dynamics to model time-dependent flow in one layer over a quasi-geostrophic bump. Then, flow over topography of finite height in a barotropic fluid corresponding to the steady solution found in Section 3.3 is studied. We extend Kozlov's model to include the effects of stratification by considering a quasi-geostrophic bump in a two-layer fluid, corresponding to McCartney's (1975) steady solution. Finally, quasigeostrophic flow in two-layers over topography of finite height is modeled, corresponding to the geometry used in Section 3.4. As an introduction to the four models, a side view of each model is shown in Figure 1.1. The geometry and numerical technique used in these models is simple and efficient; a wide range of parameters can be considered at relatively little computational cost. Although these are certainly not the most complete models to date, because we have restricted ourselves to the simple situation of flow on the f-plane with no vertical shear in the background flow, the dynamical processes at work in the models are relatively transparent. In the process of applying contour dynamics to the finite depth models, the method is modified such that we extend the range of problems that can be considered with contour dynamics.

Modeling studies of time-dependent motions of flow over topography have been limited primarily to flow over low features. The first such study of eddy shedding consisted of the modeling of flow over a small bump $(400 \mathrm{~m})$ in a continuously 
stratified ocean using the fully non-linear primitive equations (Huppert and Bryan, 1976). Despite the relative complexity of the model, they observed that the flow tended to be in one of two configurations. When the flow is strong enough, a cyclonic warm core eddy is shed downstream of the topography. When the flow is weaker, the eddy remains trapped near the topography. This model included a horizontal eddy viscosity coefficient, and was doubly periodic, so that it could only be run until the shed eddy came back into the domain. An idealized point vortex model suggested when the eddy would be shed and when it would remain trapped and what the path of the shed eddy would be.

James (1980) considered the simpler problem of barotropic quasi-geostrophic flow over an axisymmetric bump using a finite difference model. He showed the time-dependent effects on topographic drag, and found the steady state solution by solving the initial value problem numerically. This simpler model demonstrated the qualitative nature of the flow seen in the more complicated model of Huppert and Bryan (1976). James (1980) found that the drag was initially larger when the fluid which originated over the topography is moved partially off the topography, and has a transient response that oscillates with time. The transient response period increases with decreasing friction. When the eddy is partially trapped, he observed that a patch of positive vorticity spirals onto the hill, and successive pieces of it break away and then coalesce back into the main patch of positive vorticity. Because of the finite difference nature of his model, friction is always important; the model is not numerically stable when the friction is zero. The drag and lift are of similar magnitude during the transient regime.

Verron and LeProvost (1985) also studied the quasi-geostrophic equations, but included the effect of $\beta$. They observed the two regimes that Huppert and Bryan (1976) described: eddy shedding and eddy trapping. Several simulations of flow on the $\beta$-plane were made. Westward flow on the $\beta$-plane looks quite similar to flow on 
the f-plane. When the flow is eastward, Rossby waves are generated downstream of the obstacle, changing the character of the flow. The simulations are performed in a channel, and very quickly the Rossby waves are affected by the walls to the north and south. Verron (1986) considered time-dependent incident flow over topography. In this case, oscillatory incident flow is applied, and through the action of friction, successive anticyclonic eddies are shed off of the topography.

The models described above have three things in common. First, they were all performed in a confined or periodic domain. This restricts the studies to times short enough such that the shed eddy does not return into the domain from upstream, and requires that the flow is not effected by the boundary domains. Second, they all modeled flow over small topography. Finally, friction, whether numerical or otherwise, plays an important role in the dynamics of the flow. In order to explore the effect of all these limitations, we use a contour dynamics model that is performed in an infinite domain and is inviscid, and modify the method so that some aspects of flow over finite topography can be addressed. We hope that the simple (and hence easily understood) models presented in this chapter will help with the interpretation of the next generation of models as well as observations of the ocean.

\subsection{Flow in one layer over small topography}

The method of contour dynamics is well suited for use in the study of the eddy shedding process. It has two advantages over conventional models. First, it is inviscid, and second, the flow is found over an infinite domain so that if the eddy is shed downstream, it does not reflect off either the boundary of the domain or return to the domain through the effects of periodic boundary conditions. The drawback is that we are restricted to the study of flow over topography that is piecewise constant. Before showing how contour dynamics can be used in the study of flow over topography, we 
first describe the technique in general. This technique has been used previously in the study of vortex dynamics ( Polvani, 1988; and Zabusky et. al., 1979).

\subsubsection{Numerical method}

In a barotropic, two dimensional fluid, the vorticity is advected by the stream function. The evolution of the fluid can be described by the evolution of the vorticity which is governed by

$$
\frac{\partial \nabla^{2} \psi}{\partial t}+J\left(\psi, \nabla^{2} \psi\right)=0
$$

If the vorticity $\nabla^{2} \psi$ is known everywhere and the boundary conditions are known, we can calculate the stream function using a Green's function,

$$
\psi(x, y)=\iint q(\xi, \eta) G\left(x, x^{\prime}, y, y^{\prime}\right) d x^{\prime} d y^{\prime}+\text { boundary contributions }
$$

where $q=\nabla^{2} \psi$. For Laplace's equation in a domain with no boundaries, the Green's function takes the simple form

$$
G\left(x, x^{\prime}, y, y^{\prime}\right)=\frac{1}{2 \pi} \ln R,
$$

where $\left.R=\left[\left(x-x^{\prime}\right)^{2}+\left(y-y^{\prime}\right)^{2}\right)\right]^{1 / 2}$. We consider the problem where the vorticity $q$ is piecewise constant so that it can be taken out of the integral. In order to calculate the velocity at any point, we differentiate 4.2 with respect to $x$ and $y$, invoke the symmetry of the Green's function, and use Green's theorem to obtain

$$
(u, v)=\left(-\partial_{y} \psi, \partial_{x} \psi\right)=q \oint_{\partial D} G(R)\left(d x^{\prime}, d y^{\prime}\right) .
$$

The velocity at any point is found by simply doing a contour integral. In order to find the evolution of the flow field, the evolution of $\partial D$ must be known, and it can be found by calculating the velocity on $\partial D$.

The advantage of this method is that it reduces the problem of solving for the non-linear evolution of the field to that of evaluating 4.4 at each time step. 
Once the velocity on $\partial D$ is known, then it can be stepped forward in time to find the new location of the contour. The implementation of this technique used in this thesis is described in Polvani (1988). At each time-step, the boundary of the region of constant relative vorticity is stepped forward via Runge Kutta integration. The Green's function is singular on the contour, but the singularity can be handled by using the method Polvani (1988) outlines in his Appendix B. This method has been used throughout this chapter for all of the contour integrals. The basic contour dynamics computer code used in this chapter was developed by Meacham (1990).

As the contour deforms with time, the distances between the points on the contour change, and an adjustment in the spacing of the points on the contour must be made to accurately carry the calculation forward in time. For the calculations here we applied a point adjustment scheme developed by Meacham (1990). The points are redistributed according to the local rate of curvature. In addition points are added or removed as needed, and when the contour comes back on itself, the contour is pinched off. Only when many (on the order of 10) pinch-offs have occurred is there a significant loss of vorticity (on the order of several percent) in all of the calculations in this chapter.

In order to understand the implementation of this technique for the problem of flow over topography, the simplest problem of barotropic flow in one layer over quasigeostrophic topography is discussed first. This work is a review of the work of Kozlov (1983). For slow flow over small topography, the quasi-geostrophic approximation can be made. In this case, the potential vorticity advected by the flow is given by

$$
q=\nabla^{2} \psi+\frac{h}{\epsilon}
$$

which is the non-dimensionalized potential vorticity equation where $h$ is the nondimensional depth function, and $\epsilon$ is the Rossby number defined by $\epsilon=U / f L$ where $U$ is the velocity scale and $L$ is the length scale of the topography. When the topography 
is piecewise constant, the relative vorticity is piecewise constant and is given by equation 4.5 .

Before the background flow is turned on, there is no flow anywhere, so the quasi-geostrophic potential vorticity is zero away from the topography and is $h_{0} / \epsilon$ over the topography while the relative vorticity is zero everywhere. When we turn on a background flow, the fluid over the topography is pushed off the topography, and is stretched so it gains cyclonic vorticity of size $h_{0} / \epsilon$. Meanwhile, fluid from upstream is pushed over the topography and is squashed, so it gains anticyclonic vorticity of size $-h_{0} / \epsilon$.

There are two regions of non-zero vorticity to consider (Figure 4.1). The region of fluid which originated upstream that moves over the topography (region A) has relative vorticity $-h_{0} / \epsilon$; the region of fluid which originated over the topography that moves off the topography (region C) has relative vorticity $h_{0} / \epsilon$. Region B has no relative vorticity and is composed of fluid parcels which originated over the topography and remain there. This region is the overlap of the circle and the line which delineates the region of fluid which originated over the topography at $t=0$. In order to find the flow everywhere, a contour integral around region A and a contour integral around region $\mathrm{C}$ are done. Instead of evaluating the contours that bound region $\mathrm{A}$ and $\mathrm{C}$ separately, we evaluate the topographic contour (a circle) using potential vorticity $-h_{0} / \epsilon$ and the contour that bounds the fluid that originated over the topography using potential vorticity $h_{0} / \epsilon$ and add the results to take advantage of the cancellation in region B. This method can be used for any arbitrarily shaped region and is not restricted to a circle. The problem that Kozlov (1983) considered was flow over a circular cylinder, where an analytic solution can be found for the contour integral around the circular topography, simplifying the calculation even further.

Once the position of the contours are known, the velocity field can be calculated everywhere. The stream function is the inverse laplacian of the vorticity, and so 


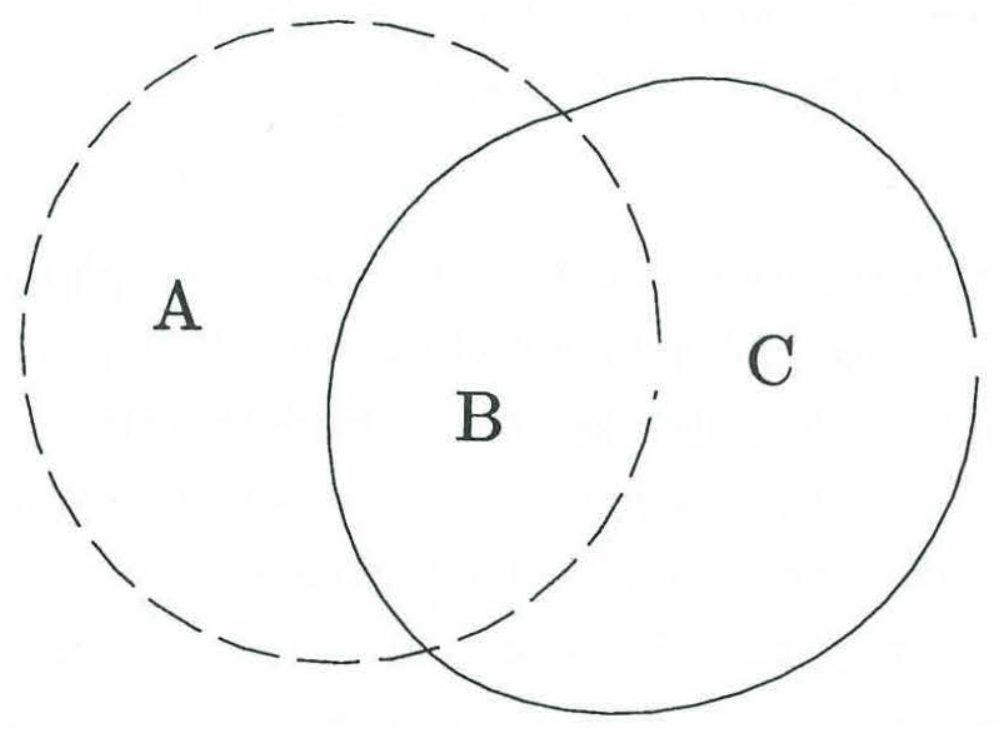

Figure 4.1: Schematic of the different regions of constant relative vorticity. Outside of the two contours, the vorticity is zero. In region $\mathrm{A}$, the vorticity is anticyclonic with value $-h_{0} / \epsilon$, in region $\mathrm{B}$ the vorticity is zero, and in region $\mathrm{C}$ the vorticity is cyclonic with value $h_{0} / \epsilon$. The circular dashed contour delineates the boundary of the topography. The solid contour delineates the boundary of the fluid which originated over the topography. 
is much smoother field than the vorticity field. Even though the vorticity jumps discontinuously on the boundary of the contours, the velocity field does not. The velocity field can be inferred from the positions of the contours at each time step. For instance, in Figure 4.1 the flow is anticyclonic in region A, cyclonic in region $\mathrm{C}$ with a uniform background flow superimposed. Therefore, the flow field that corresponds to the positions of the contours in Figure 4.1 is a dipole field imbedded in a uniform background flow. Examples of the velocity field for various cases will be shown in the next section.

In this chapter we concentrate on the flow field that is induced when uniform flow (with zero potential vorticity) impinges on topography. We have one free parameter, $h_{0}$, and are free to choose the time evolution of the zero vorticity background flow.

\subsubsection{Numerical solutions}

In this section some examples of time-dependent solutions are shown to illustrate the basic dynamics of eddy generation and vortex interaction resulting from flow over topography. There are two basic dynamical regimes. First, when the topogra-

phy is small enough, all of the fluid which originates over the topography is swept downstream by the background flow. When the topography is tall; some of the fluid that originates over the topography remains trapped there because the anticyclonic flow generated over the topography is large enough to overcome the effect of the background flow. In many cases at least some of the fluid that originated over the topography escapes downstream.

We first illustrate (following Kozlov, 1983) complete shedding of the fluid which originates over the topography when we let $h_{0} / \epsilon=1$ (Figure 4.2). The shape of the region of marked fluid which originated over the topography changes from a circle to 
a tear drop shape as it is influenced by the cyclonic circulation over the topography, but it remains coherent. The shed fluid remains close to the $x$ axis as it moves downstream.

When the topography is large, or the flow is weak, some of the fluid remains trapped over the topography, while some is shed downstream (Figure 4.3). This process is caused by the interaction between the region of anti-cyclonic relative vorticity generated over the bump, and the region of cyclonic relative vorticity generated off the topography. After several advection times, the contour has a C-shape. Initially, the fluid which originated over the topography is pushed downstream; it then moves clockwise around the topography in response to the anticyclonic vorticity. The upper part of the contour is advected over the top of the bump, while the lower part is stretched downstream, giving the $\mathrm{C}$-shape. Then, the upper part of the $\mathrm{C}$ is advected to the south and clockwise around the topography while the lower part of the $\mathrm{C}$ is shed downstream. The contour becomes quite complicated, although there is a tendency for the fluid to rotate clockwise around the topography. Eventually the calculation must be stopped because the contours become too complicated. Unlike Kozlov (1983), we proceed to calculate the velocity field near the topography which is determined once the position of the contours is known. This field is shown in Figure 4.4 where we can see how the fluid which originated over the topography is advected by the velocity generated from the vorticity field. The velocity field is smoother than the vorticity field since it is an integral of the vorticity.

As Verron and LeProvost (1985) suggested after the fluid has been moved to the base of the bump (for instance see $t=1$ in Figure 4.3) it appears to be in a state similar to the steady-state solution found by Johnson (1978). However, the flow is progressively altered. Kozlov (1983) showed that Johnson's (1978) solution is unstable to small perturbations so it is not surprising that the fluid does not remain in this state. The velocity field (Figure 4.4a) at $t=1$ shows that in this early state the 

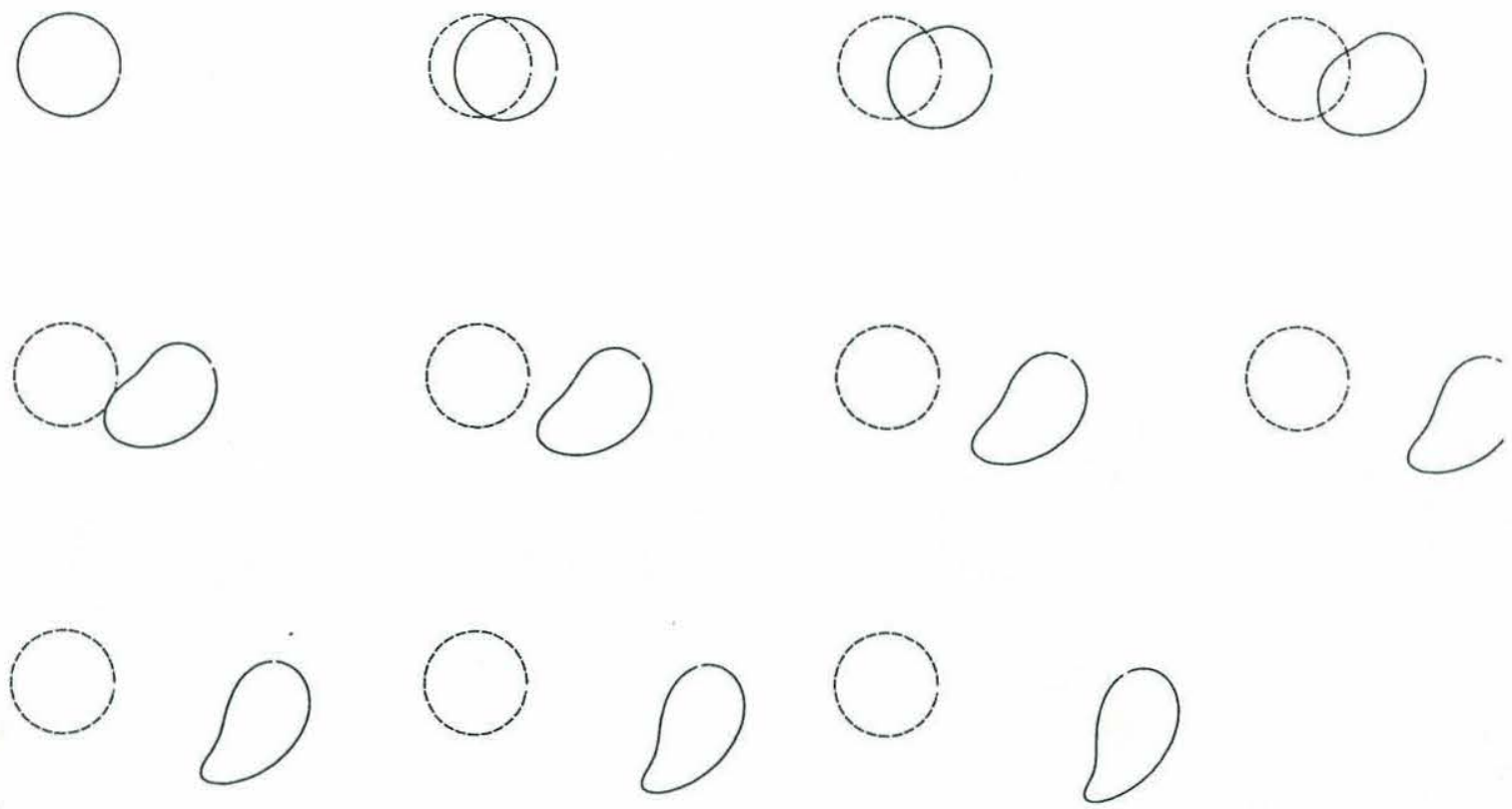

Figure 4.2: Time evolution of the contour for a case when all of the fluid is shed downstream. For this case, $h_{0} / \epsilon=1$ and the flow is turned on abruptly at $t=0$. A snap shot of the contours is taken at $t=0,1, \ldots, 10$ with time increasing to the right and downward. The dashed contour is the boundary of the topography; the solid contour delineates the boundary of the fluid that originated over the topography 

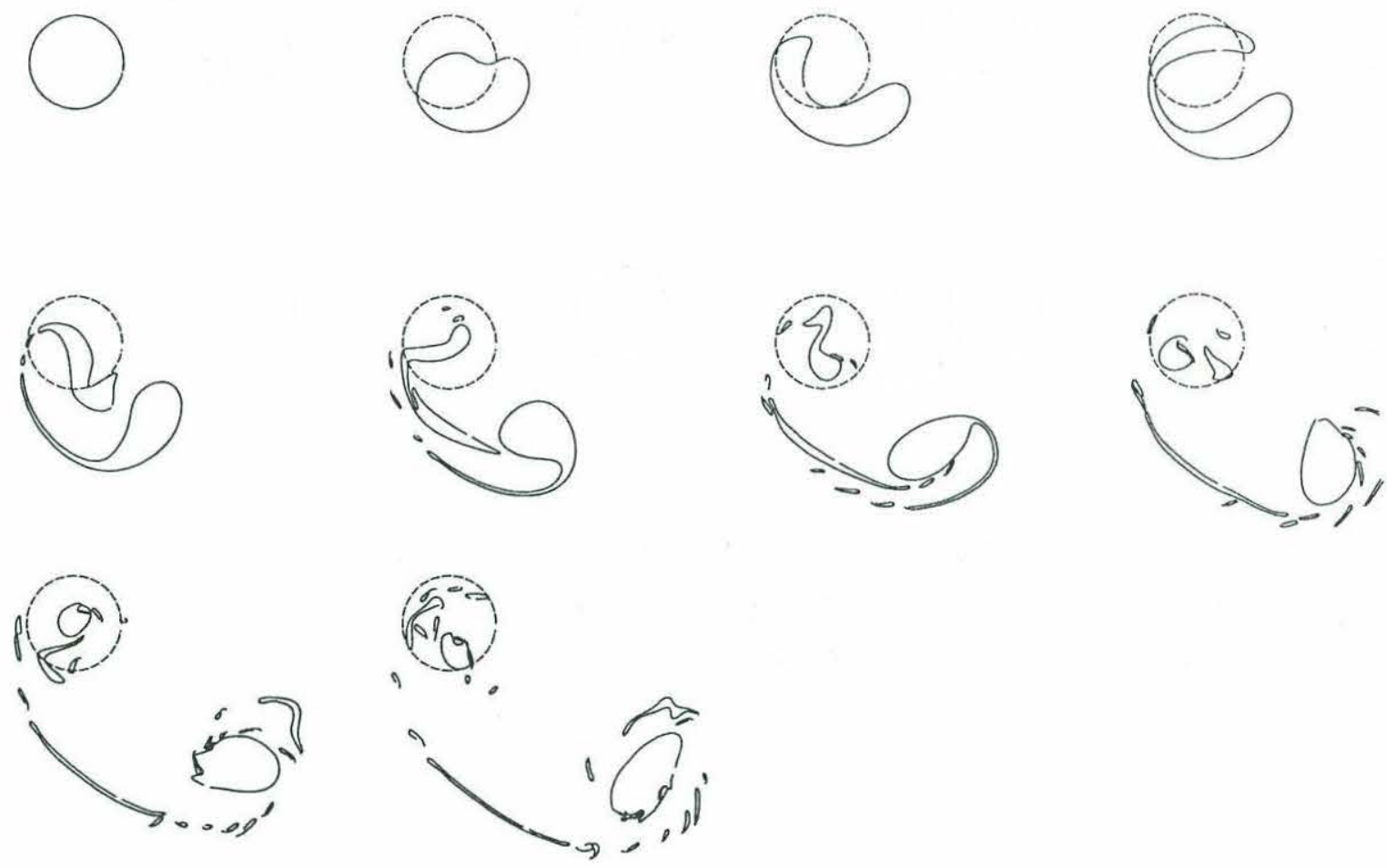

Figure 4.3: Time evolution of the contour for a case when some of the fluid is shed downstream. For this case, $h_{0} / \epsilon=5$ and the flow is turned on abruptly at $t=0$. A snap shot of the contours is taken at $t=0,1, \ldots, 9$. 


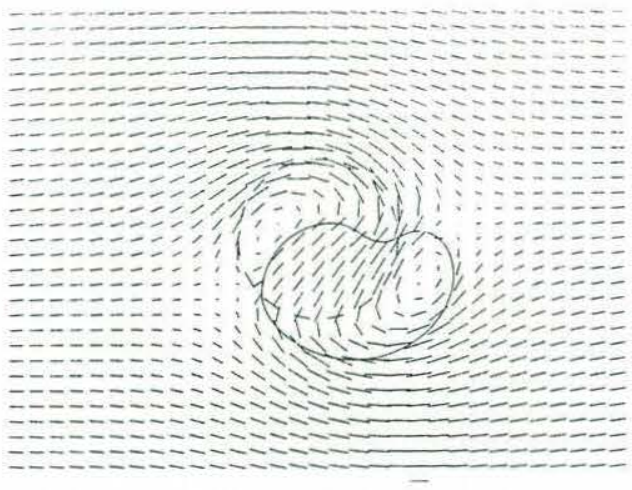

(a)

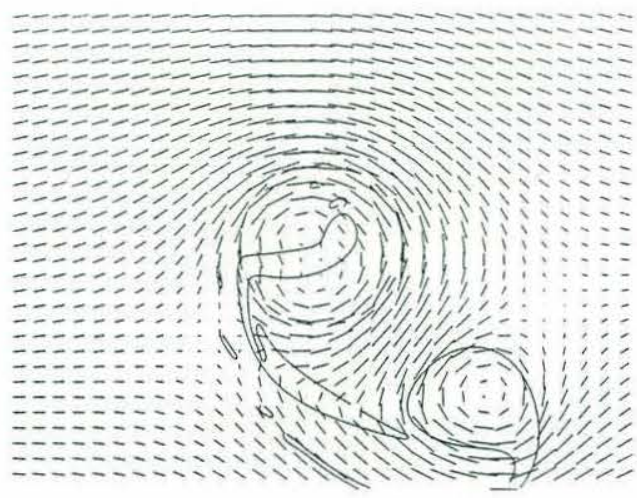

(c)

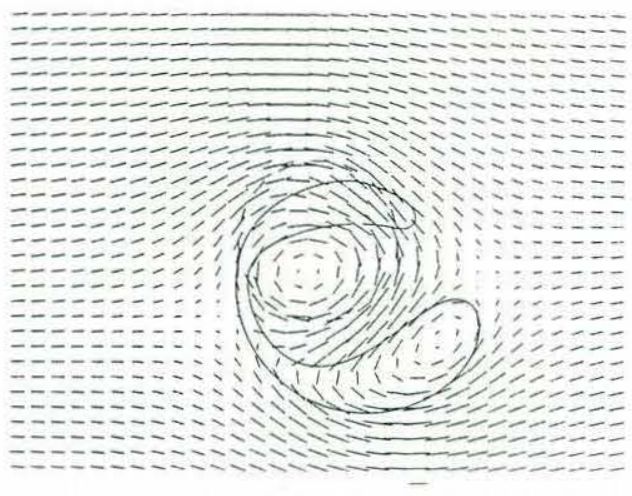

(b)

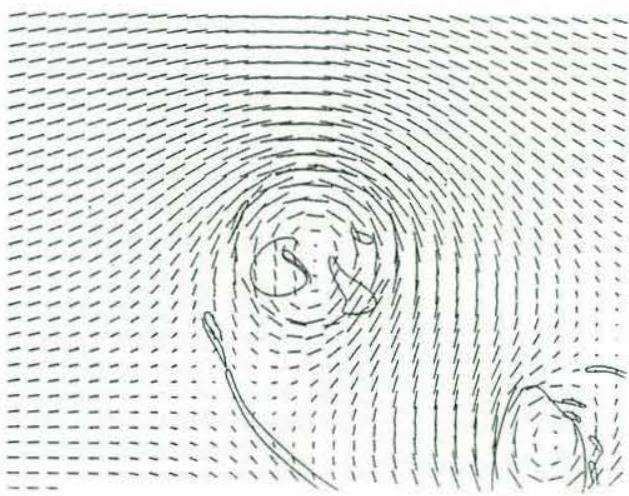

(d)

Figure 4.4: Velocity field for the case shown in Figure 4.3 at (a) $t=1$, (b) $t=3$, (c) $t=5$, and $(\mathrm{d}) t=7$. 
flow field has a dipole structure. Both the eddy that is trapped over the topography and the shed eddy contained closed streamlines as can be seen in Figure 4.4.

When the vortex interaction is strong, as in our example, the cyclonic eddy is wrapped up in the anticyclonic eddy (Figure 4.3). We observe progressive ejection of filaments from the patch of fluid that originated over the topography as the contour is advected clockwise around the topography. We attempt to understand this process to first order as the progression of a topographic Rossby wave around the topography. Linear theory gives the period of oscillation to be $t=4 \pi \epsilon / h_{0}$ in units of the advection time (Johnson, 1984). The linear theory applies when the topographic time is much less than the advection time $\left(\epsilon / h_{0}<<1\right)$. The topographic time is given by the inverse of the magnitude of the vorticity generated over the topography. There is an order of magnitude agreement between the linear theory and the period of rotation of the patch of fluid seen in Figure 4.3. For the our choice of $h_{0} / \epsilon$ the linear oscillation period is about 2.5. We observe that the trapped fluid oscillates around the topography in about 4 advection times, suggesting that non-linearities are of order one importance. In both simulations of Verron and LeProvost (1985) and James (1980), the ejected fluid is often entrained back into the trapped fluid. This process most likely has to do with the finite value of friction in their models, and as such is not seen in our model simulation.

The advection time scale and topographic time scale $\left(\epsilon / h_{0}\right)$ are both order one in the model runs shown here. Over several advection times it is plausible that the system reaches a quasi-steady state with a finite amount of fluid trapped over the topography. Vorticity ejection is probably an important mechanism in the path to the steady state. Since our model is inviscid, we do not reach a steady state in the runs shown. However, over the viscous spin up time in a real fluid, the quasi-steady state would eventually spin up to Ingersoll's (1969) solution discussed in Chapter 2. 
However, the quasi-steady state depends not only on $h_{0} / \epsilon$, but also on how the flow is initiated. We consider this effect next.

To extend both the work of Kozlov (1983) and Verron and LeProvost (1985) we consider the effect of turning on the background flow over different amounts of time. We choose

$$
U=U_{0} \tanh \left(t / \tau_{u}\right) .
$$

In the two examples (Figures 4.2 and 4.3 ) the background flow was turned on abruptly at $t=0$. In the examples discussed below, $\tau_{u}$ is finite. In the case of Figure 4.5 we use the same height of the topography, but turn on the background flow over 4 advection time scales. We calculate the amount of marked fluid that remains over the topography as a function of time (Figure 4.6). It can be seen that, as time increases, the amount of shed fluid increases, while as $\tau_{u}$ increases, the amount of shed fluid decreases.

As discussed in Chapter 2, Johnson (1984) suggested that Ingersoll's (1969) solution may be set up in an initial value problem similar to the one considered here. He assumes that the solution is set up over an advection time. Johnson (1984) calls this solution the maximum retention solution because in it the maximum amount of fluid is retained over the topography. There are intermediate retention solutions that are introduced in Chapter 2. In these solutions, the trapped fluid is contained within a circular contour with center $x=0, y=2 \epsilon / h_{0}$ and radius $r_{0}$ that has magnitude anywhere from zero up to Ingersoll's (1969) solution radius of $r_{0}=r_{c}=1-2 \epsilon / h_{0}$. These states were found for intermediate values of the undetermined constant $C$ in Chapter 2. This provides an infinite set of possible steady solutions where some of the fluid is shed downstream and the rest is trapped over the topography. The difference between these solutions and Ingersoll's (1969) solutions is that there are closed streamlines located outside the circle of radius $r_{0}$. Because of this, they can only exist for times short compared to $\tau$, the spin up time. 

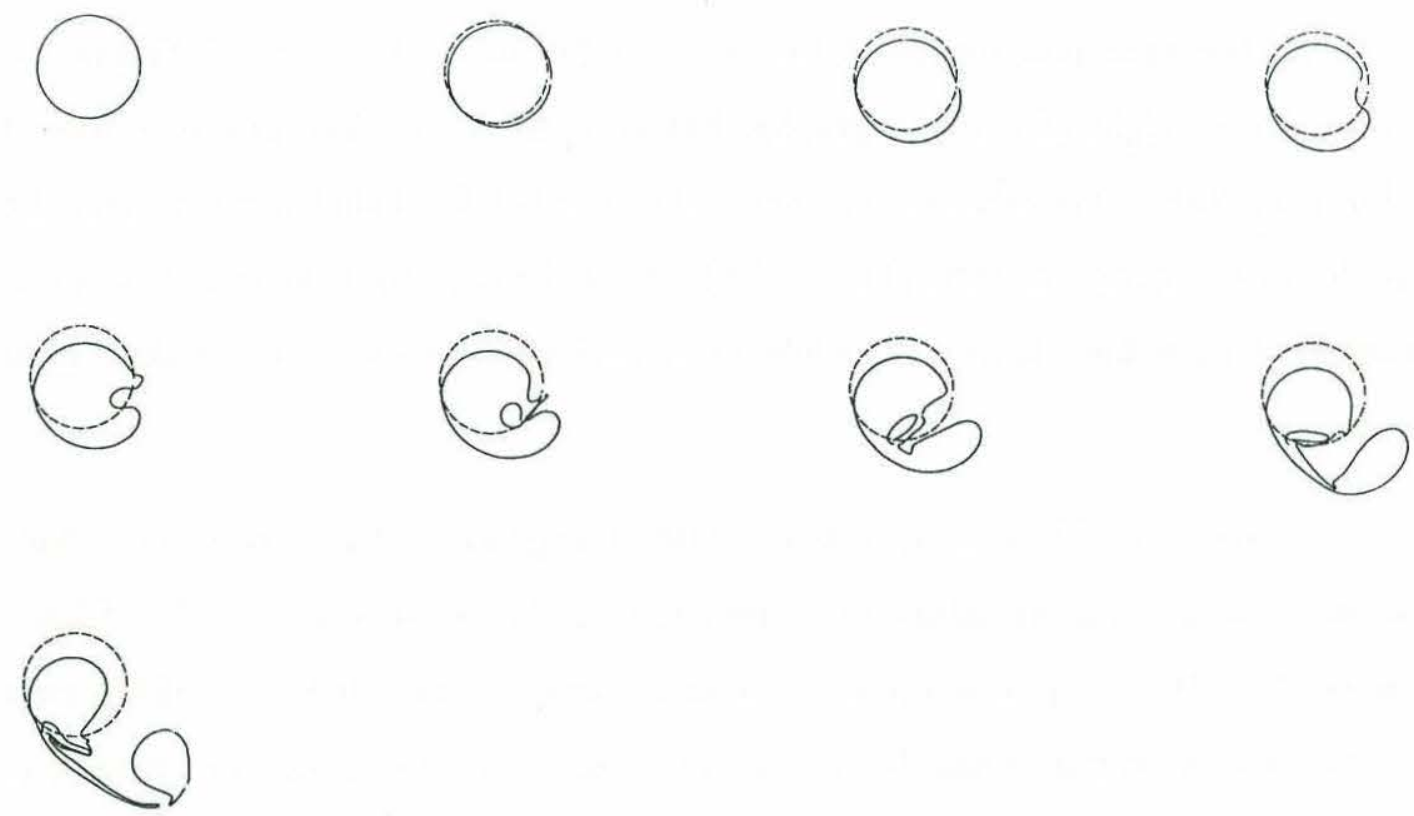

Figure 4.5: Time evolution of the contour for a case when part of the fluid is trapped near the contour. $h_{0} / \epsilon=5$ and the flow is turned on slowly with time scale $\tau_{u}=4$. A snap shot of the contours is taken at $t=0,1,2,3, \ldots 8$. 


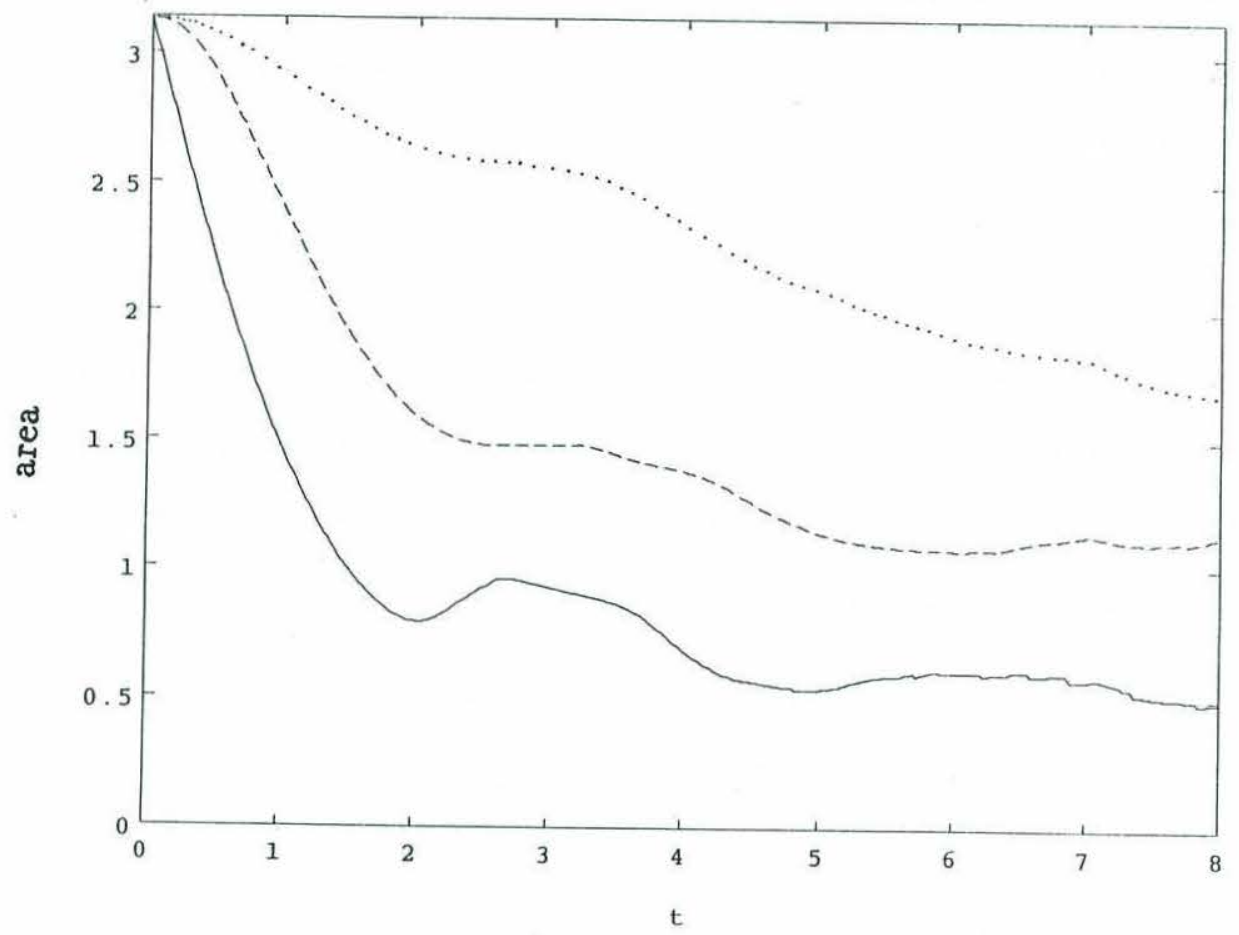

Figure 4.6: The area of the contour that remains over the topography as a function of time when $h_{0} / \epsilon=5$. The three lines are for three different value of $\tau_{u}$ in 4.6 . The solid line is for $\tau_{u}=0$, (the flow is turned on abruptly), the dashed line for $\tau_{u}=1$ and finally the dotted line is for $\tau_{u}=4$. As $\tau_{u}$ increases, it is plausible that approaches 1.13 , the amount of fluid left over the topography in the maximum retention solution. 
The family of solutions are similar to Ingersoll's solution; for $r>1$ they are

$$
\psi=-\frac{h_{0}}{2 \epsilon} \ln r+\frac{K}{2 \pi} \ln \frac{r^{\prime}}{r_{0}}-r \sin \theta-1,
$$

and for $r<1$ they are

$$
\psi=-\frac{h_{0}}{2 \epsilon}\left(r^{2}-1\right)+\frac{K}{2 \pi} \ln \frac{r^{\prime}}{r_{0}}-r \sin \theta-1,
$$

where $K=\pi h_{0} r_{0}^{2} / \epsilon$ and $r^{\prime}$ is the radius referenced to $x=0, y=-2 \epsilon / h_{0}$. The fluid is stagnant within the circle of radius $r_{0}$. The potential vorticity within this circle is $h_{0} / \epsilon$, and this value is consistent with the initial value problem discussed above. We numerically tested the stability of this solution to small perturbations and it is stable. As $\tau_{u}$ increases, the amount of fluid remaining over the topography increases, and it is plausible that it approaches $\pi r_{c}^{2}$ as $\tau_{u}$ becomes large. We surmise that the maximum retention solution can be realized by turning on the background flow very slowly. Intermediate retention solutions probably occur when the flow is turned on over a finite time, and a steady state can be reached with some trapped fluid remaining for a time less than the spin-up time.

We compare the possible steady state solutions of this problem with results from the initial value problem (Figures 4.7 and 4.8). In Figure 4.7, three steady solutions are shown. First, we compare the inertial solution in which all of the fluid which originated over the topography has been swept down stream to Ingersoll's (1969) solution where there is a region of trapped fluid and there are no closed streamlines outside of this fluid. Finally, the intermediate retention solution is constructed choosing $r_{0}$ such that $\pi r_{0}^{2}=1.13$, which was the amount of fluid which was retained over the topography in Figure 4.3. In Figure 4.8 we show the velocity field for the last time step of the runs shown in Figures 4.3 and 4.5. Figure 4.8a compares quite well with Figure $4.7 \mathrm{c}$ as we predicted. Note in particular that the stagnation point in Figure 4.7c is located closer to the topography than in Figure 4.7a, and matches more closely the location of the stagnation point in Figure 4.8a. We note that the amount of fluid 
which has potential vorticity $-h_{0} / \epsilon$ is the same in Figure $4.7 \mathrm{a}$ and $4.8 \mathrm{a}$. In addition, Figure $4.8 \mathrm{~b}$ compares well with Figure $4.7 \mathrm{~b}$. These comparisons give strength to our assumption that the maximum retention solution is realized as $\tau_{u}$ becomes large, while intermediate retention solutions are found as quasi-steady states when $\tau_{u}$ is finite. Over time scales comparable to the spin-up time, we expect that Figure 4.7b would give the final steady-state configuration. The fluid trapped within closed streamlines in the maximum retention solution is thus composed entirely of fluid which originated over the topography. In contrast, in the intermediate retention solution, some of the fluid that is trapped within closed streamlines originated upstream.

\subsection{Flow in one layer over finite topography}

As shown in Section 3.3, flow over finite topography can have a very different character from that of flow over quasi-geostrophic topography. In this section time-dependent flow over finite topography in the form of a right circular cylinder is discussed. We refer again to the schematic in Figure 1.1. Because fluid parcels experience finite depth changes, a modified contour dynamics method is used to find the time-dependent behavior of the system. As in the steady solution, we let the transport normal to the boundary and the tangential velocity along the topography be continuous. These matching conditions are more complicated than those needed in the quasi-geostrophic solutions and require a different Green's function than that given in 4.3. Given the topography and any distribution of vorticity, the solution could be found by determining the appropriate Green's function such that the matching conditions are satisfied and integrating the vorticity over all space. Unfortunately, such a Green's function lacks the appropriate symmetry to be able to use contour dynamics directly, and the velocity cannot be determined simply by doing contour integrals. Instead, the solutions are found by applying the boundary conditions independently at each time step. The simplest application of this method is given in Appendix $\mathrm{C}$ where 


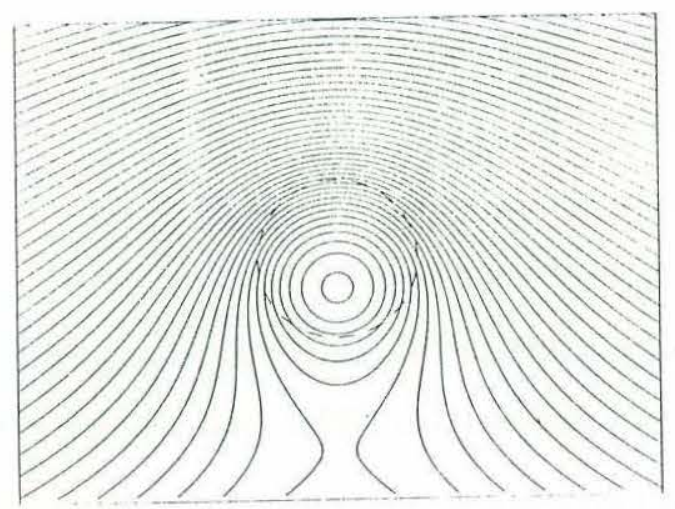

(a)

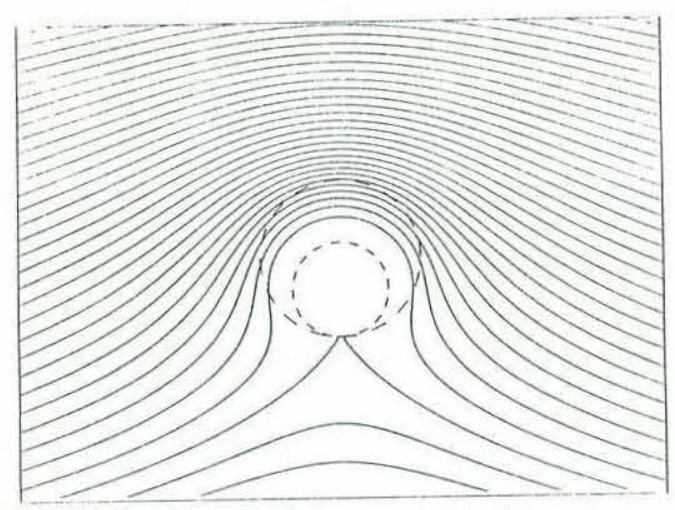

(b)

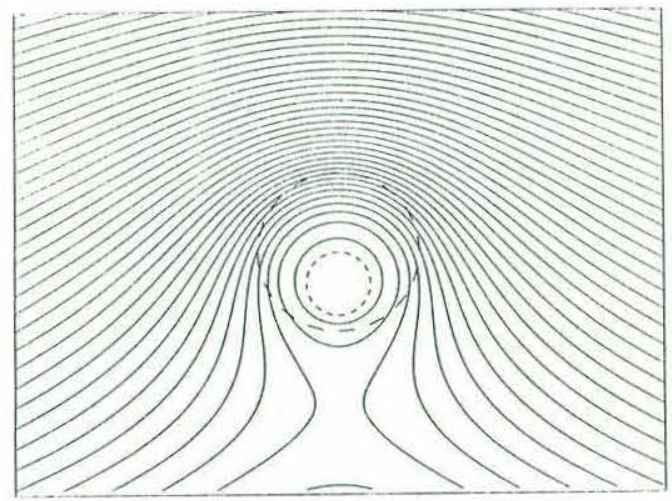

(c)

Figure 4.7: Streamlines for steady inviscid flow over topography when $h_{0} / \epsilon=5$. (a) The inertial solution $\psi^{(a)}$, (b) Ingersoll's (1969) solution, and (c) the intermediate retention solution with $r_{0}=0.4$. 


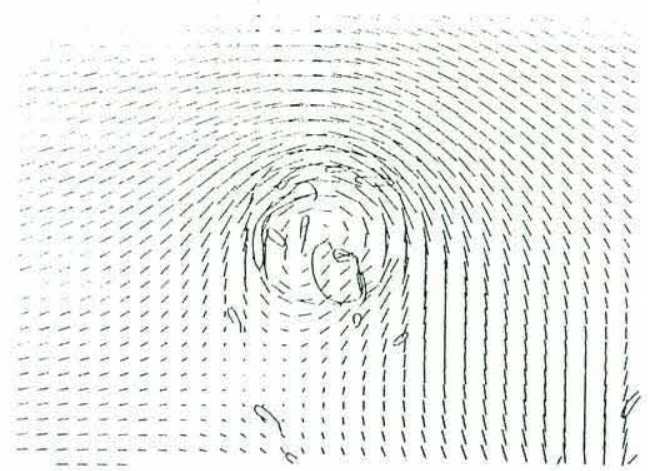

(a)

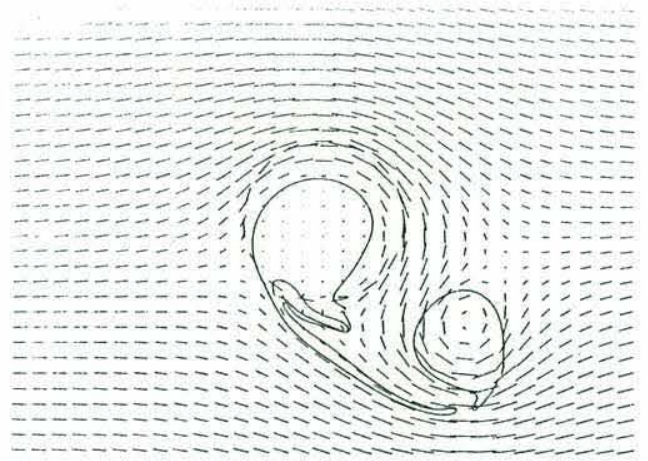

(b)

Figure 4.8: Velocity field in the time dependent model when $h_{0} / \epsilon=5$. (a) $\tau_{u}=0$ and $t=9$, (b) $\tau_{u}=4$ and $t=8$. 
the problem of a finite patch of vorticity impinging on an island in a barotropic fluid is addressed. We find two dynamical regimes that we did not observe in the quasigeostrophic simulations due to the different dynamics in the finite depth model.

\subsubsection{Numerical method}

In this section, a modified contour dynamics method is discussed that allows solution of time dependent evolution of flow over finite topography. Traditional contour dynamics must be altered because the Green's function for the problem lacks the symmetry so that the area integral in 4.2 can not be transformed to a contour integral. If the Green's function is composed of a linear combination of functions $g_{i}$, then each $g_{i}$ must have

$$
\frac{\partial g_{i}\left(\mathbf{x}, b f x^{\prime}\right)}{\partial x}= \pm \frac{\partial g_{i}\left(\mathbf{x}, b f x^{\prime}\right)}{\partial x^{\prime}}
$$

The same condition must hold for the $y$ and $y^{\prime}$ derivatives. Because of the lack of symmetry these condition do not hold for the problem at hand; it is solved instead by applying the boundary conditions explicitly at each time-step. In other words, the problem is divided into a homogeneous solution, driven by the application of the boundary conditions, and a particular solution which is simply the appropriate contour integral using the logarithmic Green's function given in 4.3 which is valid everywhere when there are no matching conditions. The homogeneous solution is the zero potential vorticity solution which must be added so that the matching conditions are satisfied and is composed of a Fourier series.

Unlike the situation in which there is flow over a quasi-geostrophic bump, the induced cyclonic and anticyclonic relative vorticities have different magnitudes when the depth is finite. The scaled potential vorticity equation is given by

$$
\partial_{t} q+\frac{1}{1-h} J(\psi, q)=0
$$


where the potential vorticity $q$ is

$$
q=\frac{1+\epsilon \nabla \cdot \frac{\nabla \psi}{1-h}}{1-h}
$$

where $\epsilon$ is the Rossby number and $h$ is the height of the topography scaled by the total depth of the fluid as before, and $\psi$ is the transport stream function. Both $h$ and $\epsilon$ can be order one. The reader is referred to Section 3.3 and Appendix B for additional comments on the consequences of these equations. Before the background flow has been turned on, the upstream fluid has potential vorticity $q=1$ while the potential vorticity of the fluid over the topography has value $q=1 /\left(1-h_{0}\right)$ where $h_{0}$ is the height of the topography. When the background flow is turned on, the fluid over the topography is pushed off and stretched, gaining cyclonic relative vorticity

$$
\zeta=\frac{h_{0}}{\epsilon\left(1-h_{0}\right)}
$$

while the fluid that has been brought from upstream over the topography has anticyclonic relative vorticity

$$
\zeta=-\frac{h_{0}\left(1-h_{0}\right)}{\epsilon}
$$

In the quasi-geostrophic limit, $h_{0} \rightarrow 0$ but $h_{0} / \epsilon$ finite, we find that 4.9 and 4.10 approach the same limiting magnitude. This introduces an additional complication into the numerical method, because now the potential vorticities in the two regions do not cancel exactly, unlike the cancellation in region B in Figure 4.1 that we took advantage of in Section 4.2.

The problem is solved at each time step by considering the region $r>1$ separately from the region $r<1$. In each region the contour integral is done only over the part of the contour contained within that region using the simple logarithmic Green's function. Then the matching conditions are satisfied. To do this, a solution with zero vorticity is added so that the two regions can be matched. The homogeneous solution chosen has velocity that decays far away from the topography. In particular, 
the homogeneous (zero potential vorticity) solution can be written

$$
\psi^{i}=\sum_{-\infty}^{\infty} a_{n} e^{-i n \theta} r^{|n|}
$$

for $r<1$ and

$$
\psi^{o}=\sum_{-\infty}^{\infty} b_{n} e^{-i n \theta} r^{-|n|}+b \ln r
$$

for $r>1$.

The contribution to the velocity at $r=1$ from the contour integral is then calculated separately for $r=1^{+}$and $r=1^{-}$using the contribution to the contour integral only within the appropriate regions $(r>1$ or $r<1$ respectively), (Figure 4.9). The velocities are calculated at $2^{m}=N$ points evenly spaced in $\theta$ at $r=1$, in cartesian coordinates first, and then are transformed to polar coordinates since the matching conditions are applied to the radial and azimuthal velocities. The transformation is applied via

$$
\begin{gathered}
\hat{r}=\hat{i} \cos \theta+\hat{j} \sin \theta, \\
\hat{\theta}=-\hat{i} \sin \theta+\hat{j} \cos \theta,
\end{gathered}
$$

and

$$
\begin{aligned}
& \hat{i}=\hat{\rho} \cos \theta-\hat{\theta} \sin \theta, \\
& \hat{j}=\hat{\rho} \sin \theta+\hat{\theta} \cos \theta .
\end{aligned}
$$

A Fast Fourier Transform is performed on the polar velocities at $r=1$ to decompose them into modes in $\theta$ such that

$$
u\left(r=1^{-}, \theta\right)=\sum_{n=-N / 2}^{N / 2} u_{n}^{i} e^{-i n \theta}
$$

and

$$
v\left(r=1^{-}, \theta\right)=\sum_{n=-N / 2}^{N / 2} v_{n}^{i} e^{-i n \theta}
$$

where now $u$ is the radial velocity and $v$ is the azimuthal velocity. Likewise, similar equations can be written with subscripts $o$ for the velocity at $r=1^{+}$. 


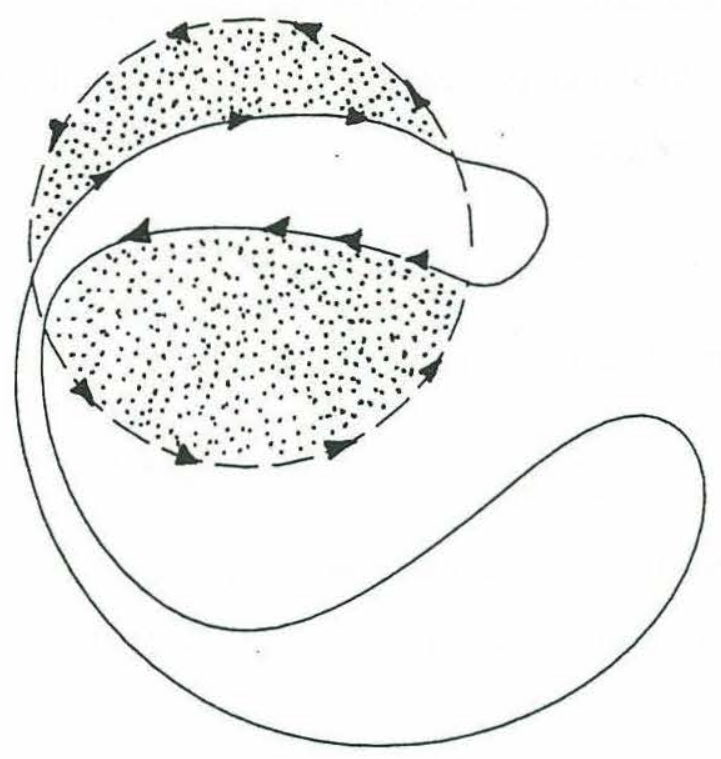

(a)

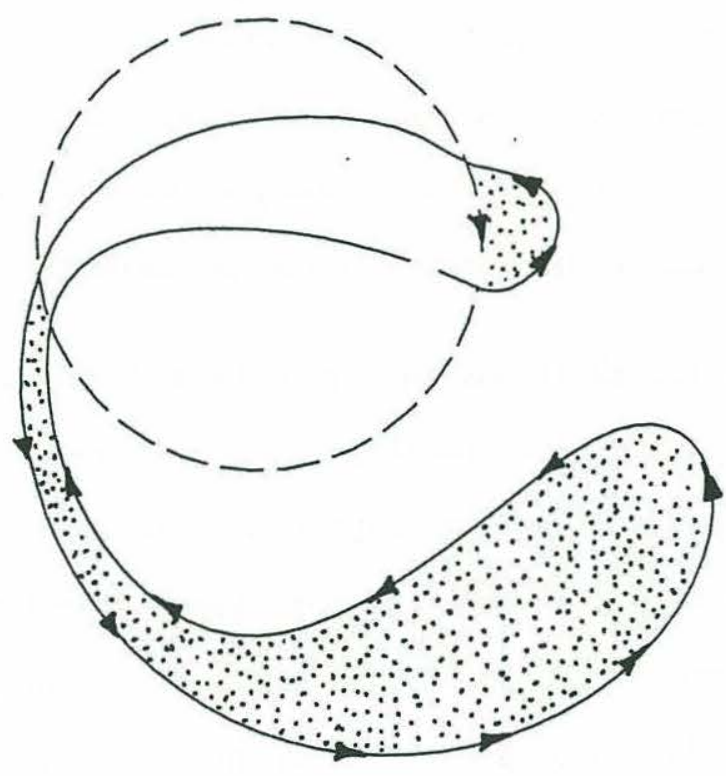

(b)

Figure 4.9: A schematic of the contour integration for the finite topography model. (a) The contour integrals done for points with $r<1$ and (b) for points with $r<1$. The arrows indicate the direction of counter-clockwise integration. The dotted region in (a) has relative vorticity $\zeta=-h_{0}\left(1-h_{0}\right) / \epsilon$ while the dotted region in (b) has relative vorticity $\zeta=h_{0} / \epsilon\left(1-h_{0}\right)$. 
The $a_{n}$ 's, $b_{n}$ 's and $b$ can be found by applying the matching conditions at $r=1$. Remembering that the stream function is the transport stream function, the matching conditions reduce to

$$
i n a_{n}+\left(1-h_{0}\right) u_{n}^{i}=i n b_{n}+u_{n}^{i}
$$

for the transport normal to the edge of the topography to be continuous and

$$
\frac{n a_{n}}{1-h_{0}}+v_{n}^{i}=-n b_{n}+v_{n}^{o}+b \delta_{n, 0}
$$

for the tangential velocity to be continuous. From 4.13 and 4.14 , the coefficients $a_{n}$ and $b_{n}$ in 4.11 and 4.12 can be calculated. We note that for a real solution $a_{n}=a_{-n}^{*}$ and $b_{n}=b_{-n}^{*}$.

To find the velocity field, the contour integral is calculated for the region in which the point of interest lies $(r<1$ or $r>1)$, and then the series solution is summed for the $x$ and $y$ of the point of the contour in question. Typically 16 modes are used in the decomposition. Mass is conserved, as long as the contours are not too broken up as discussed in Section 4.2. Otherwise the mass is conserved within one percent. Vorticity (mass) conservation is no longer equivalent to area conservation.

The contour integrals themselves are more difficult to evaluate than those in traditional contour dynamics because they have to be calculated for the regions $r>1$ and $r<1$ separately. The algorithm used to calculate each line integral is done in several steps as illustrated in Figure 4.9. First, the intersection points of the contour with the edge of the topography, the circle of radius 1 , are found. Whether the contour is entering or exiting the circle, in the sense of the direction of the integration, is also recorded as a vector. Then the intersection points are ordered in $\theta$, sorting the intersection flags in the same way. For the part of the integral on the contour the integration is done until the contour crosses the circle, then a point is interpolated onto the circle which is between the two points on the contour on either side of the circle. The integrals around the arc of the circle must then be performed to complete 
the path, and the flag that tells the direction in which the contour has crossed the circle at each intersection point is used to determine which arcs of the circle belong to the contour integral.

\subsubsection{Numerical solutions}

As shown in Section 3.3, an important difference between the quasi-geostrophic model and the finite depth model is the horizontal divergence of the flow field. Because of this divergence, flow is enhanced over the obstacle and some of the flow is divided to go around the obstacle. No closed streamlines are formed in the steady solution as long as the Rossby number is greater than $1 / 4$ no matter how high the obstacle because of the enhancement of the flow over the topography. An additional difference, which is important for the time-dependent simulations, is that the magnitude of the anticyclonic circulation is less than the magnitude of the induced cyclonic circulation. These differences combine to cause a different evolution of the flow field during the eddy shedding process from that seen in the quasi-geostrophic model.

A series of numerical experiments is examined to show the effects of the finite topography. In order to obtain results here which we can compare directly with the quasi-geostrophic runs we let $h_{0} / \epsilon=5$ in all of the examples that we show for flow over finite topography. In each run, we vary $h_{0}$ only, and the Rossby number changes implicitly since $\epsilon=h_{0} / 5$. In order to study the evolution of the trapped eddy, only runs with $\epsilon<1 / 4$ are considered. The flow over the topography is faster. In both cases, after several advection times, the contour has a $\mathrm{C}$ shape as we saw in Figure 4.3 .

When the height of the bump is one quarter of the water depth (with a Rossby number of .05), the shed eddy rotates more rapidly than in the quasi-geostrophic run in Figure 4.3, (Figure 4.10). This is due to the larger magnitude of the cyclonic 
circulation in the finite depth model. The development of the upper portion of the $\mathrm{C}$ is faster in the finite depth run because of the enhancement of the velocity over the topography. The patch of ejected fluid that originated at the upper part of the $\mathrm{C}$ is trapped more strongly to the shed eddy. The shed eddy is ejected further to the south.

When the height of the topography is even larger, $(h=0.5$ and Rossby number of 0.1 ) the eddy shedding process begins to look different (Figure 4.11). The two extremities of the $\mathrm{C}$ result in two places in which fluid can be shed. The fluid is moved in the clockwise direction due to the anticyclonic vorticity over the topography and is stretched and advected across the northern half of the bump as before. Eventually, some of this fluid in the upper part of the $\mathrm{C}$ is pushed off the bump and escapes. The progression of the fluid over the northern part of the topography is faster because the background flow is enhanced over the topography. In the quasi-geostrophic run, this patch of fluid is also advected southward by the anticyclonic circulation and is then stretched and caught up by the southern end of the $\mathrm{C}$, but here the northern part of the $\mathrm{C}$ is shed before it comes into contact with the southern eddy. There are therefore potentially two cyclonic eddies created. The northern eddy is swept into the part of the flow that is split by the topography and escapes downstream. The velocity in Figure 4.12 shows that both the southern and the northern cyclonic eddies have closed circulation patterns and are composed of fluid which originated over the topography.

When the topography takes up most of the water column $(h=0.75$ and Rossby number of 0.15 ) the behavior changes yet again (Figure 4.13). In this regime, there is oscillatory behavior, and the C-shaped structure is no longer seen. Instead, the fluid rotates around the topography and oscillates on and off the topography. The period of the oscillation is close to 2 advection times, reasonably close to the linear theory value of $2.5\left(4 \pi / \epsilon h_{0}\right)$. The mass of the fluid is conserved, but not the area of the 

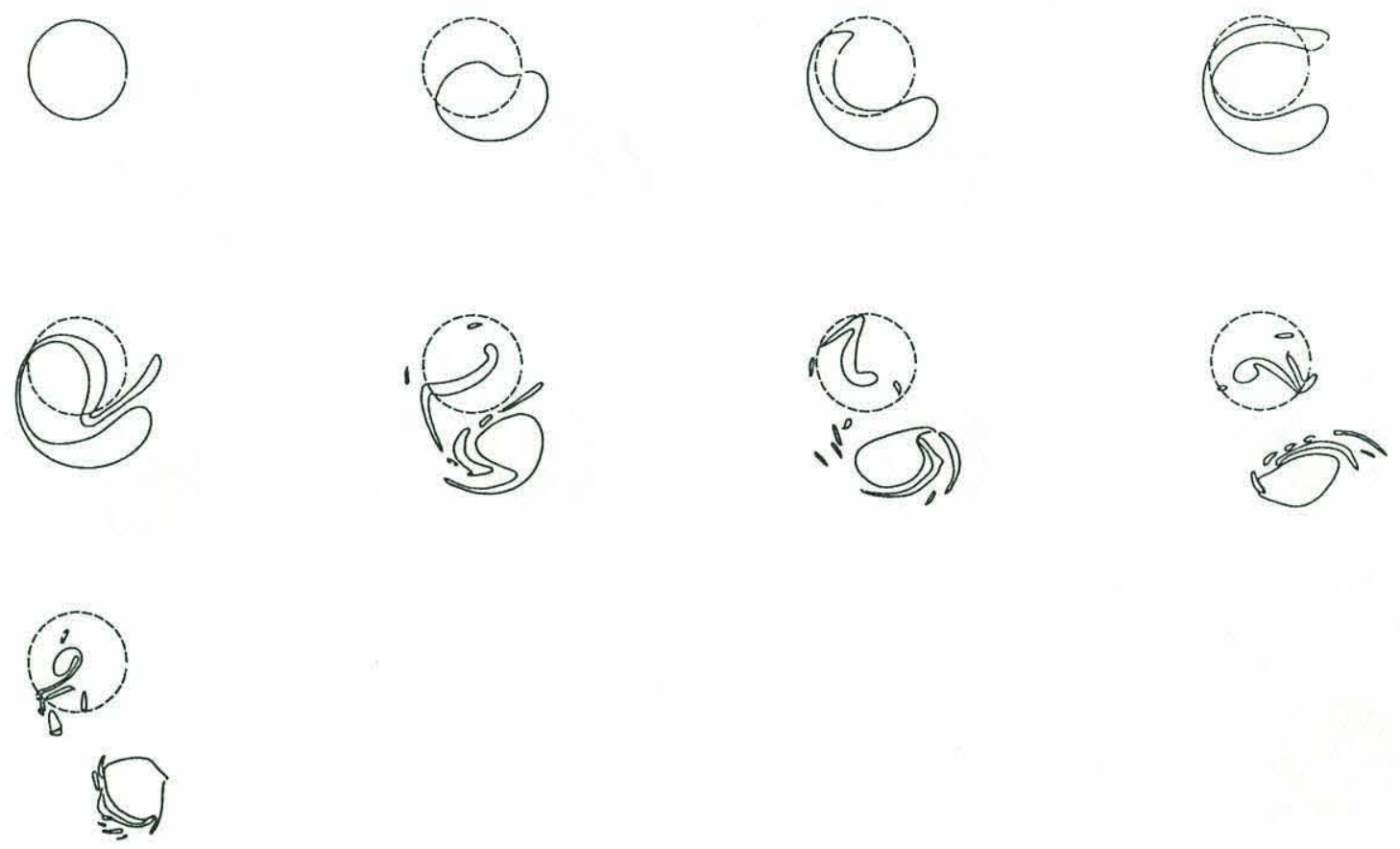

Figure 4.10: Time evolution of the contour for the finite depth model when $h_{0}=0.25$ and $h_{0} / \epsilon=5$ as in Figure 4.3 for $t=1,2, \ldots 8$. 

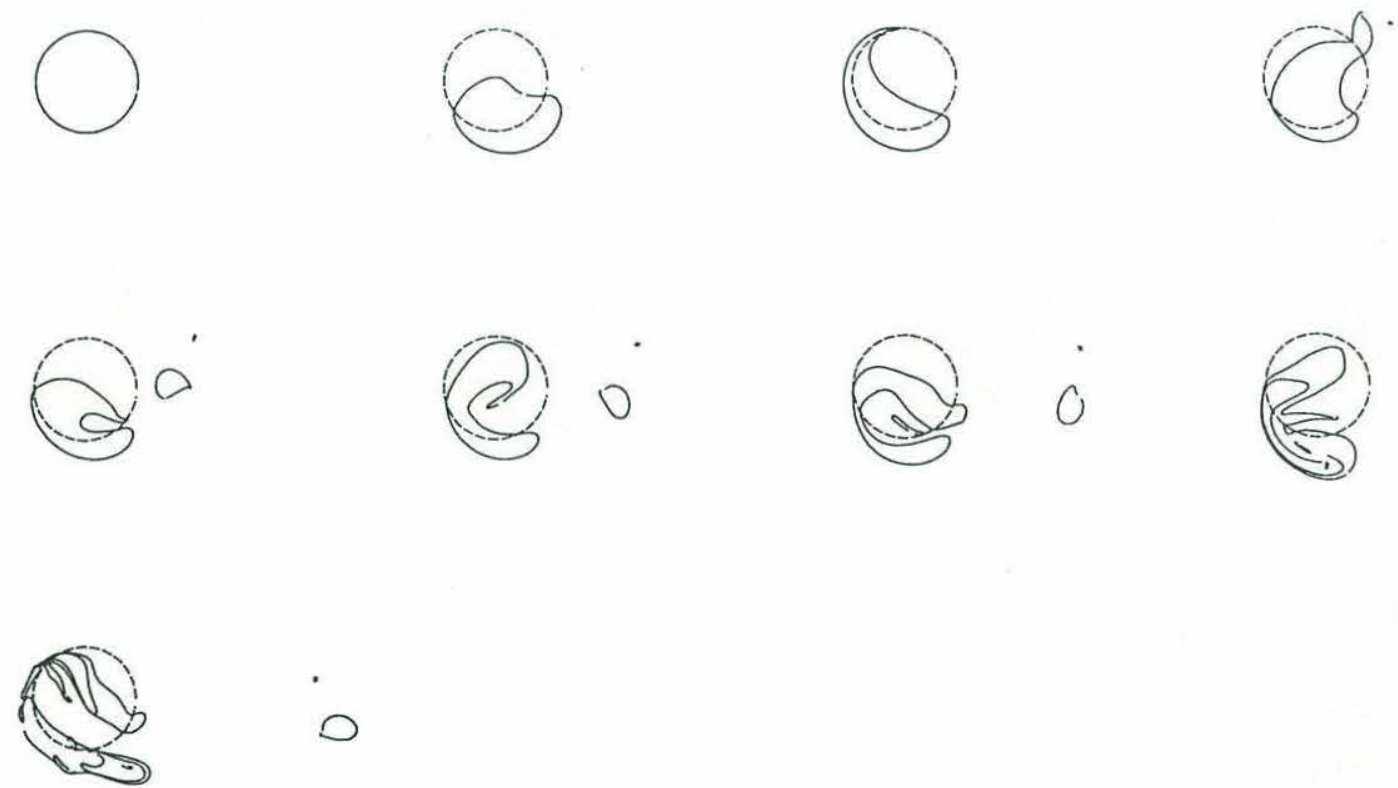

$\bigcirc$

Figure 4.11: Time evolution of the contour for the finite depth model when $h_{0}=0.5$ at $t=1,2, \ldots 8$. 


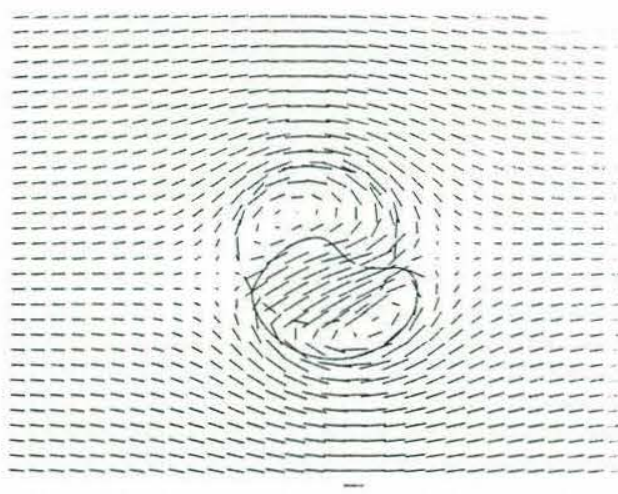

(a)

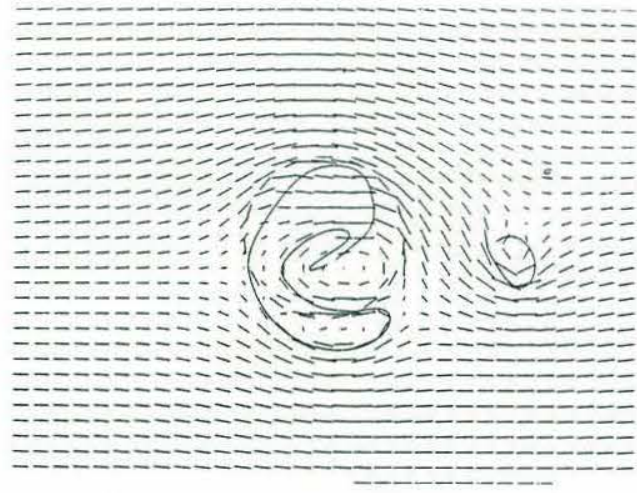

(c)

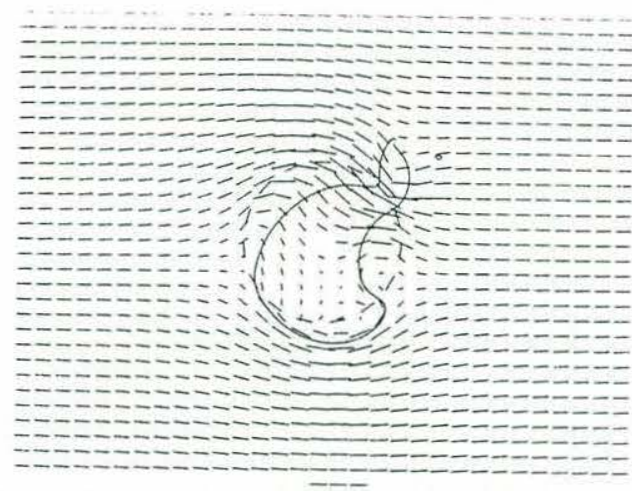

(b)

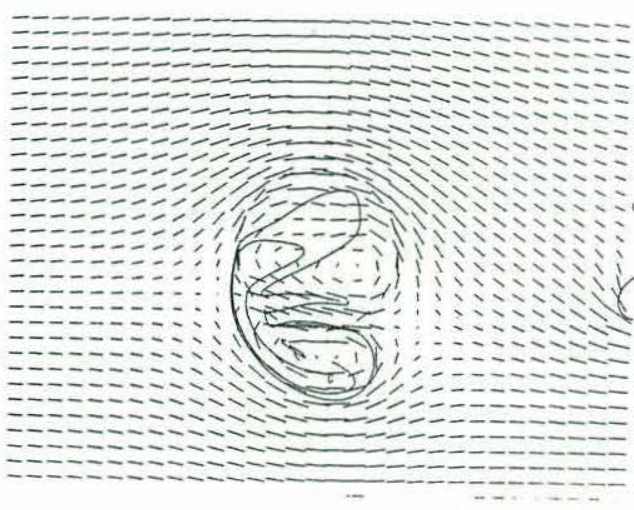

(d)

Figure 4.12: Flow field for the case shown in Figure 4.11. (a) $t=1$, (b) $t=3$, (c) $t=5$, and (d) $t=7$. 
contour, as can be seen easily in this example. Initially, the fluid is pushed off of the topography, generating anticyclonic circulation over the topography, and rotating the fluid around the topography in a clockwise direction as a dipole. Then, the upstream flow pushes the fluid back over the topography when the fluid has moved to the west side of the topography. Then the process begins over again. The velocity is shown in Figure 4.14 for the first oscillation period. It can be seen that the flow field induced is nearly symmetric about the $x$ axis. The oscillation is associated with a dipole field that moves around the topography. The flow is enhanced over the topography as required in the finite depth model. As can be seen in Figure 4.13, the oscillation is fairly stable, the wiggles that appear on the contour could be a numerical instability, and not a physical one.

Because this model involves finite depth changes, while not allowing vertical variations in the horizontal velocity, there are several potential problems that we hope do not affect the qualitative description of the eddy shedding process. First, because the depth changes a finite amount over an infinitesimal distance, one would expect large vertical velocities at the boundary of the topography, which results in a breakdown in one of the physical approximations of the model, that the vertical variations of the horizontal velocity are negligible. This problem is considered in Section 3.3 and Appendix B. The second potential problem is numerical. Consider two points on the contour that straddle an intersection of the contour and the circle with radius 1 , the edge of the topography. The point just inside the topography has velocity $U$ while the point just outside the velocity has velocity $U\left(1-h_{0}\right)$ due to the conservation of radial transport. Thus, over the next time step, the point over the topography is moved a distance $U \Delta t$ while the point not over the topography is moved $U \Delta t\left(1-h_{0}\right)$. The point originally over the topography can overtake the point not over the topography. This error can create kinks in the contour, possibly those seen in Figure 4.13. The errors associated with this are of the size of the time step $\Delta t$, while the error in the time stepping scheme is order $\Delta t^{2}$, so the results are less 

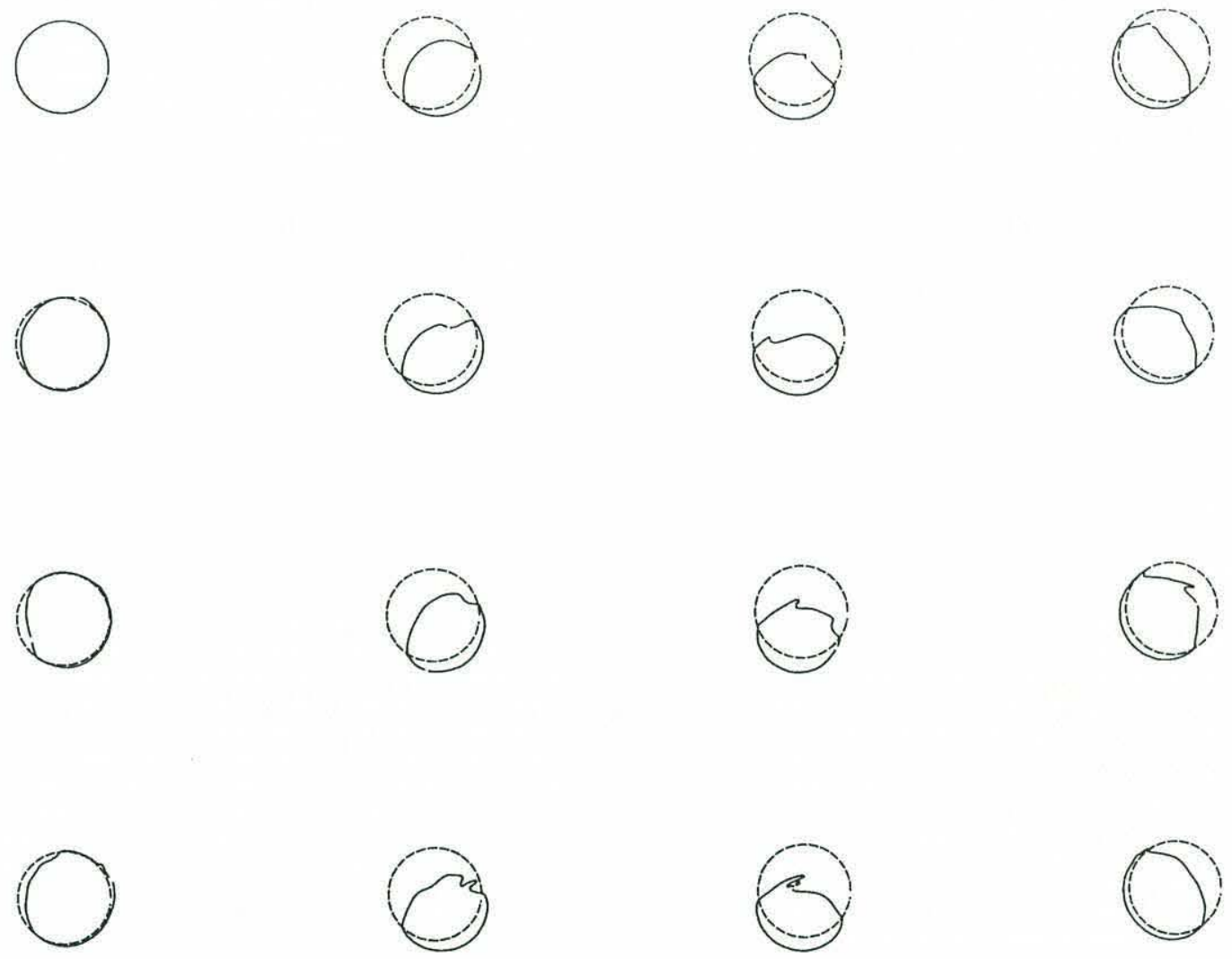

Figure 4.13: Time evolution of the contour for the finite depth model when $h_{0}=0.75$ and $h_{0} / \epsilon=5$ as in Figure 4.3 for $t=0.5,1,1.5, \ldots, 7.5$. 


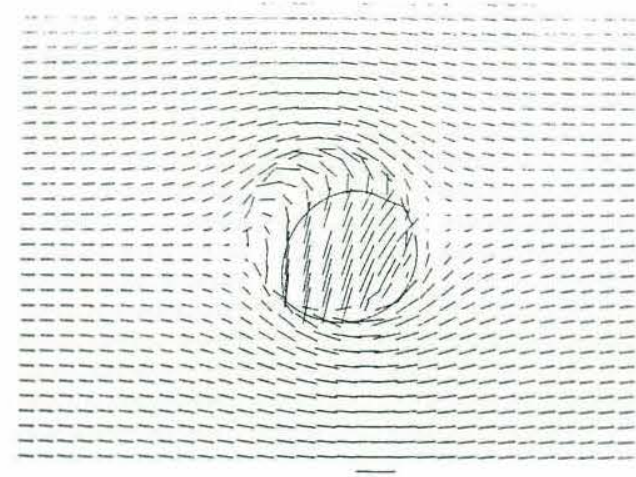

(a)

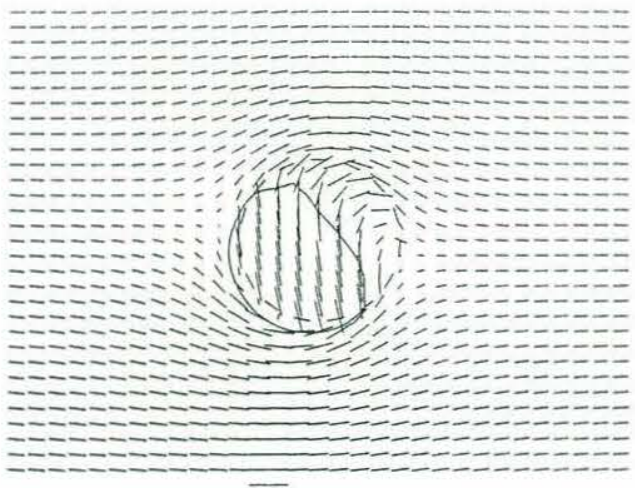

(c)

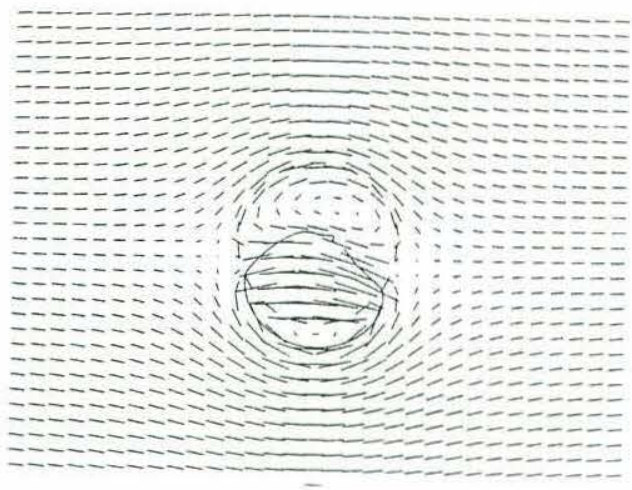

(b)

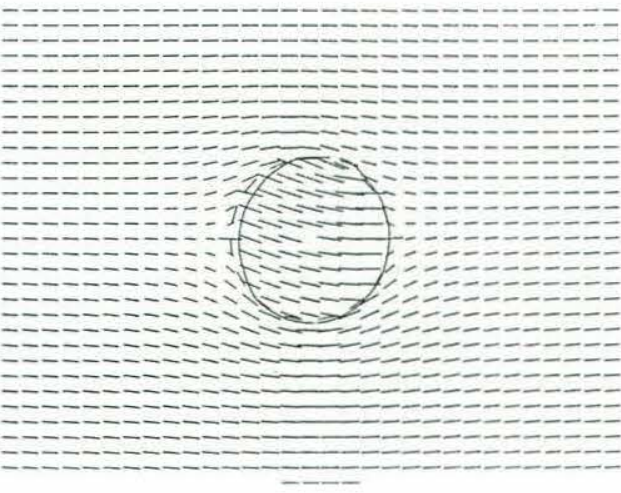

(d)

Figure 4.14: Flow field for the case shown in Figure 4.13. (a) $t=0.5$, (b) $t=1$, (c) $t=1.5$, and (d) $t=2$. Note that the arrows indicate velocity not transport, and the radial velocity is discontinuous at $r=1$. 
accurate because of this. In order to improve the numerical stability of the method, one must then reduce the time step. This was done for a case which was initially numerically unstable in which the vorticity does not remain conserved. When the time step was reduced, the solution became numerically stable and the total amount of vorticity was conserved.

The model illuminates some of the effects of flow over finite topography and how the eddy shedding process differs from the quasi-geostrophic process. As the topographic height is increased for a fixed $h_{0} / \epsilon$ ratio, the model goes through a progression of different behaviors. When the topography is small, the model behaves like the quasi-geostrophic model; the contour that marks the fluid that originates over the topography is deformed into a C-shape, and then the northern end of the $\mathrm{C}$ is advected to the south where it is sheared by the southern end and these two pieces become the shed eddy. When the height of the topography is increased further $\left(h_{0}=0.5\right)$, the northern piece and southern piece are shed independently as the northern piece is advected by the enhanced flow over the topography, and two cyclonic eddies are created. Finally, when the topography is quite large, an oscillatory regime is reached where the fluid starting over the topography oscillates on and off the topography and moves clockwise around the topography.

\subsection{Flow in two layers over small topography}

Once we understand the behavior of flow over topography in one layer, the next thing to consider is flow in two layers. The two-layer model is the simplest model that captures the effects of stratification. The equivalent barotropic model is inappropriate for flow over oceanic topographic features, because in the equivalent barotropic approximation, the deeper layer is assumed to be stagnant, and in the ocean, the lower layer where the flow is interacting with topography is much deeper than the 
upper layer but cannot be assumed to be stagnant. In this section, we study the flow over a bump which has fractional height of the same order as the Rossby number. The dynamics of the model are illustrated by considering two situations, one in which the stratification is moderately strong, and one in which the stratification is weak. We choose the topographic height so that comparisons can be made to the quasi-geostrophic runs made in Section 4.2. As in the previous Section 4.2 and 4.3, we concentrate on the situation in which there is partial trapping of fluid over the topography.

The model geometry is identical to the geometry used by McCartney (1975) and is shown in Figure 1.1. Because we are using the method of contour dynamics, we once again restrict ourselves to background flow which is uniform in both the horizontal and the vertical. This restriction reduces the number of free parameters to $h_{0} / \epsilon$ where $h_{0}$ is the height of the topography relative to the total layer depth, $\delta=(1-d) / d$ and $\gamma=L / L_{R}$ where $L$ is the radius of the topography, and

$$
L_{R}^{2}=\frac{g \Delta \rho\left(H-d^{\prime}\right)}{\rho f^{2}},
$$

where $\rho$ is the density of the fluid and $\Delta \rho$ is the density difference between the two layers.

\subsubsection{Numerical Method}

For flow over small topography, the method of solution is the same as for the flow over small topography in one layer, except that the two layer Green's function is used. In particular, the two-layer quasi-geostrophic potential vorticity equations can be written

$$
\partial_{t} q_{n}+J\left(\psi_{n}, q_{n}\right)=0
$$

for $n=1,2$ where

$$
q_{1}=\nabla^{2} \psi_{1}+\gamma^{2}\left(\psi_{2}-\psi_{1}\right),
$$


and

$$
q_{2}=\nabla^{2} \psi_{2}+\delta \gamma^{2}\left(\psi_{1}-\psi_{2}\right)+\frac{h(1+\delta)}{\epsilon} .
$$

The flow field can be found with the method developed in Section 4.2, by solving for the relative vorticity and doing contour integrals to find the velocity. Once again, the magnitude of the induced anticyclonic and cyclonic relative vorticity cancel exactly. The flow can be found by integrating over the contour in the lower layer which delineates the fluid that originated over the topography. The contour over the topography can be done analytically as before. The contribution from the topographic contour integral is given by

$$
\psi_{1}^{t}= \begin{cases}\frac{h_{0}}{\epsilon}\left(\frac{K_{1}(\Gamma)}{\Gamma} I_{0}(\Gamma r)-\frac{1}{4} r^{2}\right) & r<1 \\ \frac{h_{0}}{\epsilon}\left(-\frac{I_{1}(\Gamma)}{\Gamma} K_{0}(\Gamma r)-\ln r\right) & r>1,\end{cases}
$$

and

$$
\psi_{2}= \begin{cases}\frac{h_{0}}{\epsilon}\left(-\delta \frac{K_{1}(\Gamma)}{\Gamma} I_{0}(\Gamma r)-\frac{1}{4} r^{2}\right) & r<1 \\ \frac{h_{0}}{\epsilon}\left(\delta \frac{I_{1}(\Gamma)}{\Gamma} K_{0}(\Gamma r)-\ln r\right) & r>1 .\end{cases}
$$

This is just McCartney's steady solution for flow over small topography when the background shear is zero and corresponds to Merkine and Kalnay-Rivas (1976) bound vortex solution. It is the solution that would be found if all of the fluid that originated over the topography were swept off, and then the background flow were turned off.

The velocities induced by this stream function are found by taking the appropriate derivative in $x$ or $y$. The contribution to the velocities from the contour integral over the contour that moves is given by

$$
\psi_{i}(x, y)=\frac{1}{2 \pi} \iint \frac{h_{0}(1+\delta)}{\epsilon \delta} G_{i j}(R) d x^{\prime} d y^{\prime}
$$

where $R=\left[\left(x-x^{\prime}\right)^{2}+\left(y-y^{\prime}\right)^{2}\right]^{1 / 2}$ and $G_{i j}$ is the Green's function for the effect in layer $i$ of a point vortex in layer $j$ (Polvani, 1988). For this problem, a subset of the $G_{i j}$ 's is needed since the vorticity is zero everywhere except within the region of fluid 
that originated over the topography in the lower layer. These Green's functions are given by

$$
\begin{aligned}
& G_{12}=\frac{1}{1+\delta} \ln R+\frac{1}{1+\delta} K_{0}(\Gamma R) \\
& G_{22}=\frac{1}{1+\delta} \ln R-\frac{\delta}{1+\delta} K_{0}(\Gamma R)
\end{aligned}
$$

where

$$
\Gamma=\gamma(1+\delta)^{1 / 2}
$$

and $K_{0}$ is the modified Bessel function of order zero. The fundamental length scale of the problem is the Rossby radius of deformation, $1 / \Gamma$ in units of the radius of the topography. The velocity is found as before by taking derivatives and converting the area integral into a contour integral.

The interface height can be calculated for any time once the position of the contours are known. The upper layer potential vorticity is always zero; therefore, the interface height can be calculated by finding the relative vorticity in the upper layer:

$$
\eta=\frac{\Gamma^{2} \delta\left(\psi_{2}-\psi_{1}\right)}{(1+\delta)^{2}}=-\frac{\delta \nabla^{2} \psi_{1}}{1+\delta}
$$

from 4.15. The relative vorticity can be calculated directly from the contour integral 4.17:

$$
\nabla^{2} \psi_{2}=v_{x}-u_{y}=\oint_{\partial D} \frac{G_{21}(R)}{R}\left[\left(x-x^{\prime}\right) d y^{\prime}-\left(y-y^{\prime}\right) d x^{\prime}\right] .
$$

It is important to note that there is no singularity in this integral since the point where the vorticity is being calculated is in the upper layer, while the singularities are all in the lower layer.

\subsubsection{Numerical solutions}

In the numerical solutions we let the layer depths be equal and consider two values of stratification. In each run, a contour delineating the fluid that originates over the 
topography in the upper layer is followed throughout the evolution of the flow. This contour is dynamically inactive, but shows where particles that originated over the topography in the upper layer end up. Comparing this contour and the contour that marks the fluid that originated over the topography in the lower layer serves to show how much the two layers are coupled. The results are compared with the one-layer quasi-geostrophic solutions discussed in Section 4.2.

When $\Gamma=0.25$ and $h_{0} / \epsilon=2.5$, the upper layer contour is not coincident with the lower layer contour, and the response is baroclinic (Figure 4.14). When the stratification is strong ( $\Gamma$ small), the layers are uncoupled: the lower layer responds like a one layer fluid such as was described in Section 4.2 with half the depth. Therefore, this value of topographic height can be compared with topographic heights $h_{0} / \epsilon=5$ in the one-layer model. At the same time, the lower layer contour in Figure 4.15 is nearly identical to the contour in Figure 4.3. The upper layer contour moves off downstream with little distortion. The steady solution of this problem for these values of parameters which predicts that some fluid would be trapped over the topography is illustrated by considering the critical height above which closed streamlines form in the steady solution as shown in Figure 3.20. Figure 4.3 shows that some fluid remains over the topography in the initial value problem.

When the stratification is weak ( $\Gamma$ large), the fluid responds more barotropically than in the previous example (Figure 4.16). The contours in the upper and lower layers nearly overlay each other. The fluid responds nearly as a one-layer fluid. Less fluid remains over the topography when the stratification decreases because the fluid is less bottom trapped. This result is predicted from the steady solutions as seen in Figure 3.20. Calculation of the flow field indicates that the flow is nearly barotropic both over the topography and in the shed eddy that is downstream of the topography, although the anticyclonic circulation is slightly larger in the lower layer (Figure 4.17). 

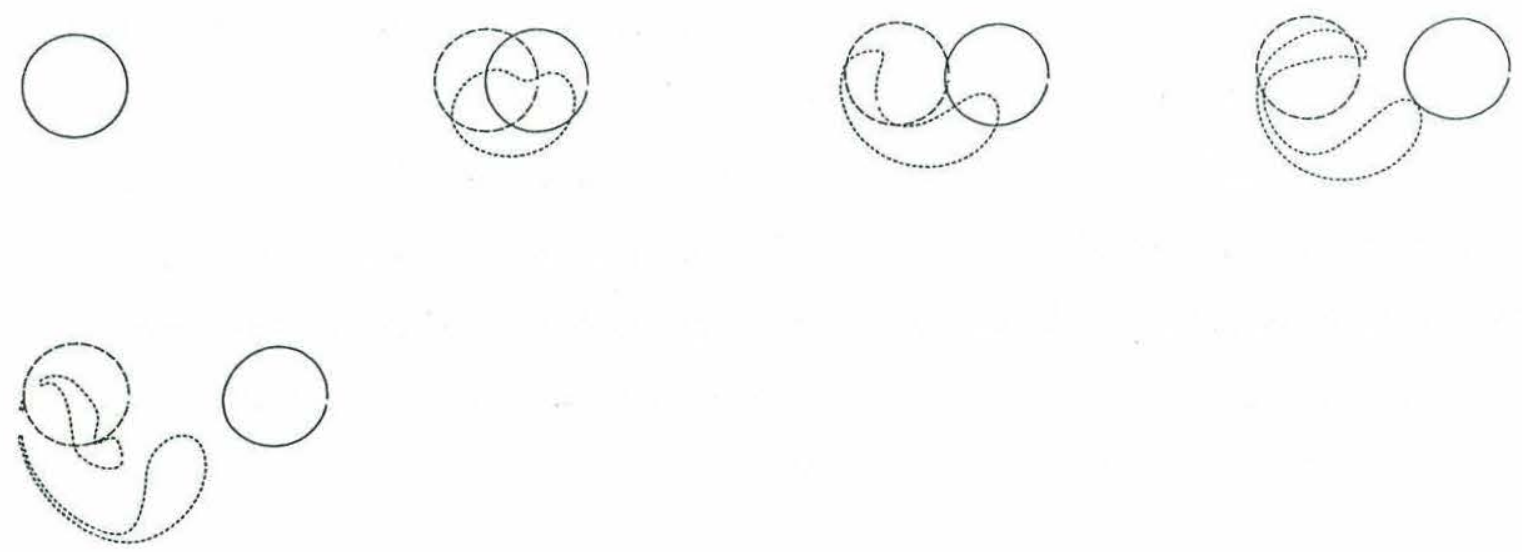

Figure 4.15: Time evolution of the contours which delineate fluid that originated over the topography in the upper layer (solid) and in the lower layer (dashed) in the two-layer small topography model. $\Gamma=0.25, \delta=1$ and $h_{0} / \epsilon=2.5, t=0,1,2,3,4$. The stratification is strong so the two layers are relatively uncoupled. The lower layer contour has nearly the same evolution as the contour in Figure 4.3. The upper layer contour is deformed very little and the fluid escapes downstream. The upper layer contour is simply a passive tracer of the flow in the upper layer. 

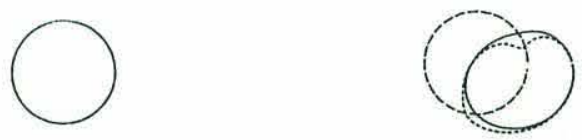

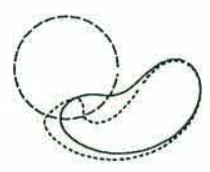

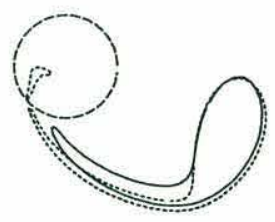

Figure 4.16: Time evolution of the contours that delineate fluid that originated over the topography in the upper layer (solid) and in the lower layer (dashed) in the twolayer small topography model. $\Gamma=4, \delta=1$ and $h_{0} / \epsilon=2.5, t=0,1,2,3,4$. The stratification is weak so the two layers are strongly coupled. Little fluid remains over the topography.

175 


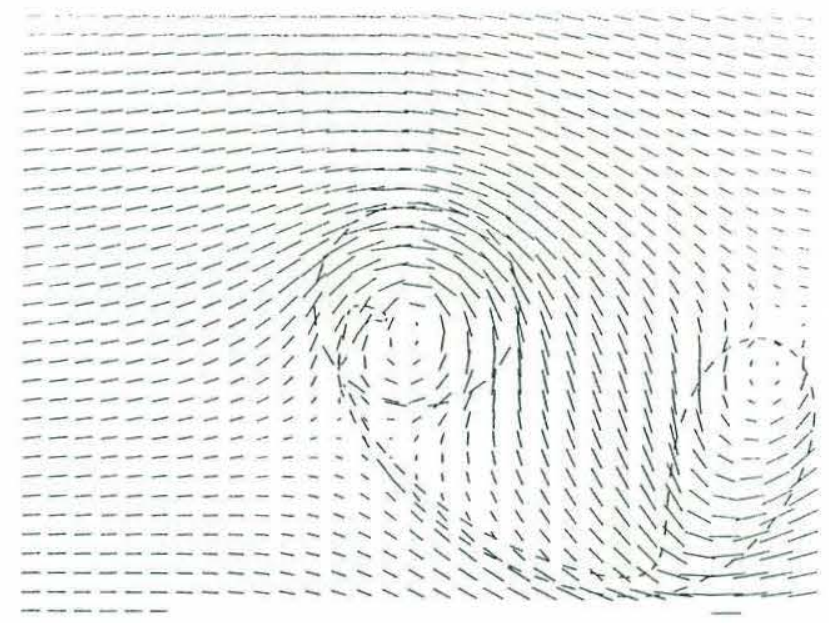

(a)

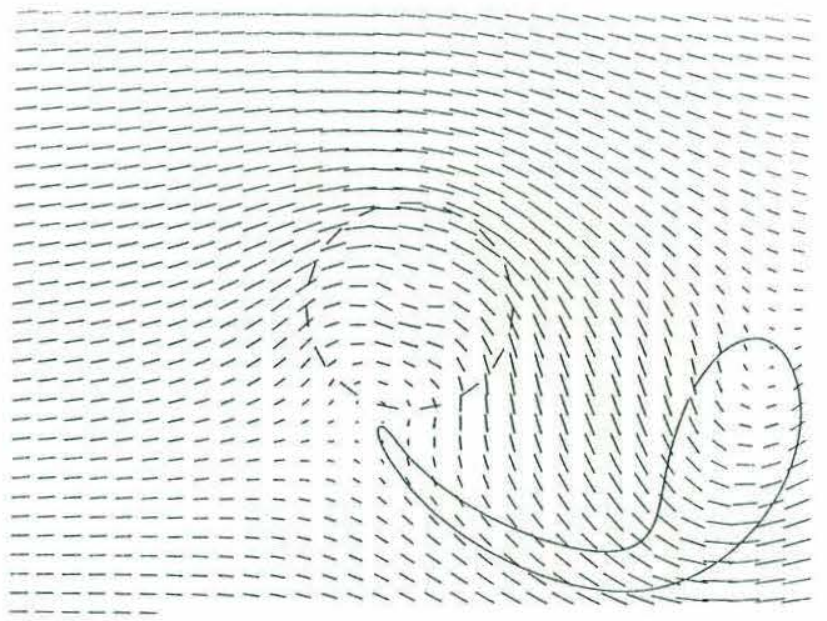

(b)

Figure 4.17: The flow field of the fluid for the case shown in Figure 4.16 at $t=4$. (a) Lower layer, (b) upper layer. The flow in the two layers is nearly identical. The shed eddy has cyclonic circulation, while the flow over the topography is anticyclonic. The response is slightly larger in the lower layer and is bottom trapped. 
The interface is raised over the topography as has been seen in other quasigeostrophic stratified models, and the shed eddy carries a warm anomaly downstream (Figures 4.18 and 4.19). The sign of the interface displacement is the same as is seen in a primitive equation models, (Huppert and Bryan, 1976).

When the topographic height is twice the size as in the case above $\left(h_{0} / \epsilon=5\right.$ and Figure 4.20), the development of the lower layer contour is quite similar to what is seen in Figure 4.3. However, for this case, the upper layer contour no longer overlies the lower layer contour. Even for this small value of stratification, fluid only remains over the topography in the lower layer.

Although as we showed earlier in Section 4.2 the amount of fluid trapped depends on how the flow is initiated, the steady solution provides a way to understand when fluid will be trapped over the topography in the initial value problem. The amount of fluid that remains over the topography is less than predicted from the inertial theory discussed in Chapter 3. Additional fluid which originated upstream is also trapped within closed streamlines over the topography (Figure 4.17). We chose to illustrate how the model depends on the stratification. The flow development can be understood by considering that the two-layer problem behaves fairly similarly to the one-layer example, but with an adjusted topographic height to account for the vertical scale of the bottom trapping. Basic quasi-geostrophic dynamics are illustrated well with these examples.

\subsection{Flow over finite topography in two layers}

The next step in understanding flow over topography in a baroclinic ocean is to consider time-dependent flow over finite topography. The model we use is the same one that is considered in Section 3.3 and is shown schematically in Figure 1.1. The model is of flow impinging on topography which goes all the way through the lower layer, 


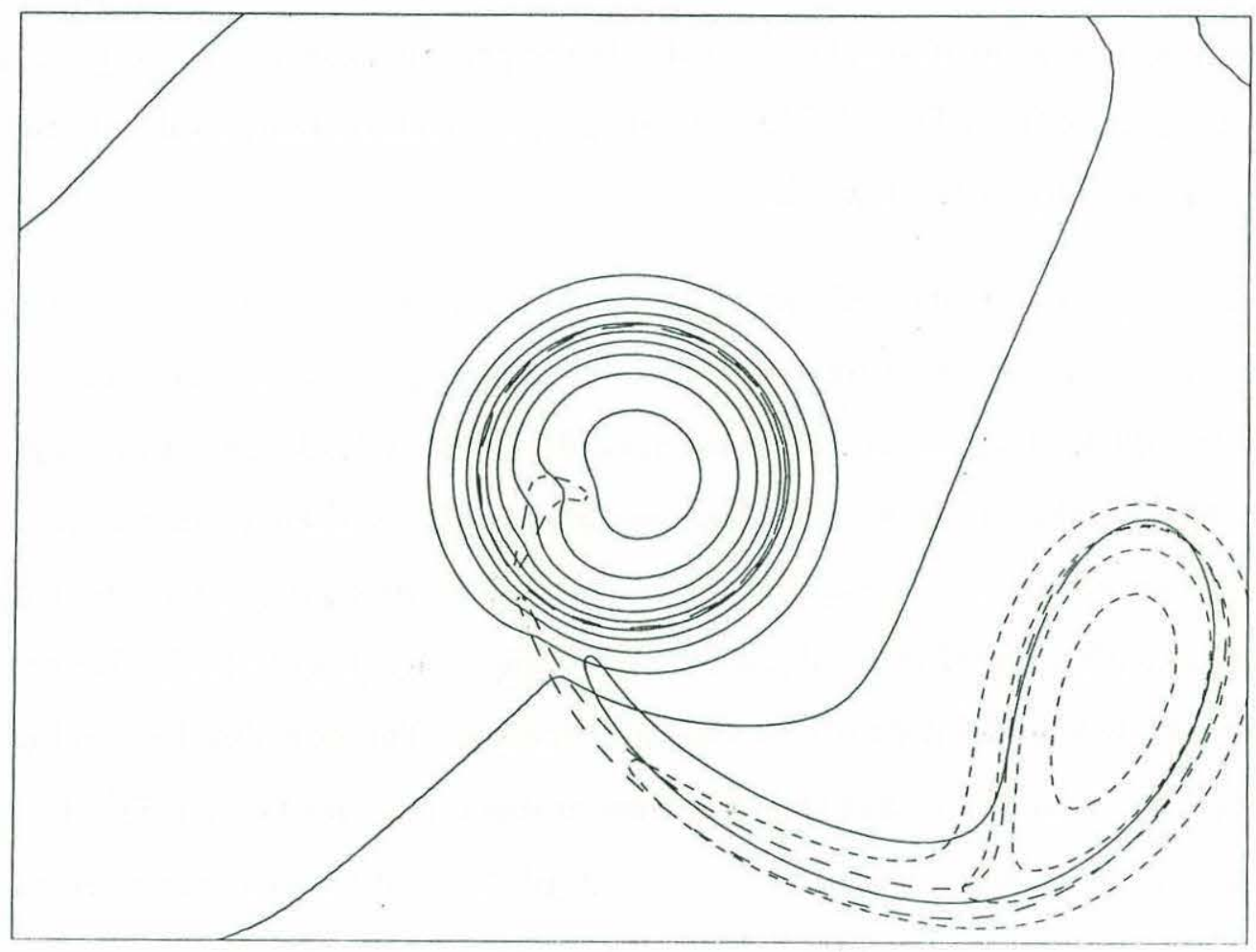

Figure 4.18: The interface displacement for the case shown in Figure 4.16 at $t=4$. Dashed contours indicate negative interface displacement. The interface is high over the topography and is low over the shed eddy. The shed eddy is warm core, cyclonic and bottom trapped. The solid line outside of $r=1$ is the contour which delineates fluid which originated over the topography in the upper layer; the line with the larger dashes delineated the fluid which originated over the topography in the upper layer. 


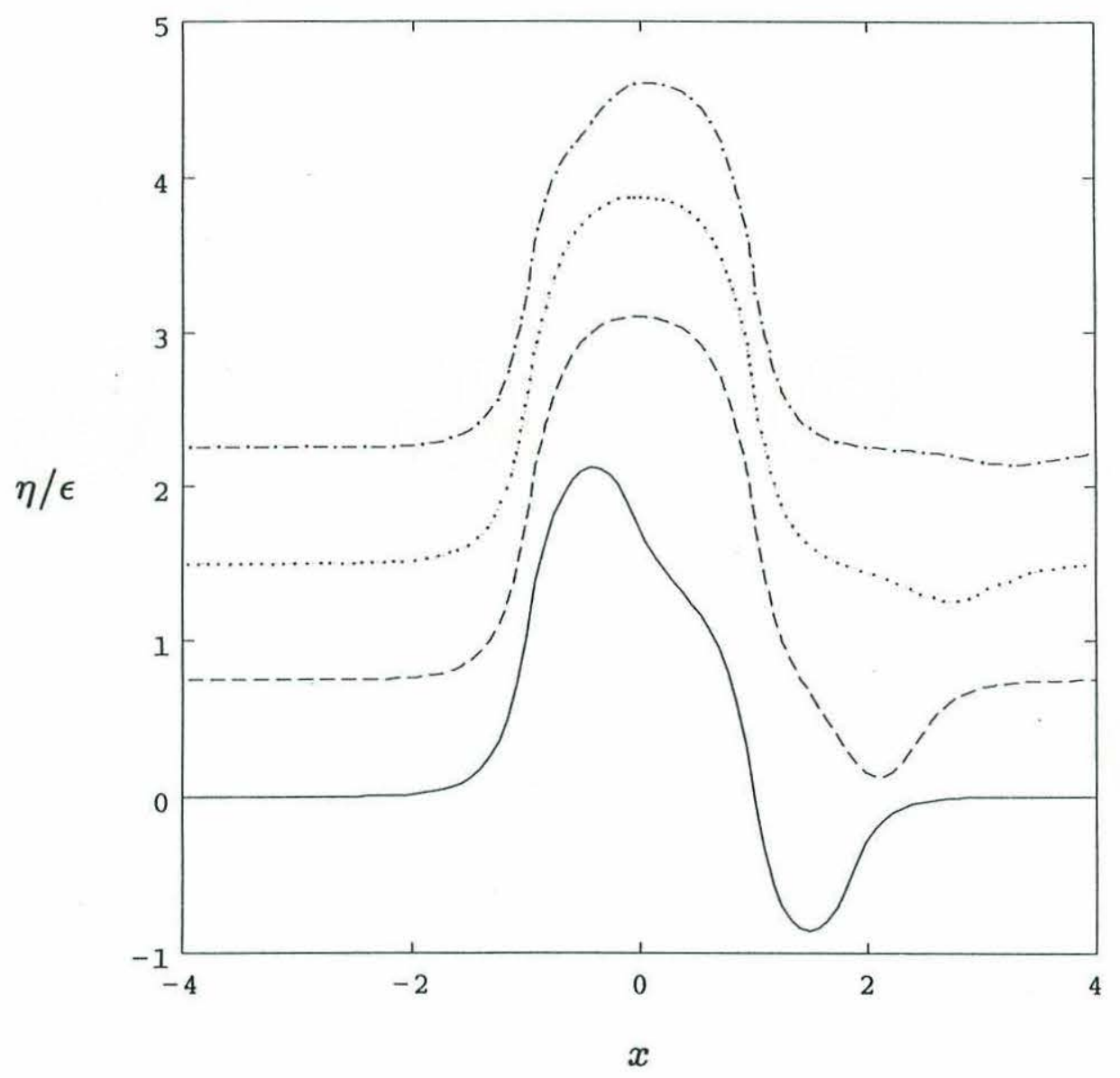

Figure 4.19: The interface displacement for the case shown in Figure 4.16 at a cut along the $x$ axis at $t=1,2,3,4$. The interface is raised near the topography and is lowered over the shed eddy. The shed eddy is warm core and bottom trapped. Notice that the cut is not taken through the center of the eddy, so that the maximum interface displacement due to the shed eddy is larger than shown. The interface is offset by 0.75 for $t=2,1.5$ for $t=3$, and 2.25 for $t=4$. 

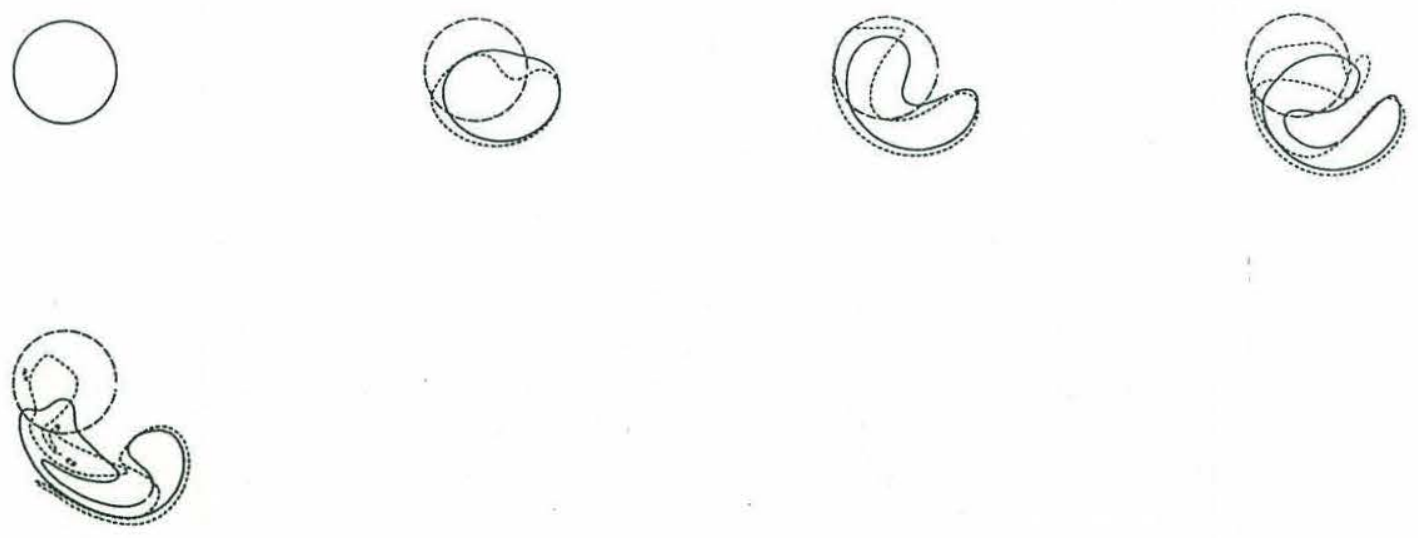

Figure 4.20: Time evolution of the contours that delineate fluid that originated over the topography in the upper layer (solid) and in the lower layer (dashed) in the twolayer small topography model. $\Gamma=4, \delta=1$ and $h_{0} / \epsilon=5, t=0,1,2,3,4$. The stratification is weak so the two layers are strongly coupled. The lower layer contour has similar evolution as seen in Figure 4.3 for the one-layer model run with $h_{0} / \epsilon=5$. 
and has only order Rossby number penetration into the upper layer. The restrictions of this model have been discussed earlier, and the steady model has been shown to have dynamics consistent with the approximations. Time dependent solutions for this geometry are compared with those for flow over small topography in Section 4.4 so that the eddy shedding process in a stratified fluid can be better understood. The method of solution used in this problem is similar to that for the problem of barotropic flow over finite topography (Section 4.3). Once again, the problem is solved in two regions and then a zero potential vorticity solution is used to apply the matching and boundary conditions. We concentrate on the two values for the stratification used in Section 4.4 .

\subsubsection{Numerical Method}

In this model, the layer depths are changed by only an order Rossby number amount, both over the topography and away from the topography. Therefore, the magnitude of the induced cyclonic and anticyclonic circulations is the same. We independently solve the contour dynamics problem in the two regions (over the topography and away from the topography) and then use the zero potential vorticity solution to match the two regions together. In the interior region, the fluid satisfies the one-layer potential vorticity equation, while, in the outer region, the fluid satisfies the two layer equation. Therefore, the form of the Green's functions as well as the zero potential vorticity equations are different in the two regions.

In the inner region, the Green's function is simply the one-layer logarithmic Green's function given in 4.3. In the outer region, the Green's function is similar to the Green's function used in 4.18 and 4.19 , but this time the potential vorticity anomaly is in the upper layer. Therefore, the Green's functions needed in the outer 
region are

$$
G_{11}=\frac{\delta}{1+\delta} \ln R-\frac{1}{1+\delta} K_{0}(\Gamma R)
$$

and

$$
G_{12}=\frac{\delta}{1+\delta} \ln R-\frac{\delta}{1+\delta} K_{0}(\Gamma R)
$$

The zero potential vorticity solution is found as in Section 4.3 by applying matching conditions in addition to boundary conditions applicable to the geometry of the problem. The contribution to the velocity from the contour integral is found at $r=1$ for the upper and lower layers in the outer region $(r>1)$ and for the upper layer in the inner region $(r<1)$. The velocity is then transformed into cylindrical coordinates, and application of a Fast Fourier Transform decomposes the velocity into a sum of modes in $\theta$. Thus at $r=1$, the edge of the topography, the velocity can be written in the outer region as

$$
\begin{aligned}
& u_{j}(\theta)=\sum_{n=-N / 2}^{N / 2} u_{n}^{j} e^{-i n \theta} \\
& v_{j}(\theta)=\sum_{n=-N / 2}^{N / 2} v_{n}^{j} e^{-i n \theta}
\end{aligned}
$$

where $j$ delineates the layer and

$$
\begin{aligned}
& u(\theta)=\sum_{n=-N / 2}^{N / 2} u_{n}^{i} e^{-i n \theta} \\
& v(\theta)=\sum_{n=-N / 2}^{N / 2} v_{n}^{i} e^{-i n \theta}
\end{aligned}
$$

for the velocity in the inner region (where now $u$ is the radial velocity and $v$ is the azimuthal velocity).

The homogeneous solutions are written

$$
\psi_{1}^{h}=\sum_{n=-N / 2}^{N / 2} a_{n} K_{|n|}(\Gamma r) e^{-i n \theta}+b_{n} r^{-|n|} e^{i n \theta}+b \ln r
$$


and

$$
\psi_{2}^{h}=\sum_{n=-N / 2}^{N / 2}-\delta a_{n} K_{|n|}(\Gamma r) e^{-i n \theta}+b_{n} r^{-|n|} e^{i n \theta}+b \ln r
$$

for $r>1$ and

$$
\psi_{1}^{h}=\sum_{n=-N / 2}^{N / 2} c_{n} r^{|n|} e^{i n \theta}
$$

for $r<1$.

The boundary conditions at the topography in the lower layer are satisfied independently for each mode. For no flow normal to the boundary in the lower layer we have

$$
i n\left(a_{n}-\delta b_{n} K_{n}\right)+u_{n}^{2}=0 .
$$

We have to choose the circulation around the topography in the lower layer, and as we did in the steady solution we choose for there to be no circulation. This condition gives

$$
-\delta \Gamma K_{0}^{\prime}(\Gamma)+b+v_{0}^{2}=0 .
$$

The velocity in both directions must match in the upper layer. These conditions are satisfied when

$$
u_{n}^{i}+i n c_{n}=i n\left(a_{n}+b_{n} K_{n}(\Gamma)\right)+u_{n}^{1}
$$

and

$$
v_{n}^{i}+n c_{n}=-n a_{n}+\Gamma b_{n} K_{n}^{\prime}(\Gamma)+b \delta_{n o}+v_{n}^{1} .
$$

We note that for a real solution $a_{n}=a_{-n}^{*}$ and $b_{n}=b_{-n}^{*}$. For $n=0$, there is no contribution from the contour integral so that $u_{0}^{i}=u_{0}^{1}=0$, because the radial velocity is given by $u=-\frac{\psi_{\theta}}{r}$ and the lowest order mode in $\theta$ of this is zero. The coefficients in $4.28,4.29$, and 4.30 are solved for from $4.31,4.32,4.33$ and 4.34 . In practice, 16 modes in $\theta$ were sufficient. As in Section 4.3, the vorticity is conserved using this method.

After each numerical experiment, the height of the interface must be checked to make sure that it does not go above the topography, because if it does, the model 
dynamics no longer make sense. The interface can be calculated everywhere, using the lower layer potential vorticity, as in Section 4.3. In this case, the lower layer potential vorticity is zero everywhere. The interface height is given by 4.16 with $h=0$, and can be found by calculating the relative vorticity everywhere,

$$
\eta=\frac{\nabla^{2} \psi_{2}}{1+\delta}
$$

similar to 4.20. The relative vorticity is calculated in two parts. First the contribution from the zero potential vorticity solutions is determined analytically from the series expansion, which is equivalent to calculating $\eta$ directly from the homogeneous series solution from the stream function in both layers. Next, the contribution from the contour integral is calculated by taking derivatives of the contour integral in the same way as in 4.21 . For the examples shown below, the interface does not go above the topography. We can get a qualitative idea of when the interface would go above the topography by looking at Figure 3.19 which gives the critical height above which we expect the interface to go above the topography in the steady solution.

\subsubsection{Numerical solutions}

From the steady solutions, we predict that decreasing the stratification will result in more fluid remaining over the topography in the initial value problem (see Figure 3.20). In this model there is no fluid that originates over the topography in the lower layer so we only show the contour that marks fluid that originated over the topography in the upper layer. Thus a smaller volume of fluid is initially over the topography than in the solutions shown in Section 4.4. Keeping this in mind, we proceed to compare the two solutions.

When the stratification is strong, the interface is rigid and the layers are uncoupled (Figure 4.21). The development of the contour is nearly identical to that seen in both Figures 4.3 and 4.15, and the upper layer response of this model is the 
same as the lower layer response of the small topography model. The layer depths are equal so the fraction of the upper layer taken up by the topography matches the fractional extent of the topography in the lower layer in the small topography model.

When the stratification is weak, the amount of fluid that remains over the topography is increased, as expected from the results of Section 3.3 and seen in Figure 3.20, (Figure 4.22). The total velocity field shows that the shed eddy is nearly barotropic but slightly surface intensified while the flow near the topography is baroclinic (Figure 4.23). This response is due to the requirement that all of the flow go around the topography in the lower layer, while some of the fluid goes over the topography in the upper layer.

The interface is depressed near the topography and raised over the eddy (Figure 4.24) opposite to what is seen in Figure 4.18. A cut along the $x$ axis is made to see the time-evolution of the interface ( Figure 4.25). Initially, the interface is flat. Then the interface is raised near the topography when the fluid which originated over the topography is partially on and partially off the topography. As this fluid moves downstream, the interface becomes depressed around the topography as it responds to the anticyclonic potential vorticity anomaly in the upper layer, and the shed eddy appears downstream of the topography as a positive perturbation which is advected away. The interface is raised over the shed cyclonic eddy as would be expected to compensate for the potential vorticity anomaly (Figure 4.25). However, we point out again that this result that the shed eddy is cold core is true only because of the detailed structure of the stratification in this model. One would expect that the shed eddy would be cold core in a continuously stratified model only if the stratification were strong near the region of the interface, and relatively weak above that. If the fluid were strongly stratified above the interface, then the shed eddy would have a more complicated vertical structure. It would probably have a cold relative to the external fluid in its deepest level a warm anamoly in its shallowest level. In both 

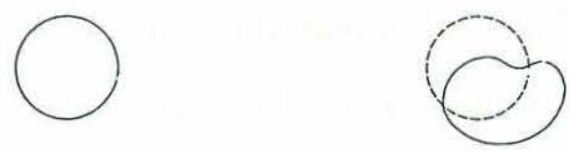

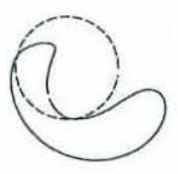

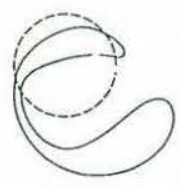

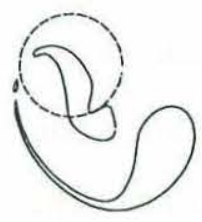

Figure 4.21: Time evolution of the contour that delineates fluid that originated over the topography in the upper layer in the two-layer finite topography model. $\Gamma=0.25$, $\delta=1$ and $h_{0} / \epsilon=2.5, t=0,1,2,3,4$. The stratification is strong so the two layers are relatively uncoupled. Notice the similarity of this figure to Figure 4.3 and Figure 4.15 .

186 

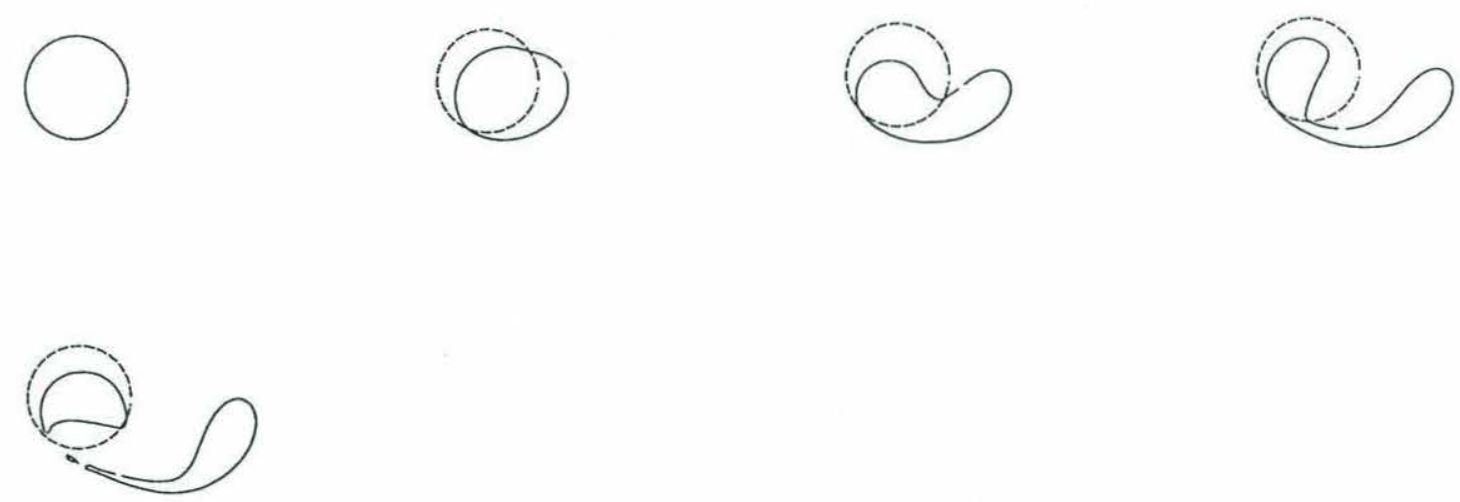

Figure 4.22: Time evolution of the contour that delineates fluid that originated over the topography in the upper layer in the two-layer finite topography model. $\Gamma=4$, $\delta=1, h_{0} / \epsilon=2.5$, and $t=0,1,2,3,4$. The stratification is weak so the two layers are strongly coupled. More fluid is trapped over the topography than in Figure 4.21. 


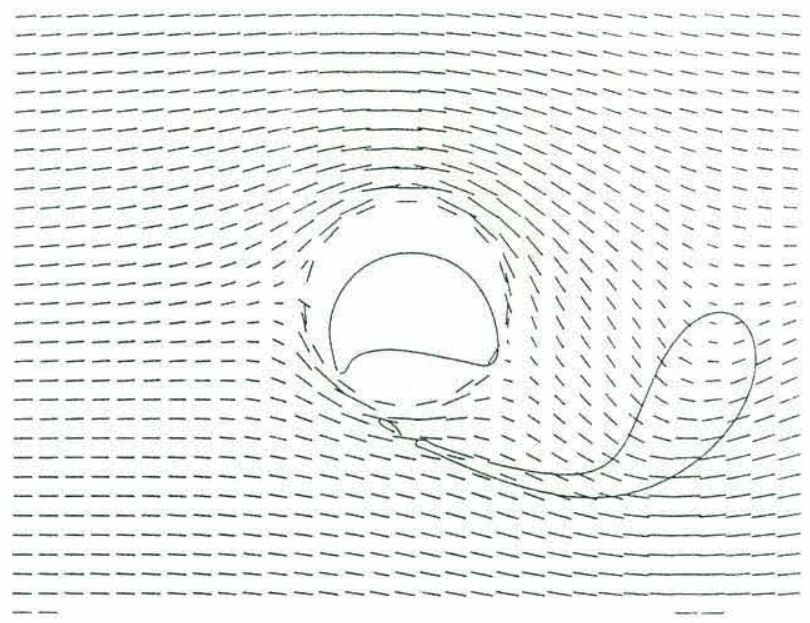

(a)

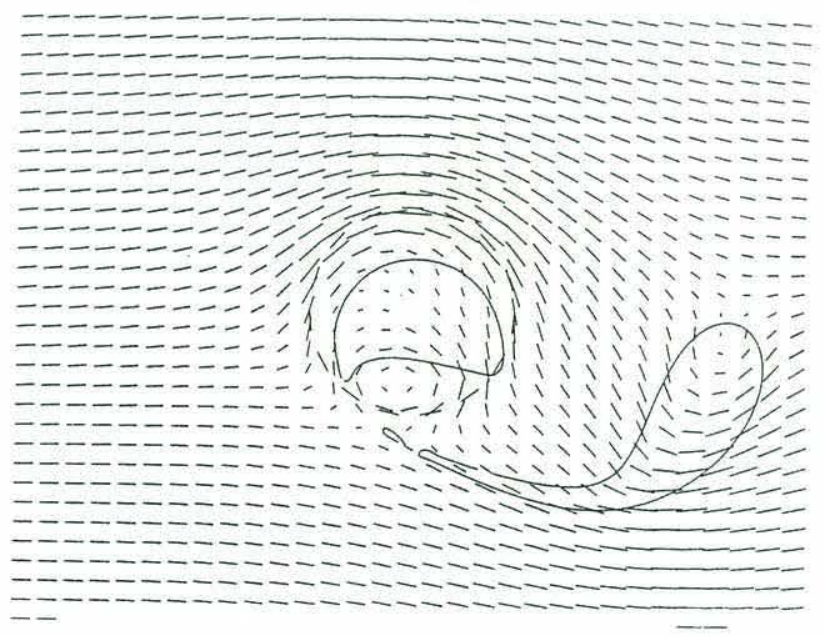

(b)

Figure 4.23: The flow field for the case shown in 4.22 at $t=4$. (a) Lower layer and (b) upper layer. The lower layer flow is forced to go around the topography. The shed eddy has cyclonic circulation, while the flow over the topography is anticyclonic. 
of the two-layer models, the interface deflects to make the layer depth smaller to compensate for the positive relative vorticity in the fluid shed from the topography. The cut in Figure 4.25 is not made through the center of the eddy so that the interface excursion due to the eddy is higher than shown. Also, the height of the topography is 2.5 in the same units as the interface, above the height of the interface at the boundary of the bump at $r=1$. This numerical run is in a physically realizable regime.

The examples shown above all are done with $\delta=1$, which allows comparison of the time evolution of the flow in this model with the two-layer model over small topography. However, in the ocean, a more typical value for $\delta$ would be 0.2 , so to make qualitative predictions with our model, we do two models runs with $\delta=0.2$. To compare with the previous runs, we first let the potential vorticity anomaly be the same as the example shown in Figure 4.22. In the second run we keep $h_{0} / \epsilon$ the same as in Figure 4.22.

The potential vorticity anomaly is given by $q_{a}=h_{0}(1+\delta) / \epsilon \delta$ which is the amount of relative vorticity generated when the fluid that originates over the topography moves off the topography. In the generic case shown in Figure $4.22, q_{a}=5$. In Figure 4.26, we show the evolution of the contour when we use the same value of $q_{a}$, but this time $\delta=0.2$, so that the topographic height in the upper layer is smaller relative to the total depth of the fluid. The topographic height in the upper layer relative to the upper layer depth is the same in the two runs. The development of the flow field in Figure 4.26 is quite similar to that in Figure 4.22. We showed in the steady solutions that when the lower layer thickness is larger, the flow in the lower layer tends to be more symmetric about the $x$ axis. There are two obvious differences in the two time-dependent runs; first, the trapped eddy is not rotated as far in Figure 4.26 as in Figure 4.22, and second, the shed eddy is shed closer to the $x$ axis. The shed eddy in this run is caught up in the island-like flow which is quite important 


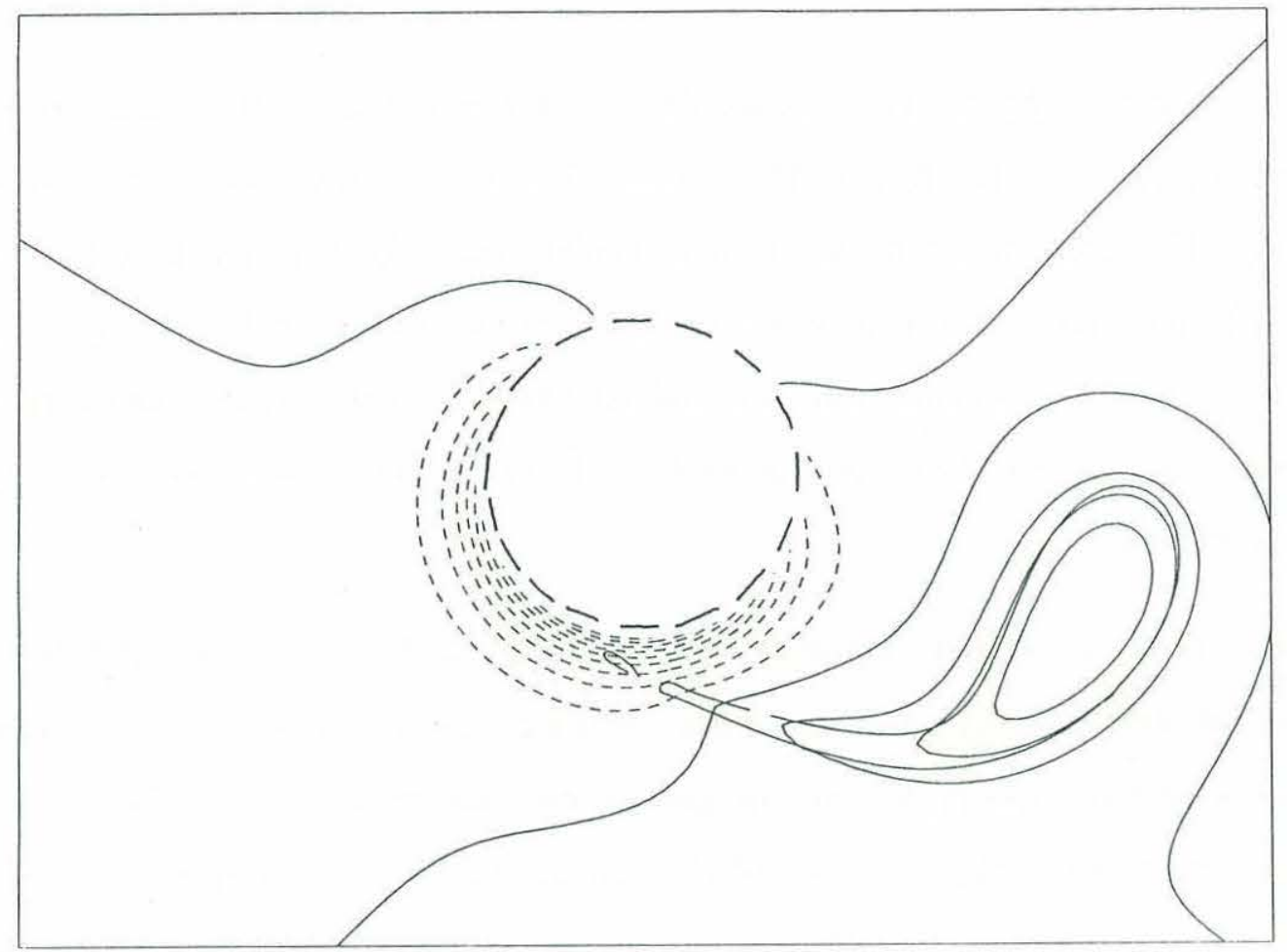

Figure 4.24: The interface displacement for the case shown in Figure 4.23 at $t=4$. Dashed contours indicate negative interface displacement. The interface is depressed near the topography and is raised over the shed eddy. The shed eddy is cold core, cyclonic and surface trapped. The solid line outside of $r=1$ delineates fluid which originated over the topography in the upper layer. 


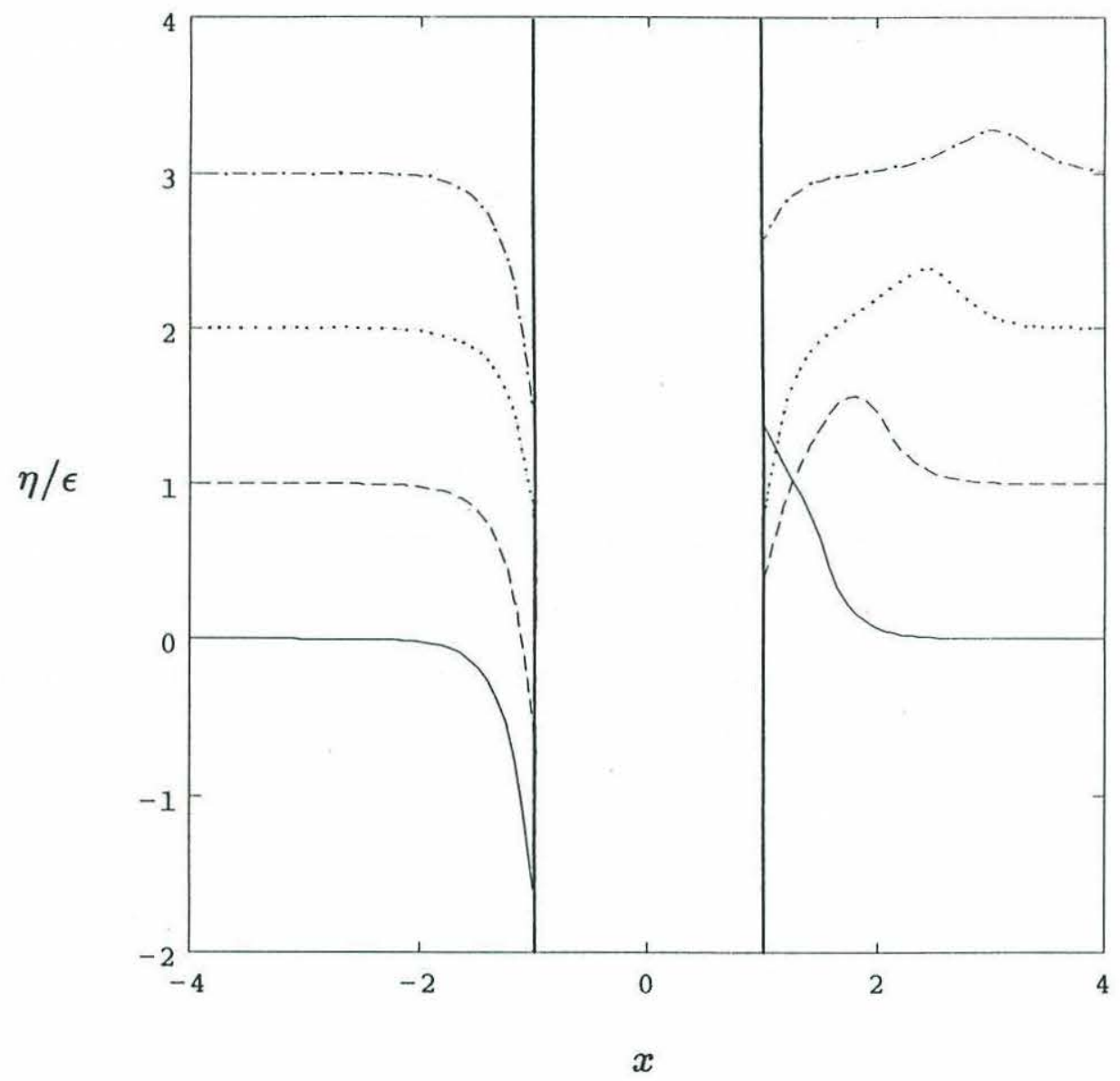

Figure 4.25: The interface displacement for the case shown in Figure 4.22 at a cut along the $x$ axis at $t=1,2,3,4$. The height of the topography in the same units is 2.5. The interface is depressed near the topography and is raised over the shed eddy. The shed eddy is cold core. Notice that the cut is not taken through the center of the eddy, so that the maximum interface displacement due to the shed eddy is larger than shown. The interface is offset by 1 unit at $t=2$, by 2 at $t=3$, and by 3 at $t=4$. 
close to the topography and is advected away more quickly than in Figure 4.22. The similarity of the contour evolution between the two runs shows that the potential vorticity anomaly dominates the flow evolution, not the height of the topography in the upper layer. It is important to remember that the total topographic height in Figure 4.26 in this run is an order one amount larger than that in the run in Figure 4.22 .

In the second run with $\delta=0.2$, we let the topographic perturbation be the same as the run in Figure 4.22. In this case, more fluid is trapped over the topography because the potential vorticity anomaly is larger in this case (Figure 4.27), and the topographic height relative to the upper layer thickness is larger. The fluid shed off the topography is released close to the $x$ axis. In the lower layer, the flow is similar to that of irrotational flow about a cylinder, and this flow couples to the upper layer so that the shed eddy is quickly swept away close to the $x$ axis. It is important that the area of trapped fluid over the topography is larger in this run, but since the layer thickness is substantially less, the total volume of fluid that remains over the topography is actually smaller than in Figure 4.22 .

\subsubsection{Summary: Flow over topography in a stratified model}

The two models above illuminate the basic characteristics of flow over topography in a stratified model. In particular, the difference in the eddy generation process between flow over topography when all of the isopycnals flow over the topography, and flow for which an isopycnal intersects the topography has been explored. The steady solutions presented in Section 3.3 help in the understanding of the process. The interface displacement is calculated, and this quantity can be easily compared to calculations done in more complicated numerical models. The finite topography model is potentially more relevant to flow in the ocean because it allows the interface 

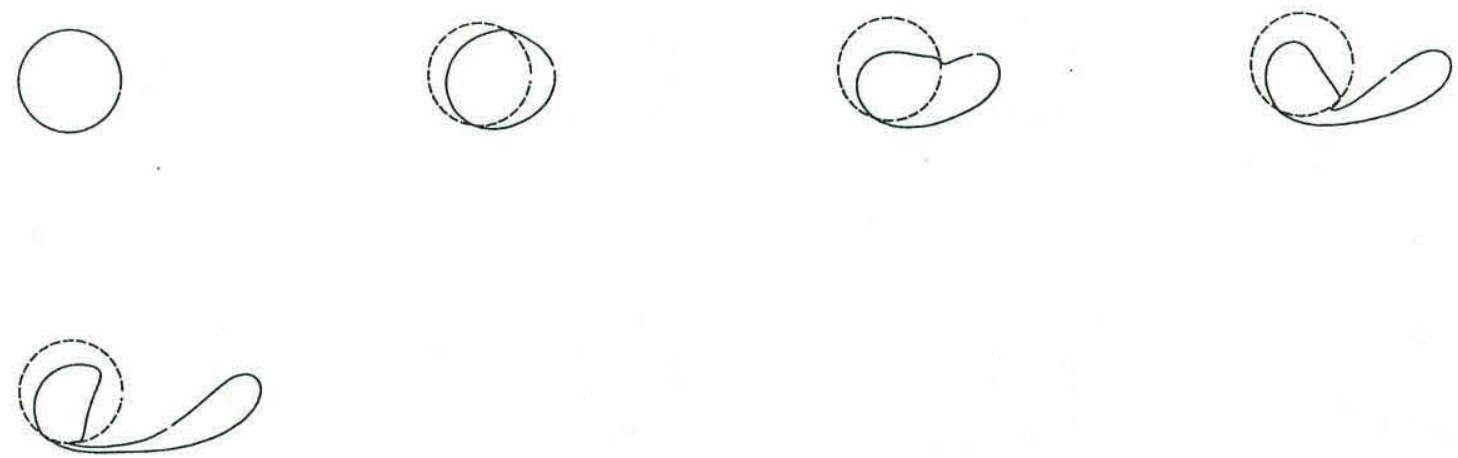

Figure 4.26: Time evolution of the contour that delineates fluid which originated over the topography in the upper layer in the two-layer finite topography model. $\Gamma=4$, $\delta=0.2$ and $h_{0} / \epsilon=0.833$. This run has the same potential vorticity anomaly as in Figure 4.22. 

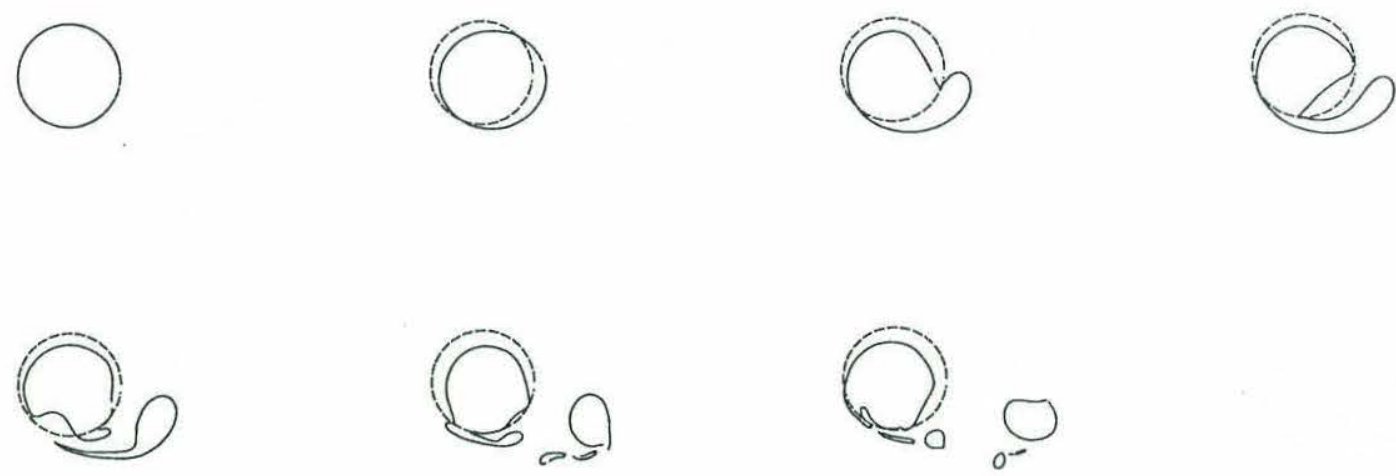

Figure 4.27: Time evolution of the contour that delineates fluid which originated over the topography in the upper layer in the two-layer finite topography model. $\Gamma=4$, $\delta=0.2$ and $h_{0} / \epsilon=2.5$. Successive eddies are shed by the topography. 
to intersect the topography. Although the quasi-geostrophic model is limited in application, the dynamics in this model could be important in the ocean. We choose not to try and make predictions of oceanic flow from this idealized model since we have left out many important effects, including continuous stratification and vertical shear, but rather explore the parameter dependence of the models and understand qualitatively the evolution of the shed cyclonic eddy and trapped anticyclonic eddy and how this evolution differs in the two-layer small topography model. We choose to concentrate on two values of stratification to illustrate the dynamics of the models: strong stratification in which the internal Rossby radius of deformation is 4 times the length scale of the topography, and weak stratification in which the internal Rossby radius of deformation is one quarter the length scale of the topography.

When the topography is small, the shed eddy is warm core and bottom trapped. When the topography is finite, the shed eddy is cold core and surface intensified. As predicted from the steady solution in Chapter 3 , as the stratification increases, less fluid remains over the topography in the initial value problem in the finite depth model while more fluid remains over the topography in the small bump model. As the lower layer depth increases, more fluid remains over the topography in the finite depth model. The parameter that determines the amount of fluid remaining is the topographic height in the upper layer relative to the upper layer thickness, even when the stratification is relatively weak. The steady solutions do well in predicting what parameters will be important in the flow evolution, but cannot predict the final steady state of the system.

The two models shows the two classes of quasi-geostrophic solutions which are consistent with the velocity remaining approximately horizontal. If we were to work with a primitive equation model with continuous stratification we would expect to see a combination of the dynamical processes seen in the two types of solutions described in this section. We speculate that a primitive equation model would allow exploration 
of parameters such that a smooth transition between solutions of the types described could be found.

\subsection{Summary and conclusions}

In this chapter we study eddy generation by flow over topography in four different models as sketched in Figure 1.1. First, we consider flow over topography in one layer and find that the amount of fluid that remains over the topography increases as we increase the time over which the background flow is turned on. This suggests that the solution from the initial value problem is identical to the maximum retention solution when the turn on time of the background flow becomes very large, while the intermediate retention solutions are found as the quasi-steady state solution when the velocity is turned on more quickly. The ejection of blobs of fluid is an important process in reaching the quasi-steady state; to reach a steady state friction is

needed. A comparison between possible steady state solutions and the final time step of the initial value problem shows the relationship between them. In the initial value problem, the fluid that is trapped within closed streamlines near the topography is composed of fluid which originated over the topography and fluid which originated upstream.

We consider the flow over finite topography in one layer. The development of the flow is different depending on the height of the topography. Each run is compared with the quasi-geostrophic simulation. For small topography $\left(h_{0}=0.25\right)$ the finite depth solutions show more rapid development of the flow over the topography due to the enhancement of the flow there. As $h_{0}$ is increased, the eddy shedding process changes, such that two cyclonic eddies are created when $h_{0}=0.5$, and finally, when the topography fills up most of the water column, the eddy remains trapped over the 
topography and oscillates on and off the topography while rotating clockwise around it.

Because stratification is important in the ocean we consider flow in two twolayer models. First we consider flow over small topography. In this model, a warm core, bottom trapped, cyclonic eddy is shed, with more fluid remaining over the topography with increasing stratification. The results of the model are similar to those found by Huppert and Bryan (1976) in a study of flow in a primitive equation model over small topography. We concentrate on two values of stratification to illuminate the dynamics of the model and compare the results to the one-layer model results. We extend this model to include the effect of finite topography, allowing the interface to intersect the topography. In this quasi-geostrophic model a cyclonic, surface intensified, cold-core eddy is shed and more fluid remains over the topography with decreasing stratification. We concentrate on the same two values of stratification and show how the dynamics are controlled by the lower layer depth. The steady solutions in Chapter 3 provide a guide in the study of the time-dependent solutions, although they are not good predictors of the final steady state solutions when fluid remains trapped over the topography.

The finite depth model allows exploration of a previously unexplored parameter range. This model requires that the Rossby number be small and the stratification strong enough such that isopycnals do not climb up over the topography. In the finite depth model, we must choose the circulation in the lower layer to complete the solution. We choose the circulation to remain zero for all time in this initial value problem. This choice affects the dynamics of the flow. In particular, instead of the interface being raised near the topography, which one would have predicted from previous work, the interface is low near the topography. The structure of the shed eddy is independent of this because it depends locally on its potential vorticity structure which would not be affected by the circulation around the topography. 
The two finite-depth models require the development of a modified contour dynamics method. This method has more general applications than has been explored here. For instance, one could consider elliptical topography instead of circular topography, straight line topography or the interaction of constant potential vorticity patches with islands as briefly described in Appendix C. 


\section{Chapter 5}

\section{Conclusions}

\subsection{A Summary of the thesis}

In this thesis we describe flow over isolated topography using several different models in an attempt to gain understanding of what occurs when a uniform flow field interacts with finite topography. We begin by studying the structure of stagnant Taylor columns on the $\beta$-plane. These solutions are valid for a time scale which is comparable to the spin-up time. We move beyond quasi-geostrophic theory and find steady solutions for flow over finite topography in a one-layer and a two-layer model. When closed streamlines form these solutions are valid for time scales short compared to the spin-up time and do not include frictional effects. We finally consider the time-dependent problem of flow initiation over topography in both one and two-layer models and look at the flow over the advective time scales. The results of each of these studies are discussed below.

In Chapter 2 we find stagnant Taylor column solutions for flow on the $\beta$ plane of the type that Ingersoll (1969) found for flow on the f-plane. In this quasigeostrophic model, the topography is large enough such that closed streamlines form in the fluid. Within closed streamlines the flow is stagnant. We use an iterative 
boundary integral technique related to contour dynamics. Because we have developed a general technique, the solutions that we find do not require that an analytic solution of the problem be found. To test the numerical method, we reproduce Ingersoll's (1969) solution for flow on the f-plane over a right circular cylinder in which the Taylor column is contained within a circle. We find that the Taylor column becomes elongated perpendicular to the flow direction when the topography is a cone, giving the Taylor column a tear-drop shape. We suggested that this shape is representative of what may be found for flow over other monotonic axisymmetric obstacles. The solution is also found for flow over a right circular cylinder on the $\beta$-plane. For westward flow, the Taylor column becomes elongated parallel to the flow and is trapped toward the northern portion of the topography. For eastward flow, the situation is quite complicated because of the generation of stationary Rossby waves downstream of the topography. The Taylor column is slightly elongated perpendicular to the background flow. However, closed streamlines can appear downstream of the topography. It was not possible using our numerical technique to find consistent solutions such that these regions were also spun down. Experience with the numerical method suggests that the stagnation point of the Taylor column always lies on the boundary of the topography, where the height goes to zero. We infer from this it may not be possible to find the stagnant Taylor column for flow over topography which does not have compact support. Additional solutions to the inviscid problem appear in the process of finding the frictional solution. We call these intermediate retention solutions. There is a patch of fluid which is stagnant, but it is contained within additional closed streamlines. These solutions are valid for time scales short compare to the spin up time.

In Chapter 3, steady flow over finite topography is considered. In the first part of the chapter, solutions are found for flow in a barotropic fluid. These solutions are extensions to those found by Bannon (1980) for flow over a Gaussian obstacle in the shallow water model. Because we consider flow over the right circular cylin- 
der, analytic solutions are possible and a more extensive comparison can be made between the quasi-geostrophic and finite depth solutions. The flow field for the finite depth solution shows marked differences from the quasi-geostrophic solution which are illustrated by comparing the solutions themselves as well as by comparing global quantities calculated from the stream function. The solution can be divided into two parts: the topographic component (completely even in $y$ ) that is forced by the potential vorticity anomaly caused by vortex squashing and is similar to the quasigeostrophic solution, and the island component (completely odd in $y$ ) which is forced by the application of the matching conditions at the boundary of the topography. In the quasi-geostrophic solution the odd part is simply the background flow. The finite depth model allows several interesting features when the effect of $\beta$ is strong. The transport is increased over the topography relative to the background flow for both eastward and westward flow in this regime, a reflection of the tendency for streamlines to follow lines of constant background potential vorticity when the $\beta$-effect is large. For eastward flow, the drag can be an order of magnitude larger than that predicted in quasi-geostrophic theory due to the quadratic dependence of the pressure on the velocity found through the conservation of the Bernoulli function. The model has some physical limitations which are discussed further in Appendix B.

In the second part of Chapter 3 we discuss flow over finite topography in a stratified two-layer model in which the interface intersects the topography. This model contains a new parameter regime in which quasi-geostrophic dynamics are consistently applied, but the topography has order one height. This model is an extension of Buzzi and Speranza's (1979) work. They considered quasi-geostrophic flow near two different obstacle shapes in a linearly stratified fluid, a small (order Rossby number) hemisphere, and vertical plate that obstruct the basic flow. The bottom is an isopycnal surface in the first example, and in the second example, isopycnals intersect the topography so that flow goes around the obstacle in the lowest levels. In their model, at a wall, the stream function depends only on the 
vertical coordinate and its functional dependence is chosen arbitrarily. In our model, we consider an obstacle that has both a finite horizontal cross-section as in their first example, and a finite vertical cross-section as in their second example, and we must choose the circulation around the topography in the lower layer. We obtain a solution which contains dynamics consistent with both examples. As in the barotropic finite depth model, the flow can be divided into an odd component in $y$ (driven by the wall like character of the obstacle) and an even component in $y$ (driven by the potential vorticity anomaly over the topography in the upper layer). The fluid in the lower layer is required to go around the topography but that does not necessarily mean that closed streamlines have formed in the flow. We choose to consider solutions in which the circulation in the lower layer is zero, as is consistent with the solution being the steady state of an initial value problem where there is no flow anywhere initially.

Fluid is more easily trapped in the upper layer when the stratification is weak; in a two-layer model of flow over small topography there is more trapped fluid in the lower layer when the stratification is strong. When the stratification is weak, the upper layer is strongly coupled to the lower layer and the effective height of the topography is larger in the finite depth model. In the small topography model of McCartney (1975) when the stratification is weak, the vertical penetration scale is larger so that the effect of the topography is spread throughout more of the water column. The model breaks down when the interface goes above the topography, which occurs more easily for weaker stratification. The interface is depressed near the southern part of the topography, and raised in the north. This effect is enhanced for background flows with vertical shear whereas in the small topography model the interface is raised as an axisymmetric bump over the topography. Examples are also shown with the effects of $\beta$ included.

In Chapter 4, we consider the initial value problem of flow over topography on the f-plane. We restrict ourselves to flow on the f-plane so that we can use the method 
of contour dynamics to isolate the effects of the time-dependence of the background flow, the finite topography, and stratification on the shedding and trapping of inviscid eddies over the topography. First we examine flow over quasi-geostrophic topography and show that the time-dependence of the background flow is an important factor in determining how much of the fluid that originated over the topography remains in the initial value problem. We suggest that Johnson's (1983) maximum retention solution in which the maximum amount of fluid that originated over the topography remains there can be realized when the flow is turned on smoothly over a long period of time. We construct a family of steady solutions in which only part of the fluid that originated over the topography is retained there, and we suggest that one of these solutions is obtained as a quasi-steady state in the initial value problem.

We then consider the initial value problem in the finite depth model. A new method is developed to apply boundary conditions at circular boundaries, extending the range of problems that can be considered with the method of contour dynamics. We solve the problem in the two regions over the topography and away from the topography separately using traditional contour dynamics, and then construct zero potential vorticity solutions to match the two regions. Because there is a change in the symmetry of the steady solution when the topography is finite and the flow is horizontally divergent, the time-dependent solutions for flow over finite topography in a barotropic fluid look quite different from quasi-geostrophic solutions. Two new dynamical regimes are illustrated. When the topographic height is half of the depth of the fluid, two cyclonic inviscid eddies are created, instead of only one as seen in the quasi-geostrophic model. When the topography takes up most of the water column, the fluid oscillates on and off the topography, moving clockwise around the topography without shedding any fluid in what appears to be a stable oscillation.

The eddy shedding process in the two-layer model is considered so that the effect of stratification can be illustrated. When the topography has only an order 
Rossby number height, the shed eddy is cyclonic, warm-core, and bottom-trapped. In contrast, in the finite topography model, the shed eddy is cold-core, cyclonic and surface-intensified, which can be seen by calculating the interface height. The difference between the results from the finite depth and small topography steady solution provides a guide for understanding the results of the time-dependent experiments. The parameter that effects the development of the flow in the finite depth model is the ratio of the height of the topography in the upper layer to the upper layer depth. The finite depth two-layer model is shown to have dynamics consistent with the approximations and illustrates a new dynamical regime in the study of eddy shedding off topography that has not been considered before.

In synthesizing the results in the Chapters 2,3 , and 4 it becomes clear that the solution of flow near topography depends in detail on the potential vorticity and thus the history of the fluid parcels that end up trapped over the topography. We have studied three different types of solutions that are relevant for three different time scales. In Chapter 2, the potential vorticity of the trapped fluid is zero, and the fluid parcels were assumed to have originated upstream, but their relative vorticity is lost through the action of bottom friction. In Chapter 3 , the fluid within closed streamlines originated upstream and its potential vorticity is determined by upstream characteristics. Finally, in Chapter 4 we study solutions in which within closed streamlines some of the fluid parcels originated over the topography with relative vorticity of zero, while other fluid parcels originated upstream. We show that the amount of either type of fluid that is trapped over the topography depends on the time history of the background flow and on the point in time that we choose to look at the solution. 


\subsection{Advantages and limitations of the models}

Throughout this thesis, inviscid, non-linear dynamics is of order one importance, and the flow is solved for over an infinite domain so that numerical friction, radiation conditions, channel walls, or periodic boundary conditions do not affect the solutions.

The study in Chapter 2 helps to answer the question as to what determines the shape of Taylor columns in a barotropic fluid. Because all of the solutions to this problem that have been found have the stagnation point of the Taylor column on the edge of the boundary, we conjecture that it is not possible to find a solution for smooth topography, but we have been unable to prove that this is true. That we were unable to find solution for smooth topography could be, however, because the technique that we use is not suitable for the problem of finding the shape of a Taylor column when the topography is not compact. Also, it is not clear at this point how one would go about extending the criterion that Ingersoll (1969) developed for the conditions at the boundary of the Taylor column to either a layered model or a continuously stratified model. For this reason, as well as the large time-dependence in actual oceanic flow these solutions may have limited application to real oceanographic situations.

The finite depth models studied in Chapter 3 have the advantage of allowing analytic solutions to be found, so that a thorough exploration of parameter space is possible, and new parameter regimes are examined. The barotropic model allows consideration of the interaction of the effect of finite topography and the effect of $\beta$. We have already discussed some of the physical limitations of this model. In particular, the vertical velocity is infinite along the boundary of the topography in this model due to the fact that there are no vertical variations in the horizontal velocity, and the pressure is discontinuous along at the edge of the topography. These problems are discussed further in Appendix B. The finite step in the topography also introduces 
resonant Rossby waves, making the drag fall off more slowly than expected from the results of models of flow over smooth topography, and the Rossby waves downstream of the topography can form closed streamlines, making the steady solutions suspect. These limitations probably do not affect the qualitative results of the analysis.

The two-layer finite depth model extends quasi-geostrophic theory to a new regime in which the isopycnal intersects the topography. The geometry of this model is quite special but gives reasonable qualitative results and demonstrates a parameter regime that has been unexplored. Because the geometry requires that the lower layer depth and the topographic height be approximately the same, the solutions are not very general, and a different model must be used to consider cases in which the topographic height and the lower layer depth are determined separately. The calibration of the model in terms of a continuously stratified ocean is unclear, so the appropriate values for the parameters in the model to correspond to a realistic oceanic situation are unknown. In particular, we expect that the model would be relevant to situations in which the stratification is localized in the vertical, and above the region of strong stratification, the fluid is reasonably barotropic. If the structure of the stratification were different, we expect that the flow would be qualitatively different from what we foun in this simple two-layer model..

In Chapter 4 we consider the time-dependent eddy shedding process in an infinite domain so that unlike most other numerical solutions we do not have to worry about the effect of channel walls or radiation conditions. In addition, the method of contour dynamics is not computationally intensive. We are of course restricted to considering only obstacles that have depths which are piecewise constant, flow on the f-plane, and flow with no background vertical shear. This last requirement is probably quite restrictive for flow in the the ocean where the flow tends to be surface-intensified. However, we do expect that the qualitative results are robust. We have developed a 
modification to contour dynamics so that boundary and matching conditions can be applied.

The effect of finite friction cannot be considered directly with these models. We can, however, make some guesses as to what its effect would be. We would expect that friction would reduce the strength of the shed eddy and the flow within the shed eddy would weaken with time. Friction could also effect the circulation in the lower layer so that it could change in time.

\subsection{Relevance of modeling results to the ocean}

There are several qualitative results contained in this thesis that may help with the interpretation of oceanic observations and more complicated model results.

The results of Chapter 2 suggest that for topography that monotonically increases in height the Taylor column would be stretched in the direction perpendicular to the incoming flow, and would be narrower near the stagnation point located on the right hand side of the topography looking downstream. For westward flow, the Taylor columns would be elongated parallel to the incoming flow. These steady solutions are set up over the spin up time of the fluid and may be qualitatively correct under situations in which the background flow is fairly steady.

The steady solutions over finite topography in the barotropic model discussed in Chapter 3 show that quasi-geostrophic solutions may vastly underestimate the drag that the topography exerts on the flow even for relatively small Rossby numbers and that topography with relatively large horizontal extent (large $\beta$-effect) exerts a large amount of drag on the background flow field.

The two-layer finite topography model demonstrates that it is likely that flow will go around tall bathymetric features in the lower levels, and over them in upper 
levels. We see evidence of this in the study done by Vastano and Warren (1976), where the lines of constant potential density tend to be split by the topography in the lowest levels, while going over the topography in the upper levels. Also, for finite topography, one expects that the isotherms will tend to be lower on the right side of the topography looking downstream than on the left hand size, and that vertical shear would enhance this effect. These displacements may be evident in some of the density sections of Roden and Taft (1985) although the time-variability in the region of study makes interpretation of the flow field in terms of a steady model difficult.

The eddy shedding studies are also relevant to oceanographic features because of the highly variable flow fields in the ocean. The experiments done in the onelayer model by varying the turn-on time of the background flow suggest fluid can be trapped over the topography in the initial value problem and that this fluid can remain over the topography for all time. The amount of trapped fluid also depends critically on how the background flow is initiated, so the flow field in regions of the ocean where the ocean is swiftly changing from one background flow regime to another would look different from a place where the flow is changing more slowly. It is not clear which way of turning on the flow, whether quickly or slowly, is more relevant to the oceanographic problem. In regions such as the Gulf Stream, the transverse location of the jet can change rapidly, resulting in a highly variable background flow field over the New England Seamounts.

In the finite depth experiments a cold-core eddy that is surface trapped is shed. This is in contrast to traditional quasi-geostrophic theory in which the shed eddy is warm core and bottom trapped. This suggests a way in which cold-core cyclonic eddies could be generated in the ocean and variability might be introduced into the upper levels of the ocean by the interaction of flow with topography. For instance, Roden and Taft (1985) study the region in which the Kuroshio crosses the Emperor Seamounts. There is clear enhanced variability near the surface. This could 
be caused by the mechanism described in our two-layer model, or by the surface intensification of the Kuroshio.

We have not tried to make detailed predictions with the models in this thesis, partly because we have left out some important effects in our search for problems that are both easy to pose and tractable, but also because it became clear during the modeling that the detailed history of the flow field must be known in order to make detailed predictions about the evolving state of the flow field.

\subsection{Future work}

The modeling done in Chapter 4 required the development of a modification to the traditional contour dynamics method allowing boundary conditions to be applied on solid boundaries. Appendix $\mathrm{C}$ outlines the application of the method to the problem of a vortex interacting with an island, and this problem deserves more of an exploration that was given here and merits an extension to a two-layer model

. There are two more extensions to the time-dependent modeling that should give results that would be important for the interpretation of oceanic flow. The first is the study of the interaction of an isolated jet with topography. This could be approached initially with the one-layer quasi-geostrophic model. The second is the modeling of the interaction of flow with more than one topographic feature. Both of these have application in the study of the Gulf Stream as it crosses the New England Seamounts. 


\section{REFERENCES}

Batchelor, G.D. (1967) An Introduction to Fluid Dynamics, Cambridge University press.

Bannon, P.R. (1980) Rotating barotropic flow over finite isolate topography. Journal of Fluid Mechanics, 101, 281-306.

Buzzi, A., and A. Speranza (1979) Stationary flow of quasi-geostrophic, stratified atmosphere past finite amplitude obstacles. Tellus, 31, 1-12.

Fandry, C.B., R.L. Hughes and L.M. Leslie (1983) Stationary waves forced by topography in a vertically sheared, stratified, rotating fluid. Journal of the Autrailian Mathematical Society Series B 25, 127-144.

Flierl, G.R. (1978) Models of vertical structure and the calibration of two-layer models. Dynamics of the Atmosphere and Oceans, 2, 341-381.

Flierl, G.R. (1984) Rossby wave radiation from a strongly nonlinear warm eddy. Journal of Physical Oceanography, 14, 47-58.

Gould, J.W., R. Hendry, and H.E. Huppert (1981) An abyssal topographic experiment. Deep-Sea Research, 28A, 409-440.

Hide, R. (1961) Origin of Jupiter's Great Red Spot. Nature, 190, 895-896.

Hide, R., and A. Ibbetson (1966) An experimental study of Taylor columns. Icarus, 5, 279-290.

Hide, R., A. Ibbetson, and M.J. Lighthill (1968) On the slow transverse flow past obstacles in a rapidly rotating fluid. Journal of Fluid Mechanics, 32, 251.

Hogg, N.R. (1973) On the stratified Taylor column. Journal of Fluid Mechanics, 58, $517-537$.

Holloway, G., and P. Muller (1990) Topographic stress in the oceans. EOS, Transactions, American Geophysical Union, 71, 343-344.

Huppert, H.E. (1975) Some remarks on the initiation of inertial Taylor Columns. Journal of Fluid Mechanics, 67, 397-412.

Huppert, H.E. and Bryan, K. (1976) Topographically generated eddies. Deep-Sea Research, 23, 655-679.

Ingersoll, A.P. (1969) Inertial Taylor columns and Jupiter's Great Red Spot. Journal of Atmospheric Science, 26, 744-752. 
Jacobs, S.J. (1964) The Taylor column problem. Journal of Fluid Mechanics, 58, 517-537.

James, I.N. (1980) The forces due to geostrophic flows over shallow topography. Geophysical and Astrophysical Fluid Dynamics, 9, 159-177.

Janowitz, G.S. (1975) The effect of bottom topography on a stratified flow in the beta plane. Journal of Geophysical Research, 80, 4163-4168.

Johnson, E.R. (1977) Stratified Taylor columns on a beta-plane. Geophysical and Astrophysical Fluid Dynamics, 9, 159-177.

Johnson, E.R. (1978) Trapped vortices in rotating flow. Journal of Fluid Mechanics, 86, 209-224.

Johnson, E.R. (1979) Finite depth stratified flow over topography on a beta-plane. Geophysical and Astrophysical Fluid Dynamics, 12, 35-43.

Johnson, E.R. (1983) Taylor columns in a horizontally sheared flow. Geophysical and Astrophysical Fluid Dynamics, 24, 143-164.

Johnson, E.R. (1984) Starting flow for an obstacle moving transversely in a rapidly rotating fluid. Journal of Fluid Mechanics149, 71-88.

Kozlov, V.F. (1983) The Method of Contour Dynamics in Model Problems of the Ocean Topographysical Cylogenesis. Izvestiya, Atmospheric and Ocean Physics. 1983, 635-640.

Lorenz, E.N. (1972) Barotropic instability of Rossby wve motion. Journal of Atmospheric Science, 29, 258-264.

Meacham, S.P. (1988) Finite area beta-plane dipoles. Unpublished manuscript.

Meacham, S.P. (1990) Meander evolution on quasi-geostrophic jets. Journal of Physical Oceanography, submitted.

McCartney, M.S. (1975) Inertial Taylor columns on a beta plan. Journal of Fluid Mechanics, 68, 71-95.

McKee, J.W. (1971) Comments on a Rossby wake due to an island on an easward current, Journal of Physical Oceanography, 1, 187-.

Merkine, L. and E. Kalnay-Rivas, 1976: Rotating stratified flow over finite isolated topography. Journal of Atmospheric Science, 33, 908-921.

Miles, J.W., and H.E. Huppert (1968) Lee waves in a stratified flow. Part 2. Semicircular obstacle. Journal of Fluid Mechanics, 33, 803-814. 
Nof, D. (1988) The fusion of non-linear eddies. Journal of Physical Oceanography, 18, 887-905.

Owens, W.B. and N. G. Hogg (1980) Oceanic observations of stratified Taylor columns near a bump. Deep-Sea Research, 27A, 1029-1045.

Polvani, L.M. (1988) Geostrophic Vortex Dynamics, Ph.D. Thesis, MIT/WHOI.

Rhines, P.B. (1989) Deep Planetary Circulation and Topography: Simple Models of Midocean Flows. Journal of Physical Oceanography, 19, 1449-1470.

Rhines, P.B., and W.R. Young (1982) Homogenization of potential vorticity within closed gyres. Journal of Fluid Mechanics, 122, 347-367.

Richardson, P.L. (1980) Anticyclonic eddies generated near the Corner Rise seamounts. Journal of Marine Research, 38, 673-686.

Roden, G.I., and B.A. Taft (185) Oceanographic aspects of the Emperor seamounts region. Journal of Geophysical Research, 90, 839-855.

Roden, G.I. (1987) Effect of seamounts and seamount chains on ocean circulation and thermohaline structure. In: Seamounts, Islands and Atolls, B. Keating, P. Fryaer, R. Batiza, and G. Boehlert, eds., Geophyscial Monograph 43, American Geophysical Union, Washington, D.C., 335-354.

Schar, C., and H.C. Davies (1988) Quasi-geostrophic stratified flow over isolated finite amplitude topography. Dynamics of the Atmosphere and Oceans, 11, 237-306.

Straub, D.N. and P.B. Rhines (1990) Effects of large-scale topography on abyssal circulation. Journal of Marine Research, 48, 223-253.

Taylor, G.I. (1917) Motion of solids in fluids when the flow is not irrotational. Proceedings of the Royal Socciety A, 93, 99-113.

Taylor, G.I. (1923) Experiments on the motion of solid bodies in rotating fluids. Proceeding of the Royal Society A, 104, 213-218.

Treguier, A.M. and J.C. McWilliams (1990) Topographic influences on wind driven stratified flow in a beta-plane channel: an idealized model for the Antarctic Circumpolar Current. Journal of Physical Oceanography, 20, 321-343.

Uchupi, E.J., J.D. Phillips and K.E. Prada (1970) Origin and structure of the New England Seamount Chain. Deep-Sea Research, 17, 483-495. 
Vastano, A. C. and B.A. Warren (1976) Perturbations to the Gulf Stream by Atlantis II Seamount. Deep-Sea Research, 23, 681-694.

Vaziri, D. and D. L. Boyer (1971) Rotating flow over shallow topographyies. Journal of Fluid Mechanics, 50, 79-95.

Verron, J. (1986) Topographic eddies in temporally varying oceanic flows. Geophysical and Astrophysical Fluid Dynamics, 35, 257-276.

Verron, J. and C. Le Provost (1985) A numerical study of quasigeostrophic flow over isolated topography. Journal of Fluid Mechanics, 154, 231-252

White, W.B. (1971) A Rossby wake due to an island in an eastward current. Journal of Physical Oceanography, 161-168.

Zubusky, N.J., M.H. Hughes, and K.V. Roberts (1979) Contour dynamics for th Euler equations in two dimensions. Journal of Computational Physics, 30, 96106. 


\section{APPENDIX A}

In order to solve the integral equation 2.11 numerically, it must be discretized. Each linear equation is a discretization of the integral equation evaluated at $\mathbf{x}_{i}^{\prime}$. The contributions from the first integral of 2.11 is

$$
I_{i}=\sum_{j=0}^{N} I_{i j}=\sum_{j=0}^{N} \int_{\mathbf{x}_{j}}^{\mathbf{x}_{j+1}} \phi\left(\mathbf{x}^{\prime \prime}\right) \frac{\partial G}{\partial n^{\prime}}\left(\mathbf{x}_{i}, \mathbf{x}^{\prime}\right) d s^{\prime}
$$

and $I_{i j}$ for $j \neq i-1, i$ can be estimated as

$$
I_{i j}=\frac{1}{2}\left[\phi\left(\mathbf{x}_{j}\right)+\phi\left(\mathbf{x}_{j+1}\right)\right], \frac{\partial G}{\partial n^{\prime}}\left(\mathbf{x}_{i}, \mathbf{x}_{j+1 / 2}\right)\left|\mathbf{x}_{j+1}-\mathbf{x}_{j}\right|,
$$

where $\mathbf{x}_{j+1 / 2}=\frac{1}{2}\left[\mathbf{x}_{j}+\mathbf{x}_{j+1}\right]$ and the normal direction is taken perpendicular to $\mathbf{x}_{j+1}-\mathbf{x}_{j}$. Near the singularity of $G$, when $j=i$ or $i+1, I_{i j}$ is evaluated by allowing the contour between $\mathbf{x}_{i}$ and $\mathbf{x}_{i+1}$ to be a circular arc with radius of curvature $r_{c}$. Along this portion of the arc, the singular part of the Green's function can be approximated by its most singular part; that is $\partial G / \partial n^{\prime}\left(\mathbf{x}_{i}, \mathbf{x}^{\prime}\right)=1 / 4 \pi r_{c}$ for any value of $b$. Then $\phi$ is expanded in a Taylor series along the arc

$$
\phi(\theta)=\phi_{j}+\frac{\phi_{j}-\phi_{j-1}}{\theta_{j}-\theta_{j-1}}\left(\theta_{j}-\theta\right)
$$

for $\theta_{j-1}<\theta<\theta_{j}$ and

$$
\phi(\theta)=\phi_{j}+\frac{\phi_{j+1}-\phi_{j}}{\theta j+1-\theta_{j}}\left(\theta-\theta_{j}\right)
$$

for $\theta_{j-1}<\theta<\theta_{j}$, where $x$ is the point on the arc of the circle with radius $r_{c}$ and angle $\theta$. Then we change variables to $\theta$ in A.1 and integrate with respect to $\theta$ to find

$$
I_{i, i}=\frac{1}{4 \pi}\left(\theta_{j+1}-\theta_{j}\right) \frac{1}{2}\left(\phi_{j}+\phi_{j+1}\right)
$$

and

$$
I_{i, i-1}=\frac{1}{4 \pi}\left(\theta_{j}-\theta_{j-1}\right) \frac{1}{2}\left(\phi_{j}+\phi_{j-1}\right) .
$$

Now $\theta_{j}, \theta_{j-1}$ and $\theta_{j+1}$ depend on $x_{j}, x_{j-1}$, and $x_{j+1}$. Thus in general we can write

$$
I_{i}=\sum_{j=0}^{N}\left(a_{j}\left(\mathbf{x}_{i}\right)+b_{j-1}\left(\mathbf{x}_{i}\right)\right) \phi\left(\underline{x_{j}}\right)=\sum_{j=0}^{N} l_{i j} \phi\left(\mathbf{x}_{j}\right) .
$$

The contribution from the next integral of 2.11 away from the singularity can be performed similarly to the first integral,

$$
M_{i}=\sum_{j=0}^{N} M_{i j}=\sum_{j=0}^{N} \int_{\mathbf{x}_{j}}^{\mathbf{x}_{j+1}} G\left(\mathbf{x}^{\prime}, \mathbf{x}\right) \frac{\partial \phi}{\partial n^{\prime}}\left(\mathbf{x}_{i}, \mathbf{x}^{\prime}\right) d s^{\prime} .
$$


Then we estimate $M_{i j}$ for $j \neq i-1, i$ by

$$
M_{i j}=\frac{1}{2}\left[\frac{\partial \phi\left(\mathbf{x}_{j}\right)}{\partial n}+\frac{\partial \phi\left(\mathbf{x}_{j+1}\right.}{\partial n}\right] G\left(\mathbf{x}_{i}, \mathbf{x}_{j+1 / 2}\right)\left|\mathbf{x}_{j+1}-\mathbf{x}_{j}\right| \text {. }
$$

Near the singularity, we expand $\frac{\partial \phi}{\partial n}$ in a Taylor series

$$
\frac{\partial \phi}{\partial n}=\frac{\partial \phi}{\partial n}+\frac{\frac{\partial \phi}{\partial n}{ }_{j}-\frac{\partial \phi}{\partial n}{ }_{j-1}}{s}
$$

where $s=\left|\mathbf{x}_{j}-\mathbf{x}_{j-1}\right|$. Expanding the Green's function about $s=0$ we find that to lowest order

$$
G(s)=\frac{1}{2 \pi}\left(\ln |s|+\ln b^{\frac{1}{2}}\right) .
$$

We then integrate A.3 with respect to $s$ between $-s\left(\mathbf{x}_{j}\right)$ and 0 . To find the $M_{i, i}$ we just replace $j+1$ for $j-1$. We can thus write

$$
l_{i, j} \phi_{j}-m_{i, j} \phi_{j}^{(a)}=\delta_{i j} \phi_{j}
$$

This set of equations is inverted using an IMSL routine. In order to improve the guess for the boundary, a relaxation scheme is used, again following Meacham (1988). The deviation of $\phi+\psi^{(a)}$ from $C$ is defined as $e_{j}$ at each point, and the new positions for the points on the contour are given by

$$
\left(x_{i}, y_{i}\right)=\left(x_{i}, y_{i}\right)-\alpha \frac{e_{i}}{\partial \phi / \partial n}\left[\frac{d y}{d s}\left(\mathbf{x}_{i}\right),-\frac{d x}{d s}\left(\mathbf{x}_{i}\right)\right] .
$$

for each $i$, and the direction is given by an approximation to the tangent direction at $x_{i}$. Typically, about 30 points are used and $\alpha$ is 0.1 or 0.2 . The procedure works best when the initial guess for $\partial D$ is contained within the correct solution for a given value of $C$. 


\section{APPENDIX B}

The shallow water model that we use in Chapter 3 is valid as long as the aspect ratio of the fluid is small. That is

$$
H^{2} / \mathcal{L}^{2}<<1,
$$

where $H$ is the depth of the fluid and $\mathcal{L}$ is the horizontal length scale in the problem. When there is a finite step in the topography, the validity of this requirement is questionable because length scale of the variations in the topography has gone to zero $(\mathcal{L}$ is small) and the aspect ratio becomes large. To understand if we are justified in using the shallow water model in the limit of a finite step in the topography, we consider the flow field induced when a uniform flow impinges on topography which changes from one value to another linearly over a finite distance and then take the limit of this distance going to zero. In this limit, we will show that the matching conditions used in Chapter 3 are correct. We use the barotropic shallow water equations given in Section 3.3.1 and use the scaling developed in that section.

The depth of the fluid for this problem is given by

$$
h= \begin{cases}1 & x<0 \\ 1-\frac{h_{0}}{\alpha} x & 0<x<\alpha \\ 1-h_{0} & x>\alpha .\end{cases}
$$

Upstream of the topography, the flow is zonal and uniform with size 1 . There are no $y$ variations and the continuity equation gives

$$
h u=1 .
$$

To find $v$ we use the potential vorticity equation

$$
v_{x}=\frac{h-1}{\epsilon} .
$$

We integrate this equation with respect to $x$ and find that

$$
v= \begin{cases}0 & x<0 \\ -h_{0} x^{2} / 2 \alpha \epsilon & 0<x<\alpha \\ -h_{0}(x-\alpha / 2) / \epsilon & x>\alpha .\end{cases}
$$

If we now take the limit $\alpha \rightarrow 0$ of B.1 we find that $v$ and $u h$ are continuous at $x=0$, the edge of the topography. We now evaluate the pressure. The Bernoulli function 
is conserved on streamlines and for this problem is given by $B=\psi$ where $\psi$ is the transport stream function. We can find $P$ from this,

$$
P=\psi-\frac{\epsilon}{2}\left(u^{2}+v^{2}\right) .
$$

For continuous topography ( $\alpha$ finite), the pressure is continuous in $x$. When we take the limit $\alpha \rightarrow 0$ we find that the pressure is discontinuous at $x=0$ and the jump in the pressure is

$$
P\left(x=0^{+}\right)-P\left(x=0^{-}\right)=\frac{\epsilon}{2} \frac{h_{0}\left(2-h_{0}\right)}{\left(1-h_{0}\right)^{2}} .
$$

Only in the quasi-geostrophic limit $(\epsilon \rightarrow 0)$ is the pressure continuous.

We have come to the conclusion that for the model that we use in Section 3.3 , the pressure is discontinuous on the edge of the topography. To reconcile this with the use of the shallow water equations, we require that $H<<\alpha L<<L$ so that the aspect ratio of the fluid is small, but the change in the topography can be approximated as a step. This example justifies the matching conditions that we apply in Section 3.3: that the radial transport and the tangential velocity are continuous. 


\section{APPENDIX C}

The same method that is used in Chapter 4 can be used to study the interaction of isolated eddies with a cylindrical obstacle that reaches to the surface. This physical situation was used initially to test the method. It can be done in both one and two layers.

The evolution of a patch of constant vorticity near a circular obstacles is considered. In general the stream function $\psi$ satisfies

$$
\partial_{t} \Pi+J(\psi, \Pi)=0,
$$

where $\Pi=\nabla^{2} \psi$ and $\psi$ satisfied boundary condition on the circular cylinder $(r=1)$. There is no flow into the boundary

$$
u \cdot \hat{n}=0,
$$

and there is no circulation about the island

$$
\oint u \cdot \hat{t} d l=0 .
$$

In one layer, one might think initially that the appropriate Green's function could be found such that the boundary conditions are automatically satisfied. The Green's function can be found for the problem of flow of a fluid due to an arbitrary patch of vorticity near a circular cylinder that has no flow into it as well as no circulation around it. This Green's function is

$$
G\left(x, x^{\prime}\right)=\frac{1}{2 \pi} \ln \frac{\left|\mathbf{x}-\mathbf{x}^{\prime}\right|}{\left|\mathbf{x}^{\prime}\right|\left|\frac{\mathbf{x}^{\prime}}{\left|\mathbf{x}^{\prime}\right|^{2}}-\mathbf{x}\right|},
$$

and it is found by the method of images. In order to use the method of contour dynamics, the area integral that determines the stream function must be transformed into a contour integral to be able to find the velocity. This requires that

$$
\frac{\partial G}{\partial x}= \pm \frac{\partial G}{\partial x^{\prime}},
$$

and the same for the derivatives in $y$. The Green's function does not contain this symmetry. This can be understood by considering the analogous problem of a patch of vorticity near a straight wall where the Green's function can be found by the method of images. One can then conformal map the problem to a flow around a circle. However, the conformal mapping does not transform the boundary conditions which are applied only in $x$ and $y$ coordinates and not in the $x^{\prime}$ and $y^{\prime}$ coordinates, and the transformation changes the symmetry of the Green's function. 
Insteady the solution is found by letting the solution be a sum of the solution when there is no boundary and a zero vorticity solution,

$$
\psi=\frac{1}{2 \pi} \iint \Pi \ln \left|\mathbf{x}-\mathbf{x}^{\prime}\right|+\sum_{n=-N / 2}^{n=N / 2} a_{n} r^{-n} e^{-i n \theta}+b \ln r .
$$

The contribution to the velocity from the vortex patch can be found in the usual way by performing a contour integral,

$$
\left(u^{p}, v^{p}\right)=-\frac{1}{2 \pi} \oint_{\partial D} \ln \left|x-x^{\prime}\right|\left[d x^{\prime}, d y^{\prime}\right] .
$$

In cylindrical coordinates the radial velocity $\frac{1}{r} \psi_{\theta}$ and the azimuthal velocity $\psi_{\mathrm{r}}$ are given by

$$
-\frac{1}{r} \psi_{\theta}=u^{p} \cos \theta+v^{p} \sin \theta+\sum_{n=-N / 2}^{n=N / 2} i n a_{n} r^{-|n|} e^{-i n \theta}
$$

and

$$
\psi_{r}=-u^{p} \sin \theta+v^{p} \cos \theta+\sum_{n=-N / 2}^{n=N / 2}-|n| a_{n} r^{-|n|} e^{-i n \theta}+\frac{b}{r} .
$$

The particular solution can be evaluated at $2 N$ points around the circle at $r=1$ an expanded in a fourier series in $\theta$ using an FFT

$$
u^{p} \cos \theta+v^{p} \sin \theta=\sum_{n=-N / 2}^{n=N / 2} \hat{u}_{n} e^{-i n \theta} .
$$

Thus applying the boundary condition C.1 gives

$$
a_{n}=-\frac{\hat{u}_{n}}{i n} .
$$

Likewise, applying C.2 gives

$$
b=\frac{1}{2 \pi} \int_{0}^{2 \pi}\left(-u^{p} \sin \theta+v^{p} \cos \theta\right) d \theta .
$$

Finally, to step forward the contour, we evaluate the contribution to the velocity from the zero vorticity solution and from the contour integral at the points on the contour.

In the example shown in Figures C.1 and C.2, a background flow with zero vorticity is added

$$
\psi_{\text {background }}=-r \sin \theta+q \frac{\sin \theta}{r}
$$


and the vortex patch has vorticity $q$. The vortex patch does not at any time intersect the boundary and the area is conserved to within a few tenths of a percent using $N=32$.

The accuracy of the location of the contours depends on the time-step, the spacing between the points on the contour and $N$. The trade off between the point spacing and $N$ can be quantified. For equal accuracy from both the spatial contributions we should require that

$$
\Delta x^{2}=R^{-N / 2} \text {. }
$$

where $R$ is a representative distance from the cylinder. Then we can solve for $N$ when the number of points on the contour is 50 .

$$
\frac{4 \ln \frac{2 \pi}{50}}{\ln R}=N
$$

When $R$ is order one, then $N=8$ should be adequate. In all of the runs in Chapter $4, N=32$ so that the error associated with truncating the sum is at least as good as the error associated in the point spacing. 

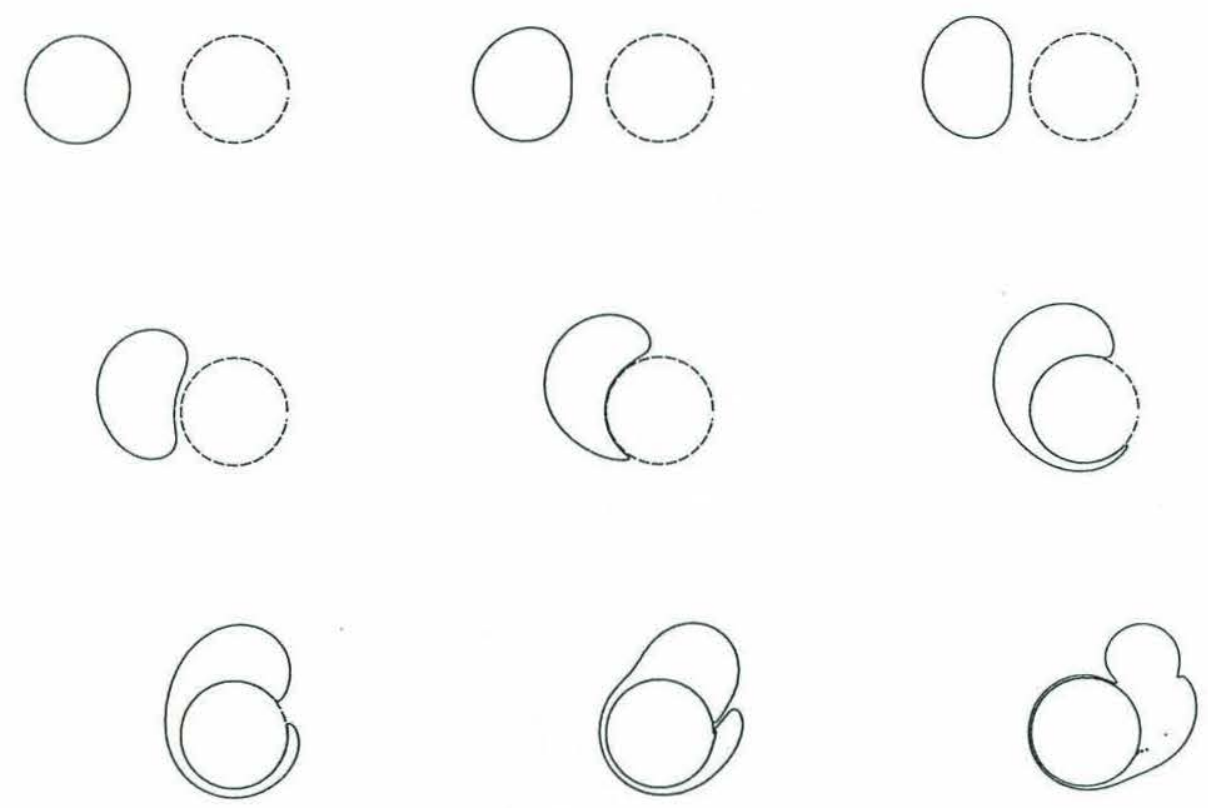

Figure C.1: Evolution of the contours when $q=5$ at $t=0,0.5, \ldots, 4$. The location of the contour looks quite similar to what Nof (1988) found in a laboratory experiment in which he generated an eddy near a cylinder by continuously injecting fluid to form the eddy. When the eddy became large enough, it began to interact with the cylinder in a very similar manor as that shown here. 

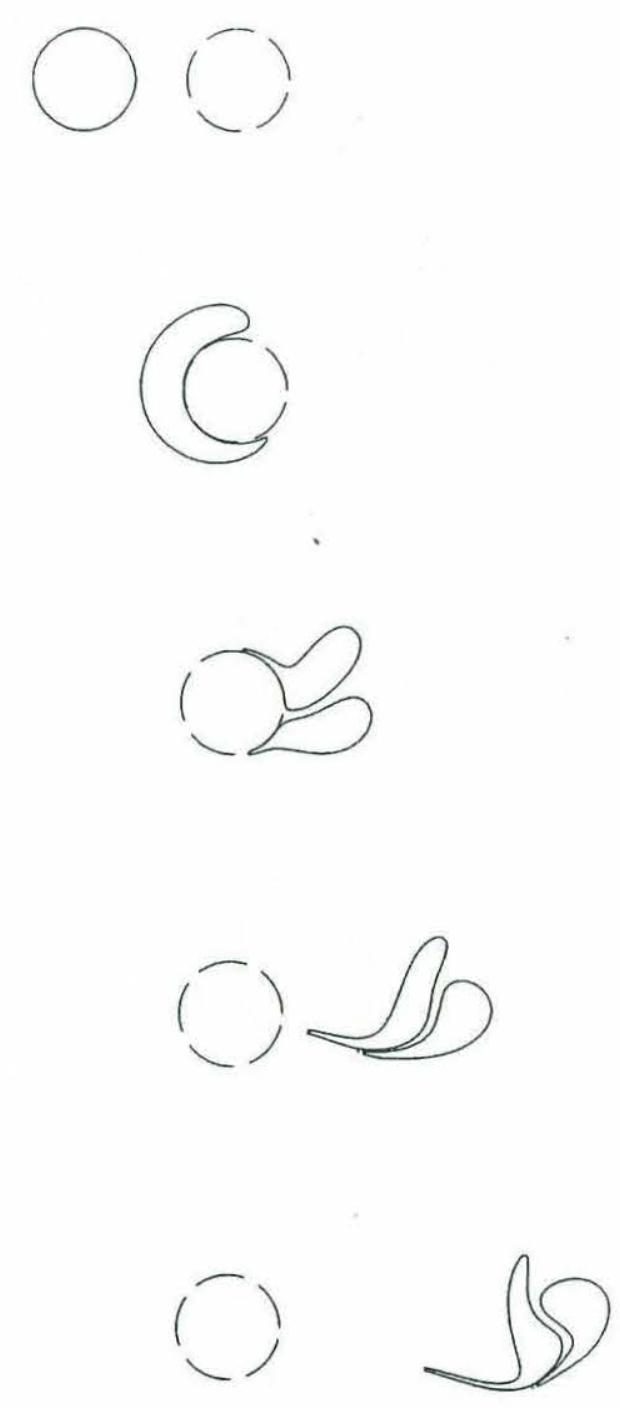

Figure C.2: Evolution of the contours when $q=1$ at $t=0,1, \ldots, 4$. The eddy is split by the cylinder and then merges after the two pieces go around the cylinder. 